\title{
SISTEMAS MODAIS DE CONHECIMENTO
}

\author{
Flavio Shigeo Yamamoto
}

DISSERTAÇÃO APRESENTADA

$\mathrm{AO}$

INSTITUTO DE MATEMÁTICA E ESTATÍSTICA

DA

UNIVERSIDADE DE SÃO PAULO

PARA

OBTENÇÃO DO GRAU

DE

MESTRE EM CIÊNCIA DA COMPUTAÇÃO

Área de Concentração: Lógica e Sistemas de Conhecimento Orientador: Prof. Dr. Ricardo Bianconi

- São Paulo, 11 de maio de 2003 - 


\section{SISTEMAS MODAIS DE CONHECIMENTO}

Flavio Shigeo Yamamoto

Este exemplar corresponde à redação final da dissertação apresentada por Flavio Shigeo Yamamoto, devidamente corrigida e aprovada pela Comissão Julgadora

São Paulo, 11 de maio de 2003

\section{Comissão Julgadora}

- Prof. Dr. Ricardo Bianconi - IME-USP

- Prof. Dr. Marcelo Finger - IME-USP

- Prof. Dr. Marcelo Esteban Coniglio - IME-USP 
$\grave{A}$ minha mãe

e à memória de meu pai com afeto e estima.

Za Gocu sa privrženošću i ljubavlju. 


\section{Agradecimentos}

Ao orientador Ricardo Bianconi, pela paciência que teve durante todo o longo período que durou este trabalho.

À Gordana, pelo amor, carinho, compreensão e incentivo durante a elaboração deste texto.

À Erica, pelo afeto e dedicação durante os momentos pelos quais passamos.

À dupla-dinâmica Scal e Sonti, que me vem "atrapalhando" desde a graduação, mas por diversas vezes indicaram erros e deficiências deste texto. Aos companheiros de graduação Jõao Paulo, Clóvis e Jefferson pelo convivio e piadas. À Gleice, Ivana, Lúcia Ikemoto, Luciano Silva, Luiz "Mogi", Rolo, Samuel, aos amigos do ime e à B. Ilić (pelo livro do van Benthem).

Aos Profs. Elias Roma Neto e Luiz Gonzaga Xavier de Barros pelo apoio, em especial ao Prof. Jair Minoro Abe pelas conversas e estímulo. Aos amigos Marcos e Claudio.

Ao pessoal do café, Dalvina, Jovita e Maria, a quem devo meu "vício" pelo bem-humorado-café servido naquele balcão.

Aos amigos da biblioteca, Cristina, Lúcia, Márcia, Max, Renato, Sílvia, Vera, por terem me suportado ao longo desses anos e às amigas Célia e Marina pelas conversas bem-humoradas e por me considerarem um "quaseauxiliar" da biblioteca.

A todos que me auxiliaram direta ou indiretamente em minha formação neste instituto. 


\section{Resumo}

Investigamos características e propriedades de sistemas multi-agentes baseados em conhecimento. Estudamos axiomatizações corretas e completas que capturam e manipulam as noções de conhecimento, conhecimento comum e conhecimento distribuído. Complementamos o estudo dos sistemas de conhecimento caracterizando-os de acordo com a classe de complexidade computacional a que pertencem: fazemos isso expondo as dificuldades existentes nos procedimentos de decisão em termos da complexidade computacional das diversas axiomáticas apresentadas. Verificamos que enquanto o problema de decisão da satisfatibilidade de uma fórmula em S5 com um agente é $N P$-completo, o problema para vários agentes é PSPACE-completo e que a adição do operador de conhecimento comum à linguagem altera o problema para EXPTIME.

Palavras-Chaves: Conhecimento, conhecimento comum, conhecimento distribuído, decidibilidade, satisfatibilidade, tableaux, complexidade. 


\begin{abstract}
We investigate features and properties of knowledge-based multi-agent systems. We study correct and complete axiomatizations which capture and handle the notions of knowledge, common knowledge and distributed knowledge. We extend the study of knowledge systems classifying them according to the computational complexity class to which they belong: we do that exposing the existing difficulties in the decision procedures in terms of the computational complexity of the several axiomatizations presented. We verify that while the problem of deciding satisfiability of a formula in S5 with one agent is $N P$-complete, the problem for many agents is PSPACE-complete and the addition of the common knowledge operator to the language turns the problem to EXPTIME.
\end{abstract}

Palavras-Chaves: Knowledge, common knowledge, distributed knowledge, decidability, satisfability, tableaux, computational complexity. 


\section{Sistemas Modais \\ de Conhecimento}




\section{Índice}

Introdução $\quad$ i

Motivação e Primeiras Noções . . . . . . . . . . . . . . . i

Apresentação ..................... iii

1 Preliminares 1

1.1 Ingredientes Históricos . . . . . . . . . . . . 2

1.2 Verdade, Conhecimento e Mundo Possível . . . . . . . . 4

1.3 O Cálculo Proposicional Clássico CP . . . . . . . 7

1.4 As Noções de Verdade em CP . . . . . . . . . . . . 10

$2 \mathrm{O}$ sistema $\mathrm{K}_{n} \quad 13$

2.1 o Sistema $K_{n} \ldots \ldots \ldots \ldots \ldots \ldots$

2.2 Semântica para $K_{n}$ : satisfatibilidade e validade . . . . . 18

2.3 Inferência, Correção e Completude em $\mathrm{K}_{n} \ldots \ldots . . . . .24$

3 Extensões do Sistema $K_{n} \quad 29$

3.1 Os sistemas: $\mathrm{T}_{n}, \mathrm{~S}_{n}, \mathrm{~S} 5_{n}$ e $\mathrm{KD} 5_{n} \ldots \ldots \ldots \ldots 29$

3.2 Correção e Completude . . . . . . . . . . . . 35 
3.3 Modelos, Fechos e Frames . . . . . . . . . . . . . 36

3.4 Estruturas Finitas . . . . . . . . . . . . . . 42

4 Conhecimento Comum e Conhecimento Distribuído 47

4.1 Conhecimento Comum . . . . . . . . . . . . . . 48

4.2 Conhecimento Distribuído . . . . . . . . . . 58

5 Decidibilidade e Complexidade $\quad 73$

5.1 Decidibilidade. . . . . . . . . . . . . . . . 74

5.2 Preliminares.................... 78

5.2.1 Máquinas de Turing e Complexidade Computacional . 78

5.2 .2 Classes de Complexidade . . . . . . . . . 79

5.2.3 Fórmulas Booleanas Quantificadas ......... 81

5.3 A classe de complexidade de S5 e KD45 . . . . . . . 83

5.4 Limitantes inferiores . . . . . . . . . . . 85

5.5 Procedimentos de decisão usando tableau . . . . . . . . . 93

5.6 Decisão envolvendo conhecimento comum . . . . . . . 111

Referências Bibliográficas $\quad 118$

$\begin{array}{ll}\text { Índice Remissivo } & 126\end{array}$ 


\section{Introdução}

Neste trabalho tratamos da lógica modal proposicional aplicada à construção de sistemas multi-agentes baseados em conhecimento. Analisamos os sistemas modais $\mathrm{K}_{n}, \mathrm{~T}_{n}, \mathrm{~S} 4_{n}, \mathrm{~S} 5_{n}$, e $\mathrm{KD} 45_{n}$ quanto à correção e completude em relação à semântica atribuída a cada um dos sistemas. Estudamos a complexidade computacional desses sistemas em relação ao problema de decidibilidade. Todo o conteúdo deste trabalho, exceto pelo primeiro capítulo, é baseado ${ }^{1}$ no artigo de Halpern e Moses [36].

\section{Motivação e Primeiras Noções}

Em ciência da computação encontramos diversas aplicações da linguagem modal, por exemplo, em programação (Pratt [67], [68] e [69]), em programação concorrente (Henessy [39], [40], Milner [59] e Park [65]), no tratamento formal de protocolos em sistemas distribuídos (Fischer [28], Fagin [26] e Halpern [35]), nos métodos de especificação e verificação formal (Massaci [55]) em bancos de dados por meio da lógica temporal (Emerson [21]), no estudo de lingüística computacional (Morril [62, sec. 2.2], Carpenter [7] e van Benthem [85]), no estudo da computação quântica através da lógica quântica (Chiara [12]) e em construções de sistemas baseados em conhecimento (Fagin, Halpern, Vardi e Moses [24]). Essa variedade de aplicações se deve, em parte, à expressividade da linguagem modal.

\footnotetext{
${ }^{1}$ Dentre os textos que utilizamos, sobre o estudo e aplicaçōes da lógica modal, destacamos as obras de von Wright [87], Hintikka [41], van Benthem ([84] e [83]) e Fagin et all. [24]. Entre as mais recentes, citamos Girle [32], Blackburn [2], Carnielli [6] e Costa [16].
} 
No estudo sobre conhecimento as análises, em geral, tendem a considerar o conhecer de único agente, porém num diálogo, numa transação comercial ou no tratamento de protocolos (em sistemas distribuídos) o interessante é a interação do conhecimento entre os agentes. Os conceitos de conhecimento comum e conhecimento distribuído mostram-se úteis para analisar tais interações, por exemplo, a noção de conhecimento comum aparece como um pré-requisito para alcançar um entendimento entre agentes, fator crucial para o estudo dessas interações.

As linguagens das lógicas não clássicas são freqüentemente representadas por modificações ou extensões da linguagem clássica, pela remoção de alguma propriedade ou adição de novos operadores. A lógica modal enquadra-se nesse último caso. Ela é uma extensão da lógica proposicional clássica, pelo menos em princípio, com a inclusão de operadores modais.

Os operadores modais expressam a modalidade gramatical de uma sentença (Mints [60, pág. iv]), isto é, qualificam como verdadeira ou falsa uma proposição de acordo com o que distinguem: necessidade ${ }^{2}$, possibilidade, conhecimento, crença, obrigação ou propriedades temporais.

Tais operadores não são funções de valoração como os conectivos lógicos do cálculo proposicional clássico. Isto pois, em geral, não se pode determinar a semântica de uma expressão da forma $\square \varphi$ ou $\diamond \varphi$, unicamente a partir da semântica atribuída a $\varphi$. São exceções: se $\varphi$ é verdadeira, então $\diamond \varphi$ também é verdadeira, e se $\varphi$ é falsa, então $\square \varphi$ também é. Logo, os operadores modais não podem ser caracterizados a partir dos conectivos clássicos e sua semântica difere da do cálculo proposicional clássico.

Em geral, adota-se a semântica dos mundos possíveis (ou semântica de Kripke) para as diversas aplicações da lógica modal proposicional, por ser mais intuitiva e oferecer recursos tais que qualquer mudança na axiomática pode ser capturada pelas diferentes interpretações atribuídas a esta semântica, mais precisamente, pelos diferentes significados atribuídos ao termo mundo possivel.

Por exemplo, em PDL ( Program Dinamic Logic-Pratt [69]) um mundo possível pode ser interpretado como sendo o estado de um programa, já na lógica modal temporal um mundo possível pode ser considerado um ponto no tempo (cf. Fagin ([25, pág. 2] e Rijke [71, pág. 2]). No estudo dos sistemas de conhecimento, qual o papel dos mundos possíveis para capturar a noção intuitiva de conhecimento? Este assunto será abordado nas seções 1.2 e 1.4).

\footnotetext{
${ }^{2}$ A lógica modal costuma ser caracterizada como a lógica da necessidade e possibilidade por serem estas as modalidades mais investigadas, os símbolos usuais para representar tais modalidades são $\square$ e $\diamond$, respectivamente. Sintaticamente, $\diamond$ é definida a partir de $\square$ pelo uso da negação ( $\diamond$ equivale a $\neg \square \neg$ ), porém diferem muito quanto à semântica.
} 


\section{Apresentação}

Nosso estudo enfatiza os seguintes pontos:

- axiomatizações aplicadas a modelos de conhecimento,

- noções de conhecimento comum e conhecimento distribuído,

- procedimentos de decisão,

- a complexidade computacional dos procedimentos de decisão.

Para melhor situar o leitor sobre certos aspectos contidos no texto iniciamos o trabalho com um capítulo contendo um resumo da história da lógica modal ( $c f$. Blackburn [2] e Bull e Segerberg [5, págs. 1-16]).

Prosseguimos fazendo um brevíssimo estudo dos termos verdade, conhecimento e mundo possível, elementos que compõem a semântica para os sistemas de conhecimento. Estudamos especificamente (do ponto de vista conceitual) a relação intuitiva entre os termos mundo possivel e conhecimento.

Sobre os conceitos de verdade e conhecimento estudamos essencialmente os textos de Hintikka ([44] e [43]), Dunn [20], Kirkham [47] e Lehrer [50]. Sobre a noção de conhecimento destacamos von Wright [87], da Costa [15], Gettier [31], Lenzen [51], Levesque [53] e Tselishchev [82]. Delineamos o estudo desses dois conceitos baseados em Fagin [25] e Zalta [89].

No capítulo 2 estabelecemos uma linguagem que trata formalmente da noção de conhecimento, conforme Halpern e Moses [36]. A sintaxe e a semântica do sistema $\mathrm{K}_{n}$ segue conforme Chellas [11], Halpern [36] e Zalta [90]. Os resultados $K_{n}$ seguem de Fagin ([22] e [24]) e Halpern [36]. No capítulo seguinte, apresentamos extensões de $\mathrm{K}_{n}: \mathrm{T}_{n}, \mathrm{~S} 4_{n}, \mathrm{~S} 5_{n}$, e $\mathrm{KD} 45_{n}$ seguido dos respectivos resultados de correção e completude (Chellas [11], Halpern [36] e Zalta [90]). Investigamos propriedades de algumas estruturas e frames. Finalizamos exibindo os resultados de correção e completude sobre estruturas de Kripke finitas (Fagin ([22] e [24]) e Halpern [36]).

No capítulo 4 analisamos extensões com a inclusão dos operadores conhecimento comum e conhecimento distribuído (Milgrom [58] e Halpern [35]). Mostramos que mesmo com a inclusão desses operadores podemos obter axiomatizações completas (Halpern e Moses [36]).

No quinto capítulo estudamos questões relacionadas ao problema da decidibilidade e validade de uma fórmula em um sistema modal (Levesque [53] e Halpern [36]). Investigamos a complexidade computacional dos sistemas de conhecimento em relação ao problema da satisfatibilidade (Garey [30], del Cerro [8], Stockmeyer e Meyer [76], Ladner [48], Halpern [36] e Micali [57]). 


\section{Preliminares}

Estamos interessados em manipular expressões da forma:

$i$ sabe $\varphi, \quad i$ sabe que $\varphi, \quad i$ conhece $\varphi$ ou $i$ conhece que $\varphi^{1}$.

Especificamente, as expressões que nos dizem respeito são aquelas em que $i$ pode ser substituída por um agente ${ }^{2}$ (pelo nome de uma pessoa) e $\varphi$ por uma proposição ${ }^{3}$ (por uma sentença declarativa). Para manipularmos tais expressões construímos os sistemas multi-agentes baseados em conhecimento.

Nossa abordagem, para a construção desses sistemas, é pelo método axiomático ${ }^{4}$. Antes de partirmos para a construção, propriamente dita, tecemos comentários sobre cada um dos elementos e de como eles compõem o cenário em que desenvolvemos este trabalho. Não é objetivo nosso fazer uma análise aprofundada sobre os aspectos filosóficos dos termos em questão, mas

\footnotetext{
${ }^{1}$ A última forma pode, talvez, ser alterada para: $i$ tem conhecimento de que $\varphi$. Questões como: os casos não apropriados para o uso do sabe, diferenças entre os termos sabe e sabe que, conhece e conhece que e a preferência por um ou outro não são tópicos deste estudo. Uma discussão mais detalhada pode ser encontrada em Hegenberg $[38$, cap. 8, 9].

${ }^{2}$ Os agentes que figuram na semântica dos mundos possíveis são epistêmicos, cada um deles reúne um conjunto ideal de propriedades da razão idêntico a todos. Para maiores detalhes consulte Duc [19] e Wooldridge [88].

${ }^{3}$ Vide seção 1.3

${ }^{4}$ Por este processo a natureza dos objetos envolvidos não tem relevância para análise dos resultados desejados, sendo necessários somente os postulados que caracterizam os objetos envolvidos. A manipulação puramente algébrica "deixa de lado" a intuição, a preocupação em compreender os fundamentos fica em segundo plano. Isto não impede o desenvolvimento do estudo dos sistemas lógicos que, em geral, independe de soluçōes das dificuldades filosóficas envolvidas.
} 
dentro de certos limites obter características que nos possibilitem manuseálos sob regras bem definidas.

Na seção 1.1 fazemos uma brevíssimo apresentação da história da lógica modal. Destacamos três das diversas correntes que compõe a moderna lógica modal (cf. Blackburn [2] e Bull e Segerberg [5, págs. 1-16]).

$\mathrm{Na}$ seção 1.2 expomos sob qual teoria da verdade abordamos os sistemas de conhecimento, lembrando que desejamos encontrar uma semântica que preserve a noção de verdade adequada (que possamos correlacionar) ao que entendemos por conhecimento.

Nas seções 1.3 e 1.4 analisamos as noções de verdade na lógica clássica e comentamos brevemente suas relações com as regras de inferência.

\subsection{Ingredientes Históricos}

Duas das dificuldades em analisar o quanto o estudo da lógica modal evoluiu desde seus primeiros estudos até os dias de hoje estão na grande quantidade de trabalhos publicados (principalmente em época recente) e na diversidade de enfoques, que vão desde o uso para tratar questões de caráter filosófico ${ }^{5}$ (Girle [32]), no estudo da Lógica da Prova ${ }^{6}$ (Boolos [3]), em questões envolvendo a gramática de Montague (Montague [61] e van Benthem [85]), aplicações em economia (Milgrom [58]), etc.

No entanto, podemos descrever a situação da moderna lógica modal em seus diversos períodos classificando a de acordo com a perspectiva dada a semântica, conforme Bull e Segerberg [5] e Blackburn [2].

Apesar do estudo das modalidades datar dos tempos de Aristóteles, em tratados encontrados no Organon e de ter sido objeto de estudo dos estóicos e megáricos, foi somente em período recente que a lógica modal se desenvolveu. Não há uma data que marque o início da moderna lógica modal, por exemplo, em Bull e Segerberg [5] e van Benthem [85] encontramos como início o ano de 1912 com a publicação de Implication and the algebra of logic ${ }^{7}$ em Mind por C. I. Lewis. Já Blackburn [2, sec. 1.7] e outros, citam 1906 ano da publicação de Symbolic logic and its Applications de Hugh MacColl.

\footnotetext{
${ }^{5}$ Levando a análise de tais problemas a um novo nível de discussão, o formal. Por exemplo, os argumentos podem ser vistos como modalidades (Girle [32, cap. 1]).

${ }^{6}$ The Provability of Logic em Boolos [3].

${ }^{7} \mathrm{~A}$ motivação original de Lewis não era investigar as noções de necessidade e possibilidade, por si mesmas, mas foi em especial pela insatisfação da lógica classica em não oferecer uma análise satisfatória para implicação. Lewis não via a implicação como sendo a equivalência entre $\varphi \rightarrow \psi \mathrm{e} \neg(\varphi \wedge \neg \psi)$ ( $\varphi$ não ocorre simultaneamente a não $\psi$ ), mas como $\neg \diamond(\varphi \wedge \neg \psi)$ ou $\square(\varphi \rightarrow \psi)((\varphi$ não pode ocorrer com $\neg \psi)$ ou ( $\varphi$ necessariamente implica $\psi$ materialmente), respectivamente), conforme van Benthem [83, pág. 21].
} 
No curto período, do início do século $\mathrm{XX}$ até o final da década de sessenta, destacam-se os esforços de C. I. Lewis, J. Lukasevicz e Rudolf Carnap, responsáveis pelo cultivo e crescimento da lógica modal. Os trabalhos desse período caracterizam-se como sendo essencialmente sintáticos, isto é, a lógica proposicional clássica é enriquecida com operadores modais e através de um ou mais axiomas tenta-se subjazer a interpretação desejada (há a necessidade de uma semântica explícita). Esta visão sintática traz problemas como, por exemplo, o de distinguir sistemas lógicos, isto é, saber se ambos geram o mesmo conjunto de fórmulas.

Após essa fase evolutiva a lógica modal amadureceu graças às contribuições de Saul Kripke, juntamente com Arthur Prior, Stig Kanger, Jaakko Hintikka, J. C. C. Mackinsey, Alfred Tarski entre outros. Desenvolve-se a idéia da semântica dos mundos possiveis de Leibniz que por meio de Kripke apresentou-se frutífera para explicar noções "intensionais", consolidando-se definitivamente com as idéias de Church, quando da formulação de sua teoria dos tipos [13].

Noções "intensionais" e extensionais são devidas a Carnap sobre estudos dos trabalhos de Frege. Em geral, o uso do termo "intensional" se faz para indicar expressões em que os significados são levados em conta, isto é, há um agregado ao valor-verdade dessas expressões. Diz-se que uma expressão tem caráter "intensional" quando são referencialmente opacas, ou seja, as regras de substituição da lógica classica não se aplicam (Haddadi [33, pág. 14]). A partir dos estudos de Ruth B. Marcus, em que se combinam a teoria da quantificação com igualdade e a lógica modal, Quine formulou as noções de contextos referencialmente transparente e referencialmente opaco. $O$ primeiro refere-se às situações em que se podem aplicar as regras de substituição sem alterar a semântica, o segundo a contextos em que a permuta de idênticos provoca alterações da semântica.

Decorrente das discussões de questões inerentes à visão sintática surge a semântica em termos algébricos; o cálculo proposicional é encarado como sendo uma álgebra de alguma espécie e suas fórmulas vistas como sendo obtidas por operações algébricas. Resultados interessantes foram obtidos pelo uso dos métodos algébricos na tentativa de provar diferenças entre os sistemas de Lewis. Porém, as argumentações dessas discussões tinham caráter essencialmente semântico, isto é, envolviam conceitos de modelos, frames, satisfação e validade.

Os resultados de correção passam a diferenciar os vários sistemas modais, os de completude são caracterizados de modo extremamente simples, por exemplo: um determinado sistema modal pode ser completo se todas as suas fórmulas forem válidas em frames transitivos. Essa simplicidade, 
considerando-se as dificuldades do período sintático, em caracterizar sistemas modais dominaria as técnicas de trabalho pelos próximos quinze anos. Porém, surgem questões como: qualquer sistema modal normal é completo em relação a alguma classe de frames?

Nesse mesmo período e um pouco mais recente destacamos a corrente que defende a semântica em termos de modelos, cuja semântica dos mundos possíveis é a dominante ou a única conforme a interpretação que se dá ao termo mundo possível. Essa corrente inicia-se em 1972 (Blackburn [2]), ano em que S. K. Thomason mostra a existência de sistemas modais incompletos, isto é, a resposta a questão anterior é negativa. Em 1974, Thomason ([79] e [80]) e K. Fine ([27]) publicam exemplos de sistemas modais normais incompletos e uma série de artigos devido a Thomason mostra que a incompletude desses sistemas não é contornável.

Enquanto os resultados obtidos por Thomason estimularam uma análise mais aprofundada dos sistemas modais sobre o papel da linguagem modal (o que ela representa, de fato, na construção de um sistema formal modal?), outro fato que se destaca (próximo ao ano de 1972) é o uso das linguagens modais nas diversas áreas da ciência da computação.

O interesse pela expressividade da linguagem modal torna a teoria da correspondência um ramo de pesquisa da ciência da computação. Pratt ([69]) publica o trabalho seminal sobre o PDL. Ladner [48] mostra que o problema da satisfatibilidade para os sistemas modais entre $\mathrm{K}$ e $\mathrm{S} 4$ possui complexidade PSPACE-difícil. Resultados de Fischer e Ladner [29] e Pratt [68] mostram que o problema da satisfatibilidade do PDL é EXPTIME-completo.

Para nós um ganho considerável está no fato das linguagens modais deixarem de ser vistas como tendo caráter intrinsicamente "intensional". Elas se mostram naturalmente favoráveis para se lidar com qualquer estrutura relacional (Blackburn [2, pág. 39]), como é o caso do tratamento que damos a noção de conhecimento. As próximas seções tratam de alguns dos aspectos de como a noção intuitiva de conhecimento é capturada através de sistemas modais.

\subsection{Verdade, Conhecimento e Mundo Possível}

A teoria da verdade por correspondência fornece a noção clássica de verdade. Nela assume-se que: toda afirmação está correlacionada a um estado de desejos, isto é, uma sentença é verdadeira caso reflita o real, retrate aquilo que é e se isto não ocorrer ela é falsa (Tarski [78]). Os problemas relacionados a esta teoria como o da relação entre linguagem e realidade, o paradoxo do mentiroso, quando o estado de desejos refere-se ao próprio estado de 
desejos, entre outros está fora do escopo deste trabalho. O leitor poderá encontrar uma discussão sobre tais problemas em, por exemplo, Visser [86], da Costa [15] e Kirkham [47]. No entanto, fazemos alguns comentários para estabelecer parte do contexto em que desenvolvemos o trabalho.

$A$ verdade correspondencial vincula linguagem e realidade. A verdade de uma sentença $\varphi$, de uma linguagem, é função de como se interpreta esta linguagem, para da Costa [15, pág. 114]: uma teoria da correspondência, para ser filosoficamente satisfatória, carece deixar clara a indole da correspondência, que deve existir entre sentenças ou crenças, de um lado, e a realidade de outro que assegure a verdade. Porém, filosoficamente, verdade é indefinível por meio de conceitos mais simples, pois a própria sentença que a define, em sentido estrito, deve ser "verdadeira" (da Costa [15, pág. 115]).

Contudo, pela teoria da correspondência, qualquer definição ${ }^{8}$ para verdade deve satisfazer a seguinte condição: se $\varphi$ for uma proposição e $\bar{\varphi}$ seu nome, então: $\bar{\varphi}$ é verdadeira se e somente se $\varphi$. Essa foi a caracterização de Tarski para a noção de verdade, como correspondência.

Não se trata de uma definição propriamente dita, mas fornece condição sine qua non que qualquer definição deve satisfazer (da Costa [15, pág. 117]). É nesse contexto em que procuramos caracterizar a noção de conhecimento através da idéia de mundo possível.

Para nós, conhecer o significado de uma sentença é afirmar as condições sob as quais ela é verdadeira, ou seja, é saber como seria o mundo se ela fosse verdadeira. Essa correlação, em termos de mundos possiveis, fornece a seguinte idéia: o conhecimento corresponde a uma relação da qual se pode determinar em qual mundo nos encontramos (Halpern [36, pág. 323]). A idéia dessa semântica devido a Leibniz e primeiro ${ }^{9}$ formalizada por Hintikka ([42, pág. 93]), pode ser resumida do seguinte modo:

considere um agente $i$, localizado em um mundo $w$ (este é o mundo real de i). Seja $W$ um subconjunto do conjunto de todas as interpretações possíveis (de mundos possiveis ${ }^{10}$ ) de $w$ e $W^{\prime}$ $\left(W^{\prime} \subseteq W\right)$ as interpretações possíveis de $w$ segundo a percepção de $i$. Dizemos que $i$ conhece uma afirmação $\varphi$ em $w$, se e somente se, $\varphi$ se verifica em todas as interpretações que o agente tem de $w$, isto é, se $\bar{\varphi}$ é verdadeiro em todos os mundos contidos em $W^{\prime}$.

\footnotetext{
${ }^{8}$ Aqui definição significa o que caracteriza e esclorece.

${ }^{9}$ Kanger em 1957 explicitou uma semântica (standard) para a lógica modal (Hintikka [42, pág. 93]).

${ }^{10} \mathrm{Um}$ mundo possível não deve ser entendido como uma alternativa para o mundo real, mas como uma alternativa de alguma descrição do mundo real.
} 


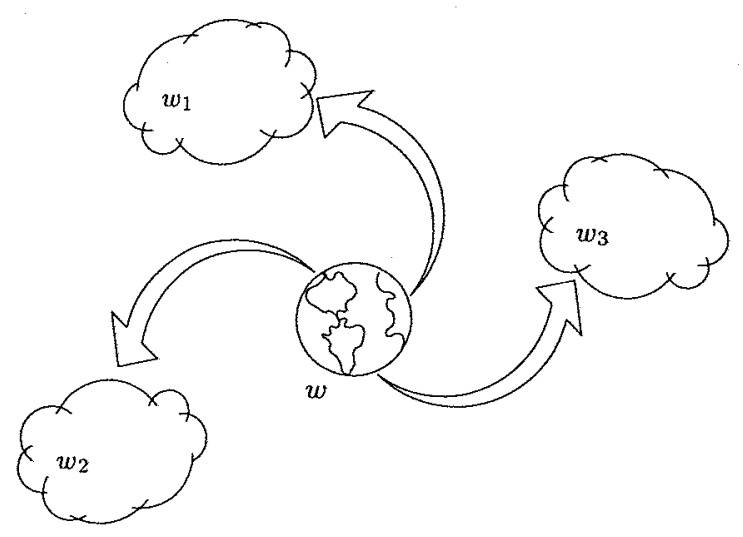

Figura 1.1: Os mundos possíveis a partir de $w$ segundo a visão do agente $i$.

O conhecimento de $i$ está em $W^{\prime}$ e depende única e exclusivamente do entendimento de $i$ sobre $w$. Isto é, o conhecimento expressa a atitude do agente em relação às suas interpretações do mundo real, assim uma semântica (para uma linguagem) que pretende capturar essa idéia de conhecimento deve estar associada a atitude do agente (ou agentes) em relação a interpretação (obtida diretamente da linguagem). Dessa forma, o conhecimento (como modalidade) é um modo sob a qual um objeto se relaciona com sua abstração, sua representação (vide exemplo 2.1).

Existe uma interconexão entre os mundos possíveis e as proposições primitivas: uma proposição pode ser falsa em um mundo e verdadeira em outro, mas para mantermos coesos os conceitos de conhecimento e mundo possível, este último deve possuir uma coerência interna ligada à estrutura meta-lógica das proposições (da Costa [15, pág. 150]). Portanto, um mundo possível pode ser caracterizado explicitamente como sendo formado por um conjunto maximal consistente dessas proposições (Ranta [70, pág. 77-79]). As noções de consistência e maximalidade não são as formais, porém possuem significado claro. Um conjunto de proposições é consistente se todas podem ser simultaneamente verdadeiras e maximal se ao adicionarmos qualquer outra proposição ao conjunto este se torna inconsistente. Esta forma explícita de caracterizar mundo possível pode ser encontrada em Hintikka ([41, pág. 153-157]), mas não nos trabalhos de Kripke.

No cálculo proposicional clássico não existe um mundo no qual uma proposição e sua negação sejam simultaneamente verdadeiras, pois para definir a verdade lógica no cálculo proposicional clássico não necessitamos saber como ela é considerada em um mundo particular, uma vez que estamos tratando simultaneamente de todos os mundos possíveis sob a percepção de um único agente. 
Por outro lado, ao considerarmos a percepção de vários agentes em mundos distintos surgem alguns problemas como, por exemplo: qual a razão de uma proposição ter valores diferentes em cada mundo possivel? $E$ devido a diferença de significados nos mundos ou simplesmente porque mudamos de mundo? Essas questões estão fora de nosso escopo. Uma discussão pode ser encontrada, por exemplo, em Zalta [89].

O assunto é de fato extenso e complexo, para o leitor interessado, dentre a vasta literatura, sugerimos da Costa [15], Gettier [31] e Lenzen [51]. Na próxima seção analisamos o cálculo proposicional clássico e sua semântica, uma vez que os sistemas modais são extensões de sua linguagem.

\subsection{O Cálculo Proposicional Clássico CP}

Para tratar $\varphi$, nas expressões $i$ sabe $\varphi$ ou $i$ conhece $\varphi$, estamos considerando um cálculo proposicional clássico (CP) tendo $\Phi$ como o conjunto de proposições primitivas referente a um certo domínio. Construimos CP definindo a parte sintática (cf. Hamilton [37, sec. 2.1], Bostok [4, sec. 5.2.2] e Mendelson [56, sec. 1.4]) e cujas expressões são conforme seguem:

Definição 1.1 (Elementos sintáticos de $\mathrm{CP}$ ).

Os elementos sintáticos de $\mathrm{CP}$ são definidos como segue:

1) $\Phi$ é um conjunto enumerável de símbolos proposicionais $p_{0}, p_{1}, p_{2}, \ldots$ Quando não houver problemas de ambigüidade utilizamos as letras $p$, $q$ e $r$ para representar os elementos de $\Phi$,

2) os símbolos para os conectivos são: $\neg e \rightarrow$,

3) símbolos auxiliares: parênteses à esquerda e à direita.

Definição $1.2(\mathrm{CP}(\Phi))$.

Uma fórmula atômica é um elemento de $\Phi$. $\mathrm{CP}(\Phi)$ é o menor conjunto contendo todas as atômicas e tal que se $\varphi$ e $\psi$ são elementos de $\mathrm{CP}(\Phi)$, então $(\neg \varphi)$ e $(\varphi \rightarrow \psi)$ também o sãa. Chamamos de fórmula às expressões em $\mathrm{CP}(\Phi)$. Definimos o tamanho de uma fórmula $\varphi$ pelo número de letras proposicionais e conectivos que nela ocorrem e denotamos por $|\varphi|$.

As únicas expressões que nos interessam são as fórmulas de $\mathrm{CP}$, resta estabelecer as formas de manipulação delas através de esquemas de axiomas $\mathrm{e}$ de uma única regra de inferência.

Definição 1.3.

Para quaisquer fórmulas $\varphi, \psi$ e $\chi$ são esquemas de axiomas de $\mathrm{CP}$ : 


$$
\begin{aligned}
& \left.1_{\mathrm{CP}}\right) \rightarrow(\psi \rightarrow \varphi) \\
& \left.2_{\mathrm{CP}}\right)(\varphi \rightarrow(\psi \rightarrow \chi)) \rightarrow((\varphi \rightarrow \psi) \rightarrow(\varphi \rightarrow \chi)) \\
& \left.3_{\mathrm{CP}}\right)(\neg \psi \rightarrow \neg \varphi) \rightarrow(\varphi \rightarrow \psi)^{11}
\end{aligned}
$$

$A$ regra de modus ponens (MP) é dada por: se $\varphi$ e $\varphi \rightarrow \psi$, então $\psi$, para $\varphi$ e $\psi$ em $\mathrm{CP}(\Phi)$. Esquematicamente: $\frac{\varphi \quad \varphi \rightarrow \psi}{\psi}$.

Cada instância de um dos esquemas de axioma é um axioma. Por abuso, chamamos esquemas de axiomas de axiomas. Os conectivos $\vee$ e $\wedge$ são definidos como abreviações a partir de $\neg \mathrm{e} \rightarrow$. Para quaisquer $\varphi$ e $\psi \in \operatorname{CP}(\Phi)$, $\varphi \vee \psi$ e $\varphi \wedge \psi$ são abreviações de $\neg \varphi \rightarrow \psi$ e $\neg(\varphi \rightarrow \neg \psi)$, respectivamente.

\section{Observação 1.1.}

Qualquer regra em um sistema $\mathrm{S}$ (formulada em sua meta-linguagem) que assegure que se as premissas são teoremas de $\mathrm{S}$, então a conclusão (obtida pela aplicação dessa regra) também é teorema de $\mathrm{S}$, é chamada de regra de inferência em S.

\section{Definição 1.4 (Substituição em CP).}

Uma substituição $\tau$ é uma função que associa a cada elemento de $\Phi$ uma fórmula em $\mathrm{CP}(\Phi)$. Escrevemos $\tau[\varphi]$ para indicar o resultado da substituição de cada proposiçâa primitiva $p_{j}$ que ocorre na fórmula $\varphi$ por $\tau\left(p_{j}\right)$.

Note que se $\tau$ é uma substituição e $\vdash \varphi$, então $\vdash \tau[\varphi]$. Há um processo, que chamamos prova, pelo qual podemos obter fórmulas de um determinado tipo em CP.

\section{Definição 1.5 (Prova em CP).}

Uma prova em CP é uma seqüência finita de fórmulas de $\varphi_{1}, \ldots, \varphi_{m}$ tal que para cada $j, 1 \leq j \leq m$ :

- $\varphi_{j}$ é um axioma de CP ou

- $\varphi_{j}$ é obtido por modus ponens a partir de fórmulas anteriores.

Se $\varphi_{1}, \varphi_{2}, \ldots, \varphi_{m}$ é uma prova em CP e $\varphi_{m}=\varphi$, então $\varphi_{1}, \varphi_{2}, \ldots, \varphi_{m}$ é uma prova de $\varphi$ em CP.

\section{Definição 1.6 (Teorema em CP).}

Um teorema de $\mathrm{CP}$ é uma fórmula $\varphi$ em $\mathrm{CP}$ tal que existe uma prova para $\varphi$ em CP. Se $\varphi$ é um teorema de $\mathrm{CP}$ denotamos este fato por $\vdash_{\mathrm{CP}} \varphi, e$ quando não houver problemas de ambigüidade escrevemos $\vdash \varphi$.

\footnotetext{
${ }^{11} 3_{\mathrm{CP}}$ pode ser substituido por $(\neg \varphi \rightarrow \neg \psi) \rightarrow((\neg \varphi \rightarrow \psi) \rightarrow \varphi$ ) (cf. Bostok [4, pág. 199] e Mendelson [56, pág. 32]).
} 
Cada prova de um teorema em CP gera uma árvore de prova correspondente, vejamos um exemplo:

\section{Proposição 1.1.}

i) Para qualquer $\varphi$ de $\mathrm{CP}(\Phi)$ temos que $\vdash \varphi \rightarrow \varphi$ em $\mathrm{CP}$.

ii) Para quaisquer $\varphi$ e $\psi$ de $\mathrm{CP}(\Phi)$ temos que $\neg \varphi \rightarrow(\varphi \rightarrow \psi)$ em $\mathrm{CP}$.

\section{Demonstração.}

i)

$$
\frac{\frac{\vdash \varphi \rightarrow((\varphi \rightarrow \varphi) \rightarrow \varphi) \quad \vdash(\varphi \rightarrow((\varphi \rightarrow \varphi) \rightarrow \varphi)) \rightarrow((\varphi \rightarrow(\varphi \rightarrow \varphi)) \rightarrow(\varphi \rightarrow \varphi))}{\vdash-(\varphi \rightarrow(\varphi \rightarrow \varphi)) \rightarrow(\varphi \rightarrow \varphi)}}{\vdash \varphi \rightarrow \varphi}
$$

ii)

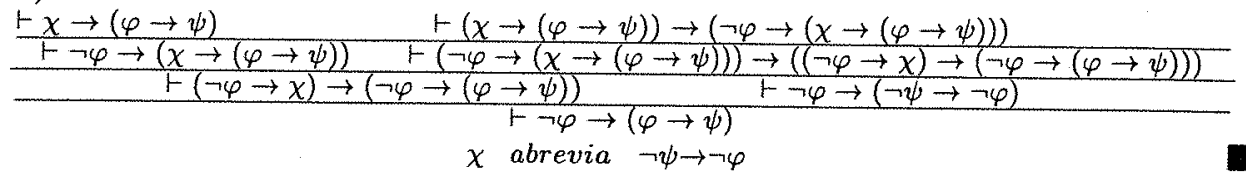

Aprimoramos a utilização das estruturas em árvores para efetuar provas, através do método de tableaux (seção 5.5).

Em CP, o vale meta-teorema da deduçãa ${ }^{12}$ (Herbrand 1930): se $\Gamma$ é um conjunto de fórmulas, $\varphi$ e $\psi$ são fórmulas $e \Gamma, \varphi^{13} \vdash_{\mathrm{CP}} \psi$, então $\Gamma \vdash_{\mathrm{CP}} \varphi \rightarrow \psi$. Em particular, se $\varphi \vdash_{\mathrm{CP}} \psi$, então $\vdash_{\mathrm{CP}} \varphi \rightarrow \psi$.

\section{Observação 1.2 .}

Apenas para lembrar, algumas tautologias de CP (cf. Hamilton [37] e Mendelson [56]):

$$
\begin{array}{lll}
\text { 1. } & \vdash_{K_{n}} \varphi \rightarrow \neg \neg \varphi, & \vdash_{K_{n}} \neg \neg \varphi \rightarrow \varphi \\
\text { 2. } \vdash_{K_{n}} \varphi \wedge \psi \rightarrow \varphi, & \vdash_{K_{n}} \varphi \wedge \psi \rightarrow \psi \\
\text { 3. } \vdash_{K_{n}} \varphi \rightarrow(\psi \rightarrow(\varphi \wedge \psi)) & \\
\text { 4. } & \vdash_{K_{n}}(\varphi \rightarrow \psi) \rightarrow(\neg \psi \rightarrow \neg \varphi) & \text { contra-positiva }
\end{array}
$$

Vale o silogismo hipotético (SH) em $\mathrm{K}_{n}$, isto é:

$$
\text { 5. se } \vdash_{\kappa_{n}} \varphi \rightarrow \psi \text { e } \vdash_{K_{n}} \psi \rightarrow \chi \text {, então } \vdash_{K_{n}} \varphi \rightarrow \chi
$$

$\mathrm{Na}$ seção seguinte discutimos a relação entre a linguagem de CP e sua semântica.

\footnotetext{
${ }^{12} \mathrm{~A}$ demonstração desse meta-teorema mostra que qualquer sistema $\mathrm{S}$ possui a noção de consequiência sintática a partir de premissas se: $1_{\mathrm{CP}}$ e $2_{\mathrm{CP}}$ são axiomas de $\mathrm{S}$ e modus ponens é a única regra de inferência de $\mathrm{S}$. A segunda condição é realmente fundamental, conforme Sundholm [77].

${ }^{13} \Gamma, \varphi$ abrevia $\Gamma \cup\{\varphi\}$
} 


\subsection{As Noções de Verdade em CP}

A construção de uma semântica para os elementos sintáticos de CP estabelece-se, em geral, a partir da noção de interpretaçãa ${ }^{14}$ que correlaciona elementos de $\Phi$ a $T$ ou $\perp$ (denotando verdadeiro e falso, respectivamente). Essa correlação possibilita definir a noção de conseqüência semântica $(\models)$ que permite tratar os conceitos de verdade e validade. Um dos objetivos da construção de um cálculo como CP é obter uma descrição sintática de $\models$ construindo um sistema formal cuja teoria da prova tenha bem definida a relação conseqüência sintática $(\vdash)$ e tal que suas propriedades operacionais espelham as de $\models$.

Em CP, esta correlação é determinada por meio de uma função de valoração $\pi^{15}$ de $\Phi$ em $\{T, \perp\}$; com a semântica é possível tratar os conceitos de verdade, validade e conseqüência lógica. Seja $I_{\Phi}$ o conjunto de todas as valorações de $\Phi$, I um elemento de $I_{\Phi}$ e $\varphi$ uma fórmula de CP, dizemos que $\varphi$ é verdadeiro sob uma valoraçấo I se $\mathrm{I}(\varphi)=T$ e denotamos por $\mathrm{I} F \varphi$. Uma fórmula $\varphi$ é válida na classe $I_{\Phi}$ se $\mathrm{I} \models \varphi$ para todo $\mathrm{I}$ em $I_{\Phi}$, e a denotamos por $I_{\Phi} \vDash \varphi$. Então, podemos dizer que o cálculo proposicional clássico possui dois níveis de verdade: a de verdade a partir de uma valoraçấo e a de validade lógica.

As regras inferência são fundamentais na análise entre $\vdash \mathrm{e} F$. Em Avron [1] encontramos mais de uma definição para regra de inferência, dentre as caracterizações encontradas na literatura consideramos as que dizem que uma inferência é uma regra que preserva a verdade ou que é uma regra que preserva a validade.

Grosso modo, podemos dizer que nas inferências que preservam a verdade está fixada uma interpretação e nas inferências que preservam a validade nada está fixado: ela trata de todas as interpretações possíveis. Mais precisamente, seja $\psi$ (cf. observação 1.1 - pág. 8) a expressão obtida pela aplicação de uma regra de inferência a partir da expressão $\varphi^{16}$, então as

\footnotetext{
${ }^{14} \mathrm{Na}$ prática, quando nos referimos à verdade ou à falsidade já temos implicitamente uma interpretação (um significado) pré-fixada (Tselishchev [82, pág. 392]). Logo, na construção de sistemas lógicos cada um dos elementos sintáticos, inclusive as regras de inferência tem pré-estabelecido um significado, que deve estar de acordo com a noção de verdade de Tarski.

${ }^{15}$ Pelo critério da composicionalidade podemos estender o domínio de $\pi$ para $\mathrm{CP}(\Phi)$; pois a semântica de uma fórmula depende unicamente da semântica atribuídas às suas partes, por exemplo, a semântica para a fórmula $(\varphi \wedge \psi)$ depende única e exclusivamente da semântica atribuída a $\varphi$ e $\psi$. Utilizando as bem conhecidas tabelas de verdade da lógica proposicional clássica.

${ }^{16} \varphi$ e $\psi$ são chamadas de premissa (ou antecedente) e conclusão (ou consequente), respectivamente.
} 
regras de inferência que preservam a verdade são tais que para toda substituição $\tau$ a veracidade de $\tau[\varphi]$ leva a veracidade de $\tau[\psi]$, isto é, para toda interpretação I de um conjunto específico $I\left(I \subseteq I_{\Phi}\right)$ e para todas as substituições $\tau$, se $I \models \tau[\varphi]$, então $I \models \tau[\psi]$. $\mathrm{E}$, as regras que preservam a validade são tais que para todo conjunto I de $I_{\Phi}$ e para toda substituição $r$ a validade de $\tau[\varphi]$ implica na validade de $\tau[\psi]$, isto é, para qualquer substituição $\tau$ se $I \models \tau[\varphi]$, então $I \models \tau[\psi]$.

Uma regra que preserva a verdade é $M P^{17}$. As regras de generalização universal da lógica de primeira ordem $\frac{\varphi}{\forall x \varphi}$ e a de necessitação (ou de generalização modal) da lógica modal: $\frac{\varphi}{\square} \varphi$ são exemplos de "regras de inferência" em que as verdades (como são) não são preservadas (cf. Fagin [23]), porém preservam a validade. Em [23], tais regras são ordenadas por $\subseteq$ quando vistas como relações sobre fórmulas, por exemplo, $M P$ relaciona conjuntos da forma $\{\varphi, \varphi \rightarrow \psi\}$ a fórmulas $\psi$.

Em CP regras que preservam validade preservam a verdade, isto é, as relações geram o mesmo conjunto de fórmulas, portanto os dois tipos de relações são idênticos, uma vez que toda regra que preserva verdade mantém a validade. Para a demonstração simulamos as funções de valoração em $C P$ através de substituições apropriadas.

Proposição 1.2 (Fagin [23]).

Em $\mathrm{CP}$ regras que preservam validade preservam verdade.

\section{Demonstração.}

Seja I uma interpretação de $I$ tal que $I(\varphi)=T$, devemos mostrar que $\mathrm{I}(\psi)=\mathrm{T}$. Seja $\tau$ uma substituição de modo que

$$
\tau\left(p_{j}\right)= \begin{cases}\top & \text { se } \mathrm{I}\left(p_{i}\right)=\top \\ \perp & \text { se } \mathrm{I}\left(p_{i}\right)=\perp\end{cases}
$$

logo para qualquer fórmula $\psi: \tau[\psi]$ é válida se e somente se $\mathrm{I}(\psi)=\mathrm{T}$. Por hipótese $\mathrm{I}(\varphi)=T$, então $\tau[\varphi]$ é válido. Ainda, como estamos supondo a preservação da validade, $\tau[\psi]$ é válido, portanto $\mathrm{I}(\psi)=\mathrm{T}$.

No cálculo de predicados clássico as noções de verdade de CP necessitam ser redefinidas, isto é feito a partir de um terceiro conceito que não ocorre no cálculo proposicional, a satisfação.

Vejamos como entendemos tais diferenças. Suponha que $\rho_{1}(x)$ equivale a dizer que " $x$ é branco". A instanciação de $x$ por uma constante a nos fornece uma proposição $\rho_{1}(\mathrm{a})$ que em CP pode ser classificada como

\footnotetext{
${ }^{17}$ Podemos entender a regra $M P$ em CP não como a forma de uma inferência, mas como um método explícito de enumerar as verdades lógicas de CP (Hintikka [44, cap. 1]).
} 
sendo verdadeira ou falsa. $O$ valor-verdade correspondente depende da constante a e conforme discussão na seção 1.2 ela se estabelece por métodos extra-lógicos.

Para dizer se a proposição $\rho_{1}(\mathrm{a})$ é verdadeira ou não, no caso do cálculo de predicados, não podemos simplesmente associar uma interpretação ao predicado e um objeto à constante. Parte do problema está em saber como o predicado está associado ao objeto ou em saber com quais objetos está associado. $\mathrm{O}$ conjunto dos objetos eleitos por tal associação é dito ser o domínio da interpretação.

Considere como domínio o conjunto dos humanos, tomemos o predicado $\rho_{2}(x y)$ como sendo " $x$ ama $y^{\prime \prime}$ e sejam b e c as constantes denotando "Beto" e "Carla", então $\rho_{2}(\mathrm{bc})$ significa "Beto ama Carla". Veja que a ordem dos argumentos pode alterar o valor do predicado, isto é $\rho_{2}(\mathrm{bc})$ e $\rho_{2}(\mathrm{cb})$ podem não ter o mesmo valor-verdade. Logo, não é o conjunto dos objetos (associados ao predicado) que determina o valor-verdade, mas a seqüência em que ela está associada ao predicado. Dizemos então que $\rho_{2}(x y)$ é satisfativel pela seqüência bc, mas pode não o ser por cb. Como pode não valer para toda seqüência não é nem uma verdade, nem algo logicamente válido.

Agora, considere $\rho_{3}(x y z)$ como sendo " $x, y$ e $z$ são mortais", desde que todos os humanos são mortais o predicado é satisfeito para qualquer seqüência do domínio. E $\rho_{3}(x y z)$ é verdadeira, pois existe de uma interpretação na qual o predicado é satisfeito para qualquer seqüência sob a interpretação fixada, mas $\rho_{3}(x y z)$ não é logicamente válida.

Por fim, tomemos o predicado $\rho_{3}(x y z) \rightarrow \rho_{3}(x y z)$. Esta expressão é logicamente válida, pois é verdadeira não só para a seqüência de todos os humanos mas para qualquer seqüência de quaisquer objetos em qualquer domínio.

Resumindo, uma fórmula $\varphi$ é satisfativel se e somente se existe uma interpretação na qual há uma seqüência de objetos no domínio tal que $\varphi$ se verifica. Uma fórmula $\varphi$ é verdadeira por alguma interpretação se e somente se para toda sequência de objetos no domínio da interpretação $\varphi$ é satisfeita. E, $\varphi$ é válida (ou é uma tautologia) se é verdadeira para toda interpretação. Utilizamos essas noções no próximo capítulo (seções $2.2 \mathrm{e}$ 2.3) em que estudamos a semântica dos sistemas de conhecimento. 


\section{$\mathrm{O}$ sistema $\mathrm{K}_{n}$}

Neste capítulo, apresentamos o sistema de conhecimento $\mathrm{K}_{n}$ obtido a partir do sistema modal K. Seguimos o formato de textos clássicos como Bull e Segerberg [5], Chellas [11] e Hugues e Cresswell [17]. Ao axiomatizarmos o conhecimento de um agente estamos interessados sobretudo em questões computacionais, fica fora do escopo discutir questões como: o conhecimento pode ser axiomatizado? ou quais os axiomas que melhor caracterizam o conhecimento dos agentes?

Inicialmente, na seção 2.1, definimos uma linguagem formal do sistema $\mathrm{K}_{n}$, com letras proposicionais, conectivos lógicos, operadores modais e regras de manipulação das expressões da linguagem.

A seguir, na seção 2.2, estudamos a semântica na qual definimos estruturas e classes de estruturas baseadas numa relação que associa elementos extra-lingüísticos às expressões da linguagem.

Finalmente, nas seções 2.3 e 3.1 , fazemos um estudo sobre as relações entre a parte sintática e sua respectiva semântica.

\subsection{O Sistema $K_{n}$}

Nesta seção formalizamos a linguagem e a gramática necessárias para tratar o conceito de conhecimento conforme a noção intuitiva anteriormente caracterizada (cf. seção 1.2), isto é, definimos o sistema $\mathrm{K}_{n}$ e o operador modal $K_{i}$ cuja forma básica é $K_{i} \varphi$ que abrevia a expressão $i$ conhece $\varphi$ (ou $i$ sabe $\varphi$ ). Vale observar que $\mathrm{K}_{n}$ nos fornece uma visão esquemática do conhecimento em um determinado momento, isto é, engloba num determinado momento o conhecimento de $i$ referente a um certo domínio. 
Definimos o sistema $\mathrm{K}_{n}$ como segue:

\section{Definição 2.1.}

Os elementos sintáticos de $\mathrm{K}_{n}$ :

$1_{\mathrm{K}_{n}}$ ) estamos considerando a linguagem de $\mathrm{CP}$, e um conjunto finito de agentes $\Delta=\{1,2, \ldots, n\}$ (se $\Delta=\{1\}$ temos $\mathrm{K}_{n}=\mathrm{K}$ );

$\left.2_{\mathrm{K}_{n}}\right) \mathrm{K}_{n}(\Phi)$ é o menor conjunto contendo $\mathrm{CP}(\Phi)$, tal que se $\varphi, \psi \in \mathrm{K}_{n}(\Phi)$, então $(\neg \varphi),(\varphi \rightarrow \psi)$ e $\left(K_{i} \varphi\right)$ também são elementos de $\mathrm{K}_{n}(\Phi)$, para $i \in \Delta$.

$3_{\mathrm{K}_{n}}$ ) Um conjunto de esquemas de axiomas.

- todas as tautologias de $\mathrm{CP}$,

- $K_{i}(\varphi \rightarrow \psi) \rightarrow\left(K_{i} \varphi \rightarrow K_{i} \psi\right)$, para $\varphi, \psi \in \mathrm{K}_{n}(\Phi)$ e $i \in \Delta$.

Note que em $K_{i} \varphi \rightarrow K_{i} \psi$ o símbolo " $\rightarrow$ " estende aquele definido em CP.

$\mathrm{O}$ esquema de axioma $K_{i}(\varphi \rightarrow \psi) \rightarrow\left(K_{i} \varphi \rightarrow K_{i} \psi\right)$ é chamado de $\mathrm{Ke}$ pode ser lido como: se $i$ conhece $\varphi \rightarrow \psi$, então se $i$ conhecer $\varphi$, $i$ conhece $\psi$. Em termos dos mundos possíveis de $i$ significa que se $\varphi \rightarrow \psi$ é verdadeira em todos os mundos possíveis de $i$, então se $\varphi$ é verdadeira em todos os mundos possiveis, $\psi$ também o é, ou seja o conhecimento de um agente é fechado pela implicação.

Os conceitos de teorema e substituição em $\mathrm{K}_{n}$ são introduzidos mutatis mutandis como feito em CP. Além da regra $M P$ (adequada a $\mathrm{K}_{n}$ ), temos a regra de generalização modal ( $c f$. Observação 1.1).

\section{Definição 2.2 (GM).}

A regra de generalização GM: $\frac{\varphi}{K_{i} \varphi}$.

GM pode ser entendida como tudo que é derivável de verdades conhecidas é conhecimento verdadeiro (teoremas são verdades conhecidas).

Os operadores modais funcionam como uma espécie de quantificador universal restrito (vide observação 3.2 ), assim sua inclusão pode ser considerada uma forma de generalização, as propriedades desses operadores modais encontram-se caracterizados pela definição 2.6 de satisfatibilidade e pelo teorema 2.7, que caracteriza a satisfatibilidade em classes de estruturas. Ainda, os agentes são elementos ideais, pois a partir do axioma $K$ e de $G M$, o agente conhece todas as conseqüências lógicas de $K_{n}$.

Em $\mathrm{K}_{n}$, vale o meta-teorema da dedução como em $\mathrm{CP}$, desde que não seja utilizada GM em nenhuma premissa, conforme Nota 12 . Vejamos um exemplo: 


\section{Proposição 2.1.}

Para quaisquer fórmulas $\varphi$ e $\psi$ em $\mathrm{K}_{n}(\Phi)$ valem:

$\vdash_{\kappa_{n}}\left(K_{i} \varphi \wedge K_{i} \psi\right) \rightarrow K_{i}(\varphi \wedge \psi) \quad e \quad \vdash_{\mathrm{K}_{n}} K_{i}(\varphi \wedge \psi) \rightarrow\left(K_{i} \varphi \wedge K_{i} \psi\right)$

Demonstração. [Nolt [63, sec. 10.7]]

Demonstramos apenas o primeiro item, para o segundo veja a referência.

1. $\vdash K_{i} \varphi$

2. $\vdash K_{i} \psi$

3. $\vdash \varphi \rightarrow(\psi \rightarrow(\varphi \wedge \psi))$

Premissa

4. $\vdash K_{i}(\varphi \rightarrow(\psi \rightarrow(\varphi \wedge \psi)))$

Premissa

5. $\vdash K_{i}(\varphi \rightarrow(\psi \rightarrow(\varphi \wedge \psi)))$

Teor. de CP

6. $\vdash K_{i} \varphi \rightarrow K_{i}(\psi \rightarrow(\varphi \wedge \psi))$

7. $\vdash K_{i}(\psi \rightarrow(\varphi \wedge \psi)) \rightarrow\left(K_{i} \psi \rightarrow K_{i}(\varphi \wedge \psi)\right)$

GM 3

$K$

8. $\vdash K_{i} \varphi \rightarrow\left(K_{i} \psi \rightarrow K_{i}(\varphi \wedge \psi)\right)$

$M P \quad 4,5$

$K$

9. $\vdash K_{i} \psi \rightarrow K_{i}(\varphi \wedge \psi)$

10. $\vdash K_{i}(\varphi \wedge \psi)$

$\begin{array}{ll}S H & 6,7\end{array}$

$M P \quad 1,8$

$M P 2,9$

Pelo meta-teorema da dedução decorre que $\vdash_{K_{n}}\left(K_{i} \varphi \wedge K_{i} \psi\right) \rightarrow K_{i}(\varphi \wedge \psi)$.

Nos casos em que se utiliza $G M$ em premissas introduzimos a seguinte definição (Sundholm [77]):

\section{Definição 2.3 .}

A dedução (ou prova a partir de premissas) em $\mathrm{K}_{n}$ é como segue:

$$
\Gamma \vdash_{\kappa_{n}} \varphi \text { se, e somente se, } \vdash_{\kappa_{n}} \varphi_{1} \rightarrow \varphi_{2} \rightarrow \cdots \rightarrow \varphi_{j} \rightarrow \varphi,
$$

$\operatorname{com} \varphi_{1}, \varphi_{2}, \ldots, \varphi_{j} \in \Gamma$

$\varphi_{1} \rightarrow \varphi_{2} \rightarrow \cdots \rightarrow \varphi_{j} \rightarrow \varphi$ abrevia $\varphi_{1} \rightarrow\left(\varphi_{2} \rightarrow\left(\cdots \rightarrow\left(\varphi_{j} \rightarrow \varphi\right) \cdots\right)\right)$ que pode ser escrito como $\left(\varphi_{1} \wedge \varphi_{2} \wedge \cdots \wedge \varphi_{j}\right) \rightarrow \varphi$. Veja que, pela definição acima, o teorema da dedução continua valendo em $\mathrm{CP}$, pois $\varphi_{1} \wedge \ldots \wedge \varphi_{j} \wedge \varphi \rightarrow \psi$ se, e só se, $\varphi_{1} \wedge \ldots \wedge \varphi_{j} \rightarrow(\varphi \rightarrow \psi)$. Está fora de nossos objetivos um estudo sobre as regras de inferência, ao leitor interessado indicamos os textos de Sundholm [77] e Fagin [23].

A inferência a partir de premissas permite provar que conhecimentos de fatos contraditórios levam os agentes a conhecer tudo, isto é, se para algum $\varphi$ em $\mathrm{K}_{n}(\Phi)$ tem-se que $K_{i} \varphi$ e $K_{i}(\neg \varphi)$, então $K_{i} \psi$, para qualquer $\psi$ em $\mathrm{K}_{n}(\Phi)$. Para verificar esta afirmação utilizamos a seguinte proposição.

\section{Proposição 2.2.}

$$
\vdash\left(K_{i} \varphi \wedge K_{i} \neg \varphi\right) \rightarrow K_{i} \psi \text {. }
$$




\section{Demonstração.}

1. $\vdash(\varphi \wedge \neg \varphi) \rightarrow \psi$

Prop. 1.1.ii

2. $\vdash K_{i}((\varphi \wedge \neg \varphi) \rightarrow \psi)$

3. $\vdash K_{i}((\varphi \wedge \neg \varphi) \rightarrow \psi) \rightarrow\left(K_{i}(\varphi \wedge \neg \varphi) \rightarrow K_{i} \psi\right)$

GM em 1

4. $\vdash K_{i}(\varphi \wedge \neg \varphi) \rightarrow K_{i} \psi$

$K$

5. $\vdash\left(K_{i} \varphi \wedge K_{i} \neg \varphi\right) \rightarrow\left(K_{i}(\varphi \wedge \neg \varphi)\right)$

$M P \quad 2,3$

6. $\vdash\left(K_{i} \varphi \wedge K_{i} \neg \varphi\right) \rightarrow K_{i} \psi$

Prop. 2.1

SH 4,5 .

Da proposição anterior e pela definição de dedução (def. 2.3) decorre que: se $\Gamma=\left\{K_{i} \varphi, K_{i}(\neg \varphi)\right\}$, então $\Gamma \vdash K_{i} \psi$, para qualquer $\psi$ em $\mathrm{K}_{n}(\Phi)$. Seguindo a notação de $C P$, toda fórmula $\varphi$ de $\mathrm{K}_{n}(\Phi)$ que possui uma dedução em $\mathrm{K}_{n}$ é chamada de $\mathrm{K}_{n}$-dedutível (ou teorema de $\mathrm{K}_{n}$ ) e denotamos por $\vdash_{K_{n}} \varphi$ (ou simplesmente $\vdash \varphi$ ). A seguir algumas notações e propriedades de acordo com o conceito de dedução.

\section{Definição 2.4.}

Uma fórmula $\varphi$ é consistente em $\mathrm{K}_{n}$ (ou $\mathrm{K}_{n}$-consistente) se, e só se, $\neg \varphi$ não é $\mathrm{K}_{n}$-dedutivel. Um conjunto $\left\{\varphi_{1}, \ldots, \varphi_{m}\right\}$ de fórmulas é $\mathrm{K}_{n}$-consistente se, e só se, $\varphi_{1} \wedge \cdots \wedge \varphi_{m}$ o é. Um conjunto infinito de fórmulas é $\mathrm{K}_{n}$ consistente se todos os seus subconjuntos finitos o são. Denotamos um conjunto $\Sigma$ consistente por $\Sigma_{\mathrm{con}}$. Uma fórmula ou conjunto de fórmulas $e ́$ inconsistente se não for consistente. Um conjunto $\Sigma$ é maximal consistente $\left(\Sigma_{\operatorname{maxc}}\right)$ se é consistente e para qualquer $\varphi(\varphi \notin \Sigma)$, o conjunto $\Sigma \cup\{\varphi\}$ é inconsistente.

\section{Proposição 2.3.}

Seja S um sistema que inclui todas as tautologias de CP e MP, então:

i) todo conjunto consistente $\Gamma$ pode ser estendido a um conjunto $\Sigma$ tal que $\Gamma \subseteq \Sigma e \Sigma$ é maximal consistente em $\mathrm{S}$,

ii) dados $\Sigma_{\operatorname{maxc}}$ e $\psi$, só uma das fórmulas $\psi$ ou $\neg \psi$ pertence à $\Sigma_{\operatorname{maxc}}$,

iii) $\varphi \wedge \psi \in \Sigma_{\operatorname{maxc}}$ se e somente se $\varphi \in \Sigma_{\operatorname{maxc}} e \psi \in \Sigma_{\operatorname{maxc}}$.

iv) $\varphi \rightarrow \psi \in \Sigma_{\operatorname{maxc}}$ se e somente se $\neg \varphi \in \Sigma_{\operatorname{maxc}}$ ou $\psi \in \Sigma_{\operatorname{maxc}}$.

v) se $\varphi \in \Sigma_{\operatorname{maxc}} e(\varphi \rightarrow \psi) \in \Sigma_{\operatorname{maxc}}$, entäo $\psi \in \Sigma_{\operatorname{maxc}}$,

vi) se $\varphi$ é teorema de $\mathrm{S}$, então $\varphi \in \Sigma_{\operatorname{maxc}}$.

\section{Demonstração. (Chang e Keisler [10, págs. 9-10])}

i) Este fato é conhecido como lema de Lindenbaun e sua demonstração é como segue: enumeramos todas as fórmulas de $S$ (p.ex., cf. Hunter [46, págs. 108-109]): $\varphi_{0}, \varphi_{1}, \ldots, \varphi_{m}, \ldots$ Construímos uma seqüência 
crescente de conjuntos consistentes $\Gamma=\Gamma_{0} \subseteq \Gamma_{1} \subseteq \cdots \subseteq \Gamma_{m} \subseteq \cdots$, do seguinte modo: se $\Gamma_{0} \cup\left\{\varphi_{0}\right\}$ é consistente, $\Gamma_{1}=\Gamma_{0} \cup\left\{\varphi_{0}\right\}$ senão $\Gamma_{1}=\Gamma_{0} . \mathrm{E}$, se $\Gamma_{m} \cup\left\{\varphi_{m}\right\}$ é consistente, então $\Gamma_{m+1}=\Gamma_{m} \cup\left\{\varphi_{m}\right\}$ senão $\Gamma_{m+1}=\Gamma_{m}$. Seja $\Sigma=\bigcup_{j \in \mathbb{N}} \Gamma_{j}$.

O conjunto $\Sigma$ é consistente. De fato: suponha que não, então existe $\left\{\psi_{1}, \psi_{2}, \ldots, \psi_{m}\right\}$ subconjunto de $\Sigma$ tal que $\vdash_{s} \neg\left(\psi_{1} \wedge \psi_{2} \wedge \cdots \wedge \psi_{m}\right)$. Considere $\theta_{0}, \theta_{1}, \ldots, \theta_{l}$ todas as fórmulas dessa dedução, pela construção de $\Sigma$ existe $\Gamma_{j}$ tal que $\left\{\psi_{1}, \psi_{2}, \ldots, \psi_{m}, \theta_{0}, \theta_{1}, \ldots, \theta_{l}\right\} \subseteq \Gamma_{j}$. Como $\left\{\psi_{1}, \psi_{2}, \ldots, \psi_{m}\right\} \subseteq \Gamma_{j}$ e $\vdash_{s} \neg\left(\psi_{1} \wedge \psi_{2} \wedge \cdots \wedge \psi_{m}\right)$, então $\Gamma_{j}$ é inconsistente, o que contradiz a construção de $\Gamma_{j}$.

$\Sigma$ é maximal, pois se $\Sigma^{\prime}$ é consistente e tal que $\Sigma \subseteq \Sigma^{\prime} \operatorname{com} \psi$ em $\Sigma^{\prime}$, então como toda fórmula de $S$ foi numerada existe $\varphi_{m}$ que corresponde a $\psi$. Logo, $\Gamma_{m} \cup\left\{\varphi_{m}\right\}$ é consistente e $\psi \in \Sigma$.

ii) Para a demonstração recorremos à enumeração e à construção da seqüência de conjuntos consistentes do item anterior. Assim, $\psi$ e $\neg \psi$ entram na construção dos conjuntos consistentes do seguinte modo: se $\Gamma_{j} \cup\{\psi\}$ é consistente, então $\psi \in \Sigma_{\operatorname{maxc}}$ e $\forall \neg \neg \psi$ em S; por outro lado se $\Gamma_{m} \cup\{\neg \psi\}$ é consistente, $(\neg \psi) \in \Sigma_{\operatorname{maxc}}$ e $\forall \neg \neg \neg \psi$ em $\mathrm{S}$, isto é, $\forall \psi$ em $\mathrm{S}^{1}$. Logo, não pode ocorrer de $\psi$ e $\neg \psi$ pertencerem à $\Sigma_{\operatorname{maxc}}$, pois contraria a consistência de $\Sigma_{\operatorname{maxc}}$ uma vez que $\{\psi, \neg \psi\} \subseteq \Sigma_{\operatorname{maxc}} \mathrm{e} \vdash_{\mathrm{s}} \psi \rightarrow \psi^{2}$.

iii) Supondo $\varphi \wedge \psi \in \Sigma_{\operatorname{maxc}}$ e $\neg \varphi \in \Sigma_{\operatorname{maxc}}$, então $\{\neg \varphi, \varphi \wedge \psi\} \subseteq \Sigma_{\operatorname{maxc}}$. Porém, $\neg(\neg \varphi \wedge \varphi \wedge \psi)$ é abreviação de $\varphi \rightarrow(\psi \rightarrow \varphi)$, que é instância de $1_{\mathrm{CP}}$ (tautologia de CP), e $\vdash_{s} \varphi \rightarrow(\psi \rightarrow \varphi)$. Como $\Sigma_{\operatorname{maxc}}$ é consistente segue que $\neg \varphi \notin \Sigma_{\operatorname{maxc}}$ e pelo item anterior $\varphi \in \Sigma_{\operatorname{maxc}}$. Analogamente, verifica-se que $\psi \in \Sigma_{\operatorname{maxc}}$. Para a recíproca o procedimento é análogo, basta supor que $\neg(\varphi \wedge \psi) \in \Sigma_{\operatorname{maxc}}$, isto é, $\{\varphi, \psi, \neg(\varphi \wedge \psi)\} \subseteq \Sigma_{\operatorname{maxc}}$ que fere a consistência de $\Sigma_{\operatorname{maxc}}$ e $\varphi \wedge \psi \in \Sigma_{\operatorname{maxc}}$.

iv) Segue diretamente de (iii), pois $\varphi \rightarrow \psi$ pode ser escrito como $\neg(\varphi \wedge \neg \psi)^{3}$, então $(\varphi \rightarrow \psi) \in \Sigma_{\operatorname{maxc}}$ se e só se $\neg \varphi \in \Sigma_{\operatorname{maxc}}$ ou $\psi \in \Sigma_{\operatorname{maxc}}$.

$v$ ) Pelo item anterior e da hipótese de que $\varphi \in \Sigma_{\operatorname{maxc}}$, então $\psi \in \Sigma_{\operatorname{maxc}}$.

vi) Finalmente, estamos supondo $\vdash_{5} \varphi$. De $(i i)$, se $\neg \varphi$ pertence à $\Sigma_{\operatorname{maxc}}$, então $\Sigma_{\operatorname{maxc}}$ é inconsistente, portanto $\varphi \in \Sigma_{\operatorname{maxc}}$.

\footnotetext{
${ }^{1}$ Vide observação 1.2.

${ }^{2} \vdash_{5} \psi \rightarrow \psi$ pela proposição em 1.1 e $\psi \rightarrow \psi$, pode ser escrito como $\neg(\psi \wedge \neg \psi)$.

${ }^{3}$ Pela dupla-negaçâo vide observação 1.2
} 
As noções associadas à consistência serão relacionadas (cf. teoremas 2.6 e 2.7) às de satisfatibilidade e validade, de acordo com a semântica atribuida aos elementos de $\mathrm{K}_{n}$. A próxima seção introduz uma semântica que atribui ao operador $K_{i}$ a noção de conhecimento.

\subsection{Semântica para $\mathrm{K}_{n}$ : satisfatibilidade e validade}

A semântica para $K_{n}$ é caracterizada pela noção de mundo possível (conforme o capítulo anterior) e engloba a semântica de CP. Iniciamos definindo o conceito de interpretação modal que é uma extensão da noção de interpretação em $\mathrm{CP}$, uma vez que uma bivaloração corresponde (intuitivamente) a uma certa descrição da realidade (a um mundo possível). Como a interpretação em CP é dada por uma função de valoração e desejamos estender essa função, uma questão pode ser levantada de imediato: quais os elementos do domínio dessa nova função? Dunn ([20, sec. 2,3]) oferece duas opções: (i) todas a fórmulas de $\Phi^{4}$ mais as modais irredutíveis ${ }^{5}$ pertencem ao domínio da função, ou (ii) o domínio é composto somente pelas fórmulas em $\Phi$, isto é, não existem fatos modais irredutíveis no domínio.

Ficamos com a opção (ii) para conservar a noção de interpretação em CP. Um outro fato que nos leva a adotar esta opção é dado no Exemplo 2.2 , logo após a definição de satisfatibilidade. Assim, uma interpretação modal é uma função de $\Phi \mathrm{em}\{\perp, T\}$ referente a um mundo $w$. A bivaloração não fere a caracterização dada por Hintikka em termos de consistência e maximalidade.

Note que ao nos referirmos a um mundo possível $w$, estamos nos referindo a uma possível interpretação modal que identificamos por $\pi_{w}$, isto é, dizer que existe um mundo possível $w$ significa dizer que existe uma possivel interpretação $\pi_{w}$ das proposições primitivas que compõe $w$ que pode ser qualquer uma entre as $2^{|\Phi|}$ existentes. Se $W$ é um subconjunto do conjunto de todos os mundos possíveis referentes ao domínio de $\Phi$, então $W$ possui $|W|$ referências e cada uma delas com $2^{|\Phi|}$ possibilidades de escolha.

A noção intuitiva de conhecimento se efetiva através do conceito de validade. Para isso, são cruciais as relações $\mathcal{K}_{i}\left(\mathcal{K}_{i} \subseteq W \times W, i \in \Delta\right)$ entre o "mundo real" $w_{0}$ e suas possíveis descrições alternativas, de acordo com a visão de $i$ sobre $w_{0}$. Vejamos uma situação cuja representação é feita por uma estrutura relacional envolvendo a idéia de mundos possíveis.

\footnotetext{
${ }^{4}$ Basta considerar o conjunto $\Phi$ uma vez que a valoração de qualquer fórmula de $C P(\Phi)$ pode ser obtida a partir da definição dos conectivos $\neg \mathrm{e} \rightarrow$.

${ }^{5}$ Fórmulas da forma $K_{i} \varphi \operatorname{com} \varphi \in \Phi$.
} 


\section{Exemplo 2.1 (Muddy children Puzzle (cf. Fagin et al., [24])).}

Considere $n$ crianças brincando todas juntas, ao final de um dia de diversão e apesar de todo o cuidado tomado para não se sujarem algumas (digamos $k$ ) crianças acabam se sujando e justamente suas testas, onde não podem ver sua própria sujeira. Antes de irem para o banho, o pai das crianças informa-as (em alto e bom tom): "pelo menos um de vocês está com a testa suja". E pergunta repetidas vezes (até obter a resposta): "quem sabe que está com a própria testa suja?"

Para uma representação adequada devemos levar em consideração que desejamos retratar uma situação em que a informação a ser obtida é se a uma criança está ou não com a testa suja. Então, a descrição de uma situação possivel é dada por um conjunto $\Phi$ de proposições primitivas $p_{i}$ que afirmam: $i$ está com a testa suja, com $i$ indicando uma das $n$ crianças, isto é, cada mundo possivel é composto por proposições afirmando se i está ou não com a testa suja. Podemos fixar $x_{i}=1$ se a criança $i$ está com a testa suja e $x_{i}=0$ caso contrário, logo a n-upla $\left(x_{1}, x_{2}, \ldots, x_{n}\right)$ de $0^{\prime} s$ e $1^{\prime} s$ representa um mundo possivel $w$. Assim, uma interpretação modal referente a um mundo $w$ (para uma n-upla) é uma função tal que $\pi_{w}\left(p_{i}\right)=$ T se $x_{i}=1$ e $\pi_{w}\left(p_{i}\right)=\perp$ se $x_{i}=0$. A afirmação feita pelo pai pode ser representada por $p_{1} \vee p_{2} \vee \cdots \vee p_{n}$, com $\pi_{w}\left(p_{1} \vee \cdots \vee p_{n}\right)=T$. As possibilidades quanto a uma criança $i$ saber se sua própria testa está suja ou não se estabelece pelas relações entre os mundos, isto é, dois mundos se relacionam se eles mantém uma coerência ${ }^{6}$ segundo i. Graficamente:

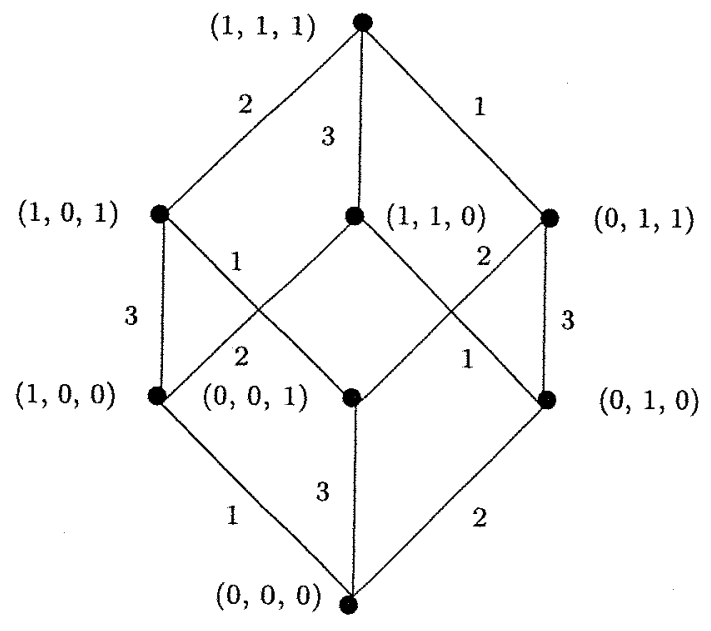

Figura 2.1: Estrutura relacional do "muddy children puzzle", para $n=3$.

\footnotetext{
${ }^{6} \mathrm{~A}$ coerência entre dois mundos é mantida se $i$ enxerga as mesmas crianças sujas nos dois mundos, a exceção de sua própria sujeira.
} 
A figura anterior representa a situação antes do pai se pronunciar, cada vértice é um mundo possível e as arestas as relações $\mathcal{K}_{i}^{7}$ entre mundos. As relaçães são de acordo com a visão de $i$, note que a partir de um mundo $w=\left(\mathrm{t}_{1}, \mathrm{t}_{2}, \ldots, \mathrm{t}_{i-1}, 1, \mathrm{t}_{i+1}, \ldots, \mathrm{t}_{n}\right)$ a criança $i$ tem acesso aos mundos $w^{\prime}=$ $\left(t_{1}, t_{2}, \ldots, t_{i-1}, 0, t_{i+1}, \ldots, t_{n}\right)$ e ao próprio $w$. Abaixo a representação da situação após o pai informar que "pelo menos um deles está com a testa suja".

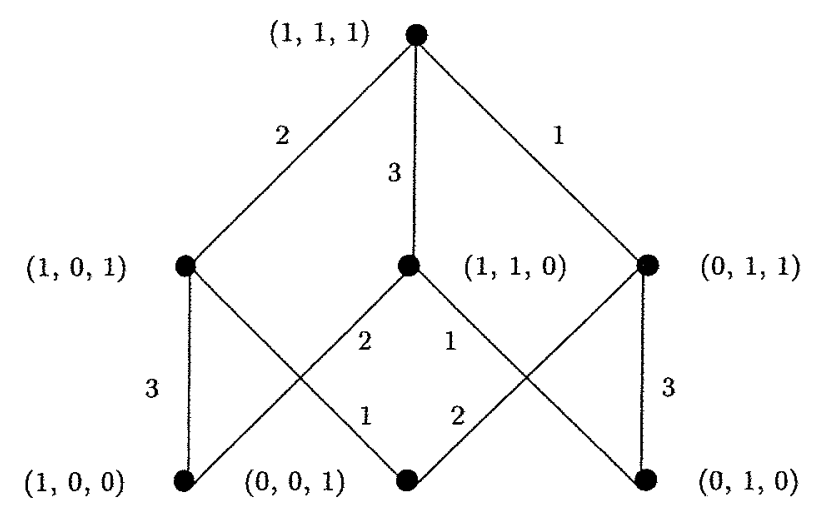

Figura 2.2: A situação após a informação do pai.

A seguir formalizamos as noções de satisfatibilidade e validade por meio das estruturas de Kripke.

\section{Definição 2.5.}

Um frame (ou molde) F para $\Delta=\{1,2, \ldots, n\}$ é uma $(n+1)$-upla $\left\langle W, \mathcal{K}_{1}, \ldots, \mathcal{K}_{n}\right\rangle$. Com $W$ um conjunto de mundos possiveis e $\mathcal{K}_{i}$ relações sobre $W$ para cada $i \in \Delta$. Uma estrutura de Kripke $\mathrm{M}$ é uma $(n+2)$ upla $\left\langle W, \pi_{W}, \mathcal{K}_{1}, \ldots, \mathcal{K}_{n}\right\rangle$. Com $\pi_{W}$ o conjunto de todas as interpretações modais ${ }^{8}$ referentes aos mundos em $W$ e $\mathcal{K}_{i}$ relações sobre $W$. Uma estrutura $\mathrm{M}$ é baseado num frame $F$ se o conjunto $W$ e as relaçôes $\mathcal{K}_{i}$ em $\mathrm{M}$ são as de $F$.

\section{Definição 2.6.}

A relação de satisfatibilidade $(\models)$ associa uma fórmula a uma estrutura e um mundo do seguinte modo:

1) $(\mathrm{M}, w) \vDash p$ com $p \in \Phi$ se, e só se, $\pi_{w}(p)=\mathrm{T}$,

2) $(\mathrm{M}, w) \models \neg \varphi$ se, e só se, $(\mathrm{M}, w) \not \models \varphi$,

\footnotetext{
${ }^{7}$ Nesse caso, são relações de equivalência (omitimos os laços).

${ }^{8}$ Segundo parágrafo da seção 2.2 .
} 
3) $(\mathrm{M}, w) \models \varphi \rightarrow \psi$ se, e só $s e,(\mathrm{M}, w) \not \models \varphi$ ou $(\mathrm{M}, w) \models \psi$,

4) $(\mathrm{M}, w) \vDash K_{i} \varphi$ se, e só se, $(\mathrm{M}, t) \models \varphi$ para todo $t$ tal que $(w, t) \in \mathcal{K}_{i}$.

\section{Observação 2.1.}

Dizemos que uma fórmula $\varphi$ é satisfeita em $(\mathrm{M}, w)$ se e só se $(\mathrm{M}, w) \vDash \varphi$.

As três primeiras cláusulas da definição em 2.6 são análogas às da lógica clássica. A última cláusula formaliza a idéia de que: um agente $i$ conhece $\varphi$ em $w$, numa estrutura $\mathrm{M}$, exatamente se $\varphi$ é verdadeiro em todos os mundos de $\mathrm{M}$ que $i$ considera acessivel a partir de $w$. Graficamente:
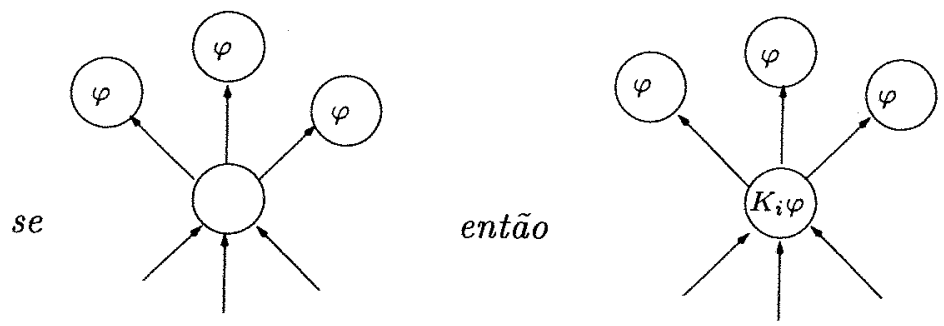

Figura 2.3: Um mundo onde $\varphi$ é conhecido por $i$.

Para caracterizar as propriedades do conhecimento defimos o conceito de fórmulas válidas em relação a uma estrutura $\mathrm{M}$ e fórmulas válidas em relação a uma classe $\mathcal{M}_{n}$ de estruturas.

\section{Definição 2.7 (Satisfatibilidade e Validade).}

Fixado $\Phi$ e $|\Delta|=n$, denotamos por $\mathcal{M}_{n}$ a classe de todas as estruturas de Kripke dos $n$ agentes sobre $\Phi$ sem nenhuma restrição sobre as relações $\mathcal{K}_{i}(i \in \Delta)$. Uma fórmula $\varphi$ é dita ser satisfatível em $\mathrm{M}$ se $(\mathrm{M}, w) \models \varphi$, para algum mundo $w$ em $W$ de $\mathrm{M}$. Uma fórmula $\varphi$ é satisfativel em relação a uma classe $\mathcal{M}_{n}$ se $\varphi$ é satisfativel em alguma estrutura $\mathrm{M}$ de $\mathcal{M}_{n}\left(\left(\mathcal{M}_{n}, \mathrm{M}\right) \models \varphi\right)$.

Dizemos que $\varphi$ é válida em relação a uma estrutura $\mathrm{M}(\mathrm{M} \models \varphi)$, se $(\mathrm{M}, w) \vDash \varphi$, para todo $w(w \in W)$. A validade de uma fórmula $\varphi \mathrm{em}$ relação a uma classe $\mathcal{M}_{n}\left(\mathcal{M}_{n} \vDash \varphi\right)$, se $\varphi$ for válida em todas as estruturas $\mathrm{M}$ de $\mathcal{M}_{n}$.

Note que um molde é um conjunto específico de estruturas de Kripke de $\mathcal{M}_{n}$, esta característica se mostra útil para tratar o problema da validade (vide seção 5.1).

Deixamos, no início desta seção, uma questão pendente. Explicitamos através de um exemplo o segundo motivo pelo qual escolhemos a opção (ii) para a função de valoração. 


\section{Exemplo 2.2.}

Considere como parte do domínio da bivaloração $\pi_{w}$ fórmulas do tipo $K_{i} p(p \in \Phi)$, tal que $\pi_{w}(p)=\mathrm{T}$ e $\pi_{w}\left(K_{i} p\right)=\perp$. A partir de um frame $F$, tome $w$ e uma estrutura $\mathrm{M}=\left\langle W, \pi_{W}, \mathcal{K}_{1}, \ldots, \mathcal{K}_{n}\right\rangle$, tal que para todo i em $\Delta$ temos $(w, w) \in \mathcal{K}_{i}$ e para todo $t$ em $W$, se $(w, t) \in \mathcal{K}_{i}$ então $w=t$. Então, $(\mathrm{M}, w) \vDash K_{i}$ p, para todo $i$, o que contradiz a valoração inicial de $\pi_{w}$.

A seguir damos exemplos da caracterização do conhecimento entre agentes. O primeiro considera a situação do "muddy children puzzle" (Exemplo 2.1). O segundo mostra que um agente conhece uma afirmação contraditória e o terceiro dá a noção de não validade.

\section{Exemplo 2.3.}

Considere a situaçâo do "muddy children puzzle", tal que M é uma estrutura de Kripke, para $n=3$. Verificamos que no mundo $(1,0,1)$ a criança 1 sabe que a criança 2 não está com a testa suja, isto é, $(\mathrm{M},(1,0,1)) \models K_{1}\left(\neg p_{2}\right)$. Por outro lado, a criança 1 não sabe se ela está com a testa suja, ou seja, $(\mathrm{M},(1,0,1)) \models \neg K_{1} p_{1}$ Ainda, um fato que toda criança conhece é se a testa de outra criança está ou não suja $\left(\mathrm{M} \models p_{i} \rightarrow K_{j} p_{i}\right)$, para $j \neq i$.

\section{Exemplo 2.4.}

Seja $\Phi=\{p\}, \Delta=\{1,2\}$ e $W=\{s, t, u\}$ com $\pi_{s}(p)=\pi_{t}(p)=\top$ e $\pi_{u}(p)=\perp$, e tal que $\mathcal{K}_{1}=\{(s, t)\}$ e $\mathcal{K}_{2}=\{(s, u),(u, u)\}$. Verifica-se $q u e(\mathrm{M}, t) \vDash K_{2}(p \wedge \neg p) e(\mathrm{M}, t) \models K_{1} p \wedge K_{2}(\neg p) \wedge K_{1} K_{2}(p \wedge \neg p)$. Uma representação pictórica da situação descrita é dada a seguir:

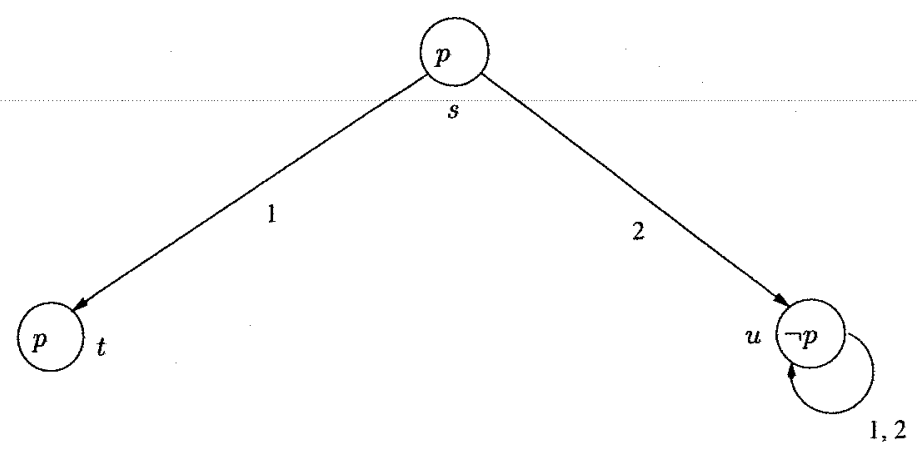

Figura 2.4: Exemplo onde um agente conhece $(p \wedge \neg p)$

\section{Exemplo 2.5.}

Considere $\Phi=\{p\}, \Delta=\{1\}, W=\{s, t\}, \pi_{s}(p)=T$ e $\pi_{t}(p)=\perp, e$ $\mathcal{K}_{1}=\{(s, t)\}$. A representação pictórica correspondente é dada por: 


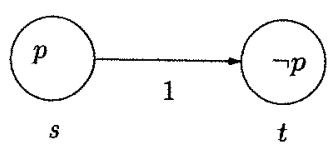

Figura 2.5: Exemplo onde $\varphi \rightarrow K_{1} \varphi$ não é válida.

No exemplo acima $\varphi \rightarrow K_{1} \varphi$ não é verdadeira em $s$. Se $\mathrm{M}=\left\langle W, \pi_{W}, \mathcal{K}_{1}\right\rangle$, então $(\mathrm{M}, s) \not \models \rightarrow K_{1} \varphi$. Portanto $\varphi \rightarrow K_{1} \varphi$ não é válida na classe $\mathcal{M}_{1}$, ou seja, $\varphi \rightarrow K_{i} \varphi$ não é uma fórmula válida. É razoável, pois o fato de $\varphi$ ser verdadeiro não leva ao conhecimento de $\varphi$. O teorema a seguir caracteriza algumas das propriedades de $\models$.

\section{Teorema 2.4 .}

Para quaisquer fórmulas $\varphi, \psi \in \mathrm{K}_{n}(\Phi)$ e qualquer $\mathrm{M}$ de $\mathcal{M}_{n}$ temos:

i) se $\varphi$ for uma instância de tautologia de $\mathrm{CP}$, então $\mathrm{M} \models \varphi$,

ii) se $\mathrm{M} \vDash \varphi$ e $\mathrm{M} \vDash \varphi \rightarrow \psi$, então $\mathrm{M} \vDash \psi$,

iii) $\mathrm{M} \models K_{i}(\varphi \rightarrow \psi) \rightarrow\left(K_{i} \varphi \rightarrow K_{i} \psi\right)$, para qualquer $i, i \in \Delta$,

iv) se $\mathrm{M} \models \varphi$ então $\mathrm{M} \models K_{i} \varphi$, para qualquer $i, i \in \Delta$.

\section{Demonstração.}

Seja $M\left(M \in \mathcal{M}_{n}\right)$ a estrutura de Kripke dada por $\left\langle W, \pi_{W}, \mathcal{K}_{1}, \ldots, \mathcal{K}_{n}\right\rangle$. Com $\pi_{W}$ o conjunto de todas as interpretaçôes modais referentes aos mundos em $W$ e $\mathcal{K}_{i}$ relações sobre $W$. As demonstrações que se seguem independem das propriedades de $\mathcal{K}_{i}$.

i) Da definição de $\pi_{w}$, qualquer fórmula $\varphi$ que é instância de tautologia de CP tem-se que $\pi_{w}(\varphi)=T$, para todo $(w \in W)$. Portanto, $M \models \varphi$.

ii) De $\mathrm{M} \vDash \varphi \rightarrow \psi$ temos que $(\mathrm{M}, w) \vDash \varphi \rightarrow \psi$, isto é, $(\mathrm{M}, w) \not \varphi$ ou $(\mathrm{M}, w) \vDash \psi$, para todo $w$ de $W$. Porém, de $\mathrm{M} \vDash \varphi$ temos que $(\mathrm{M}, w) \models \varphi$, para todo $w$ de $W$. Portanto, $(\mathrm{M}, w) \vDash \psi$, para todo $w$ de $W$ e $M \models \psi$.

iii) Suponha que $\mathrm{M} \not \models K_{i}(\varphi \rightarrow \psi) \rightarrow\left(K_{i} \varphi \rightarrow K_{i} \psi\right)$, isto é, existe $s$ em $\mathrm{M}$ tal que $(\mathrm{M}, s) \not \models K_{i}(\varphi \rightarrow \psi) \rightarrow\left(K_{i} \varphi \rightarrow K_{i} \psi\right)$. Pela definição, temos que $(\mathrm{M}, s) \models K_{i}(\varphi \rightarrow \psi)$ e $(\mathrm{M}, s) \not \models K_{i} \varphi \rightarrow K_{i} \psi$, ou seja, $(\mathrm{M}, s) \vDash K_{i}(\varphi \rightarrow \psi),(\mathrm{M}, s) \vDash K_{i} \varphi$ e $(\mathrm{M}, s) \not \models K_{i} \psi$. Então, para todo $t$ em $\mathrm{M}$ tal que $(s, t) \in \mathcal{K}_{i}$ temos $(\mathrm{M}, t) \models \varphi \rightarrow \psi$ e $(\mathrm{M}, t) \models \varphi$, e de modo análogo ao item anterior $(\mathrm{M}, t) \models \psi$, para todo $t$ tal que 
$(s, t) \in \mathcal{K}_{i}$. Ainda, de $(\mathrm{M}, s) \not \models K_{i} \psi$ tem-se que existe $t^{\prime}$ em $\mathrm{M}$ tal que $\left(s, t^{\prime}\right) \in \mathcal{K}_{i}$ e $\left(\mathrm{M}, t^{\prime}\right) \not \neq \psi$, que contradiz o fato anterior. Portanto, $\mathrm{M} \vDash K_{i}(\varphi \rightarrow \psi) \rightarrow\left(K_{i} \varphi \rightarrow K_{i} \psi\right)$, para qualquer $i, i \in \Delta$.

iv) De $\mathrm{M} \models \varphi$ temos $(\mathrm{M}, s) \models \varphi$ para todo $s$ em M. Em particular, se fixarmos um $w$, então para todo $s$ em $\mathrm{M}$ tal que $(w, s) \in \mathcal{K}_{i}$ temos que $(\mathrm{M}, s) \models \varphi$ e $(\mathrm{M}, w) \vDash K_{i} \varphi$. Como podemos fixar qualquer $w$ em $\mathrm{M}$, $\mathrm{M} \models K_{i} \varphi$.

\section{Observação 2.2 .}

Para quaisquer fórmulas $\varphi, \psi \in \mathrm{K}_{n}(\Phi)$, e qualquer $\mathrm{M}$ de $\mathcal{M}_{n}$ temos:

i) $\mathrm{M} \vDash\left(K_{i} \varphi \wedge K_{i}(\varphi \rightarrow \psi)\right) \rightarrow K_{i} \psi$, para qualquer $i, i \in \Delta$,

ii) $(\mathrm{M}, w) \vDash \varphi \wedge \psi$ se, e somente se, $(\mathrm{M}, w) \vDash \varphi e(\mathrm{M}, w) \vDash \psi$,

iii) $(\mathrm{M}, w) \vDash \varphi \vee \psi$ se, e somente se, $(\mathrm{M}, w) \vDash \varphi$ ou $(\mathrm{M}, w) \vDash \psi$.

A demonstraçấo de (ii) é análoga à do item (iii) do Teorema 2.4. Para a demonstração de (ii) e (iii) basta observar que $\varphi \wedge \psi$ e $\varphi \vee \psi$ são abreviaçôes de $\neg(\varphi \rightarrow \neg \psi) e \neg \varphi \rightarrow \psi$, respectivamente.

A próxima seção apresenta um estudo da relação entre as noções de dedução, consistência, satisfatibilidade e validade.

\subsection{Inferência, Correção e Completude em $\mathrm{K}_{n}$}

Iniciamos esta seção com um comentário sobre regras de inferência e a preservação da verdade pela aplicação dessas regras. Como vimos na seção 1.4 , o conceito de verdade se materializa através das relações de satisfatibilidade e validade que em nosso caso baseiam-se em estruturas relacionais. As noções de satisfatibilidade e validade extendem-se às classes de todas as estruturas de Kripke. Dessa forma, a noção de verdade também se vê modificada ("ampliada"). Uma vez que regras de inferência preservam a verdade, quais os tipos de verdades que estão sendo preservadas pela aplicação de tais regras?

Intuitivamente, dentre as muitas possibilidades podemos caracterizar as regras de inferência do seguinte modo (cf. Fagin [23]):

\section{Observação 2.3 (Regras de Inferência).}

i) regras que preservam a verdade em um mundo, isto é, para decidir se uma dada fórmula é verdadeira num mundo específico de uma particular estrutura (são as chamadas regras que preservam a verdade), 
ii) regras que se aplicam sobre estruturas, isto é, para decidir se uma fórmula é verdadeira em todos os mundos de uma determinada estrutura,

iii) regras sobre frames, ou seja, para decidir se uma fórmula é verdadeira em qualquer mundo num conjunto específico de estruturas,

iv) regras em que nada está fixado, ou seja, para decidir se uma fórmula é verdadeira em todos os mundos de qualquer estrutura em qualquer conjunto de estruturas (são chamadas de regras que preservam a validade).

Em Fagin ([23]), tais regras são vistas como relações sobre fórmulas e são ordenadas por $\subseteq$, por exemplo, em $K_{n}$ o contido é estrito (C). Já em $S 5_{n}$ as inferências sobre frames e as regras que preservam a validade são iguais, isto é, geram o mesmo conjunto de fórmulas.

Nosso estudo baseia nas regras $M P$ que preserva a verdade e $G M$ que, como comentamos no final da seção 1.4, não preserva a verdade como é, porém preserva a validade.

Estamos interessados em preservar a validade sobre classes de estruturas, nesse sentido os teoremas 2.5 e 2.7 nos dão a dimensão exata da relação entre os elementos sintáticos de $\mathrm{K}_{n}$, as regras de inferência e a semântica, para melhor compreensão fazemos uso das seguintes definições.

\section{Definição 2.8 (Correto e Completo).}

Dizemos que um sistema é correto em relação a classe $\mathcal{M}_{n}$, se todo teorema desse sistema for válido em relação a $\mathcal{M}_{n}$, e dizemos que tal sistema é completo em relação a $\mathcal{M}_{n}$ se toda fórmula válida em relação a $\mathcal{M}_{n}$ é teorema nesse sistema.

O teorema a seguir mostra que todo teorema de $K_{n}$ é válido em relação a $\mathcal{M}_{n}$, isto é, $\mathrm{K}_{n}$ é correto em relação a $\mathcal{M}_{n}$.

\section{Teorema 2.5 (Correção).}

$\mathrm{K}_{\mathrm{n}}$ é correto em relaçấo a $\mathcal{M}_{n}$.

Demonstração. [Halpern [36]]

Desejamos mostrar que se $\vdash_{K_{n}} \varphi$, então $\mathcal{M}_{n} \vDash \varphi$. Supondo $\vdash_{K_{n}} \varphi$, a demonstração é feita por indução no número de passos da dedução de $\varphi$ em $K_{n}$. Para a base da indução, supomos que $\vdash_{K_{n}} \varphi$ possui uma única fórmula e nesse caso $\varphi$ é axioma de $K_{n}$.

- se $\varphi$ é instância de tautologia de $C P$, pelo item $(i)$ do Teorema 2.4 $\mathrm{M} \models \varphi$, como vale para qualquer estrutura de $\mathcal{M}_{n}$, temos $\mathcal{M}_{n} \models \varphi$, 
- se $\varphi$ é da forma $K_{i}(\psi \rightarrow \chi) \rightarrow\left(K_{i} \psi \rightarrow K_{i} \chi\right)$, então $\mathcal{M}_{n} \models \varphi$ pelo item (iii) do teorema 2.4 .

Para o passo indutivo, supomos que para qualquer $\varphi$ em $\mathrm{K}_{n}$ se $\vdash_{K_{n}} \varphi$ contém $j$ ou menos passos $(j \geq 1)$, então $\mathcal{M}_{n} \models \varphi$. Seja $\varphi$ tal que $\vdash_{\kappa_{n}} \varphi$ em $j+1$ passos, isto é, $\varphi_{j+1}=\varphi$, então ou $\varphi$ é instância de axioma de $\mathrm{K}_{n}$ e pela base $\mathcal{M}_{n} \vDash \varphi$; ou $\varphi$ segue de $M P$ ou de GM. Caso $\varphi$ decorra de $M P$, pelo item (ii) do Teorema $2.4 \mathcal{M}_{n} \vDash \varphi$, senão se $\varphi$ é obtida por $G M$, então pelo item (iv) do Teorema $2.4 \mathcal{M}_{n} \vDash \varphi$. Portanto, $\mathrm{K}_{n}$ é correto em relação a $\mathcal{M}_{n}$.

O teorema a seguir nos fornece uma estrutura de Kripke que satisfaz fórmulas $\mathrm{K}_{n}$-consistentes. A construção dos conjuntos maximais consistentes segue o método devido a Makinson (1966).

\section{Teorema 2.6 (Estruturas Canônicas).}

Toda fórmula $\kappa_{n}$-consistente é satisfatível em alguma estrutura de $\mathcal{M}_{n}$.

Demonstração. [Halpern [36]]

Devemos exibir uma estrutura em $\mathcal{M}_{n}$ e um mundo nessa estrutura que satisfaça toda fórmula $K_{\pi}$ consistente. Chamamos essa estrutura de $M_{\text {can }}$ com $\mathrm{M}_{\mathrm{can}}=\left\langle W, \pi_{W}, \mathcal{K}_{1}, \ldots, \mathcal{K}_{n}\right\rangle$ e a definimos como segue:

$$
\begin{aligned}
& W=\left\{w_{\Gamma} \mid \Gamma \text { é um conjunto maximal consistente }\right\} \\
& \pi_{w_{\Gamma}}(\varphi)= \begin{cases}T & \text { se } \varphi \in \Gamma \\
\perp & \text { se } \varphi \notin \Gamma\end{cases} \\
& \mathcal{K}_{i}=\left\{\left(w_{\Gamma}, w_{\Gamma^{\prime}}\right) \mid \Gamma / K_{i} \subseteq \Gamma^{\prime}\right\}, \text { com } \Gamma / K_{i}=\left\{\varphi \mid K_{i} \varphi \in \Gamma\right\} .
\end{aligned}
$$

Para demonstrar que toda fórmula $\kappa_{n}$-consistente é satisfatível em $M_{\text {can }}$ é suficiente demonstrar que existe um mundo $w_{\Sigma}$ em $\mathrm{M}_{\text {can }}$ tal que:

$$
\left(\mathrm{M}_{\mathrm{can}}, w_{\Sigma}\right) \models \varphi \text { se, e somente se, } \varphi \in \Sigma,
$$

pois pelo lema de Lindenbaun (Prop. 2.3) se $\varphi$ é $\mathrm{K}_{\tilde{n}}$-consistente, então $\varphi$ pertence a algum conjunto $\Sigma$ maximal consistente de $K_{n}(\Phi)$, e por $(\dagger)$ segue que $\left(\mathrm{M}_{\mathrm{can}}, w_{\Sigma}\right) \vDash \varphi$ e $\varphi$ é satisfatível em alguma estrutura de $\mathcal{M}_{n}$.

A demonstração da afirmação em $(\dagger)$ segue por indução no número de conectivos que ocorrem em $\varphi$. Seja $\Sigma$ conjunto maximal consistente em $\mathrm{K}_{n}(\Phi)$ :

- se $\varphi \in \Phi$, então pela definição de satisfatibilidade (Def. 2.6) $\left(\mathrm{M}_{\mathrm{can}}, w_{\Sigma}\right) \models \varphi$ se, e somente se, $\pi_{w_{\Sigma}}(\varphi)=T$ se, e só, se $\varphi \in \Sigma$,

- se $\varphi$ é da forma $\neg \psi$, então $\varphi \in \Sigma$ se, e só se, $\psi \notin \Sigma$ (pelo item (ii) da Proposição 2.3). Pela hipótese de indução, como $\psi$ possui menos 
conectivos, então pela definição em 2.6 se, e somente se, $\left(\mathrm{M}_{\text {can }}, w\right) \not \psi$ que equivale a $\left(\mathrm{M}_{\mathrm{can}}, w\right) \models \varphi$,

- se $\varphi$ é da forma $\psi \rightarrow \chi$. Temos que $\psi \rightarrow \chi \in \Sigma$ se, e somente se, $\psi \notin \Sigma$ ou $\chi \in \Sigma$ (pelos itens (iii) e (ii) da Proposição 2.3). Como $\psi$ e $\chi$ possuem menos conectivos que $\varphi$, pela hipótese de indução se, e somente se, $\left(\mathrm{M}_{\mathrm{can}}, w_{\Sigma}\right) \not \models \psi$ ou $\left(\mathrm{M}_{\mathrm{can}}, w_{\Sigma}\right) \models \chi$, que equivale a $\left(\mathrm{M}_{\text {can }}, w_{\Sigma}\right) \models \varphi$,

- se $\varphi$ é da forma $K_{i} \psi$. Primeiramente, vamos mostrar que se $K_{i} \psi \in \Sigma$, então $\left(\mathrm{M}_{\text {can }}, w_{\Sigma}\right) \models \varphi$. Sabemos que $K_{i} \psi \in \Sigma$ se, e somente se, $\psi \in \Sigma / K_{i}$, pela definição de $\Sigma / K_{i}$. Para todo $w_{\Sigma^{\prime}} \mathrm{em}$ $\mathrm{M}_{\text {can }}$ tal que $\left(w_{\Sigma}, w_{\Sigma^{\prime}}\right) \in \mathcal{K}_{i}$, tem-se que $\psi \in \Sigma^{\prime}$, pois $\Sigma / K_{i} \subseteq \Sigma^{\prime}$, então $\pi_{w_{\Sigma^{\prime}}}(\psi)=\mathrm{T}$ e $\left(\mathrm{M}_{\mathrm{can}}, w_{\Sigma^{\prime}}\right) \models \psi$. Pela hipótese de indução, $\left(\mathrm{M}_{\text {can }}, w_{\Sigma}\right) \models \varphi$.

Por outro lado, se $\left(\mathrm{M}_{\text {can }}, w_{\Sigma}\right) \models \varphi$, então $\Sigma / K_{i} \cup\{\neg \psi\}$ é inconsistente. De fato, suponha $\Sigma / K_{i} \cup\{\neg \psi\}$ consistente, então pelo lema de Lindenbaun (Prop. 2.3) existe um conjunto maximal consistente $\Sigma^{\prime}$ tal que $\Sigma / K_{i} \cup\{\neg \psi\} \subseteq \Sigma^{\prime}$, ou seja, $\left(w_{\Sigma}, w_{\Sigma^{\prime}}\right) \in \mathcal{K}_{i}$. Como $\left(\mathrm{M}_{\text {can }}, w_{\Sigma}\right) \models K_{i} \psi$ e $\left(w_{\Sigma}, w_{\Sigma^{\prime}}\right) \in \mathcal{K}_{i}$, então $\left(\mathrm{M}_{\text {can }}, w_{\Sigma^{\prime}}\right) \models \psi$, pela hipótese de indução $\psi \in \Sigma^{\prime}$. Mas $\neg \psi \in \Sigma^{\prime}$, contradição.

Agora, vamos mostrar que se $\Sigma / K_{i} \cup\{\neg \psi\}$ é inconsistente, então $K_{i} \psi \in \Sigma$. Da suposição tem-se que existe um subconjunto finito $\left\{\varphi_{1}, \ldots, \varphi_{j}, \neg \psi\right\}$ de $\Sigma / K_{i} \cup\{\neg \psi\}$ tal que $\vdash_{\kappa_{n}} \neg\left(\varphi_{1} \wedge \cdots \wedge \varphi_{j} \wedge \neg \psi\right) . \quad \operatorname{Como}_{\kappa_{n}} \neg\left(\varphi_{1} \wedge \cdots \wedge \varphi_{j} \wedge \neg \psi\right)$ abrevia $\vdash_{k_{n}} \varphi_{1} \rightarrow\left(\varphi_{2} \rightarrow\left(\varphi_{3} \rightarrow \cdots \rightarrow\left(\varphi_{j} \rightarrow \psi\right) \cdots\right)\right)$, de $G M$ e $M P$ temos $\vdash_{\kappa_{n}} K_{i} \varphi_{1} \rightarrow K_{i}\left(\varphi_{2} \rightarrow\left(\varphi_{3} \rightarrow \cdots \rightarrow\left(\varphi_{j} \rightarrow \psi\right) \cdots\right)\right)$. Do axioma $K$ e pelo silogismo hipotético (observação em 1.2) temos que: $\quad \vdash_{\kappa_{n}} K_{i} \varphi_{1} \rightarrow\left(K_{i} \varphi_{2} \rightarrow K_{i}\left(\varphi_{3} \rightarrow \cdots \rightarrow\left(\varphi_{j} \rightarrow \psi\right) \cdots\right)\right)$, então repetindo o processo $j-1$ vezes obtemos $\vdash_{K_{n}} K_{i} \varphi_{1} \rightarrow\left(K_{i} \varphi_{2} \rightarrow\left(K_{i} \varphi_{3} \rightarrow \cdots \rightarrow\left(K_{i} \varphi_{j} \rightarrow K_{i} \psi\right) \cdots\right)\right)$.

Logo, $\quad \vdash_{K_{n}} \neg\left(K_{i} \varphi_{1} \wedge K_{i} \varphi_{2} \wedge \cdots \wedge K_{i} \varphi_{j} \wedge \neg K_{i} \psi\right)$, ou seja, $\left\{K_{i} \varphi_{1}, K_{i} \varphi_{2}, \ldots, K_{i} \varphi_{j}, \neg K_{i} \psi\right\} \quad$ é inconsistenter $\left\{K_{i} \varphi_{1}, K_{i} \varphi_{2}, \ldots, K_{i} \varphi_{j}\right\} \subseteq \Sigma$, então $K_{i} \psi \in \Sigma$.

Então, $\left(\mathrm{M}_{\mathrm{can}}, w_{\Sigma}\right) \models \varphi$ se, e só se, $\varphi \in \Sigma$, para $\Sigma$ maximal consistente.

\section{Teorema 2.7 (Completude).}

$\mathrm{K}_{\mathrm{n}}$ é completo em relação a $\mathcal{M}_{n}$.

Demonstração. [Halpern [36]]

Toda fórmula válida em relação a $\mathcal{M}_{n}$ é $\kappa_{n}$-dedutível. De fato, seja $\varphi$ 
válida em relação a $\mathcal{M}_{n}$ e suponha que $\varphi$ não é $\kappa_{n}$-dedutível, isto é, $\not \neg \neg \neg \varphi$ em $K_{n}$ (vide observação em 1.2), então $\neg \varphi$ é $K_{n}$ consistente. E, pelo teorema anterior $\neg \varphi$ é satisfatível em alguma estrutura de $\mathcal{M}_{n}$ contradizendo a validade de $\varphi$. Portanto, $K_{n}$ é completo em relação a $\mathcal{M}_{n}$.

Os teoremas 2.5 e 2.7 mostram que as propriedades operacionais de $\vdash$ descrevem as propriedades de $\models$ e vice-versa, ou seja, são equivalentes em $\mathrm{K}_{n}$, portanto podemos falar de consistência e provabilidade em termos de satisfatibilidade e validade. A seguir apresentamos outros sistemas de conhecimento, obtidos a partir de $\mathrm{K}_{n}$, e verificamos a correção e completude desses sistemas. 


\section{Extensões do Sistema $\mathrm{K}_{n}$}

Outras axiomáticas modais podem ser construídas a partir de $K_{n}$. Dependendo das propriedades epistêmicas que desejamos atribuir aos agentes podemos escolher diferentes sistemas de conhecimento. Neste capítulo, apresentamos os sistemas $\mathrm{T}_{n}, \mathrm{~S} 4_{n}, \mathrm{~S} 5_{n}$ e $\mathrm{KD} 45_{n}$ obtidos a partir dos sistemas modais T, S4, S5 e KD45, respectivamente. Primeiramente, comentamos os axiomas inclusos à $K_{n}$ e classificamos as diversas extensões obtidas. $\mathrm{Na}$ seção 3.2 mostramos que os sistemas são corretos e completos em relação às respectivas semânticas. A seguir analisamos algumas propriedades das estruturas de Kripke a partir da noção de modelo. Finalmente, estudamos os resultados de correção e completude em estruturas finitas.

\subsection{Os sistemas: $\mathrm{T}_{n}, \mathrm{~S} 4_{n}, \mathrm{~S} 5_{n}$ e $\mathrm{KD} 45_{n}$}

$\mathrm{O}$ sistema $\mathrm{T}_{n}$ é obtido acrescentando à $\mathrm{K}_{n}$ o esquema de axioma $K_{i} \varphi \rightarrow \varphi$, chamado de $T$. Esse sistema é usado para expressar o conhecimento que é suposto perfeito, isto é, o axioma diz que: se $\varphi$ é conhecido, então $\varphi$ é verdadeiro, isto é, somente fatos verdadeiros são conhecidos.

Se desejarmos analisar o conhecimento não tão perfeito podemos optar pelo esquema $D$ dado por $K_{i} \varphi \rightarrow \neg K_{i}(\neg \varphi)$, que é uma versão fraca do axioma anterior ( $c f$. Proposição 3.1). O axioma $D$ pode ser lido como: é falso que se conheçam fatos contraditórios.

Outro sistema interessante é o $S 4_{n}$ formado pelo sistema $T_{n}$ mais o axioma 4: $K_{i} \varphi \rightarrow K_{i}\left(K_{i} \varphi\right)$ que pode ser parafraseado como: sei o que sei. Inicialmente, $S 4_{n}$ era composto pelo axioma $B: \varphi \rightarrow K_{i}\left(\neg K_{i}(\neg \varphi)\right)$ e foi construído para formalizar o intuicionismo de Brouwer, porém mostrou-se 
falho. Gödel mostrou que o intuicionismo pode ser formalizado por $\mathrm{S}_{n}$ substituindo-se $B$ por 4 ( $c f$. Troelstra [81, sec. 9.1 e 9.2]). $O$ axioma $B$ também não se mostrou adequado para a formalização de sistemas envolvendo conhecimento e atualmente é utilizado para a formalização da lógica quântica (cf. Marchal [54, págs. 15-23]).

O sistema $\mathrm{S} 5_{n}$ é obtido adicionando o axioma $5: \neg K_{i} \varphi \rightarrow K_{i} \neg K_{i} \varphi$ a $\mathrm{T}_{n}$, e que pode ser parafraseado por: sei o que não conheço, é uma espécie de introspecção negativa.

\section{Observação 3.1.}

Em Halpern e Moses [36], o sistema $\mathrm{KD}_{4} 5_{n}$ é obtido de $\mathrm{K}_{4} 5_{n}$ com a inclusão do axioma $\neg K_{i}(\text { false })^{1}$, onde false indica uma fórmula contraditória do tipo $\neg(\varphi \rightarrow \varphi)$ (vide Proposição 1.1). A preferência pelo esquema de axioma $D$ ao $\neg K_{i}$ (false) é apenas por questão estética (veja que false não faz parte da linguagem de $\left.\mathrm{KD} 45_{n}\right)$, pois são equivalentes: $K_{i} \varphi \rightarrow \neg K_{i}(\neg \varphi)$ pode ser escrito como $\neg\left(K_{i} \varphi \wedge K_{i}(\neg \varphi)\right)$, da definição de $\wedge$, que equivale $\grave{a}$ $\neg K_{i}(\varphi \wedge \neg \varphi)$ (notas em 1.2).

De fato, podemos estabelecer uma ordem parcial entre os sistemas apresentados por meio da relação $\vdash$ (de prova).

\section{Proposição 3.1.}

Dados dois sistemas $\mathrm{S}_{1}$ e $\mathrm{S}_{2}$ escrevemos $\mathrm{S}_{1} \preceq \mathrm{S}_{2}$ se $\vdash_{\mathrm{S}_{2}} \varphi$, então $\vdash_{\mathrm{S}_{1}} \varphi$. Com isso podemos afirmar que $\mathrm{KD} \preceq \mathrm{T} e \mathrm{~S} 4 \preceq \mathrm{S} 5$.

\section{Demonstração. (Popkorn [66])}

Para verificar que S4 $\preceq$ S5 basta mostrar que $5 \rightarrow 4$ :

1. $\vdash_{\mathrm{Ss}_{n}} \neg K_{i} \neg K_{i} \varphi \rightarrow K_{i} \neg K_{i} \neg K_{i} \varphi$

2. $\quad \vdash_{S 5_{n}} K_{i} \neg K_{i} \varphi \rightarrow \neg K_{i} \varphi$

3. $\vdash_{S 5_{n}}\left(K_{i} \neg K_{i} \varphi \rightarrow \neg K_{i} \varphi\right) \rightarrow\left(K_{i} \varphi \rightarrow \neg K_{i} \neg K_{i} \varphi\right)$

4. $\vdash_{55_{n}} K_{i} \varphi \rightarrow \neg K_{i} \neg K_{i} \varphi$

$5 \quad \vdash_{55_{n}} K_{i} \varphi \rightarrow K_{i} \neg K_{i} \neg K_{i} \varphi$

6. $\quad \vdash_{55_{n}} \neg K_{i} \neg(\neg \varphi) \rightarrow K_{i} \neg K_{i} \neg(\neg \varphi)$

7. $\quad \vdash_{55_{n}}\left(\neg K_{i} \varphi \rightarrow K_{i} \neg K_{i} \varphi\right) \rightarrow\left(\neg\left(K_{i} \neg K_{i} \varphi\right) \rightarrow \neg\left(\neg K_{i} \varphi\right)\right)$

8. $\quad \vdash_{55_{n}} \neg\left(K_{i} \neg K_{i} \varphi\right) \rightarrow K_{i} \varphi$

9. $\quad \vdash_{55_{n}} K_{i}\left(\neg K_{i} \neg K_{i} \varphi \rightarrow K_{i} \varphi\right)$

10. $\vdash_{55_{n}} K_{i}\left(\neg K_{i} \neg K_{i} \varphi \rightarrow K_{i} \varphi\right) \rightarrow\left(K_{i} \neg K_{i} \neg K_{i} \varphi \rightarrow K_{i} K_{i} \varphi\right)$

11. $\vdash_{\mathrm{S5n}} K_{i} \neg K_{i} \neg K_{i} \varphi \rightarrow K_{i} K_{i} \varphi$

12. $\vdash_{\mathrm{s5n}_{n}} K_{i} \varphi \rightarrow K_{i} K_{i} \varphi$ 5 $T$ Teor. de $\mathrm{K}_{n}$ $M P$ SH 3, 1 5 Teor. de $\mathrm{K}_{n}$ $M P 4,5$ $G M$ $K$ $M P$ $S H 5,11$

\footnotetext{
${ }^{1}$ false pode ser substituido por $\perp$.
} 
A demonstração de $T \rightarrow D$ é como segue:

1. $\vdash_{\mathrm{T}_{n}} K_{i} \neg \varphi \rightarrow \neg \varphi$

2. $\vdash_{\mathrm{T}_{n}}\left(K_{i} \neg \varphi \rightarrow \neg \varphi\right) \rightarrow\left(\varphi \rightarrow \neg K_{i} \neg \varphi\right)$

3. $\vdash_{T_{n}} \varphi \rightarrow \neg K_{i} \neg \varphi$

$T$

4. $\vdash_{T_{n}} K_{i} \varphi \rightarrow \varphi$

Teor. de $\mathrm{K}_{n}$

5. $\vdash_{T_{n}}\left(K_{i \varphi} \rightarrow \varphi\right) \rightarrow\left(\left(\varphi \rightarrow \neg K_{i} \neg \varphi\right) \rightarrow\left(K_{i \varphi} \rightarrow \neg K_{i} \neg \varphi\right)\right) T$

$M P$

6. $\vdash_{\mathrm{T}_{n}}\left(\varphi \rightarrow \neg K_{i} \neg \varphi\right) \rightarrow\left(K_{i} \varphi \rightarrow \neg K_{i} \neg \varphi\right)$

Taut. de $\mathrm{CP}$

7. $\vdash_{\mathrm{T}_{n}} K_{i} \varphi \rightarrow \neg K_{i} \neg \varphi$

$M P$

$M P$

Como conseqüência da proposição temos uma ordenação pela relação $\preceq$ entre os sistemas mencionados, graficamente temos:

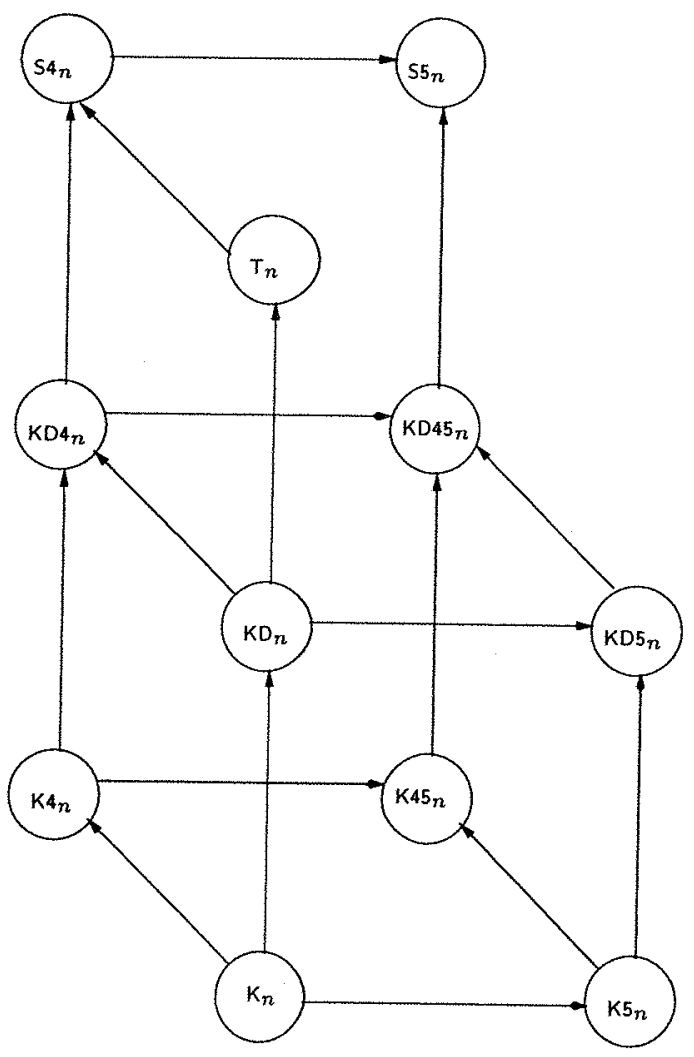

Figura 3.1: Relação entre os sistemas de conhecimento. $K 4_{n}$ é o sistema formado pelos axiomas $K$ e 4 com $n$ agentes, e assim por diante.

A seguir estudamos as semânticas dos sistemas $T_{n}, S 4_{n}, S 5_{n}$ e $K D 45_{n}$, antes porém analisamos algumas propriedades das relações $\mathcal{K}_{i}$. 
Há uma correspondência direta entre fórmulas modais e fórmulas da lógica de primeira ordem, cada um dos axiomas exibe uma propriedade que reflete diretamente nas características das relações de acessibilidade e viceversa $\mathcal{K}_{i}\left(c f . \operatorname{van}\right.$ Benthem [83, pág. 11]). Por exemplo, o axioma $K_{i} \varphi \rightarrow \varphi$ equivale a $\forall i \forall w\left((w, w) \in \mathcal{K}_{i}\right)$, essa correspondência determina:

\section{Observação 3.2 .}

i) um comportamento aos operadores modais semelhante ao do quantificador universal, porém restrito ao conjunto de mundos possiveis do agente $i$, a partir de um mundo $w$ (o mundo onde o agente se encontra) via $\mathcal{K}_{i}$

ii) condiçôes simples que impostas sobre as relações $\mathcal{K}_{i}$ geram estruturas cujas fórmulas válidas nessas estruturas são exatamente as fórmulas dedutíveis, fato primeiramente demonstrado por Kripke.

Algumas características básicas das relações $\mathcal{K}_{i}$.

\section{Definição 3.1.}

Seja $i \in \Delta$. Dizemos que $\mathcal{K}_{i}$ é euclideano, se $(w, s) \in \mathcal{K}_{i}$ e $(w, t) \in \mathcal{K}_{i}$, então $(s, t) \in \mathcal{K}_{i}$, para todo $w, s, t \in W$. $\mathcal{K}_{i}$ é serial, se para todo $w \in W$ existe $s \in W$ tal que $(w, s) \in \mathcal{K}_{i}$.

Segue de modo imediato que:

\section{Proposição 3.2.}

i) se $\mathcal{K}_{i}$ é simétrico e transitivo, então $\mathcal{K}_{i}$ é euclideano,

ii) $\mathcal{K}_{i}$ é simétrico, transitivo e serial se, e só se, $\mathcal{K}_{i}$ é reflexivo e euclideano se, e só se, $\mathcal{K}_{i}$ é relação de equivalência.

\section{Demonstração.}

Para verificar o primeiro item sejam $(w, s)$ e $(w, t)$ elementos de $\mathcal{K}_{i}$. Pela simetria, temos que $(s, w)$ e $(t, w)$ são elementos de $\mathcal{K}_{i}$ e pela transitividade $(s, t) \in \mathcal{K}_{i}$ e $(t, s) \in \mathcal{K}_{i}$, portanto $\mathcal{K}_{i}$ é euclideano. As equivalências do outro item seguem de modo análogo.

A proposição a seguir mostra, do ponto de vista semântico, que algumas propriedades sobre as relações de $\mathcal{K}_{i}$ induzem a respectiva estrutura a satisfazer certas fórmulas.

\section{Proposição 3.3.}

$$
\text { Seja } \mathrm{M}=\left\langle W, \pi_{W}, \mathcal{K}_{1}, \ldots, \mathcal{K}_{n}\right\rangle \text { e } \Delta=\{1,2, \ldots, n\}
$$



i) se as relações $\mathcal{K}_{i}(i \in \Delta)$ são reflexivas, então $M \models K_{i} \varphi \rightarrow \varphi$,
ii) se as relações $\mathcal{K}_{i}$ são transitivas, então $\mathrm{M} \models K_{i} \varphi \rightarrow K_{i} K_{i} \varphi$,
iii) se as relações $\mathcal{K}_{i}$ são simétricas e transitivas, $\mathrm{M} \models \neg K_{i} \varphi \rightarrow K_{i} \neg K_{i} \varphi$,
iv) se as relaçôes $\mathcal{K}_{i}$ são seriais, então $\mathrm{M} \models K_{i} \varphi \rightarrow \neg K_{i} \neg \varphi$.

\section{Demonstração.}

i) Suponha que $\mathrm{M} \not \models K_{i} \varphi \rightarrow \varphi$, então existe $w \operatorname{com}(\mathrm{M}, w) \not \models K_{i} \varphi \rightarrow \varphi$, isto é, $(\mathrm{M}, w) \vDash K_{i} \varphi$ e $(\mathrm{M}, w) \not \varphi \varphi$. Como $\mathcal{K}_{i}$ é reflexiva, então $(w, w) \in \mathcal{K}_{i}$, para todo $w$ em $\mathrm{M}$ e temos uma contradição. Portanto, $\mathrm{M} \vDash K_{i} \varphi \rightarrow \varphi$.

ii) Analogamente, suponha $\mathrm{M} \not \models K_{i} \varphi \rightarrow K_{i} K_{i} \varphi$, isto é, existe $w$ tal que $(\mathrm{M}, w) \not K_{i} \varphi \rightarrow K_{i} K_{i} \varphi, \operatorname{logo}(\mathrm{M}, w) \vDash K_{i} \varphi$ e $(\mathrm{M}, w) \not \models K_{i} K_{i} \varphi$. De $(\mathrm{M}, w) \not \models K_{i} K_{i} \varphi$ existe $s \operatorname{com}(w, s) \in \mathcal{K}_{i} \mathrm{e}(\mathrm{M}, s) \not \models K_{i} \varphi$, que por sua vez implica na existência de $t$ tal que $(s, t) \in \mathcal{K}_{i}$ e $(\mathrm{M}, t) \not \forall \varphi$. Como $\mathcal{K}_{i}$ é transitiva, então $(w, t) \in \mathcal{K}_{i}$ contradizendo $(\mathrm{M}, w) \vDash K_{i} \varphi$.

iii) Novamente, vamos supor que $\mathrm{M} \not \models \neg K_{i} \varphi \rightarrow K_{i} \neg K_{i} \varphi$. Então, existe $w$ tal que $(\mathrm{M}, w) \not \models K_{i} \varphi$ e $(\mathrm{M}, w) \not \models K_{i} \neg K_{i} \varphi$, logo existe $s$ tal que $(w, s) \in \mathcal{K}_{i}$ e $(\mathrm{M}, s) \models K_{i} \varphi$, ou seja, para todo $t$ tal que $(s, t) \in \mathcal{K}_{i}$ tem-se que $(\mathrm{M}, t) \vDash \varphi$. Por outro lado, considere $r$ tal que $(w, r) \in \mathcal{K}_{i}$, pela simetria de $\mathcal{K}_{i}$ temos $(r, w) \in \mathcal{K}_{i}$, da transitividade $(r, s) \in \mathcal{K}_{i}$ e $(s, r) \in \mathcal{K}_{i}$. Assim, não existe $r$ tal que $(w, r) \in \mathcal{K}_{i}$ e satisfaça $(\mathrm{M}, w) \not \models K_{i} \varphi$. Portanto, $\mathrm{M} \models \neg K_{i} \varphi \rightarrow K_{i} \neg K_{i} \varphi$.

iv) Por fim, se $\mathrm{M} \not \models K_{i} \varphi \rightarrow \neg K_{i} \neg \varphi$, então existe $w$ tal que (M, $\left.w\right) \vDash K_{i} \varphi$ e $(\mathrm{M}, w) \models K_{i} \neg \varphi$. Ou seja, para todo $s$ tal que $(w, s) \in \mathcal{K}_{i}$ devemos ter $(\mathrm{M}, s) \models \varphi$ e $(\mathrm{M}, s) \models \neg \varphi$. De $\mathcal{K}_{i}$ ser serial temos a existência de um tal $s$ e uma contradição. Portanto, $\mathrm{M} \models K_{i} \varphi \rightarrow \neg K_{i} \neg \varphi$.

A proposição seguinte mostra as propriedades das relações $\mathcal{K}_{i}$ de $\mathrm{M}_{\text {can }}$ de acordo com os axiomas de conhecimento.

\section{Proposição 3.4.}

Seja $\mathrm{M}_{\text {can }}$ como definida no Teorema 2.6.

i) se $\mathrm{M}_{\mathrm{can}} \models K_{i} \varphi \rightarrow \varphi$, entẫo $\mathcal{K}_{i}$ é reflexiva,

ii) se $\mathrm{M}_{\mathrm{can}} \models K_{i} \varphi \rightarrow K_{i} K_{i} \varphi$, então $\mathcal{K}_{i}$ é transitiva,

iii) se $\mathrm{M}_{\text {can }} \models \neg K_{i} \varphi \rightarrow K_{i} \neg K_{i} \varphi$, então $\mathcal{K}_{i}$ é euclideano, 
iv) se $\mathrm{M}_{\mathrm{can}} \models K_{i} \varphi \rightarrow \neg K_{i} \neg \varphi$, então $\mathcal{K}_{i}$ é serial.

Demonstração. [Halpern [36]]

i) $\mathrm{M}_{\text {can }} \models K_{i} \varphi \rightarrow \varphi$ se, e só se, $\left(\mathrm{M}_{\text {can }}, w_{\Sigma}\right) \models K_{i} \varphi \rightarrow \varphi$, para todo $w_{\Sigma}$ em $\mathrm{M}_{\text {can }}$; que equivale a dizer que se $K_{i} \varphi \in \Sigma$, então $\varphi \in \Sigma$, ou seja, $\Sigma / K_{i} \subset \Sigma$. Portanto, $\left(\mathrm{M}_{\mathrm{can}}, w_{\Sigma}\right) \models K_{i} \varphi \rightarrow \varphi$ se, e só se, $\left(w_{\Sigma}, w_{\Sigma}\right) \in \mathcal{K}_{i}$, para todo $w_{\Sigma}$ em $\mathrm{M}_{\text {can }}$.

ii) $\left(\mathrm{M}_{\text {can }}, w_{\Sigma}\right) \models K_{i} \varphi \rightarrow K_{i} K_{i} \varphi$, isto é, se $K_{i} \varphi \in \Sigma$, então $K_{i} K_{i} \varphi \in \Sigma$ $\left(K_{i} \varphi \in \Sigma / K_{i}\right)$, para todo $w_{\Sigma}$ em $\mathrm{M}_{\text {can }}$. Então, se $K_{i} \varphi \in \Sigma \mathrm{e}$ $\left(w_{\Sigma}, w_{\Sigma^{\prime}}\right) \in \mathcal{K}_{i}$, temos que $K_{i} \varphi \in \Sigma / K_{i}$ e $K_{i} \varphi \in \Sigma^{\prime} ; \operatorname{logo}, \quad\left(w_{\Sigma^{\prime}}, w_{\Sigma^{\prime \prime}}\right) \in$ $\mathcal{K}_{i}$ implica que $\varphi \in \Sigma^{\prime \prime}$ e $\mathcal{K}_{i}$ é transitiva.

iii) Tome $\left(w_{\Sigma}, w_{\Sigma^{\prime}}\right)$ e $\left(w_{\Sigma}, w_{\Sigma^{\prime \prime}}\right)$ elementos em $\mathcal{K}_{i}$ e suponha que $\left(w_{\Sigma^{\prime}}, w_{\Sigma^{\prime \prime}}\right) \notin \mathcal{K}_{i}$, isto é, existe $K_{i} \varphi$ elemento de $\Sigma^{\prime}$ e $\varphi \notin \Sigma^{\prime \prime}$. Logo, se $K_{i} \varphi \in \Sigma^{\prime}$, então $\left(\mathrm{M}_{\text {can }}, w_{\Sigma^{\prime}}\right) \models K_{i} \varphi$ e $\left(\mathrm{M}_{\text {can }}, w_{\Sigma}\right) \models \neg K_{i} \neg K_{i} \varphi$, pois $\left(w_{\Sigma}, w_{\Sigma^{\prime}}\right) \in \mathcal{K}_{i}$ e portanto $\left(\mathrm{M}_{\text {can }}, w_{\Sigma}\right) \vDash K_{i} \varphi$ (pela contrapositiva da hipótese). Então, $\left(\mathrm{M}_{\text {can }}, w_{\Sigma^{\prime \prime}}\right) \models \varphi$, já que $\left(w_{\Sigma}, w_{\Sigma^{\prime \prime}}\right) \in \mathcal{K}_{i}$ e temos uma contradição com o fato de $\varphi \notin \Sigma^{\prime \prime}$. Segue que $\mathcal{K}_{i}$ é euclideano.

iv) De $\mathrm{M}_{\text {can }} \vDash K_{i} \varphi \rightarrow \neg K_{i} \neg \varphi$ temos que se $\left(\mathrm{M}_{\text {can }}, w_{\Sigma}\right) \vDash K_{i} \varphi$, então $\left(\mathrm{M}_{\text {can }}, w_{\Sigma}\right) \models \neg K_{i} \neg \varphi$, para todo $w_{\Sigma}$ de $\mathrm{M}_{\text {can }}$. Suponha que exista $w_{\Sigma}$ tal que para qualquer $w_{\Sigma^{\prime}}$ em $\mathrm{M}_{\mathrm{can}}$ temos $\left(w_{\Sigma}, w_{\Sigma^{\prime}}\right) \notin \mathcal{K}_{i}$. Isto é, existe $K_{i} \varphi$ elemento de $\Sigma$ e $\varphi \notin \Sigma^{\prime}$, logo se $K_{i} \varphi \in \Sigma$, então $\left(\mathrm{M}_{\text {can }}, w_{\Sigma}\right) \vDash K_{i} \varphi$ e $\left(\mathrm{M}_{\text {can }}, w_{\Sigma}\right) \models \neg K_{i} \neg \varphi$, pela hipótese inicial. Portanto, existe $w_{\Sigma^{\prime}}$ tal que $\left(w_{\Sigma}, w_{\Sigma^{\prime}}\right) \notin \mathcal{K}_{i}$ e $\left(\mathrm{M}_{\text {can }}, w_{\Sigma^{\prime}}\right) \models \varphi$, ou seja, $\operatorname{com} \varphi \in \Sigma^{\prime}$. A contradição nos leva a tese.

Pelas proposições 3.3 e 3.4 podemos assumir que cada sistema possui uma estrutura semântica segundo os axiomas escolhidos, sistemas que contém $T$ (resp., 4,5 e $D$ ) como um de seus axiomas forçam uma semântica tal que suas estruturas tenham relações de acessibilidade reflexivas (resp., transitivas, euclideanas e seriais). A teoria da correspondência estuda a correlação existente entre axiomas modais e propriedades das relações de acessibilidades. Está fora de nosso escopo desenvolver tal estudo. As idéias aqui expostas foram extraídas de van Benthem [84, cap. II.4] e Blackburn [2]. Na próxima seção efetivamos as relações entre os conjuntos de teoremas de cada sistema de conhecimento e certas classes de estruturas. 


\subsection{Correção e Completude}

Nesta seção exibimos subclasses $\mathcal{M}_{n}^{r}$ (resp., $\mathcal{M}_{n}^{r t}, \mathcal{M}_{n}^{r s t}, \mathcal{M}_{n}^{\text {elt }}$ ) de $\mathcal{M}_{n}$ obtidas através de certas condições impostas às relações $\mathcal{K}_{i}$. Verificamos que as fórmulas válidas nessas subclasses são exatamente as fórmulas dedutíveis de $T_{n}$ (resp., $S 4_{n}, S 5_{n}$ e $K D 45_{n}$ ). As definições relativas à consistência, correção e completude em $\mathrm{T}_{n}$ (resp., $\mathrm{S} 4_{n}, \mathrm{~S} 5_{n}$ e $\mathrm{KD} 45_{n}$ ) são análogas às de $\mathrm{K}_{n}$. Seguem as adaptações necessárias:

\section{Definição 3.2 .}

Definimos $\mathcal{M}_{n}^{r}$ (resp., $\mathcal{M}_{n}^{r t}, \mathcal{M}_{n}^{r s t}, \mathcal{M}_{n}^{\text {elt }}$ ) como sendo a classe de todas as estruturas para $n$ agentes, tal que $\mathcal{K}_{i}$ é reflexiva (resp., reflexiva e transitiva; reflexiva simétrica e transitiva; euclideano, serial e transitiva).

A demonstração da correção de $\mathrm{T}_{n}$ (resp., $\mathrm{S} 4_{n}, \mathrm{~S} 5_{n}$ e $\mathrm{KD} 45_{n}$ ) em relação à classe $\mathcal{M}_{n}^{r}$ (resp., $\mathcal{M}_{n}^{r t}, \mathcal{M}_{n}^{r s t}$ e $\mathcal{M}_{n}^{\text {elt }}$ ) segue de modo semelhante à do Teorema 2.5, basta (em cada caso) completar a demonstração do Teorema $2.4 \mathrm{em}$ relação aos axiomas adicionados.

\section{Teorema 3.5.}

$\mathrm{T}_{n}$ (resp., $\mathrm{S}_{n}, \mathrm{~S}_{n}$ e $\mathrm{KD}_{4} 5_{n}$ ) é correto em relação a $\mathcal{M}_{n}^{r}$ (resp., $\mathcal{M}_{n}^{r t}$, $\mathcal{M}_{n}^{r s t}$ e $\left.\mathcal{M}_{n}^{e l t}\right)$.

Demonstração. [Halpern [36]]

Para completar a demonstração em 2.4 em relação ao axioma $T$ (resp., 4,5 e $D$ ) devemos mostrar que se $\mathrm{M}$ é elemento de $\mathcal{M}_{n}^{r}$ (resp., $\mathcal{M}_{n}^{r t}, \mathcal{M}_{n}^{r s t}$ e $\mathcal{M}_{n}^{\text {elt }}$ ), então $M$ satisfaz o axioma $T$ (resp., 4, 5 e $D$ ). Pela Proposição 3.3 cada um dos casos se verifica.

Para a completude de $\mathrm{T}_{n}$ (resp., $\mathrm{S} 4_{n}, \mathrm{~S} 5_{n}$ e $\left.\mathrm{KD} 45_{n}\right)^{2}$ em relação à classe $\mathcal{M}_{n}^{r}$ (resp., $\mathcal{M}_{n}^{r t}, \mathcal{M}_{n}^{r s t}$ e $\mathcal{M}_{n}^{\text {elt }}$ ) seguimos o esquema do Teorema 2.6.

\section{Teorema 3.6.}

$\mathrm{T}_{n}$ (resp., $\mathrm{S}_{n}, \mathrm{~S}_{n}$ e $\mathrm{KD}_{4} 5_{n}$ ) é completo em relação a $\mathcal{M}_{n}^{r}$ (resp., $\mathcal{M}_{n}^{r t}$, $\left.\mathcal{M}_{n}^{r s t}, \mathcal{M}_{n}^{\text {elt }}\right)$.

Demonstração. [Halpern [36]]

Para demonstrar que: toda fórmula consistente em $\mathrm{T}_{n}$ (resp., $\mathrm{S} 4_{n}, \mathrm{~S} 5_{n}$ e KD45n ) é satisfatível em alguma estrutura de $\mathcal{M}_{n}^{r}$ (resp., $\mathcal{M}_{n}^{r t}, \mathcal{M}_{n}^{r s t} \mathrm{e}$ $\mathcal{M}_{n}^{e l t}$ ) consideramos a estrutura $\mathrm{M}_{\text {can }}$. Podemos tomar a estrutura $\mathrm{M}_{\text {can }}$,

\footnotetext{
${ }^{2}$ A prova da completude de $S 5_{n}$ de Halpern e Moses [36] é uma extensão da prova da completude de S5 devida a Kripke (1963) e Hintikka (1962).
} 
pois pela Proposição 3.4 se $\mathrm{M}_{\text {can }}$ satisfaz os axiomas de $\mathrm{T}_{n}$ (resp., $\mathrm{S} 4_{n}, \mathrm{~S} 5_{n}$ e KD45n), então $\mathrm{M}_{\text {can }}$ é elemento de $\mathcal{M}_{n}^{r}$ (resp., $\mathcal{M}_{n}^{r t}, \mathcal{M}_{n}^{r s t}$ e $\mathcal{M}_{n}^{\text {elt }}$ ). Como na demonstração em 2.6, basta mostrar que $\left(\mathrm{M}_{\mathrm{can}}, w_{\Sigma}\right) \models \varphi$ se, e somente se, $\varphi \in \Sigma$, com $\Sigma$ conjunto maximal consistente de $\mathrm{T}_{n}$ (resp., $\mathrm{S} 4_{n}, \mathrm{~S} 5_{n}$ e $\mathrm{KD} 45_{n}$ ) . O esquema da demonstração, em todos os casos, é idêntico ao do Teorema 2.6 .

$\mathrm{Na}$ seção seguinte estudamos algumas propriedades das estruturas em $\mathcal{M}_{n}^{r}$,

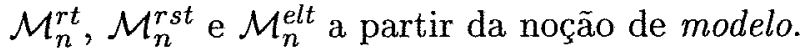

\subsection{Modelos, Fechos e Frames}

Os teoremas de correção (3.5) e completude (3.6) mostram que toda estrutura em $\mathcal{M}_{n}^{r}$ (resp., $\mathcal{M}_{n}^{r t}, \mathcal{M}_{n}^{r s t}$ e $\mathcal{M}_{n}^{\text {elt }}$ ) é modelo de $\mathrm{T}_{n}$ (resp., $\mathrm{S} 4_{n}$, $\mathrm{S} 5_{n}$ e $\mathrm{KD} 45_{n}$ ), porém a recíproca não se verifica.

\section{Definição 3.3.}

Dizemos que uma estrutura de Kripke $\mathrm{M}$ é um modelo de $\mathrm{K}_{n}$ (resp., $\mathrm{T}_{n}$, $\mathrm{S} 4_{n}, \mathrm{~S}_{n}$ e $\mathrm{KD}_{4} 5_{n}$ ) se toda fórmula dedutivel em $\mathrm{K}_{n}$ (resp., $\mathrm{T}_{n}, \mathrm{~S}_{n}, \mathrm{~S}_{n}$ e $\mathrm{KD}_{4}$ ) é válida em $\mathrm{M}$.

A seguir exemplos que mostram modelos de $\mathrm{S} 5_{n}$ e $\mathrm{KD} 45_{n}$ que não estão em $\mathcal{M}_{n}^{\text {rst }}$ e $\mathcal{M}_{n}^{\text {elt }}$, respectivamente.

\section{Exemplo 3.1.}

Considere $\Phi=\{p\}, \Delta=\{1\}, W=\{s, t\}$ e $M=\left\langle W, \pi_{W}, \mathcal{K}_{1}\right\rangle$, com $\mathcal{K}_{1}=\{(s, t),(t, t)\}$ e $\pi_{s}(p)=\pi_{t}(p)=\mathrm{T}$.

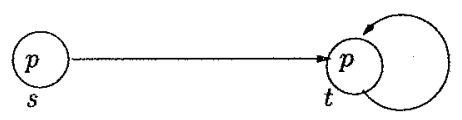

Figura 3.2: Modelo de $\mathrm{S} 5 \operatorname{com} \mathcal{K}_{1}$ não reflexivo nem simétrico.

Pelo diagrama, é fácil ver que $\mathcal{K}_{1}$ não é reflexiva, nem simétrica, porém $\mathrm{M}$ é modelo de $\mathrm{S} 5$. Veja que $(\mathrm{M}, s) \vDash \varphi$ se, e somente se, $(\mathrm{M}, t) \vDash \varphi$, para verificar basta considerar as fórmulas $\varphi$ de acordo com o número de conectivos que nela ocorrem. Se $\varphi \in \Phi$ é imediato que $(\mathrm{M}, s) \vDash \varphi$ se, e só se, $(\mathrm{M}, t) \models \varphi$, pela definição de $\pi_{s}$ e $\pi_{t}$. Se $\varphi$ da forma $\neg \psi$ ou $\psi \rightarrow \chi$ o resultado segue pela hipótese de indução. Agora, suponha que $\varphi$ é da forma $K_{i} \psi$, com $\psi$ tal que $(\mathrm{M}, s) \models \psi$ se, e só se, $(\mathrm{M}, t) \vDash \psi,(\mathrm{M}, s) \models \varphi$ e $(\mathrm{M}, t) \not \varphi$. Logo, para todo $w$ em $M$ tal que $(s, w) \in \mathcal{K}_{1},(\mathrm{M}, w) \models \psi$ e existe $w^{\prime}$ em 
$\mathrm{M}$ tal que $\left(t, w^{\prime}\right) \in \mathcal{K}_{1},\left(\mathrm{M}, w^{\prime}\right) \not \models \psi$, o que contradiz a hipótese de indução. Então, $(M, t) \models \varphi$. A recíproca é análoga. Assim, $(\mathrm{M}, s) \models \varphi$ se, e só se, $(\mathrm{M}, t) \models \varphi$.

A partir do fato anterior tem-se que $T$ e 5 são fórmulas válidas em $M$. De fato, suponha que $\mathrm{M} \not \models K_{i} \varphi \rightarrow \varphi$, então existe $w$ em $\mathrm{M}$ tal que $(\mathrm{M}, w) \vDash K_{i} \varphi$ e $(\mathrm{M}, w) \not \models \varphi$, isto é, $\left(\mathrm{M}, w^{\prime}\right) \vDash \varphi$, para todo $w^{\prime}$ em M tal que $\left(w, w^{\prime}\right) \in \mathcal{K}_{1}$. O que contradiz $(\mathrm{M}, s) \vDash \varphi$ se e só se $(\mathrm{M}, t) \vDash \varphi$. Logo, $\mathrm{M} \models K_{i} \varphi \rightarrow \varphi$. Analogamente, $\mathrm{M} \models \neg K_{i} \varphi \rightarrow K_{i} \neg K_{i} \varphi$. Portanto, $\mathrm{M}$ é modelo de S5.

Para KD45 consideramos uma variação do exemplo anterior com $\mathcal{K}_{1}$ não serial e cuja demonstração é semelhante.

\section{Exemplo 3.2.}

Considere $\Phi=\{p\}, \Delta=\{1\}, W=\{s, t, u\}$ e $\mathrm{M}=\left\langle W, \pi_{W}, \mathcal{K}_{1}\right\rangle$, com $\mathcal{K}_{1}=\{(s, t),(s, u),(t, t),(u, u)\}$ e $\pi_{s}(p)=\pi_{t}(p)=\pi_{u}(p)=\mathrm{T}$.

Ainda, os modelos $\mathrm{M}$ dos sistemas S5 e KD45, compostos por um único agente, podem ser caracterizados de uma forma muito particular. Para S5 necessitamos definir a relação universal e para KD45 uma relação que destaque um mundo $w_{0}$.

\section{Definição 3.4.}

Uma relação $\mathcal{K}_{i}$ de $\mathrm{M}$ é universal (ou completa) se para quaisquer we s em $\mathrm{M},(w, s) \in \mathcal{K}_{i}$.

Se $\mathcal{K}_{i}$ de $\mathrm{M}$ é tal que, para todo $\left(w, w^{\prime}\right) \in \mathcal{K}_{i}$, com $w \in\left\{w_{0}\right\} \cup W$, então dizemos que $\mathcal{K}_{i}$ é uma relação que destaca o mundo $w_{0}$ em $\mathrm{M}$. Chamamos $w_{0}$ de mundo real.

Assim, se $M$ é composto por uma relação $\mathcal{K}_{1}$ universal, isto é, o agente em $\mathrm{M}$ possui um único conjunto de mundos possíves a partir de qualquer mundo em $M$, então $M$ é um modelo de S5. Analogamente, se $M$ é tal que possui um mundo real que o agente o enxerga de diferentes modos a partir daquilo que ele pensa ser possível, então M é um modelo de KD45. Formalizemos estes conceitos.

\section{Proposição 3.7.}

i) Se $\varphi$ é KD45-consistente, $\varphi$ é satisfativel em $\mathrm{M}=\left\langle W \cup\left\{w_{0}\right\}, \pi_{W}, \mathcal{K}_{1}\right\rangle$, com $W$ não vazio e $\mathcal{K}_{1}=\left\{(w, s) \mid w \in W \cup\left\{w_{0}\right\}\right.$ e $\left.s \in W\right\}$.

ii) Se $\varphi$ é s5-consistente, então $\varphi$ é satisfatível em $\mathrm{M}=\left\langle W, \pi_{W}, \mathcal{K}_{1}\right\rangle$, com $\mathcal{K}_{1}$ universal. 
Extensões do Sistema $K_{n}$

Demonstração. [Halpern [36]]

i) Seja $\varphi$ KD45-consistente. Então, pelo Teorema 3.5, existe uma estrutura $\mathrm{M}^{\prime}=\left\langle W^{\prime}, \pi_{W}^{\prime}, \mathcal{K}_{1}^{\prime}\right\rangle$ em $\mathcal{M}^{\text {elt }}$ com $\mathrm{M}^{\prime} \models \varphi$. Existe $w_{0}$ em $\mathrm{M}^{\prime}\left(W^{\prime} \neq \emptyset\right)$ tal que $\left(\mathrm{M}^{\prime}, w_{0}\right) \models \varphi$. Seja $\mathcal{K}_{1}^{\prime}\left(w_{0}\right)=\left\{w \in W^{\prime} \mid\left(w_{0}, w\right) \in \mathcal{K}_{1}^{\prime}\right\}$. Definimos $\mathrm{M}=\left\langle W, \pi_{W}, \mathcal{K}_{1}\right\rangle$ a partir de $\mathcal{K}_{1}^{\prime}\left(w_{0}\right)$ do seguinte modo: $W=\left\{w_{0}\right\} \cup \mathcal{K}_{1}^{\prime}\left(w_{0}\right)$ e $\mathcal{K}_{1}=\left\{(s, t) \mid s \in\left\{w_{0}\right\} \cup \mathcal{K}_{1}^{\prime}\left(w_{0}\right)\right.$ e $\left.s \in \mathcal{K}_{1}^{\prime}\left(w_{0}\right)\right\}$. A relação $\mathcal{K}_{1}$ é serial, pois $\mathcal{K}_{1}^{\prime}\left(w_{0}\right) \neq \emptyset$. Note que para $t$ em $W$ se $t=w_{0}$ nada a fazer, se $t \neq w_{0}$ e $\left(w_{0}, t\right) \in \mathcal{K}_{1}$, então existe $t^{\prime}$ em $W$ tal que $\left(t, t^{\prime}\right) \in \mathcal{K}_{1}^{\prime}$, já que $\mathcal{K}_{1}^{\prime}$ é serial e pela transitividade $\left(t, t^{\prime}\right) \in \mathcal{K}_{1}$, portanto $\mathcal{K}_{1}$ é serial. De $\mathcal{K}_{1}^{\prime}$ ser euclideana decorre que para quaisquer $s$ e $t$ em $\mathcal{K}_{1}^{\prime}\left(w_{0}\right)(s, t) \in \mathcal{K}_{1}^{\prime}$ e $(s, t) \in \mathcal{K}_{1}$, logo $\mathcal{K}_{1}$ é euclideana. A transitividade de $\mathcal{K}_{1}$, segue da transitividade de $\mathcal{K}_{1}^{\prime}$, pois se $s$ em $\mathcal{K}_{1}^{\prime}\left(w_{0}\right)$ e $(s, t) \in \mathcal{K}_{1}$, então $t$ em $\mathcal{K}_{1}^{\prime}\left(w_{0}\right)$. Portanto, $\mathcal{K}_{1}$ é euclideana, transitiva e serial, isto é, $M \in \mathcal{M}^{\text {elt }}$, ou seja, se $\varphi$ é KD45-consistente, então $\mathrm{M} \models \varphi$, em particular $\left(\mathrm{M}, w_{0}\right) \models \varphi$. Observe que $\left(\mathrm{M}^{\prime}, w_{0}\right) \models \varphi$ se, e somente se, $\left(\mathrm{M}, w_{0}\right) \models \varphi$. A demonstração é por indução no número de conectivos de $\varphi$.

- A base da indução é sobre as fórmulas $\varphi \in \Phi$. Lembrando que $\pi_{w}^{\prime}$ é restrição de $\pi_{w}$ a $W$. Logo, $(\mathrm{M}, w) \vDash \varphi$ se e somente se $\pi_{w}(\varphi)=T$ se e só se $\pi_{w}^{\prime}(\varphi)=T$ se e somente se $(\mathrm{M}, w) \vDash \varphi$, para qualquer $w$ de $M$.

- Se $\varphi$ é da forma $\neg \psi$ ou $\psi \rightarrow \chi$ decorre diretamente da base que $\left(\mathrm{M}^{\prime}, w\right) \models \varphi$ se e só se $(\mathrm{M}, w) \models \varphi$, para qualquer $w$ de $\mathrm{M}$.

- Seja $\varphi$ da forma $K_{i} \psi$ e $\psi$ tal que $\left(\mathrm{M}^{\prime}, w\right) \models \psi$ se e somente se $(\mathrm{M}, w) \models \psi$, para qualquer $w$ de $\mathrm{M}$. Suponha $\left(\mathrm{M}^{\prime}, w\right) \models K_{i} \psi$. Então, para todo $s$ de $M^{\prime}$ tal que $(w, s) \in \mathcal{K}_{1}^{\prime}$ temos que $\left(\mathrm{M}^{\prime}, s\right) \vDash \psi$. Pela hipótese de indução $(\mathrm{M}, s) \vDash \psi$ e de $\mathcal{K}_{1} \subseteq \mathcal{K}_{1}^{\prime}$ tem-se $(\mathrm{M}, w) \models K_{i} \psi$, para qualquer $w$ de $\mathrm{M}$.

Agora, suponha $(\mathrm{M}, w) \models K_{i} \psi$ e $s$ em $\mathrm{M}^{\prime}$ tal que $(w, s) \in \mathcal{K}_{1}^{\prime}$, então ou $(w, s) \in \mathcal{K}_{1}$ ou $(w, s) \notin \mathcal{K}_{1}$ :

- se $(w, s) \in \mathcal{K}_{1}$, de $(\mathrm{M}, w) \vDash K_{i} \psi$ temos $(\mathrm{M}, s) \vDash \psi$ e pela hipótese de indução $\left(\mathrm{M}^{\prime}, s\right) \models \psi$,

- e não ocorre $(w, s) \notin \mathcal{K}_{1}$, pois como $w \in \mathcal{K}_{1}^{\prime}\left(w_{0}\right)$, então $s \in$ $\mathcal{K}_{1}^{\prime}\left(w_{0}\right)$ pela transitividade de $\mathcal{K}_{1}^{\prime}$.

Então, $\left(M^{\prime}, w\right) \vDash \varphi$, para qualquer $w$ de $M$.

Portanto, $\left(\mathrm{M}^{\prime}, w\right) \vDash \varphi$ se, e só se, $(\mathrm{M}, w) \vDash \varphi$, para todo $w$ de $\mathrm{M}$.

ii) Para o caso de $\mathrm{S} 5$ a demonstração é análoga. Basta observar que de $M^{\prime} \in \mathcal{M}^{r s t}$ tem-se $\mathcal{K}_{1}^{\prime}$ reflexivo e transitivo, então, pela Proposição 
$3.2, \mathcal{K}_{1}^{\prime}$ é euclideano; logo, para quaisquer $s$ e $t$ em $\mathcal{K}_{1}^{\prime}\left(w_{0}\right)(s, t) \in \mathcal{K}_{1}^{\prime}$ e $(s, t) \in \mathcal{K}_{1}$ e $\mathcal{K}_{1}$ é universal. De modo semelhante, mostra-se que $\left(\mathrm{M}^{\prime}, w\right) \models \varphi$ se e só se $(\mathrm{M}, w) \models \varphi$, para todo $w$ de $\mathrm{M}$.

\section{Observação 3.3 .}

A proposição anterior mostra que os modelos $\mathrm{M}$ de $\mathrm{S} 5$ independem da relação $\mathcal{K}_{1}$, isto é, podemos defini-los por $\mathrm{M}=\left\langle W, \pi_{W}\right\rangle$ sem fazer referência à relação de acessibilidade, pois para quaisquer $w$ e s em $W$ o par $(w, s) \in \mathcal{K}_{1}$. De mesmo modo, os modelos $\mathrm{M}$ de KD45 tem o formato $\mathrm{M}=\left\langle W, w_{0}, \pi_{W}\right\rangle$. Essa caracterização dos modelos de S5 e KD45 influem diretamente no problema de decisão (5.1) desses sistemas.

Os modelos de sistemas com mais de um agente não possuem uma descrição como as da proposição anterior (3.7), no entanto podemos obter algumas dicas pela análise da recíproca da afirmação: toda estrutura em $\mathcal{M}_{n}^{r}$ (resp., $\mathcal{M}_{n}^{r t}, \mathcal{M}_{n}^{r s t}$ e $\mathcal{M}_{n}^{\text {elt }}$ ) é modelo de $\mathrm{T}_{n}$ (resp., $\mathrm{S}_{n}, \mathrm{~S}_{n}$ e $\mathrm{KD} 45_{n}$ ). Os dois últimos exemplos (3.1 e 3.2) mostram que a recíproca é falsa, porém podemos considerá-la verdadeira nos seguintes sentidos:

i) todo modelo $\mathrm{M}$ de $\mathrm{T}_{n}$ (resp., $\mathrm{S} 4_{n}, \mathrm{~S} 5_{n}$ e $\mathrm{KD} 45_{n}$ ) possui uma estrutura $\mathrm{M}_{\mathrm{r}}$ (resp., $\mathrm{M}_{\mathrm{rt}}, \mathrm{M}_{\mathrm{rst}}$ e $\mathrm{M}_{\mathrm{elt}}$ ) em $\mathcal{M}_{n}^{r}$ (resp., $\mathcal{M}_{n}^{r t}, \mathcal{M}_{n}^{r s t}$ e $\mathcal{M}_{n}^{\text {elt }}$ ) equivalente a $\mathrm{M}$,

ii) todo modelo $\mathrm{M}$ de $\mathrm{T}_{n}$ (resp., $\mathrm{S} 4_{n}, \mathrm{~S} 5_{n}$ e $\mathrm{KD} 45_{n}$ ) é baseado num frame $F$, e um frame $F$ é modelo de $\mathrm{T}_{n}$ (resp., $\mathrm{S} 4_{n}, \mathrm{~S} 5_{n}$ e $\mathrm{KD}_{4} 5_{n}$ ) se, e somente se, $F$ pertence à $\mathcal{F}_{n}^{r}$ (resp., $\mathcal{F}_{n}^{r t}, \mathcal{F}_{n}^{r s t}$ e $\mathcal{F}_{n}^{\text {elt }}$ ).

Formalizamos estas duas idéias nos teoremas que se seguem (3.8 e 3.9). Primeiramente, definimos as noções de fecho e estrutura equivalente, enunciamos e demonstramos o Teorema 3.8. Caracterizamos fechos como modelos, determinamos as classes $\mathcal{F}_{n}, \mathcal{F}_{n}^{r}, \mathcal{F}_{n}^{r t}, \mathcal{F}_{n}^{r s t}$ e $\mathcal{F}_{n}^{\text {elt }}$ para finalmente enunciar e demonstrar que: $F$ é modelo de $\mathrm{T}_{n}$ (resp., $\mathrm{S}_{n}, \mathrm{~S}_{n}$ e $\mathrm{KD}_{4} 5_{n}$ ) se, e somente se, $F$ pertence $\grave{a} \mathcal{F}_{n}^{r}$ (resp., $\mathcal{F}_{n}^{r t}, \mathcal{F}_{n}^{r s t}$ e $\mathcal{F}_{n}^{\text {elt }}$ ) (Teorema 3.9).

\section{Definição 3.5.}

$O$ fecho reflexivo de uma estrutura $\mathrm{M}$, com $\mathrm{M}=\left\langle W, \pi_{W}, \mathcal{K}_{1}, \ldots, \mathcal{K}_{n}\right\rangle$, é a estrutura $\mathrm{M}_{\mathrm{r}}, \operatorname{com} \mathrm{M}_{\mathrm{r}}=\left\langle W, \pi_{W}, \mathcal{K}_{1}^{r}, \ldots, \mathcal{K}_{n}^{r}\right\rangle$, e $\mathcal{K}_{i}^{r}$ o fecho reflexivo de $\mathcal{K}_{i}$, isto é, $\mathcal{K}_{i}^{r}=\mathcal{K}_{i} \cup\{(w, w) \in W \times W \mid w \in W\}$. De modo análogo, definimos o rt-fecho (resp., rst-fecho, et-fecho) de $\mathrm{M}$, como sendo a estrutura $\mathrm{M}_{\mathrm{rt}}$ (resp., $\mathrm{M}_{\mathrm{rst}}, \mathrm{M}_{\mathrm{et}}$ ) tal que suas relações são rt-fechos (resp., rst-fechos e et-fechos) das relações $\mathcal{K}_{i}$ em $\mathrm{M}$. 


\section{Teorema 3.8.}

Dizemos que $(\mathrm{M}, w)$ e $\left(\mathrm{M}^{\prime}, w^{\prime}\right)$ são equivalentes se, para toda fórmula $\varphi$ : $(\mathrm{M}, w) \models \varphi$ se, e somente se, $\left(\mathrm{M}^{\prime}, w^{\prime}\right) \models \varphi$. Notação: $(\mathrm{M}, w) \equiv\left(\mathrm{M}^{\prime}, w^{\prime}\right)$.

i) se $\mathrm{M}$ é um modelo de $\mathrm{T}_{n}$ e $\mathrm{M}_{\mathrm{r}}$ o seu r-fecho, com $\mathrm{M}_{\mathrm{r}} \in \mathcal{M}_{n}^{r}$, então $(\mathrm{M}, w) \equiv\left(\mathrm{M}_{\mathrm{r}}, w\right)$, para qualquer $w$ de $\mathrm{M}$,

ii) se $\mathrm{M}$ é um modelo de $\mathrm{S}_{n}$ e $\mathrm{M}_{\mathrm{rt}}$ o seu rt-fecho, com $\mathrm{M}_{\mathrm{rt}} \in \mathcal{M}_{n}^{r t}$, então $(\mathrm{M}, w) \equiv\left(\mathrm{M}_{\mathrm{rt}}, w\right)$, para todo $w$ de $\mathrm{M}$,

iii) se $\mathrm{M}$ é um modelo de $\mathrm{S}_{n}$ e $\mathrm{M}_{\mathrm{rst}}$ o seu rst-fecho, com $\mathrm{M}_{\mathrm{rst}} \in \mathcal{M}_{n}^{\text {rst }}$, então $(\mathrm{M}, w) \equiv\left(\mathrm{M}_{\mathrm{rst}}, w\right)$, para todo $w$ de $\mathrm{M}$,

iv) se $\mathrm{M}$ é um modelo de $\mathrm{KD}_{4}$ e $\mathrm{M}_{\mathrm{et}}$ o seu et-fecho, com $\mathrm{M}_{\mathrm{et}} \in \mathcal{M}_{n}^{\text {elt }}$, então $(\mathrm{M}, w) \equiv\left(\mathrm{M}_{\mathrm{et}}, w\right)$, para todo $w$ de $\mathrm{M}$.

\section{Demonstração.}

i) Seja $M$ um modelo de $T_{n}$ e $M_{r}$ o seu r-fecho, com $M_{r} \in \mathcal{M}_{n}^{r}$. Devemos mostrar, que para toda fórmula $\varphi$ de $\mathrm{T}_{n}$, tem-se que $(\mathrm{M}, w) \vDash \varphi$ se, e somente se, $\left(\mathrm{M}_{\mathrm{r}}, w\right) \vDash \varphi$, para qualquer $w$ de M. A demonstração é por indução no número de conectivos de $\varphi$.

A base da indução é sobre as fórmulas $\varphi \in \Phi$. $(\mathrm{M}, w) \models \varphi$ se, e somente se, $\pi_{w}(\varphi)=T$ se, e só se, $\left(M_{r}, w\right) \models \varphi$, para qualquer $w$ de $M$.

Se $\varphi$ é da forma $\neg \psi$ ou $\psi \rightarrow \chi$, decorre diretamente da base que $(\mathrm{M}, w) \models \varphi$ se, e só se, $\left(\mathrm{M}_{\mathrm{r}}, w\right) \models \varphi$, para qualquer $w$ de $\mathrm{M}$.

Seja $\varphi$ da forma $K_{i} \psi$, com $\psi$ tal que $(\mathrm{M}, w) \vDash \psi$ se, e só se, $\left(\mathrm{M}_{\mathrm{r}}, w\right) \vDash \psi$, para qualquer $w$ de $\mathrm{M}$. Suponha que $\left(\mathrm{M}_{\mathrm{r}}, w\right) \models K_{i} \psi$. Então, para todo $s$ de $M_{r}$ tal que $(w, s) \in \mathcal{K}_{i}$ temos que $\left(M_{r}, w\right) \models \psi$. Como $\mathcal{K}_{i} \subseteq \mathcal{K}_{i}^{r}$, então $(\mathrm{M}, w) \models K_{i} \psi$.

Agora, $\operatorname{sejam}(\mathrm{M}, w) \vDash K_{i} \psi$ e $s$ em $\mathrm{M}_{\mathrm{r}}$, tal que $(w, s) \in \mathcal{K}_{i}^{r}$; então ou $(w, s) \in \mathcal{K}_{i}$ ou $w=s$ :

- se $(w, s) \in \mathcal{K}_{i}$, como $(\mathrm{M}, w) \models K_{i} \psi$, temos $(\mathrm{M}, s) \models \psi$ e, pela hipótese de indução, $\left(\mathrm{M}_{\mathrm{r}}, s\right) \models \psi$,

- de $\mathrm{M}$ ser um modelo de $\mathrm{T}_{n}$ segue $(\mathrm{M}, s) \models K_{i} \psi \rightarrow \psi$. Da hipótese e de $w=s \operatorname{temos}(\mathrm{M}, s) \vDash K_{i} \psi$, ou seja, $(\mathrm{M}, s) \models \psi$, logo, pela hipótese de indução, $\left(\mathrm{M}_{\mathrm{r}}, s\right) \models \psi$.

Portanto, $(\mathrm{M}, w) \vDash \varphi$ se, e somente se, $\left(\mathrm{M}_{\mathrm{r}}, w\right) \vDash \varphi$, para qualquer $w$ de $\mathrm{M}$, isto é, $(\mathrm{M}, w) \equiv\left(\mathrm{M}_{\mathrm{r}}, w\right)$, para qualquer $w$ de $\mathrm{M}$.

ii) Seja $\mathrm{M}$ um modelo de $S 4_{n}$ e $\mathrm{M}_{\mathrm{rt}}$ o seu rt-fecho, com $\mathrm{M}_{\mathrm{rt}} \in \mathcal{M}_{n}^{r t}$. A demonstração segue o esquema do item anterior, é idêntica para os 
casos $\varphi \in \Phi, \varphi$ da forma $\neg \psi$ ou $\psi \rightarrow \chi$ e $\varphi$ da forma $K_{i} \psi$ supondo $\left(\mathrm{M}_{\mathrm{rt}}, w\right) \vDash K_{i} \psi$.

Para o caso $\varphi$ da forma $K_{i} \psi, \operatorname{com}(\mathrm{M}, w) \models K_{i} \psi$ e $s$ em $\mathrm{M}_{\mathrm{rt}}$, tal que $(w, s) \in \mathcal{K}_{i}^{r t}$, temos que avaliar as seguinte possibilidades:

- ou $(w, s) \in \mathcal{K}_{i}$ (análogo ao item anterior),

- ou $w=s$. Como $\mathrm{M}$ é modelo de $S 4_{n}$, então M satisfaz $T$ e segue que $\left(\mathrm{M}_{\mathrm{rt}}, s\right) \vDash \psi$,

- ou existe $w^{\prime}$ em $\mathrm{M}$, tal que $\left(w, w^{\prime}\right),\left(w^{\prime}, s\right) \in \mathcal{K}_{i}^{r t}$. Como $(\mathrm{M}, w) \models K_{i} \psi \rightarrow K_{i} K_{i} \psi$ e $(\mathrm{M}, w) \models K_{i} \psi$ (hipótese), temos $(\mathrm{M}, w) \models K_{i} K_{i} \psi$. Isto é, $\left(\mathrm{M}, w^{\prime}\right) \models K_{i} \psi,(\mathrm{M}, s) \models \psi$ e segue da hipótese de indução que $\left(\mathrm{M}_{\mathrm{rt}}, s\right) \vDash \psi$.

Portanto, $(\mathrm{M}, w) \equiv\left(\mathrm{M}_{\mathrm{rt}}, w\right)$ para todo $w$ de $\mathrm{M}$,

iii) A demonstração é como nos itens anteriores, restando verificar as possibilidades relacionadas à simetria e transitividade.

- Basta analisar a possiblidade relativa à $\mathcal{K}_{i}^{r s t}$ ser euclideana (Prop. $3.2)$, isto é, que existe $w^{\prime}$ tal que $\left(w^{\prime}, w\right),\left(w^{\prime}, s\right) \in \mathcal{K}_{i}^{r s t}$. Como $\mathrm{M}$ é modelo de $\mathrm{S} 5_{n}$ segue que $\left(\mathrm{M}, w^{\prime}\right) \models \neg K_{i} \psi \rightarrow K_{i} \neg K_{i} \psi \mathrm{e}$ $(\mathrm{M}, w) \models K_{i} \psi$ ou $(\mathrm{M}, w) \models K_{i} \neg K_{i} \psi$. Porém, $\left(w^{\prime}, w\right) \in \mathcal{K}_{i}^{r s t} \mathrm{e}$ $(\mathrm{M}, w) \vDash K_{i} \psi$, então $\left(\mathrm{M}, w^{\prime}\right) \vDash K_{i} \psi$, isto é, $(\mathrm{M}, s) \vDash \psi$.

Portanto, $(\mathrm{M}, w) \equiv\left(\mathrm{M}_{\mathrm{rst}}, w\right)$ para todo $w$ de $\mathrm{M}$,

iv) Seja $M$ modelo de $K D 45_{n}$ e $M_{\text {et }}$ o seu et-fecho, com $M_{\text {et }} \in \mathcal{M}_{n}^{\text {elt }}$. De $\mathrm{M}$ ser modelo de $\mathrm{KD} 45_{n}$, dos itens (ii) e (iii), segue que $(\mathrm{M}, w) \equiv\left(\mathrm{M}_{\mathrm{et}}, w\right)$, para todo $w$ de $\mathrm{M}$. Como $\mathrm{M}$ satisfaz $D$, então $\mathcal{K}_{i}$ é serial (Prop. 3.4).

Para podermos escrever os resultados enunciados sobre estruturas em termos de moldes (frames), necessitamos definir as classes de frames, lembrando que um molde é como uma estrutura de Kripke sem a referência $\pi_{W}$ e que sua utilização para caracterizar sistemas axiomáticos é usual (vide van Benthem [83] e Blackburn [2]).

\section{Definição 3.6.}

Dizemos que um frame (ou molde) F é um modelo de $\mathrm{K}_{n}$ (resp., $\mathrm{T}_{n}, \mathrm{~S} 4_{n}$, $\mathrm{S}_{n}$ e $\mathrm{KD}_{4} 5_{n}$ ) se toda estrutura $\mathrm{M}$ baseada em $F$ é modelo de $\mathrm{K}_{n}$ (resp., $\mathrm{T}_{n}, \mathrm{~S}_{n}, \mathrm{~S}_{n}$ e $\left.\mathrm{KD}_{4} 5_{n}\right)$. Denotamos por $\mathcal{F}_{n}$ a classe de todos os frames, similarmente à definição das classes de estruturas denotamos as subclasses de $\mathcal{F}_{n}$ por $\mathcal{F}_{n}^{r}, \mathcal{F}_{n}^{r t}, \mathcal{F}_{n}^{r s t}$ e $\mathcal{F}_{n}^{\text {elt }}$. 


\section{Teorema 3.9.}

$F$ é um modelo de $\mathrm{T}_{\mathrm{n}}$ (resp., $\mathrm{S}_{\mathrm{n}}, \mathrm{S}_{\mathrm{n}}$ e $\mathrm{KD} 45_{n}$ ) se, e só se, $F$ é elemento de $\mathcal{F}_{n}^{r}$ (resp., $\mathcal{F}_{n}^{r t}, \mathcal{F}_{n}^{r s t}$ e $\left.\mathcal{F}_{n}^{\text {elt }}\right)$.

\section{Demonstração.}

Segue dos Teoremas 3.5 e 3.6 que se $F \in \mathcal{F}_{n}^{r}$, então $F$ é um modelo de $T_{n}$. Para a recíproca, supomos $F=\left\langle W, \mathcal{K}_{1}, \ldots, \mathcal{K}_{n}\right\rangle$ modelo de $\mathrm{T}_{n}$ e construímos uma estrutura $\mathrm{M}$ a partir da suposição de que $F \notin \mathcal{F}_{n}^{r}$, a fim de obtermos uma contradição. Basicamente, $M$ não deve satisfazer o axioma $T$. Se $F \notin \mathcal{F}_{n}^{r}$, então existe $w \in W$ tal que, para algum $i,(w, w) \notin \mathcal{K}_{i}$. Tomemos uma proposição primitiva $p$ e escolhemos $\pi_{w}{ }^{3}$ tal que para qualquer $s \in W(w \neq s) \pi_{w}(p)=\perp$ e $\pi_{t}(p)=T$. Seja $q \in \Phi, q \neq p$ e verdadeira sob qualquer interpretação em $\pi_{W}$. A estrutura $\mathrm{M}=\left\langle W, \pi_{W}, \mathcal{K}_{1}, \ldots, \mathcal{K}_{n}\right\rangle$ é baseada em $F$ e $(\mathrm{M}, w) \not \forall K_{i} p \rightarrow p$, contradizendo o fato de $F$ ser um modelo de $\mathrm{T}_{n}$. Portanto, $F$ é um modelo de $\mathrm{T}_{n}$ se, e só se, $F \in \mathcal{F}_{n}^{r}$. A demonstração dos outros itens é análoga.

$\mathrm{O}$ último teorema sugere que o estudo dos sistemas $\mathrm{K}_{n}, \mathrm{~T}_{n}, \mathrm{~S} 4_{n}$, $\mathrm{S} 5_{n}$ e $\mathrm{KD}_{4} 5_{n}$ sob a óptica dos frames, em certos casos, é mais apropriada que a óptica das estruturas (porém, com os devidos cuidados em relação às regras de inferência, como mencionamos na Observação 2.4). Por exemplo, o problema da validade (vide Nota 3 do capítulo 5) torna-se interessante sob a visão de frames.

\subsection{Estruturas Finitas}

As estruturas $M_{\text {can }}$ que utilizamos para demonstrar a correção e completude dos sistemas de conhecimento não são efetivamente construtíveis, pois são infinitas. Nesta seção tratamos de estruturas finitas que satisfaçam a propriedades de correção e completude.

\section{Definição 3.7.}

Seja $\mathrm{M}=\left\langle W, \pi_{W}, \mathcal{K}_{1}, \ldots, \mathcal{K}_{n}\right\rangle$ e $\Delta=\{1,2, \ldots, n\}$. Definimos o tamanho, $(|\mathrm{M}| \mid)$, de $\mathrm{M}$ como sendo a soma $|W|+\sum_{i \in \Delta}\left|\mathcal{K}_{i}\right|$.

\footnotetext{
${ }^{3} \mathrm{~A}$ escolha pode ser feita conforme comentários feitos no início da seção 2.2 , segundo parágrafo.
} 
Necessitamos definir certos conjuntos para nossos objetivos.

\section{Definição 3.8.}

Dada uma fórmula $\varphi$ denotamos:

- $\operatorname{Sub}(\varphi)$ como sendo o conjunto de todas as subfórmulas de $\varphi$,

- $S u b\urcorner(\varphi)=S u b(\varphi) \cup\{\neg \psi: \psi \in S u b(\varphi)\}$,

- Con $(\varphi)$ o conjunto formado por todos os subconjuntos consistentes e maximais em relação a $S u b\urcorner(\varphi)$,

Os conjuntos acima descritos são finitos como mostra a proposição a seguir.

\section{Proposição 3.10.}

Dada uma fórmula $\varphi$ temos que:

i) $|\operatorname{Sub}(\varphi)| \leq|\varphi|$,

ii) todo subconjunto consistente de $S u b\urcorner(\varphi)$ pode ser estendido a um elemento de Con $(\varphi)$,

iii) se $\Sigma \in \operatorname{Con}(\varphi)$, então para qualquer $\psi$ de $S u b\urcorner(\varphi)$, temos que $\Sigma$ contém $\psi$ ou $\neg \psi$, mas não ambos,

iv) $|\operatorname{Con}(\varphi)| \leq 2^{|\varphi|}$.

\section{Demonstração.}

Para ver que $|S u b(\varphi)| \leq|\varphi|$ basta uma indução sobre o tamanho de $\varphi$. Para mostrar que todo subconjunto consistente de $S u b\urcorner(\varphi)$ pode ser estendido a um elemento de $\operatorname{Con}(\varphi)$ tome $\Gamma \subseteq S u b\urcorner(\varphi)$; então, pelo lema de Lindenbaum, $\Gamma$ pode ser estendido a um conjunto $\Sigma$ maximal consistente e, tomando $\Sigma^{\prime}$ como sendo a intersecção $\left.\Sigma \cap S u b\right\urcorner(\varphi)$, temos que $\Sigma^{\prime}$ é consistente e maximal em $S u b^{\urcorner}(\varphi)$, $\operatorname{logo} \Sigma^{\prime} \in \operatorname{Con}(\varphi)$ e segue que, para qualquer $\psi$ de $S u b\urcorner(\varphi), \Sigma^{\prime}$ contém $\psi$ ou $\neg \psi$, mas não ambos (Prop. 2.3). É imediato dos dois útimos itens que $|\operatorname{Con}(\varphi)| \leq 2^{|\operatorname{Sub}(\varphi)|}$, $\log |\operatorname{Con}(\varphi)| \leq 2^{|\varphi|}$.

A partir dos conjuntos acima podemos contruir estruturas finitas como mostra a proposição seguinte.

\section{Proposição 3.11 (Estruturas Finitas).}

Dada uma fórmula $\varphi$, a estrutura $\mathrm{M}_{\varphi}=\left\langle W, \pi_{W}, \mathcal{K}_{1}, \ldots, \mathcal{K}_{n}\right\rangle$, definida a seguir, possui no máximo $2^{|\varphi|}$ mundos distintos e toda letra proposicional em $\Phi$ que não ocorre em $S u b(\varphi)$ é falsa em qualquer mundo dessa estrutura. 


$$
\begin{gathered}
W=\left\{w_{\Gamma} \mid \Gamma \in \operatorname{Con}(\varphi)\right\} \\
\pi_{w_{\Gamma}}(\varphi)= \begin{cases}T & \text { se } \varphi \in \Gamma \\
\perp & \text { se } \varphi \notin \Gamma\end{cases} \\
\mathcal{K}_{i}=\left\{\left(w_{\Gamma}, w_{\Gamma^{\prime}}\right) \mid \Gamma / K_{i} \subseteq \Gamma^{\prime}\right\}, \operatorname{com} \Gamma / K_{i}=\left\{\varphi \mid K_{i} \varphi \in \Gamma\right\} .
\end{gathered}
$$

\section{Demonstração.}

Cada mundo em $\mathrm{M}_{\varphi}$ é determinado por um elemento de Con $(\varphi)$, como $|C o n(\varphi)| \leq 2^{|\varphi|}$ (Prop. 3.10), segue que há no máximo $2^{|\varphi|}$ mundos distintos em $\mathrm{M}_{\varphi}$. Toda letra proposicional em $\Phi$ que não ocorre em $S u b(\varphi)$ é falsa em qualquer mundo $w_{\Gamma}$ de $\mathrm{M}_{\varphi}$, pela definição de $\pi_{w_{\Gamma}}$.

Pela proposição, dado $|\Delta|=n$ e uma fórmula $\varphi$, a estrutura $\mathrm{M}_{\varphi}$ é finita, pois seu tamanho $(\|\mathrm{M}\|)$ é no máximo $2^{|\varphi|}+n \cdot 2^{2 \cdot|\varphi|}$.

O exemplo seguinte mostra que as relações $\mathcal{K}_{i}$ como definidas em $\mathrm{M}_{\varphi}$ podem não satisfazer as propriedades exigidas em cada um dos sistemas.

\section{Exemplo 3.3.}

Considere $\Phi=\{p\}, \Delta=\{1\}, \varphi$ a fórmula $K_{1} p$ em $\mathrm{S}_{n}(\Phi)$ e $\mathrm{M}_{\varphi}$, entẫo $\mathcal{K}_{1}$ não é transitivo. Os elementos de Con $(\varphi)$ são $\Sigma=\left\{K_{1} p, p\right\}$ $\Sigma^{\prime}=\left\{\neg K_{1} p, p\right\}$ e $\Sigma^{\prime \prime}=\left\{\neg K_{1} p, \neg p\right\}$, então não existe extensão maximal $54_{n}$-consistente, considerando as fórmulas em $\Sigma$ e $\Sigma^{\prime}$. De fato, qualquer extensão $\mathrm{S4}_{n}$-consistente de $\Sigma$ deve conter $K_{1} K_{1}$ p, o que não condiz com as fórmulas em $\Sigma^{\prime}$. Em particular, note que $\Sigma / K_{1} \subseteq \Sigma^{\prime}$ e $\Sigma^{\prime} / K_{1} \subseteq \Sigma^{\prime \prime}$, mas $\Sigma / K_{1} \nsubseteq \Sigma^{\prime \prime}$.

Logo, as estruturas $M_{\varphi}$, como definidas anteriormente, não se comportam como $\mathrm{M}_{\text {can }}$ e temos que adequar as relações $\mathcal{K}_{i}$ para cada sistema.

\section{Proposição 3.12.}

Considere $\mathrm{M}_{\varphi}$, como definida anteriormente, exceto pelas relações $\mathcal{K}_{i}$.

i) Se $\mathcal{K}_{i}=\left\{\left(w_{\Gamma}, w_{\Gamma^{\prime}}\right) \mid \Gamma / K_{i} \subseteq \Gamma^{\prime}\right\}$ e $\mathrm{M}_{\varphi} \vDash K_{i} \psi \rightarrow \psi$, então $\mathcal{K}_{i}$ é reflexivo.

ii) $\operatorname{Seja} \mathcal{K}_{i}=\left\{\left(w_{\Gamma}, w_{\Gamma^{\prime}}\right) \mid \Gamma / K_{i} \subseteq \Gamma^{\prime} / K_{i}\right\}$. Se $\mathrm{M}_{\varphi} \models K_{i} \psi \rightarrow \psi$, então $\Gamma / K_{i} \subseteq \Gamma^{\prime}$

iii) Se $\mathcal{K}_{i}=\left\{\left(w_{\Gamma}, w_{\Gamma^{\prime}}\right) \mid \Gamma / K_{i} \subseteq \Gamma^{\prime} / K_{i}\right\}$, então $\mathcal{K}_{i}$ é transitivo.

iv) Se $\mathcal{K}_{i}=\left\{\left(w_{\Gamma}, w_{\Gamma^{\prime}}\right) \mid \Gamma / K_{i}=\Gamma^{\prime} / K_{i}\right\}$, então $\mathcal{K}_{i}$ é relação de equivalência. 
v) Seja $\mathcal{K}_{i}=\left\{\left(w_{\Gamma}, w_{\Gamma^{\prime}}\right) \mid \Gamma / K_{i}=\Gamma^{\prime} / K_{i}\right.$ e $\left.\Gamma / K_{i} \subseteq \Gamma^{\prime}\right\}$, então $\mathcal{K}_{i}$ é transitivo e euclideano. Ainda, se tivermos que $\mathrm{M}_{\varphi} \models K_{i} \psi \rightarrow \neg K_{i} \neg \psi$, $\mathrm{M}_{\varphi} \models K_{i} \psi \rightarrow K_{i} K_{i} \psi$ e $\mathrm{M}_{\varphi} \models \neg K_{i} \psi \rightarrow K_{i} \neg K_{i} \psi$, então $\mathcal{K}_{i}$ é serial.

\section{Demonstração.}

i) A hipótese é que se $\mathrm{M}_{\varphi} \vDash K_{i} \psi$, então $\mathrm{M}_{\varphi} \vDash \psi$, isto é, para todo $\Gamma \in \operatorname{Con}(\varphi)$ se $\left(\mathrm{M}_{\varphi}, w_{\Gamma}\right) \vDash K_{i} \psi$, então $\left(\mathrm{M}_{\varphi}, w_{\Gamma}\right) \vDash \psi$. Então, se supormos que $\psi \in \Gamma / K_{i}$, ou seja, $K_{i} \psi \in \Gamma$, pela hipótese temos que $\psi \in \Gamma$ (note que $\psi \in S u b(\varphi)$ ). Portanto, $\Gamma / K_{i} \subseteq \Gamma$ e $\mathcal{K}_{i}$ é reflexivo.

ii) Supondo $\psi \in \Gamma / K_{i}$ temos que $\psi \in \Gamma^{\prime} / K_{i}$ e, como no item anterior, o fato de $\mathrm{M}_{\varphi} \vDash K_{i} \psi \rightarrow \psi$ nos permite afirmar que $\psi \in \Gamma^{\prime}$. Portanto, $\Gamma / K_{i} \subseteq \Gamma^{\prime}$.

iii) A transitividade de $\mathcal{K}_{i}$, como definida, é imediata.

iv) É imediato, da definição, que $\mathcal{K}_{i}$ é relação de equivalência.

v) A transitividade e a propriedade euclideana de $\mathcal{K}_{i}$ decorrem diretamente da definição. Para verificarmos se $\mathcal{K}_{i}$ é serial tomemos $\Gamma$ em $\operatorname{Con}(\varphi)$ e $\psi \in \Gamma / K_{i}$, então $K_{i} \psi \in \Gamma$. Da hipótese $\mathrm{M}_{\varphi} \vDash K_{i} \psi \rightarrow \neg K_{i} \neg \psi$ tem-se que $\left(\mathrm{M}_{\varphi}, w_{\Gamma}\right) \models \neg K_{i} \neg \psi$. Ou seja, existe $w_{\Gamma^{\prime}}$ em $\mathrm{M}_{\varphi}$ tal que $\left(w_{\Gamma}, w_{\Gamma^{\prime}}\right) \in \mathcal{K}_{i}$ e $\left(\mathrm{M}_{\varphi}, w_{\Gamma^{\prime}}\right) \models \psi$. Portanto, existe $\Gamma^{\prime}$ em $\operatorname{Con}(\varphi)$ tal que $\Gamma / K_{i} \subseteq \Gamma^{\prime}$. Vale a igualdade, $\Gamma / K_{i}=\Gamma^{\prime} K_{i}$, pois se $K_{i} \psi \in \Gamma$, temos da hipótese $\mathrm{M}_{\varphi} \models K_{i} \psi \rightarrow K_{i} K_{i} \psi$ que $\left(\mathrm{M}_{\varphi}, w_{\Gamma}\right) \vDash K_{i} K_{i} \psi, \operatorname{logo} K_{i} K_{i} \psi \in \Gamma, K_{i} \psi \in \Gamma / K_{i}$ e $K_{i} \psi \in \Gamma^{\prime}$. Então, $\psi \in \Gamma^{\prime} / K_{i}$. O outro lado: suponha $K_{i} \psi \in \Gamma^{\prime}$ e $\psi \notin \Gamma / K_{i}$, logo $K_{i} \psi \notin \Gamma$ e pela hipótese $\mathrm{M}_{\varphi} \models \neg K_{i} \psi \rightarrow K_{i} \neg K_{i} \psi$ decorre que $\left(\mathrm{M}_{\varphi}, w_{\Gamma}\right) \vDash K_{i} \neg K_{i} \psi$. Ou seja, $\neg K_{i} \psi \in \Gamma / K_{i}$ e $\neg K_{i} \psi \in \Gamma^{\prime}$, o que contradiz o fato de $\Gamma^{\prime}$ ser consistente. Portanto, segue a igualdade e $\mathcal{K}_{i}$ é serial.

Agora podemos mostrar que:

\section{Teorema 3.13 (Satisfatibilidade em Estruturas Finitas).}

Todo $\varphi$ consistente em $\mathrm{K}_{n}$ (resp., $\mathrm{T}_{n}, \mathrm{~S}_{n}, \mathrm{~S}_{n}$ e $\mathrm{KD} 45_{n}$ ), é satisfativel em uma estrutura finita de $\mathcal{M}_{n}$ (resp., $\mathcal{M}_{n}^{r}, \mathcal{M}_{n}^{r t}, \mathcal{M}_{n}^{r s t}$ e $\mathcal{M}_{n}^{\text {elt }}$ ) com no máximo $2^{|\varphi|}$ mundos.

\section{Demonstração.}

Seguimos o esquema do Teorema 3.6 , porém utilizando a estrutura $\mathrm{M}_{\varphi}$ da Proposição 3.11 e para cada sistema adequamos as relações como descritas 
em 3.12. Assim, para mostrar que: toda fórmula consistente em $\mathrm{K}_{n}$ (resp., $\mathrm{T}_{n}, \mathrm{~S} 4_{n}, \mathrm{~S}_{n}$ e KD45 $)$ é satisfatível em alguma estrutura de $\mathcal{M}_{n}$ (resp., $\mathcal{M}_{n}^{r}$, $\mathcal{M}_{n}^{r t}, \mathcal{M}_{n}^{r s t}$ e $\left.\mathcal{M}_{n}^{\text {elt }}\right)$, basta considerarmos a estrutura $\mathrm{M}_{\varphi}$. Podemos tomar ( $\mathrm{M}_{\varphi}$, pois pela Proposição 3.12 se $\mathrm{M}_{\varphi}$ satisfaz os axiomas de $\mathrm{K}_{n}$ (resp., $\mathrm{T}_{n}$, $\mathrm{S} 4_{n}, \mathrm{~S} 5_{n}$ e $\mathrm{KD} 45_{n}$ ), então $\mathrm{M}_{\varphi}$ é elemento de $\mathcal{M}_{n}$ (resp., $\mathcal{M}_{n}^{r}, \mathcal{M}_{n}^{r t}, \mathcal{M}_{n}^{r s t}$ e $\left.\mathcal{M}_{n}^{\text {elt }}\right)$. Como na demonstração em 2.6, basta mostrar que $\left(\mathrm{M}_{\varphi}, w_{\Sigma}\right) \models \varphi$ se, e somente se, $\varphi \in \Sigma$, com $\Sigma \in \operatorname{Con}(\varphi)$. O esquema da demonstração, em todos os casos, é idêntico ao do Teorema 2.6.

Novamente, assim como em $\mathrm{K}_{n}$, temos que as propriedades operacionais de $\vdash \mathrm{e} \models$ são equivalentes nas extensões apresentadas, logo podemos tratar consistência e provabilidade em termos de satisfatibilidade e validade. Além disso, podemos, a partir da correção e completude em estruturas finitas, tratar a questão da decidibilidade (sec. 5.1) nos sistemas de conhecimento. Porém, antes de tratar esta questão vamos mostrar que é possível axiomatizar a noção de conhecimento comum e conhecimento distribuído. 


\section{Conhecimento Comum e Conhecimento Distribuído}

Dizemos que um fato (uma proposição $\varphi$ ) é de conhecimento mútuo entre as pessoas (agentes) de um determinado grupo se todos conhecem o fato. Se ocorrer uma divulgação aberta (por exemplo, um pronunciamento com todas as pessoas do grupo presentes como a feita pelo pai das crianças, no exemplo muddy children puzzle), o conhecimento mútuo torna-se conhecimento comum, ou seja, $\varphi$ é de conhecimento comum entre os agentes de um grupo se cada agente conhece $\varphi$, cada um deles sabe que todos conhecem $\varphi$, cada um deles sabe que todos sabem que conhecem $\varphi, \ldots$, ad infinitum.

John McCarthy em seu estudo sobre raciocínio baseado em bom-senso ${ }^{1}$ caracteriza o conhecimento comum como sendo aquilo que qualquer "tolo" sabe (qualquer tolo sabe o que é comumente conhecido por todos).

Do outro lado do espectro está o conceito de conhecimento distribuído que pode ser encarado como aquilo que um "sábio sabe" (alguém que tem o conhecimento daquilo que cada um do grupo sabe). Dizemos que os agentes de um grupo tem o conhecimento distribuído de um fato $\varphi$ se juntos podem inferir este fato (não é necessário o conhecimento mútuo de fatos que levem membros do grupo individualmente ao conhecimento de $\varphi$ ).

Este capítulo encontra-se dividido da seguinte forma: na primeira seção introduzimos os conceitos relativos a conhecimento comum e formalizamos as idéias envolvidas através da inclusão dos operadores $E_{G}$ e $C_{G}$ aos sistemas já estudados. Estudamos a correção e completude dos sistemas de conhecimento comum utilizando estruturas finitas. Na seção seguinte desen-

\footnotetext{
${ }^{1}$ Commom sense.
} 
volvemos os sistemas de conhecimento distribuído. Verificamos a correção e completude desses sistemas em estruturas não finitas.

\subsection{Conhecimento Comum}

Nesta seção estudamos o conhecimento comum como ferramenta de raciocínio entre agentes, conforme Halpern [36]. A formalização que damos, extraída do texto de Halpern, se verifica em sistemas síncronos ( $c f$. Lamport [49]), por exemplo, um sistema distribuído idealizado. Há o conceito de conhecimento comum concorrente (cf. Segerberg [73]) que é alcançável em ambientes assíncronos (cf. Panagadem e Taylor [64]), uma formalização axiomática foi feita por Costa em [16]. O estudo sobre o conceito de conhecimento comum concorrente está fora do escopo de nosso trabalho.

O estudo do conceito de conhecimento mútuo tem início com David Hume (1740), mas o primeiro exemplo de inferência baseado em conhecimento é devido a J. E. Littlewood (1953). Thomas Schelling (1960) e John Harsany (1967-68) analisaram situações em que o conhecimento comum é necessário para certos tipos de inferências que as pessoas fazem sobre outras. David Lewis (1969) foi o primeiro a apresentar uma análise explícita sobre conhecimento comum em sua monografia Convention: A Philosophical Study. Stephen Schiffer (1972) e Robert Aumann (1976) deram definições alternativas para conhecimento comum que em certos contextos são mais convenientes que a definição dada por Lewis. Aumann exibiu uma formulação matemática baseada na linguagem da teoria dos conjuntos, em termos de espaço de probabilidade, enquanto Schiffer fez uso do vocabulário da lógica epistêmica para definir conhecimento comum. A definição de Schiffer foi adotada por autores como Bacharach (1992), Bicchieri (1993) e Fagin, et al [24] (1995) para desenvolver teorias lógicas sobre conhecimento comum.

Para capturar a noção de conhecimento mútuo e conhecimento comum, entre os agentes de um grupo $G$, estendemos a linguagem $\mathrm{K}_{n}(\Phi)$ incluindo os operadores $E_{G}$ e $C_{G}$. Considere $G \subseteq \Delta$ não vazio e uma fórmula $\varphi$, então os novos operadores podem ser caracterizados do seguinte modo: $E_{G} \varphi$ diz que todos os agentes de $G$ conhecem $\varphi$, isto é, $E_{G} \varphi$ abrevia $\bigwedge_{i \in G} K_{i} \varphi$ e $C_{G} \varphi$ que diz que: $\varphi$ é de conhecimento dos agentes de $G$, que todos em $G$ sabem que todos conhecem $\varphi$, que todos em $G$ sabem que todos sabem que cada um deles conhece $\varphi$ etc, e é capturada como: $\bigwedge_{j \in \mathbb{N}^{*}} E_{G}^{j} \varphi, \operatorname{com} E_{G}^{1} \varphi=E_{G} \varphi \mathrm{e}$ $E_{G}^{j+1} \varphi=E_{G}\left(E_{G}^{j} \varphi\right), j \geq 1$.

Dizer que $E_{G} \varphi$ abrevia $\bigwedge_{i \in G} K_{i} \varphi$ equivale a afirmar que $E_{G} \varphi \rightarrow \bigwedge_{i \in G} K_{i} \varphi \mathrm{e}$ 
$\bigwedge_{i \in G} K_{i} \varphi \rightarrow E_{G} \varphi$, que denotamos por $E_{G} \varphi \leftrightarrow \bigwedge_{g \in G} K_{g} \varphi$. Para $|G|=1$ temos que $E_{G} \varphi$ equivale à $K_{i} \varphi$ para $i$ em $G$. E não podemos dizer que $C_{G} \varphi$ abrevia $\bigwedge_{j \in \mathbb{N}^{*}} E_{G}^{j} \varphi$ por esta ser uma conjunção infinitária. A semântica desses operadores é formalizada como segue:

Definição 4.1 .

$\left.1_{C}\right)(\mathrm{M}, w) \vDash E_{G} \varphi$ se e só se $(\mathrm{M}, w) \vDash K_{i} \varphi$, para todo $i \in G$,

$\left.2_{C}\right)(\mathrm{M}, w) \models C_{G} \varphi$ se e só se $(\mathrm{M}, w) \models E_{G}^{j} \varphi, j \in \mathbb{N}^{*}$.

As estruturas de Kripke quando representadas por meio de grafos ( $M$ é uma estrutura relacional) apresentam propriedades interessantes como a que definimos a seguir.

\section{Definição 4.2.}

Dado $G \subseteq \Delta$, dizemos que um mundo $w^{\prime}$ é G-atingivel a partir de um mundo $w$ em $m$ passos $(m \geq 1)$ se existem mundos $w_{0}, w_{1}, \ldots, w_{m}$ tais que $w_{0}=w, w_{m}=w^{\prime}$ e, para todo $j$ com $0 \leq j \leq m-1$, existe um agente $i$ em $G \operatorname{com}\left(w_{j}, w_{j+1}\right) \in \mathcal{K}_{i}$.

Para simplificar, dizemos que $w^{\prime}$ é G-atingivel a partir de $w$ se $w^{\prime}$ é $G$-atingivel a partir de $w$ em $m$ passos para algum $m \geq 1$. Na linguagem da teoria dos grafos dizer que $w^{\prime}$ é atingível a partir de $w$ em $m$ passos equivale à existência de um caminho de $w$ para $w^{\prime}$ de tamanho $m$; para as propriedades sobre grafos consultamos Diestel [18].

\section{Definição 4.3 .}

Seja $\Delta=\{1,2, \ldots, n\}$ e $\mathrm{M}=\left\langle W, \pi_{W}, \mathcal{K}_{1}, \ldots, \mathcal{K}_{n}\right\rangle$ com $s, t \in W$. Chamamos de caminho a seqüencia alternada de vértices (elementos de $W$ ) $e$ arestas (elementos de $\Delta$ ): $\left\langle v_{1}, i_{1}, v_{2}, i_{2}, \ldots, v_{m-1}, i_{m-1}, v_{m}\right\rangle$ com $v_{1}=s$, $v_{m}=t, v_{j} \in W(1 \leq j \leq m)$ e $i_{j}$ representando o par $\left(v_{j}, v_{j+1}\right) \in \mathcal{K}_{i_{j}}$ $(i \in \Delta$ e $1 \leq j<m)$. Chamamos tal seqüência de caminho de s para $t$ em $\mathrm{M}$.

As seguintes afirmações se verificam:

\section{Proposição 4.1.}

i) $(\mathrm{M}, w) \vDash E_{G}^{m} \varphi$ se e somente se $(\mathrm{M}, s) \vDash \varphi$ para todo $s$ G-atingivel a partir de $w$ em $m$ passos.

ii) $(\mathrm{M}, w) \models \mathrm{C}_{G} \varphi$ se e somente se $(\mathrm{M}, s) \models \varphi$ para todo $s$ G-atingivel a partir de $w$. 


\section{Demonstração.}

Considere $\mathrm{M}=\left\langle W, \pi_{W}, \mathcal{K}_{1}, \ldots, \mathcal{K}_{n}\right\rangle$ e $G \subseteq \Delta$ não vazio.

i) A demonstração segue por indução em $m$. Para $m=1,(\mathrm{M}, w) \models E_{G} \varphi$ se, e somente se, $(\mathrm{M}, w) \vDash K_{g} \varphi$, para todo $g \in G$. Isto é, para cada $g \in G$, temos que $(\mathrm{M}, s) \models \varphi$, para todo $s \in W$ tal que $(w, s) \in \mathcal{K}_{g}$. Logo, $(\mathrm{M}, s) \vDash \varphi$, para todo $s G$-atingível a partir de $w$ em 1 passo.

Como hipótese de indução temos: $(\mathrm{M}, w) \models E_{G}^{j} \psi$ se, e somente se, $(\mathrm{M}, s) \vDash \psi$, para todo $s G$-atingível a partir de $w$ em $j$ passos $(j \geq 1)$. Para mostrar que $(\mathrm{M}, w) \models E_{G}^{j+1} \varphi$ se, e só se, $(\mathrm{M}, s) \models \varphi$, para todo $s$ $G$-atingível a partir de $w$ em $j+1$ passos, basta tomar $\psi$ como sendo $E_{G} \varphi$. Da hipótese de indução, segue que $(\mathrm{M}, w) \models E_{G}^{j}\left(E_{G} \varphi\right)$ se, e só se, $(\mathrm{M}, s) \models E_{G} \varphi$, para todo $s G$-atingível a partir de $w$ em $j$ passos. Mas, $(\mathrm{M}, s) \vDash E_{G} \varphi$ se, e somente se, $(\mathrm{M}, s) \vDash K_{g} \varphi$, para todo $g \in G$, isto é, para cada $g \in G$, temos que $(M, t) \models \varphi$, para todo $t \in W$ tal que $(s, t) \in \mathcal{K}_{g}, \mathrm{e}(\mathrm{M}, t) \models \varphi$, para todo $t G$-atingível a partir de $w$ em $j+1$ passos. Portanto, segue a tese.

ii) $(\mathrm{M}, w) \models \mathrm{C}_{G} \varphi$ se, e só se, $(\mathrm{M}, w) \vDash E_{G}^{j} \varphi$, para todo $j \in \mathbb{N}^{*}$ que pelo item anterior ocorre se, e somente se, $(\mathrm{M}, s) \vDash \varphi$, para todo $s$ $G$-atingível a partir de $w$.

Da definição em 4.1 segue que $E_{G} \varphi$ é verdadeiro exatamente se a conjunção dos $K_{i} \varphi(i \in G)$ o é. $\mathrm{E}$ uma fórmula $\varphi$ é de conhecimento comum entre os elementos de um grupo $G$ exatamente se todos os elementos de $G$ sabem que $\varphi$ é verdadeiro e que $\varphi$ é de conhecimento comum.

\section{Observação 4.1 .}

$O$ operador $C_{G}$ preserva características do operador $K_{i}$, seguem dois exemplos:

- $\mathrm{M} \models C_{G}(\varphi \rightarrow \psi) \rightarrow\left(C_{G} \varphi \rightarrow C_{G} \psi\right)$.

De fato, de $(\mathrm{M}, w) \vDash C_{G}(\varphi \rightarrow \psi)$ e $(\mathrm{M}, w) \vDash C_{G} \varphi$ decorre que $(\mathrm{M}, s) \vDash \varphi \rightarrow \psi e(\mathrm{M}, s) \models \varphi$, para todo $s$ G-atingível a partir de w. Ou seja, $(\mathrm{M}, s) \models \psi$ (Teorema 2.4), para todo s G-atingível a partir de w. Então, $(\mathrm{M}, w) \vDash C_{G} \varphi$ e $\mathrm{M} \vDash C_{G}(\varphi \rightarrow \psi) \rightarrow\left(C_{G} \varphi \rightarrow C_{G} \psi\right)$.

- $\mathrm{M} \models\left(C_{G} \varphi \wedge C_{G} \psi\right) \leftrightarrow C_{G}(\varphi \wedge \psi)$.

$S e(\mathrm{M}, w) \vDash C_{G} \varphi \wedge C_{G} \psi$, então de $(\mathrm{M}, w) \vDash C_{G} \varphi$ e $(\mathrm{M}, w) \vDash C_{G} \psi$ decorre que $(\mathrm{M}, s) \models \varphi e(\mathrm{M}, s) \models \psi$, para todo $s$-atingivel a partir de w. Logo, $(\mathrm{M}, s) \vDash \varphi \wedge \psi$ e (Observação 2.2) $e(\mathrm{M}, w) \vDash C_{G}(\varphi \wedge \psi)$. Por outro lado, se $(\mathrm{M}, w) \vDash C_{G}(\varphi \wedge \psi)$ tem-se que $(\mathrm{M}, s) \models \varphi \wedge$ 
$\psi$, para todo $s$ G-atingivel a partir de w. Ou seja, $(\mathrm{M}, s) \models \varphi e$ $(\mathrm{M}, s) \vDash \psi$ (Observação 2.2), logo $(\mathrm{M}, w) \vDash C_{G} \varphi$ e $(\mathrm{M}, w) \vDash C_{G} \psi$, então $(\mathrm{M}, w) \vDash C_{G} \varphi \wedge C_{G} \psi$. Portanto, $\mathrm{M} \vDash\left(C_{G} \varphi \wedge C_{G} \psi\right) \leftrightarrow C_{G}(\varphi \wedge$ $\psi)$.

$C_{G} \varphi$ pode ser visto como ponto fixo da função $f(x)=E_{G}(\varphi \wedge x)$. Este modo de tratar o operador $C_{G}$ pode ser encontrado em [35] e [24, pág. 402-411].

A seguir formalizamos a relação entre conhecimento mútuo e conhecimento comum, assim como uma forma para tratar a dedução envolvendo o conhecimento comum.

\section{Teorema 4.2.}

Para quaisquer fórmulas $\varphi, \psi \in \mathrm{K}_{n}^{C}(\Phi)$, estrutura $\mathrm{M}$ de $\mathcal{M}_{n}$ e $G \subseteq \Delta$ não vazio temos que:

i) $\mathrm{M} \models C_{G} \varphi \leftrightarrow E_{G}\left(\varphi \wedge C_{G} \varphi\right)$,

ii) se $\mathrm{M} \vDash \varphi \rightarrow E_{G}(\psi \wedge \varphi)$, então $\mathrm{M} \vDash \varphi \rightarrow C_{G} \varphi$.

Demonstração. [Halpern [36]]

i) Vamos mostrar que $\mathrm{M} \models C_{G} \varphi \rightarrow E_{G}\left(\varphi \wedge C_{G} \varphi\right)$. Suponha por absurdo que $\mathrm{M} \not \models C_{G} \varphi \rightarrow E_{G}\left(\varphi \wedge C_{G} \varphi\right)$, isto é, existe um mundo $w$ tal que $(\mathrm{M}, w) \vDash C_{G} \varphi$ e $(\mathrm{M}, w) \not E_{G}\left(\varphi \wedge C_{G} \varphi\right)$. De $(\mathrm{M}, w) \vDash C_{G} \varphi$ temos que para todo $s$-atingível a partir de $w(\mathrm{M}, w) \vDash \varphi$. Por outro lado, $(\mathrm{M}, w) \not \models E_{G}\left(\varphi \wedge C_{G} \varphi\right)$ contradiz o fato anterior, pois tem-se que $(\mathrm{M}, w) \not \models K_{g}\left(\varphi \wedge C_{G} \varphi\right)$ para algum $g$ de $G$, isto é, existe $t$ tal que $(w, t) \in \mathcal{K}_{g}$ e $(\mathrm{M}, t) \not \models \varphi$ ou $(\mathrm{M}, t) \not \models C_{G} \varphi$. Ou seja, $t$ é $G$-atingível a partir de $w \operatorname{com}(\mathrm{M}, t) \not \models \varphi$ e existe $t^{\prime} G$-atingível a partir de $t$, portanto $G$-atingivel a partir de $w \operatorname{com}\left(\mathrm{M}, t^{\prime}\right) \not \models \varphi$.

A recíproca, $\mathrm{M} \vDash E_{G}\left(\varphi \wedge C_{G} \varphi\right) \rightarrow C_{G} \varphi$, segue da suposição de que $\mathrm{M} \not \models E_{G}\left(\varphi \wedge C_{G} \varphi\right) \rightarrow C_{G} \varphi$. Logo, $(\mathrm{M}, w) \vDash E_{G}\left(\varphi \wedge C_{G} \varphi\right)$ e $(\mathrm{M}, w) \not C_{G} \varphi$ para algum $w$. De $(\mathrm{M}, w) \vDash E_{G}\left(\varphi \wedge C_{G} \varphi\right)$ tem-se que $(\mathrm{M}, s) \vDash \varphi$ e $(\mathrm{M}, s) \vDash C_{G} \varphi$, para todo $s G$-atingível a partir de $w$ tal que $(w, s) \in \mathcal{K}_{i}$, o que contradiz $(\mathrm{M}, w) \not C_{G} \varphi$. Portanto, vale a equivalência.

ii) Neste caso, basta verificarmos que para cada mundo $w$ de $\mathrm{M}$ ou temos $(\mathrm{M}, w) \not \models \varphi$ ou $(\mathrm{M}, w) \vDash C_{G} \psi$. Como supomos $\mathrm{M} \models \varphi \rightarrow E_{G}(\psi \wedge \varphi)$, então basta que para cada $s$ de $M G$-atingível a partir de $w$ em $m$ passos $(m \geq 1)$ tenhamos $(M, s) \vDash \psi$. A demonstração segue por indução em $m$. 
Base: como $M \vDash \varphi \rightarrow E_{G}(\psi \wedge \varphi)$ e para todo $w$ vale $(\mathrm{M}, w) \models \varphi$, temos que $(\mathrm{M}, w) \models \varphi \rightarrow E_{G}(\psi \wedge \varphi)$, isto é, $(\mathrm{M}, w) \models K_{i}(\psi \wedge \varphi)$, para todo $i$ em $G$. Logo, para cada $i$ em $G$ e para todo $s$ tal que $(w, s) \in \mathcal{K}_{i}$ vale que $(\mathrm{M}, s) \models \psi \wedge \varphi$. Ou seja, $(\mathrm{M}, s) \models \psi$, para todo $s G$-atingível a partir de $w$ em um passo.

Passo Indutivo: supondo que a afirmação valha para $j(j \geq 1)$ passos, mostramos que $(\mathrm{M}, s) \models \psi$ para todo $s G$-atingível a partir de $w$ em $j+1$ passos. Seja $t G$-atingível a partir de $w$ em $j$ passos, então de $\mathrm{M} \vDash \varphi \rightarrow E_{G}(\psi \wedge \varphi)$ e de que para todo $w(\mathrm{M}, w) \vDash \varphi$, temos em particular $(\mathrm{M}, t) \models K_{i}(\psi \wedge \varphi)$, para todo $i$ em $G$, Logo, para cada $i$ de $G$ e para todo $s$ tal que $(t, s) \in \mathcal{K}_{i}$ vale que $(\mathrm{M}, s) \models \psi \wedge \varphi$. Ou seja, $(\mathrm{M}, t) \models \psi$ para todo $t G$-atingível a partir de $w$ em $j+1$ passos. Portanto, segue a tese.

As propriedades descritas no teorema anterior caracterizam $C_{G}$ tornando-o operacional, apesar de ser um "operador infinitário", além disso estas propriedades permitem adotarmos os seguintes axiomas (cf., Milgrom [58] e Halpern [36]) para definir os sistemas $\mathrm{K}_{n}^{C}, \mathrm{~T}_{n}^{C}, \mathrm{~S} 4_{n}^{C}, \mathrm{~S} 5_{n}^{C}$ e $\mathrm{KD} 45_{n}^{C}$.

\section{Definição 4.4.}

Definimos $\mathrm{K}_{n}^{C}$ (resp., $\mathrm{T}_{n}^{C}, \mathrm{~S} 4_{n}^{C}, \mathrm{~S}_{n}^{C}$ e $\mathrm{KD}_{4} 5_{n}^{C}$ ), como extensão de $\mathrm{K}_{n}$ (resp., $\mathrm{T}_{n}, \mathrm{~S}_{n}, \mathrm{~S}_{n}$ e $\mathrm{KD} 5_{n}$ ), com a inclusão dos seguintes axiomas:

$\left.1_{K_{n}^{C}}\right) E_{G} \varphi \leftrightarrow \bigwedge_{i \in G} K_{i} \varphi \quad$ (axioma do conhecimento mútuo),

$\left.2_{K_{n}^{C}}\right) C_{G} \varphi \rightarrow E_{G}\left(\varphi \wedge C_{G} \varphi\right) \quad$ (axioma do ponto-fixo),

e da seguinte regra $(R I)$ :

$$
\frac{\vdash_{K_{n}^{C}} \varphi \rightarrow E_{G}(\psi \wedge \varphi)}{\vdash_{K_{n}^{C}} \varphi \rightarrow C_{G} \psi} \quad \text { (regra de indução) }
$$

Os nomes atribuídos aos axiomas são de acordo com o que foi exposto anteriormente, já a chamada regra de indução tem este nome por ser essencial em sua demonstração o uso do princípio de indução finita, é feita a indução sobre $j$ para mostrar que $\varphi \rightarrow E_{G}^{j}(\psi \wedge \varphi)$ é válida para todo $j$. Os teoremas 4.3 e 4.8 mostram que os novos sistemas caracterizam formalmente a noção de conhecimento comum. 
Teorema 4.3.

O sistema $\mathrm{K}_{n}^{C}$ (resp., $\mathrm{T}_{n}^{C}, \mathrm{~S} 4_{n}^{C}, \mathrm{~S}_{n}^{C}$ e $\mathrm{KD} 45_{n}^{C}$ ) é correto em relação a $\mathcal{M}_{n}$ (resp., $\mathcal{M}_{n}^{r}, \mathcal{M}_{n}^{r t}, \mathcal{M}_{n}^{r s t}, \mathcal{M}_{n}^{\text {elt }}$ ).

\section{Demonstração.}

Do Teorema 4.2 temos a validade dos axiomas e que $R I$ preserva a validade em relação à $\mathcal{M}_{n}$ (resp., $\left.\mathcal{M}_{n}^{r}, \mathcal{M}_{n}^{r t}, \mathcal{M}_{n}^{r s t}, \mathcal{M}_{n}^{\text {elt }}\right)$. Logo, a correção de $\mathrm{K}_{n}^{C}$ (resp., $\mathrm{T}_{n}^{C}, \mathrm{~S} 4_{n}^{C}, \mathrm{~S} 5_{n}^{C}$ e $\mathrm{KD} 45_{n}^{C}$ ) segue de modo idêntico aos teoremas 2.5 e 3.5 .

Antes de demonstrarmos a completude para os sistemas de conhecimento comum (Teorema 4.8) estudamos o comportamento do operador $C_{G}$ em estruturas de Kripke finitas. A construção dessas estruturas é baseada nas estruturas $M_{\varphi}$ e para isso necessitamos estender os conjuntos definidos em 3.8.

Definição 4.5.

Dada uma fórmula $\varphi$ denotamos:

- $S u b_{C}(\varphi)$ a união dos conjuntos:

$-S u b(\varphi)$

$-\left\{\psi \wedge C_{G} \psi: C_{G} \psi \in S u b(\varphi)\right\}$

- $\left\{E_{G}\left(\psi \wedge C_{G} \psi\right): C_{G} \psi \in S u b(\varphi)\right\}$

- $\left\{K_{i}\left(\psi \wedge C_{G} \psi\right): C_{G} \psi \in S u b(\varphi)\right.$ e $\left.i \in G\right\}$

- $\left\{K_{i} \psi: E_{G} \psi \in \operatorname{Sub}(\varphi)\right.$ e $\left.i \in G\right\}$,

- $S u b_{C}(\varphi)$ o conjunto formado pelos elementos de $S u b_{C}(\varphi)$ e suas respectivas negações,

- $\operatorname{Con}_{C}(\varphi)$ o conjunto de todos os subconjuntos consistentes e maximais em relação a $S u b_{C}(\varphi)$.

Veja que a forma das fórmulas que compõem o conjunto $S u b_{C}(\varphi)$ visa contemplar os axiomas do conhecimento mútuo e do ponto-fixo, em relação às subfórmulas de $\varphi$. Os conjuntos acima descritos são finitos:

\section{Proposição 4.4.}

Dada uma fórmula $\varphi$ temos que:

i) todo subconjunto consistente de $\operatorname{Sub} b_{C}(\varphi)$ pode ser estendido a um elemento de $\operatorname{Con}_{C}(\varphi)$, 
ii) se $\Sigma^{\prime} \in \operatorname{Con}_{C}(\varphi)$, então para qualquer $\psi$ de $S u b_{C}(\varphi)$, temos que $\Sigma^{\prime}$ contém $\psi$ ou $\neg \psi$, mas não ambos,

iii) $\left|S u b_{C}(\varphi)\right| \leq(n+3)|\varphi|$.

\section{Demonstração.}

As demonstrações dos primeiros dois itens é imediata. A última afirmação segue dos seguintes fatos:

- $|\operatorname{Sub}(\varphi)| \leq|\varphi|$.

- $\left|\left\{\psi \wedge C_{G} \psi: C_{G} \psi \in S u b(\varphi)\right\}\right| \leq|\varphi|$.

- $\left|\left\{E_{G}\left(\psi \wedge C_{G} \psi\right): C_{G} \psi \in \operatorname{Sub}(\varphi)\right\}\right| \leq|\varphi|$.

- $\mid\left\{K_{i}\left(\psi \wedge C_{G} \psi\right): C_{G} \psi \in S u b(\varphi)\right.$ e $\left.i \in G\right\}\left|\leq\left\lceil\frac{n}{2}\right\rceil\right| \varphi \mid$

- $\mid\left\{K_{i} \psi: E_{G} \psi \in S u b(\varphi)\right.$ e $\left.i \in G\right\}\left|\leq\left\lceil\frac{n}{2}\right\rceil\right| \varphi \mid$.

Logo, $\left|S u b_{C}(\varphi)\right| \leq(n+3)|\varphi|$.

A finitude de $\operatorname{Con}_{C}(\varphi)$ permite-nos construir fórmulas a partir $S u b_{C}(\varphi)$. Dado $\Gamma \in \operatorname{Con}_{C}(\varphi)$, denotamos por $\varphi_{\Gamma}$ a conjunção de todas as fórmulas em $\Gamma$ e, como $\Gamma$ é finito, se $\varphi \in K_{n}^{C}(\Phi)$, então $\varphi_{\Gamma} \in K_{n}^{C}(\Phi)$. Do mesmo modo, a expressão $\underset{\Gamma \in C o n_{C}(\varphi)}{\bigvee} \varphi_{\Gamma}$ é fórmula de $K_{n}^{C}(\Phi)$.

\section{Proposição 4.5.}

Se $\varphi$ é fórmula de $\mathrm{K}_{n}^{C}$, então $\vdash_{K_{n}^{C}} \underset{\Gamma \in C o n_{C}(\varphi)}{\bigvee} \varphi_{\Gamma}$.

\section{Demonstração.}

Suponha que $\nvdash_{K_{n}^{C}} \underset{\Gamma \in C \text { on }_{C}(\varphi)}{\bigvee} \varphi_{\Gamma}$, então $\left\{\bigwedge_{\Gamma \in \operatorname{Con}_{C}(\varphi)}\left(\neg \varphi_{\Gamma}\right)\right\}$ é $\kappa_{n}^{C}$-consistente.

Pelo lema de Lindenbaun, seja $\Sigma$ sua extensão maximal $\kappa_{n}^{C}$-consistente. Veja que $\neg \varphi_{\Gamma} \in \Sigma$ para cada $\Gamma$ de $\operatorname{Con}_{C}(\varphi)$ (item (iii) da Prop. 2.3). Por outro lado, seja $\Sigma^{\prime}=\Sigma \cap S u b_{C}^{\neg}(\varphi)$, então $\Sigma^{\prime} \in C o n_{C}(\varphi)$ (item (ii) da Prop. 3.10). Então, $\varphi_{\Sigma^{\prime}} \in \Sigma\left(\Sigma^{\prime} \subseteq \Sigma\right)$, o que contradiz o fato de $\bigwedge_{\Gamma \in C o n_{C}(\varphi)}\left(\neg \varphi_{\Gamma}\right) \in \Sigma$.

As estruturas $\mathrm{M}_{\varphi}$ são baseadas em $\operatorname{Con}{ }_{C}(\varphi)$ e a leitura que fazemos de $\mathrm{M}_{\varphi}$ é como segue: dado $\Gamma$, o mundo $w_{\Gamma}$ em $\mathrm{M}_{\varphi}$ é completamente descrito pela conjunção de todas as fórmulas de $\Gamma$, portanto se a quantidade de mundos em $\mathrm{M}_{\varphi}$ é finita podemos descrever todos os mundos pela disjunção das fórmulas $\varphi_{\Gamma}$, para cada $\Gamma$ que determina um mundo em $M_{\varphi}$. Como desejamos tratar o conhecimento comum $\mathrm{M}_{\varphi}$, deve conter somente os mundos $w_{\Gamma}$ onde $C_{G} \psi$ é 
satisfeita. Portanto, $\mathbf{M}_{\varphi}$ fica perfeitamente descrita por $\varphi_{\Psi} \operatorname{com} \varphi_{\Psi}=\bigvee_{\Gamma \in \Psi} \varphi_{\Gamma}$ e $\Psi=\left\{\Gamma \in \operatorname{Con}_{C}(\varphi):\left(\mathrm{M}_{\varphi}, w_{\Gamma}\right) \models C_{G} \psi\right\}$. A proposição a seguir formaliza a situação descrita.

\section{Proposição 4.6 (Conhecimento Comum e Estruturas Finitas).}

Dada uma fórmula $\varphi$ seja $\mathrm{M}_{\varphi}=\left\langle W, \pi_{W}, \mathcal{K}_{1}, \ldots, \mathcal{K}_{n}\right\rangle$ a estrutura definida como segue: $W=\left\{w_{\Gamma} \mid \Gamma \in \operatorname{Con}_{C}(\varphi)\right\}$.

Para $\chi \in \Phi: \quad \pi_{w_{\Gamma}}(\chi)= \begin{cases}\top & \text { se } \chi \in \Gamma \\ \perp & \text { se } \chi \notin \Gamma\end{cases}$

Seja $\Gamma / K_{i}=\left\{\chi \mid K_{i} \chi \in \Gamma\right\}$, então $\mathcal{K}_{i}=\left\{\left(w_{\Gamma}, w_{\Gamma^{\prime}}\right) \mid \Gamma / K_{i} \subseteq \Gamma^{\prime}\right\}$.

Então, $\mathrm{M}_{\varphi}$ é finita e $\vdash_{\mathrm{K}_{n}^{C}} \varphi_{\Psi} \rightarrow C_{G} \psi$.

Demonstração. [Halpern [36]]

A finitude de $\mathrm{M}_{\varphi}$ decorre de forma imediata da Proposição 4.4. Para mostrar que $\vdash_{K_{n}^{C}} \varphi_{\Psi} \rightarrow C_{G} \psi$, basta verificar que $\vdash_{K_{n}^{C}} \varphi_{\Psi} \rightarrow E_{G}\left(\psi \wedge \varphi_{\Psi}\right)$, pois por $R I$ segue a tese. Para a demonstração de $\vdash_{K_{n}^{C}} \varphi_{\Psi} \rightarrow E_{G}\left(\psi \wedge \varphi_{\Psi}\right)$, considere $\bar{\Psi}=\operatorname{Con}_{C}(\varphi)-\Psi$. Mostramos que para quaisquer $i$ de $G, \Gamma \in \Psi$ e $\Sigma \in \bar{\Psi}$ são teoremas de $K_{n}^{C}$ :

a) $\vdash_{K_{n}^{C}} \varphi_{\Gamma} \rightarrow K_{i}\left(\neg \varphi_{\Sigma}\right)$,

b) $\vdash_{K_{n}^{C}} \varphi_{\Gamma} \rightarrow K_{i} \psi$

c) $\vdash_{K_{n}^{C}} \varphi_{\Psi} \leftrightarrow \neg\left(\bigvee_{\Sigma \in \bar{\Psi}} \varphi_{\Sigma}\right)$

A partir desses teoremas, fixados $i$ em $G$ e $\Gamma$ em $\Psi$, decorre de $(a)$ que $\vdash_{K_{n}^{C}} \varphi_{\Gamma} \rightarrow \bigwedge_{\Sigma \in \bar{\Psi}} K_{i}\left(\neg \varphi_{\Sigma}\right)$ e pelo item (i) da Proposição 2.1 juntamente com $S H$ temos $\vdash_{K_{n}^{C}} \varphi_{\Gamma} \rightarrow K_{i}\left(\bigwedge \neg \varphi_{\Sigma}\right)$, para quaisquer $i$ de $G$ e $\Gamma$ em $\Psi$. Por $(b) \vdash_{K_{n}^{C}} \varphi_{\Gamma} \rightarrow\left(K_{i} \psi \wedge K_{i}\left(\bigwedge_{\Sigma \in \bar{\Psi}}^{\Sigma \in \bar{\Psi}} \neg \varphi_{\Sigma}\right)\right) \mathrm{e} \vdash_{K_{n}^{C}} \varphi_{\Gamma} \rightarrow K_{i}\left(\psi \wedge\left(\bigwedge_{\Sigma \in \bar{\Psi}} \neg \varphi_{\Sigma}\right)\right)$, para quaisquer $i$ de $G$ e $\Gamma$ em $\Psi$. De $(c)$ temos $\vdash_{K_{n}^{C}} \varphi_{\Gamma} \rightarrow K_{i}\left(\psi \wedge \varphi_{\Psi}\right)$ para cada $i$ de $G$ e $\Gamma$ em $\Psi$, ou seja, $\vdash_{K_{n}^{C}} \varphi_{\Gamma} \rightarrow \bigwedge_{i \in G} K_{i}\left(\psi \wedge \varphi_{\Psi}\right)$ para qualquer $\Gamma$ em $\Psi$. Logo, pelo axioma do conhecimento mútuo $\vdash_{K_{n}^{C}} \varphi_{\Gamma} \rightarrow E_{G}\left(\psi \wedge \varphi_{\Psi}\right)$, para todo $\Gamma$ em $\Psi$. Em particular, $\vdash_{K_{n}^{C}} \varphi_{\Psi} \rightarrow E_{G}\left(\psi \wedge \varphi_{\Psi}\right)$.

A seguir a demonstração de cada um dos teoremas afirmados.

a) A demonstração segue da suposição de que se $\forall_{\kappa_{n}^{C}} \varphi_{\Gamma} \rightarrow K_{i}\left(\neg \varphi_{\Sigma}\right)$, então $\left(w_{\Gamma}, w_{\Sigma}\right) \in \mathcal{K}_{i}$, o que leva a contradizer o fato de $\Gamma$ pertencer a $\Psi$. 
Como $\Sigma \in \bar{\Psi}$, então $\left(\mathrm{M}_{\varphi}, w_{\Sigma}\right) \not \models C_{G} \psi$, logo existe $w_{\Sigma^{\prime}}$ em $\mathrm{M}_{\varphi} G^{-}$ atingível a partir de $w_{\Sigma}$ tal que $\left(\mathrm{M}_{\varphi}, w_{\Sigma^{\prime}}\right) \not \neq \psi$. Portanto, se $\left(w_{\Gamma}, w_{\Sigma}\right) \in$ $\mathcal{K}_{i}$, temos que $\left(\mathrm{M}_{\varphi}, w_{\Gamma}\right) \not C_{G} \psi$ e $\Gamma \in \bar{\Psi}$.

Resta mostrar que se $\nvdash_{K_{n}^{C}} \varphi_{\Gamma} \rightarrow K_{i}\left(\neg \varphi_{\Sigma}\right)$, então $\left(w_{\Gamma}, w_{\Sigma}\right) \in \mathcal{K}_{i}$, isto é, que $\Gamma / K_{i} \subseteq \Sigma$, para cada $i$ de $G$. De modo mais geral, vamos mostrar que $\Gamma / K_{i} \cup \Gamma / E_{G} \subseteq \Sigma$, para $\Gamma / E_{G}=\left\{\chi \mid E_{G} \chi \in \Gamma\right\}$. Suponha que não, que $\Gamma / K_{i} \cup \Gamma / E_{G} \nsubseteq \Sigma$, então, existem $i$ em $G$ e $\chi$ em $S u b_{C}(\varphi)$ tais que $E_{G} \chi \in \Gamma$ e $\chi \notin \Sigma$. Veja que isto implica em $\Gamma / K_{i} \nsubseteq \Sigma$ e $\neg \chi \in \Sigma$. Como $\vdash_{K_{n}^{C}} \chi \rightarrow\left(\chi^{\prime} \rightarrow \chi\right)$ e $\neg \varphi_{\Sigma}$ abrevia $\bigvee_{\varsigma \in \Sigma} \neg \varsigma_{\Sigma} \vee \chi$ para $\varsigma \neq \neg \chi$, então $\vdash_{K_{n}^{C}} \chi \rightarrow \neg \varphi_{\Sigma}$. Aplicando, na seqüência, $G M$, o axioma $K$ e $M P$ obtemos $\vdash_{K_{n}^{C}} K_{i} \chi \rightarrow K_{i}\left(\neg \varphi_{\Sigma}\right)$, para todo $i$ de $G$. Ainda, $\vdash_{K_{n}^{C}} E_{G} \chi \rightarrow K_{i} \chi$ para cada $i$ de $G$ (item (2) da Observação 1.2) e $\vdash_{K_{n}^{C}} \varphi_{\Gamma} \rightarrow E_{G} \chi,\left(E_{G} \chi \in \Gamma\right.$ e item (2) da Observação 1.2), então $\vdash_{K_{n}^{C}} \varphi_{\Gamma} \rightarrow K_{i}\left(\neg \varphi_{\Sigma}\right)$. Logo, segue a tese.

b) A demonstração é análoga ao item anterior, suponha que $\left\{\varphi_{\Gamma} \wedge \neg K_{i} \psi\right\}$ é $\kappa_{n}^{C}$-consistente. Então, existe $\Sigma^{\prime} \in \operatorname{Con}_{C}(\varphi)$ tal que $\left(w_{\Gamma}, w_{\Sigma^{\prime}}\right) \in \mathcal{K}_{i}$ e $\left(\mathrm{M}_{\varphi}, w_{\Sigma^{\prime}}\right) \not \models \psi$. Para $\Sigma^{\prime}$ basta considerar que existe (lema de Lindenbaun) $\Sigma$ extensão maximal $\kappa_{n}^{C}$-consistente de $\left\{\varphi_{\Gamma} \wedge \neg K_{i} \psi\right\}$ e $\Sigma^{\prime}=\Sigma \cap S u b_{C}^{ᄀ}(\varphi)$, então $\Sigma^{\prime} \in \operatorname{Con}_{C}(\varphi)$ e maximal em $S u b_{C}^{\neg}(\varphi)$. No entanto, a existência de $\Sigma^{\prime}$ contradiz o fato de $\left(\mathrm{M}_{\varphi}, w_{\Gamma}\right) \models C_{G} \psi$.

c) Para verificar $\vdash_{K_{n}^{C}} \varphi_{\Psi} \leftrightarrow \bigwedge_{\Sigma \in \bar{\Psi}} \neg \varphi_{\Sigma}$ basta observar que na Proposição 4.5 as fórmulas $\varphi_{\Gamma}$ são mutuamente exclusivas.

Novamente, observamos que para qualquer fórmula $\varphi$ se $\psi \in \Phi$ e $\psi \notin S u b(\varphi)$, então $\psi$ é falso em qualquer mundo de $\mathrm{M}_{\varphi}$. Outra propriedade de $\mathrm{M}_{\varphi}$ é dada pelo teorema a seguir.

\section{Teorema 4.7 (Satisfatibilidade em Estruturas Finitas).}

Toda fórmula $\varphi$ consistente em $\mathrm{K}_{n}^{C}$ (resp., $\mathrm{T}_{n}^{C}, \mathrm{~S}_{n}^{C}, \mathrm{~S}_{n}^{C}$ e $\mathrm{KD}_{4} 5_{n}^{C}$ ) é satisfativel em alguma estrutura de $\mathcal{M}_{n}$ (resp., $\mathcal{M}_{n}^{r}, \mathcal{M}_{n}^{r t}, \mathcal{M}_{n}^{r s t}$ e $\mathcal{M}_{n}^{\text {elt }}$ ).

Demonstração. [Halpern [36]]

Novamente, o esquema é mostrar que se $\varphi$ é consistente em $\mathrm{K}_{n}^{C}$ (resp., $\mathrm{T}_{n}^{C}, \mathrm{~S} 4_{n}^{C}, \mathrm{~S} 5_{n}^{C}$ e $\mathrm{KD} 45_{n}^{C}$ ), então para qualquer $\psi$ em $S u b_{C}^{\sim}(\varphi)$ tem-se que $\left(\mathrm{M}_{\varphi}, w_{\Gamma}\right) \vDash \psi$ se, e somente se, $\psi \in \Gamma$. A estrutura $\mathrm{M}_{\varphi}$ é o da Proposição 3.11 .

Como antes, a demonstração é por indução no número de conectivos que ocorrem em $\varphi$, a argumentação para os casos em que $\psi$ é uma proposição primitiva, tem forma de negação, implicação ou $K_{i} \chi$ é idêntica àquela feita no Teorema 2.6. Resta verificarmos para $\psi$ da forma $E_{G} \chi$ e $C_{G} \chi$. 
- $\psi$ é da forma $E_{G} \chi$. Para demonstrar que $E_{G} \chi \in \Gamma$ se, e só se, $\left(\mathrm{M}_{\varphi}, w_{\Gamma}\right) \vDash E_{G} \chi$, estamos supondo demonstrado que $K_{i} \chi \in \Gamma$ se, e só se, $\left(\mathrm{M}_{\varphi}, w_{\Gamma}\right) \models K_{i} \chi$ para todo $i$ de $G$. Temos que $E_{G} \chi \in \Gamma$ se, e só se, $K_{i} \chi \in \Gamma$ para todo $i$ de $G$, que decorre da definição de $S u b_{C}(\varphi)$, de $\Gamma \in \operatorname{Con}_{C}(\varphi)$ e do axioma do conhecimento mútuo. Então, $\left(\mathrm{M}_{\varphi}, w_{\Gamma}\right) \models \bigwedge_{i \in G} K_{i} \chi$, que equivale a $\left(\mathrm{M}_{\varphi}, w_{\Gamma}\right) \models E_{G} \chi$ (axioma do conhecimento mútuo).

- $\psi$ da forma $C_{G} \chi$. Para a demonstração de que se $C_{G} \chi \in \Gamma$ então $\left(\mathrm{M}_{\varphi}, w_{\Gamma}\right) \vDash C_{G} \chi$, nenhum ferramental extra é utilizado, fazemos uso do princípio de indução finita, porém, para a recíproca, necessitamos dos resultados da Proposição 4.6 .

Iniciamos com: se $C_{G} \chi \in \Gamma$, então $\left(\mathrm{M}_{\varphi}, w_{\Gamma}\right) \models C_{G} \chi$. Pela Proposição 4.1, basta verificarmos que $\left(\mathrm{M}_{\varphi}, w_{\Sigma}\right) \models \chi$, para todo $w_{\Sigma} G$-atingível a partir de $w_{\Gamma}$. A demonstração segue por indução no número de passos $m$ para $G$-atingir $w_{\Sigma}$ a partir de $w_{\Gamma}$.

Base: $\operatorname{De} C_{G} \chi \in \Gamma, \Gamma \in \operatorname{Con}_{C}(\varphi)$ e $\vdash_{\mathrm{K}_{n}^{C}} C_{G} \chi \rightarrow E_{G}\left(\chi \wedge C_{G} \chi\right)$ decorre que $E_{G}\left(\chi \wedge C_{G} \chi\right) \in \Gamma$ e, como no item anterior, $K_{i}\left(\chi \wedge C_{G} \chi\right) \in \Gamma$, com $\left(\mathrm{M}_{\varphi}, w_{\Gamma}\right) \models K_{i}\left(\chi \wedge C_{G} \chi\right)$, para todo $i$ de $G$, ou seja, temos que $\left(\mathrm{M}_{\varphi}, w_{\Sigma}\right) \vDash \chi \wedge C_{G} \chi$, para todo $w_{\Sigma}$ de $W_{\varphi}$, tal que $\left(w_{\Gamma}, w_{\Sigma}\right) \in \mathcal{K}_{i}$. Em particular, $\left(\mathrm{M}_{\varphi}, w_{\Sigma}\right) \models \chi$, para qualquer mundo $w_{\Sigma} G$-atingível a partir de $w_{\Gamma}$ em um passo.

Passo Indutivo: Suponha que $\left(\mathrm{M}_{\varphi}, w_{\Sigma}\right) \vDash \chi$, para todo $w_{\Sigma} \in W_{\varphi}$ $G$-atingivel a partir de $w_{\Gamma}$ em $m$ passos. Seja $w_{\Sigma^{\prime \prime}} \in W_{\varphi} G$-atingível a partir de $w_{\mathrm{r}}$ em $m+1$ passos. Então existe $w_{\Sigma^{\prime}} \in W_{\varphi} G$-atingível a partir de $w_{\Gamma}$ em $m$ passos com $\left(w_{\Sigma^{\prime}}, w_{\Sigma^{\prime \prime}}\right) \in \mathcal{K}_{i}$. Pela hipótese de indução $\left(\mathrm{M}_{\varphi}, w_{\Sigma^{\prime}}\right) \vDash \chi \wedge C_{G} \chi$, isto é, $\chi$ e $C_{G} \chi$ são elementos de $\Sigma^{\prime}$, então $\left(\mathrm{M}_{\varphi}, w_{\Sigma^{\prime \prime}}\right) \vDash \chi$, a demonstração é análoga à da base. Logo, pelo princípio de indução finita, $\left(\mathrm{M}_{\varphi}, w_{\Sigma}\right) \models \chi$, para todo $w_{\Sigma}$ em $\mathrm{M}_{\varphi}$ $G$-atingível a partir de $w_{\Gamma}$. Portanto, $\left(\mathrm{M}_{\varphi}, w_{\Gamma}\right) \models C_{G} \chi$.

A recíproca, se $\left(\mathrm{M}_{\varphi}, w_{\Gamma}\right) \vDash C_{G} \psi$, então $C_{G} \psi \in \Gamma$. Suponha que $C_{G} \psi \notin \Gamma$, logo $\neg C_{G} \psi \in \Gamma$. Por outro lado, como $\left(\mathrm{M}_{\varphi}, w_{\Gamma}\right) \models C_{G} \psi$ e considerando o conjunto $\Psi$ da Proposição 4.6, temos que $\Gamma \in \Psi$ e $\vdash_{K_{n}^{C}} \varphi_{\Gamma} \rightarrow \varphi_{\Psi}$. Como $\vdash_{K_{n}^{C}} \varphi_{\Psi} \rightarrow C_{G} \psi$ (Prop. 4.6), então, aplicando $S H$, obtemos $\vdash_{K_{n}^{C}} \varphi_{\Gamma} \rightarrow C_{G} \psi$. Logo, $\neg \varphi_{\Gamma} \in \Gamma$ e $\Gamma$ não é $K_{n}^{C}$-consistente (contradição!). Portanto, $C_{G} \psi \in \Gamma$.

Então, toda fórmula $\varphi \mathrm{K}_{n}^{C}$-consistente é satisfatível em $\mathrm{M}_{\varphi}$ de $\mathcal{M}_{n}$. Os outros casos seguem de modo idêntico.

E segue a completude dos sistemas de conhecimento comum: 


\section{Teorema 4.8.}

$\mathrm{K}_{n}^{C}$ (resp., $\mathrm{T}_{n}^{C}, \mathrm{~S}_{n}^{C}, \mathrm{~S}_{n}^{C}$ e $\mathrm{KD}_{4}^{C}{ }_{n}^{C}$ ) é completo em relação à classe $\mathcal{M}_{n}$ (resp., $\mathcal{M}_{n}^{r}, \mathcal{M}_{n}^{r t}, \mathcal{M}_{n}^{r s t}$ e $\mathcal{M}_{n}^{\text {elt }}$ ).

\section{Demonstração.}

Seja $\varphi$ válida em relação a $\mathcal{M}_{n}$ e suponha que $\varphi$ não é consistente, isto é, $\forall \neg \neg \varphi$ em $\mathrm{K}_{n}^{C}$ (resp., $\mathrm{T}_{n}^{C}, \mathrm{~S} 4_{n}^{C}, \mathrm{~S} 5_{n}^{C}$ e KD45 $5_{n}^{C}$ ), então pelo teorema anterior temos que $\neg \varphi$ é satisfatível em alguma estrutura de $\mathcal{M}_{n}$ (resp., $\mathcal{M}_{n}^{r}, \mathcal{M}_{n}^{r t}$, $\mathcal{M}_{n}^{r s t}$ e $\mathcal{M}_{n}^{\text {elt }}$ ) contradizendo a validade de $\varphi$. Portanto segue a tese.

A próxima seção trata de um aspecto do conhecimento entre agentes que não pode ser capturado a partir da noção de conhecimento mútuo ou conhecimento comum, a idéia de formalizar o conceito de "inferência a partir da combinação de conhecimentos" entre os agentes de um grupo, sem necessariamente a noção de conhecimento mútuo.

\subsection{Conhecimento Distribuído}

Na seção anterior tratamos da noção de conhecimento comum baseada na idéia de conhecimento mútuo, porém podemos pensar em situações onde o conhecimento mútuo não ocorre e na possibilidade de manipular o conhecimento que é distribuído pelo grupo. Caracterizamos o conhecimento distribuído do seguinte modo: considere a implicação $\varphi \rightarrow \psi \operatorname{com} \varphi$ composto de várias partes, isto é, como sendo a conjunção $\varphi_{1} \wedge \varphi_{2} \wedge \cdots \wedge \varphi_{m}$; se cada agente de um grupo $G$ conhece uma parte de $\varphi$, então os agentes em $G$, juntos, têm o conhecimento distribuído de $\psi$ quando se combinarem seus conhecimentos sobre $\varphi$. Formalizamos essa idéia com a inclusão dos axiomas $\left(1_{K_{n}^{D}}\right)$ e $\left(2_{K_{n}^{D}}\right)$ aos sistemas $\mathrm{K}_{n}, \mathrm{~T}_{n}, \mathrm{~S} 4_{n}$ e $\mathrm{S} 5_{n}$. como segue:

\section{Definição 4.6.}

Definimos $\mathrm{K}_{n}^{D}$ (resp., $\mathrm{T}_{n}^{D}, \mathrm{~S}_{n}^{D}$, e $\mathrm{S}_{n}^{D}$ ), como extensão de $\mathrm{K}_{n}$ (resp., $\mathrm{T}_{n}$, $\mathrm{S}_{n}$ e $\mathrm{S}_{n}$ ), com a inclusão dos axiomas:

$\left.1_{K_{n}^{D}}\right) D_{\{i\}} \varphi \leftrightarrow K_{i} \varphi, i \in \Delta$,

$\left.2_{\kappa_{n}^{D}}\right) D_{G} \varphi \rightarrow D_{G^{\prime}} \varphi$, se $G \subseteq G^{\prime}$.

Para verificar a validade dos axiomas necessitamos atribuir uma semântica para o operador $D_{G}$, esta deve ser tal que capture o fato de que a combinação de conhecimentos entre os agentes deve envolver unicamente o que é comum aos agentes envolvidos.

\section{Definição 4.7 .}


$\left.1_{D}\right)(\mathrm{M}, s) \models D_{G} \varphi$ se, e só se, $(\mathrm{M}, t) \models \varphi$ para todo $t$ tal que $(s, t) \in \bigcap_{i \in G} \mathcal{K}_{i}$.

\section{Proposição 4.9.}

Para qualquer fórmula $\varphi$ e $\mathrm{M}$ em $\mathcal{M}_{n}$ vale:

i) $\mathrm{M} \models D_{\{i\}} \varphi \leftrightarrow K_{i} \varphi, i \in \Delta$,

ii) $\mathrm{M} \models D_{G} \varphi \rightarrow D_{G^{\prime}} \varphi$, se $G \subseteq G^{\prime}$.

\section{Demonstração.}

A demonstração segue esquema análogo ao da Proposição 4.2 .

A semântica atribuída ao operador $D_{G}$ nos permite formalizar a discussão feita no início da seção sobre a inferência a partir de conhecimento distribuído. Afirmamos que: para quaisquer $\varphi_{1}, \ldots, \varphi_{m}, \psi \in \mathrm{K}_{n}^{D}(\Phi)$ e $\mathrm{M}$ de $\mathcal{M}_{n}$ se $\mathrm{M} \models\left(\varphi_{1} \wedge \cdots \wedge \varphi_{m}\right) \rightarrow \psi$, então $\mathrm{M} \models\left(K_{i_{1}} \varphi_{1} \wedge \cdots \wedge K_{i_{m}} \varphi_{m}\right) \rightarrow D_{G} \psi$, para $G=\left\{i_{1}, i_{2}, \ldots, i_{m}\right\} \subseteq \Delta$. De fato, se $\mathrm{M} \not \models\left(K_{i_{1}} \varphi_{1} \wedge \cdots \wedge K_{i_{m}} \varphi_{m}\right) \rightarrow D_{G} \psi$, então há $w$ em $\mathrm{M}$ tal que $(\mathrm{M}, w) \models K_{i_{1}} \varphi_{1} \wedge \cdots \wedge K_{i_{m}} \varphi_{m}$ e $(\mathrm{M}, w) \not \models D_{G} \psi$, isto é, existe $s$ em $\mathrm{M}$ tal que $(w, s) \in \bigcap_{i \in G} \mathcal{K}_{i}$ e $(\mathrm{M}, s) \not \models \psi$. Por outro lado, pela hipótese, ou $(\mathrm{M}, s) \not \models \varphi_{1} \wedge \cdots \wedge \varphi_{m}$ ou $(\mathrm{M}, s) \vDash \psi$. Como estamos supondo $(\mathrm{M}, w) \vDash K_{i_{1}} \varphi_{1} \wedge \cdots \wedge K_{i_{m}} \varphi_{m}$ e temos $(w, s) \in \mathcal{K}_{i_{j}}$, para todo $i_{j}$ de $G$, decorre que $(\mathrm{M}, s) \models \psi$. A contradição garante a veracidade da afirmação.

Podemos ter situações em que um grupo de agentes pode conhecer fatos contaditórios $\left(D_{G}(\varphi \wedge \neg \varphi)\right.$, para $\left.|G| \geq 2\right)$. Este comportamento não se adequa ao esquema de axioma $K_{i} \varphi \rightarrow \neg K_{i} \neg \varphi$, que implica em $\neg K_{i}(\varphi \wedge \neg \varphi)$, motivo pelo qual não incluímos $\mathrm{KD}_{4} 5_{n}$ em nossos estudos. Por outro lado o operador $D_{G}$ mantém as características de $K_{i}$ em relação a outros esquemas de axiomas.

\section{Observação 4.2 .}

- $\mathrm{M} \models D_{G}(\varphi \rightarrow \psi) \rightarrow\left(D_{G} \varphi \rightarrow D_{G} \psi\right)$.

$D e(\mathrm{M}, w) \vDash D_{G}(\varphi \rightarrow \psi)$ tem-se que $(\mathrm{M}, s) \vDash \varphi \rightarrow \psi$, para todo $s$ tal que $(w, s) \in \bigcap_{i \in G} \mathcal{K}_{i}$. Ou seja $(\mathrm{M}, s) \not \models \varphi$ ou $(\mathrm{M}, s) \models \psi$. Como $(\mathrm{M}, w) \vDash D_{G} \varphi$, temos que $(\mathrm{M}, s) \models \psi$, para todo $s \operatorname{com}(w, s) \in \bigcap_{i \in G} \mathcal{K}_{i}$. Portanto, $(\mathrm{M}, w) \models D_{G} \psi$ e segue a afirmação.

- $\mathrm{M} \models\left(D_{G} \varphi \wedge D_{G} \psi\right) \leftrightarrow D_{G}(\varphi \wedge \psi)$.

A demonstração segue esquema análogo ao item anterior. 
- $S e \mathrm{M} \in \mathcal{M}_{n}^{r t}$, então $\mathrm{M} \models D_{G} \varphi \rightarrow D_{G} D_{G} \varphi$.

$S e(\mathrm{M}, w) \models D_{G} \varphi$, então $(\mathrm{M}, s) \models \varphi$ para todo s tal que $(w, s) \in \bigcap_{i \in G} \mathcal{K}_{i}$.

Para qualquer $t$ em $\mathrm{M}$ tal que $(s, t) \in \bigcap_{i \in G} \mathcal{K}_{i}$, temos que $(w, t) \in \bigcap_{i \in G} \mathcal{K}_{i}$, pois $\cap \mathcal{K}_{i}$ é transitivo, já que para cada $i$ de $G$ a relação $\mathcal{K}_{i}$ o é. Logo, $(\mathrm{M}, s) \models D_{G} \varphi$, para todo s tal que $(w, s) \in \bigcap_{i \in G} \mathcal{K}_{i} e(\mathrm{M}, w) \models D_{G} D_{G} \varphi$. Portanto, $\mathrm{M} \models D_{G} \varphi \rightarrow D_{G} D_{G} \varphi$.

- Analogamente, verifica-se que:

$$
\begin{aligned}
& \text { se } \mathrm{M} \in \mathcal{M}_{n}^{r s t}, \text { então } \mathrm{M} \models \neg D_{G} \varphi \rightarrow D_{G} \neg D_{G} \varphi, \\
& \text { se } \mathrm{M} \in \mathcal{M}_{n}^{\text {elt }} \text {, então } \mathrm{M} \models D_{G} \varphi \rightarrow \neg D_{G} \neg \varphi .
\end{aligned}
$$

Como nos sistemas anteriores, os resultados que buscamos são os que caracterizam o sistema $\mathrm{K}_{n}^{D}$ (resp., $\mathrm{T}_{n}^{D}, \mathrm{~S}_{n}^{D}$ e $\mathrm{S} 5_{n}^{D}$ ) em relação a $\mathcal{M}_{n}$ (resp., $\mathcal{M}_{n}^{r}, \mathcal{M}_{n}^{r t}$ e $\mathcal{M}_{n}^{r s t}$ ) como sendo correto e completo. A correção segue o esquema de demonstrações anteriores.

\section{Teorema 4.10.}

$\mathrm{K}_{n}^{D}$ (resp., $\mathrm{T}_{n}^{D}, \mathrm{~S} 4_{n}^{D}$ e $\mathrm{S} 5_{n}^{D}$ ) é correto em relação a $\mathcal{M}_{n}$ (resp., $\mathcal{M}_{n}^{r}, \mathcal{M}_{n}^{r t}$ $\left.e \mathcal{M}_{n}^{r s t}\right)$.

\section{Demonstração.}

Como a Proposição 4.9 garante a validade dos axiomas com relação às respectivas classes de estruturas, então a demonstração da correção de $K_{n}^{D}$ (resp., $\mathrm{T}_{n}^{D}, \mathrm{~S} 4_{n}^{D}$ e $S 5_{n}^{C}$ ) segue de modo análogo a de teoremas anteriores.

Para o caso da completude, necessitamos de algumas complementações técnicas. Vale observar que podemos pensar em conceber um agente sábio $K_{D_{G}}$ que possua o conhecimento combinado de todos os agentes de um grupo, o que nos possibilita tratar a completude de $\mathrm{K}_{n+1}^{D}$ (resp., $\mathrm{T}_{n+1}^{D}$, $S 4_{n+1}^{D}$ e $S 5_{n+1}^{D}$ ) em relação à classe $\mathcal{M}_{n+1}$ (resp., $\mathcal{M}_{n+1}^{r}, \mathcal{M}_{n+1}^{r t}$ e $\mathcal{M}_{n+1}^{r s t}$ ). De fato, a completude se verifica nessas condições.

\section{Definição 4.8.}

Considere $\Gamma_{\mathrm{AX}}$ um subconjunto de $\mathrm{K}_{n}^{D}(\Phi)$ contendo todas as instâncias de algum conjunto de axiomas. Nessas condições, dizemos que:

1) um fórmula $\varphi$ em $\Gamma_{\mathrm{AX}}$ é dita ser $\Gamma_{\mathrm{AX}}$-consistente se $\forall \neg \varphi$ em $\mathrm{K}_{n}^{D}+\Gamma_{\mathrm{AX}}$, caso contrário $\varphi$ é dito ser $\Gamma_{\mathrm{AX}}$-inconsistente. Denotamos por $\mathrm{K}_{n}^{D}+\Gamma_{\mathrm{AX}}$ o sistema formado pelas fórmulas em $\mathrm{K}_{n}^{D}(\Phi)$ e fechado sob os axiomas e regras em $\mathrm{K}_{n}^{D}$ e $\Gamma_{\mathrm{Ax}}$. A noção de conjuntos $\Gamma_{\mathrm{Ax}}$-consistentes e maximais $\Gamma_{\mathrm{Ax}}$-consistentes é como feito anteriormente para a noção de consistência. 
2) Uma $\Gamma_{A x}$-estrutura de Kripke é uma estrutura $\mathrm{M}$ tal que $(\mathrm{M}, w) \models \varphi$ para todo $w$ de $\mathrm{M}$ e todo $\varphi$ de $\Gamma_{\mathrm{Ax}}$.

3) Dizemos que $\varphi$ é $\Gamma_{\mathrm{Ax}}$-satisfativel se existe uma $\Gamma_{\mathrm{Ax}}$-estrutura de Kripke $\mathrm{M}$ e um mundo $w$ em $\mathrm{M}$ tal que $(\mathrm{M}, w) \vDash \varphi$. Nesse caso, dizemos simplesmente que $\varphi$ é $\Gamma_{\mathrm{Ax}}$-satisfativel em $\mathrm{M}$. Evidentemente $\varphi$ é satisfativel em $\mathrm{M}$ se $\Gamma_{\mathrm{Ax}}=\emptyset$.

4) Dado $|\Delta|=n$, denotamos por $\ddot{M}=\left\langle W, \pi_{W}, \mathcal{K}_{1}, \ldots, \mathcal{K}_{n}, \mathcal{K}_{D_{G}}\right\rangle$ uma pseudo-estrutura de Kripke, com $D_{G}$ sendo considerado um outro agente. Se $|\Delta|=1$, então $\stackrel{*}{\mathrm{M}}=\left\langle W, \pi_{W}, \mathcal{K}_{1}, \mathcal{K}_{D_{G}}\right\rangle$ e $\mathcal{K}_{1}=\mathcal{K}_{D_{G}}$.

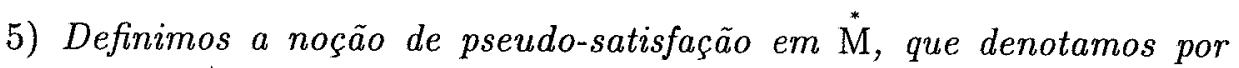
$(\stackrel{\mathrm{M}}{\mathrm{N}}, w) \stackrel{*}{\models} \varphi)$, de modo usual, exceto para fórmulas do tipo $D_{G} \psi$ : $(\stackrel{*}{\mathrm{M}}, w) \stackrel{*}{\models} D_{G} \psi$ se, e somente se, $\left(\stackrel{*}{\mathrm{M}}, w^{\prime}\right) \stackrel{*}{\models} \psi$, para todo $w^{\prime}$ em $\stackrel{*}{\mathrm{M}}$ tal que $\left(w, w^{\prime}\right) \in \mathcal{K}_{D_{G}}$.

6) Uma $\Gamma_{\mathrm{Ax}}$-pseudo-estrutura é uma pseudo-estrutura $\stackrel{*}{\mathrm{M}}$ tal que $(\stackrel{*}{\mathrm{M}}, w) \stackrel{*}{=} \varphi$ para todo $w$ de $\stackrel{*}{\mathrm{M}}$ e todo $\varphi$ de $\Gamma_{\mathrm{AX}}$.

7) Dizemos que uma fórmula $\varphi$ é $\Gamma_{\mathrm{Ax}}$-pseudo-satisfativel se existe uma $\Gamma_{\mathrm{Ax}}$-pseudo-estrutura de Kripke $\stackrel{*}{\mathrm{M}}$ e um mundo $w$ em $\stackrel{*}{\mathrm{M}}$ tal que $(\stackrel{\mathrm{M}}{\mathrm{M}}, w) \stackrel{*}{\models} \varphi$. Nesse caso, diz-se que $\varphi$ é $\Gamma_{\mathrm{Ax}}$-pseudo-satisfativel em $\stackrel{*}{\mathrm{M}}$.

Veja que a pseudo-satisfação é definida sobre pseudo-estruturas para tratar o operador $D_{G}$ como sendo um "agente sábio" que possui propriedades do conhecimento distribuido.

Esta situação permite-nos construir uma estrutura do tipo $\mathrm{M}_{\text {can }}$ que trate o operador $D_{G}$ exatamente como qualquer um dos $K_{i^{\prime} s}$, basta considerar, para cada conjunto $\Sigma \Gamma_{\mathrm{Ax}}$ consistente, o conjunto $\Sigma / D_{G}$ e a relação $\mathcal{K}_{D_{G}}$ tal que $\left(w_{\Sigma}, w_{\Sigma^{\prime}}\right) \in \mathcal{K}_{D_{G}}$ se, e somente se, $\Sigma / D_{G} \subseteq \Sigma^{\prime}\left(\Sigma / D_{G}\right.$ é definido de modo análogo à $\Sigma / K_{i}$, dada no Teorema 2.6). Do axioma $\left(1_{K_{n}^{D}}\right)$ segue que $\mathcal{K}_{\{i\}}$ (relação de acessibilidade para $D_{G} \operatorname{com} G=\{i\}$ ) é igual a $\mathcal{K}_{i}$. E podemos mostrar que: se $\varphi$ é $\Gamma_{\mathrm{AX}}$-consistente, então $\varphi$ é $\Gamma_{\mathrm{AX}}$-satisfativel, em particular, que $\varphi$ é satisfatível.

Proposição 4.11.

Se $\varphi$ é $\Gamma_{\mathrm{AX}}$-consistente, então $\varphi$ é $\Gamma_{\mathrm{AX}}$-pseudo-satisfativel.

Demonstração. [Fagin [22]]

A construção de $\stackrel{*}{\mathrm{M}}=\left\langle W, \pi_{W}, \mathcal{K}_{1}, \ldots, \mathcal{K}_{n}, \mathcal{K}_{D_{G}}\right\rangle, \operatorname{com}\left(w_{\Sigma}, w_{\Sigma^{\prime}}\right) \in \mathcal{K}_{D_{G}}$ 
se, e somente se, $\Sigma / D_{G} \subseteq \Sigma^{\prime}$, é idêntica à de $\mathrm{M}_{\text {can }}$, exceto pelo fato de utilizarmos a noção de $\Gamma_{\mathrm{Ax}}$ consistência ao invés da consistência usual. Nessas condições $\left(\stackrel{*}{\mathrm{M}}, w_{\Sigma}\right) \stackrel{*}{=} \psi$ se, e somente se, $\psi \in \Sigma$, para $\Sigma$ conjunto maximal $\Gamma_{\mathrm{Ax}}$-consistente. A demonstração é análoga a do Teorema 2.6 restando somente o caso em que $\psi$ é da forma $D_{G} \chi$ (a argumentação para os casos em que $\psi$ é uma proposição primitiva, tem forma de negação, implicação ou $K_{i} \chi$ é idêntica àquela feita no Teorema 2.6):

- se $\psi$ é da forma $D_{G} \chi$. Suponha $D_{G} \chi \in \Sigma$ e $\left(\stackrel{*}{\mathrm{M}}, w_{\Sigma}\right) \stackrel{*}{\not} D_{G} \chi$. Logo, $\chi \in \Sigma^{\prime}$, para todo $\Sigma^{\prime}$ maximal $\Gamma_{\mathrm{A} x}$ consistente que contenha $\Sigma / D_{G}$. Por outro lado, de $\left(\stackrel{*}{\mathrm{M}}, w_{\Sigma}\right) \stackrel{*}{\models} D_{G} \chi$ temos $\Sigma^{\prime \prime}$ maximal $\Gamma_{\mathrm{AX}}$ consistente tal que $\left(w_{\Sigma}, w_{\Sigma^{\prime \prime}}\right) \in \mathcal{K}_{D_{G}}$ e $\left(\stackrel{*}{M}, w_{\Sigma^{\prime \prime}}\right) \not{\forall} \chi$. Pela hipótese de indução $\chi \notin \Sigma^{\prime \prime}, \operatorname{logo} \neg \chi \in \Sigma^{\prime \prime}$ contradizendo o fato de $\chi \in \Sigma^{\prime \prime}$. Portanto, se $D_{G} \chi \in \Sigma$, então $\left(\stackrel{*}{\mathrm{M}}, w_{\Sigma}\right) \stackrel{*}{\models} D_{G} \chi$.

Agora, suponha que $\left(\stackrel{*}{M}, w_{\Sigma}\right) \stackrel{*}{\models} D_{G} \chi$ e $D_{G} \chi \notin \Sigma$. Então, $\chi \notin \Sigma^{\prime}$ para qualquer $\Sigma^{\prime}$ maximal $\Gamma_{\mathrm{Ax}}$ consistente que contenha $\Sigma / D_{G}$, logo $\neg \chi \in \Sigma^{\prime}$ para todo $\Sigma^{\prime}$ tal que $\left(w_{\Sigma}, w_{\Sigma^{\prime}}\right) \in \mathcal{K}_{D_{G}}$. Porém, de $\left(\stackrel{*}{\mathrm{M}}, w_{\Sigma}\right) \stackrel{*}{\models} D_{G} \chi$ tem-

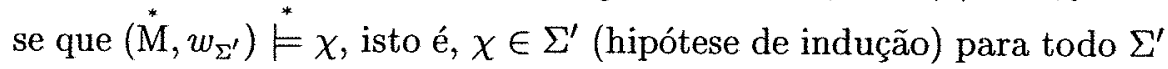
tal que $\left(w_{\Sigma}, w_{\Sigma^{\prime}}\right) \in \mathcal{K}_{D_{G}}$. Como $\Sigma^{\prime}$ é maximal $\Gamma_{A X}$ consistente temos uma contradição, portanto se $\left(\stackrel{*}{M}, w_{\Sigma}\right) \stackrel{*}{=} D_{G} \chi$, então $D_{G} \chi \in \Sigma$.

Continuando, como qualquer conjunto $\Sigma$ maximal $\Gamma_{A X}$ consistente contém $\Gamma_{\mathrm{Ax}}$, pois toda fórmula $\psi \mathrm{em} \Gamma_{\mathrm{Ax}}$ é instância de algum axioma, então temos $\vdash \psi$ e $\psi \in \Sigma$. Portanto, $\stackrel{M}{M}^{*} \Gamma_{\mathrm{Ax}}$ pseudo-estrutura e $\varphi$ é $\Gamma_{\mathrm{Ax}}$ pseudo-satisfatível.

Veja que, pelo item (4) na definição em 4.8, necessitamos verificar o caso em que $|\Delta|=1$; devemos mostrar que se $\stackrel{*}{M}=\left\langle W, \pi_{W}, \mathcal{K}_{1}, \mathcal{K}_{D_{G}}\right\rangle$, então $\mathcal{K}_{1}=\mathcal{K}_{D_{G}}$. Como $|\Delta|=1$, então $D_{G}=D_{\{1\}}$ e devemos ter a equivalência entre $K_{1}$ e $D_{\{1\}}$, isto é, $\Gamma_{\mathrm{AX}}$ contém o axioma $\left(1_{K_{n}^{D}}\right)$, logo segue a tese.

\section{Observação 4.3.}

$N a$ construção de $\stackrel{*}{M}=\left\langle W, \pi_{W}, \mathcal{K}_{1}, \ldots, \mathcal{K}_{n}, \mathcal{K}_{D_{G}}\right\rangle$, da proposição anterior, a relação $\mathcal{K}_{D_{G}}$ é subconjunto de $\bigcap_{i \in G} \mathcal{K}_{i}$, isto é, se $\left(w_{\Sigma}, w_{\Sigma^{\prime}}\right)$ é elemento de $\mathcal{K}_{D_{G}}$, então $\left(w_{\Sigma}, w_{\Sigma^{\prime}}\right) \in \mathcal{K}_{i}$, ou seja, $\Sigma / K_{i} \subseteq \Sigma^{\prime}$ para todo $i$ em $G$. De fato, seja $\psi$ fórmula em $\Sigma / K_{i}$, então $K_{i} \psi \in \Sigma$; como $\vdash K_{i} \psi \rightarrow D_{G} \psi$ (axiomas $\left(1_{\mathrm{K}_{n}^{D}}\right),\left(2_{\mathrm{K}_{n}^{D}}\right)$ e $\left.S H\right)$, segue que $D_{G} \psi \in \Sigma$, pois $\Sigma$ é maximal $\mathrm{r}_{\mathrm{Ax}}$-consistente. Desde que $\Sigma / D_{G} \subseteq \Sigma^{\prime}$, temos que $\psi \in \Sigma^{\prime}$.

A última proposição permite-nos afirmar que $\operatorname{se} \varphi$ é consistente, entâo $\varphi$ é satisfativel. 


\section{Proposição 4.12.}

Se $\varphi$ é $\Gamma_{\mathrm{AX}}$-consistente, então $\varphi$ é satisfativel numa estrutura em $\mathcal{M}_{n+1}$.

Demonstração. [Fagin [22]]

Pela proposição anterior, existe uma $\Gamma_{\mathrm{Ax}}$ pseudo-estrutura $\dot{M}^{*}$ tal que $\varphi$ é pseudo-satisfatível em $\stackrel{*}{\mathrm{M}}=\left\langle W, \pi_{W}, \mathcal{K}_{1}, \ldots, \mathcal{K}_{n}, \mathcal{K}_{D_{G}}\right\rangle$. Vamos considerar $\mathrm{M}=\left\langle W, \pi_{W}, \mathcal{K}_{1}, \ldots, \mathcal{K}_{n}, \mathcal{K}_{n+1}\right\rangle$ em $\mathcal{M}_{n+1} \operatorname{com} \mathcal{K}_{n+1}=\mathcal{K}_{D_{G}}$ e, para qualquer $\psi$, seja $\hat{\psi}$ a fórmula obtida pela substituição de todas as ocorrências de $D_{G}$ por $K_{n+1}$. Como $\varphi$ é pseudo-satisfatível em $\stackrel{*}{\mathrm{M}}$, segue da definição de pseudo-satisfação que $\hat{\varphi}$ é satisfatível em $\mathrm{M}$. Como $\mathcal{K}_{D_{G}}=\mathcal{K}_{n+1}$, então pela nota 4.3 segue que $\mathcal{K}_{n+1} \subseteq \bigcap_{i \in G} \mathcal{K}_{i}$, logo $(w, s) \in \mathcal{K}_{n+1}$ se, e só se, $(w, s) \in \mathcal{K}_{i}$, para todo $i$ em $G$. Logo, para todo $\psi$ e qualquer $w$ em $\mathrm{M}$, temos que $(\mathrm{M}, w) \vDash D_{G} \psi$ se, e só se, $(\mathrm{M}, w) \vDash K_{n+1} \psi$. Ou seja, para todo $\psi$ e qualquer $w$ em $\mathrm{M}$ temos que $(\mathrm{M}, w) \vDash \psi$ se, e só se, $(\mathrm{M}, w) \vDash \hat{\psi}$. Portanto, como $\hat{\varphi}$ é satisfatível em $M \varphi$, também o é.

E segue o teorema sobre a completude dos sistemas de conhecimento distribuído.

Teorema 4.13.

$\mathrm{K}_{n}^{D}$ (resp., $\mathrm{T}_{n}^{D}, \mathrm{~S}_{n}^{D}$ e $\left.\mathrm{S}_{n}^{D}\right)$ é completo em relação a $\mathcal{M}_{n+1}$ (resp., $\mathcal{M}_{n+1}^{r}$, $\mathcal{M}_{n+1}^{r t}$ e $\left.\mathcal{M}_{n+1}^{r s t}\right)$.

Demonstração. [Fagin, [22]]

Seja $\varphi$ válida em relação a $\mathcal{M}_{n}$ e suponha que $\varphi$ não é $\Gamma_{A X}$ consistente, isto é, $\forall \neg \neg \varphi$ em $\mathrm{K}_{n}^{D}+\Gamma_{\mathrm{AX}}$ (resp., $\mathrm{T}_{n}^{D}+\Gamma_{\mathrm{AX}}, \mathrm{S} 4_{n}^{D}+\Gamma_{\mathrm{AX}}$ e $S 5_{n}^{D}+\Gamma_{\mathrm{AX}}$ ). Então pela proposição anterior, temos que $\neg \varphi$ é satisfatível em alguma estrutura de $\mathcal{M}_{n+1}$ (resp., $\mathcal{M}_{n+1}^{r}, \mathcal{M}_{n+1}^{r t}$ e $\mathcal{M}_{n+1}^{r s t}$ ), contradizendo a validade de $\varphi$.

No entanto, o que desejamos é capturar todas as características de $\mathcal{K}_{D_{G}}$ pelo operador $D_{G}$, ou seja, obter uma estrutura em $\mathcal{M}_{n}$ tal que preserve a veracidade das fórmulas como na estrutura em $\mathcal{M}_{n+1}$ e de modo que $\mathcal{K}_{D_{G}}=\bigcap_{i \in G} \mathcal{K}_{i}$. Para alcançar nosso objetivo estabelecemos o seguinte plano, mostrar que:

- se $\varphi$ é $\Gamma_{\mathrm{A} X}$ consistente, então $\varphi$ é $\Gamma_{\mathrm{Ax}}$ pseudo-satisfatível.

- se $\varphi$ é $\Gamma_{A X}$ pseudo-satisfatível, então $\varphi$ é $\Gamma_{A X}$-satisfatível.

Logo, se tomarmos $\Gamma_{A x}=\emptyset$, temos a completude dos sistemas de conhecimento distribuído.

O primeiro item já foi tratado (Prop. 4.11); para o segundo devemos 
estabelecer a igualdade entre $\mathcal{K}_{D_{G}}$ e $\bigcap_{i \in G} \mathcal{K}_{i}$. Como já temos $\mathcal{K}_{D_{G}} \subseteq \bigcap_{i \in G} \mathcal{K}_{i}$, resta verificar em quais condições ocorre $\bigcap_{i \in G} \mathcal{K}_{i} \subseteq \mathcal{K}_{D_{G}}$. Grosso modo, construimos uma estrutura a partir de múltiplas cópias dos mundos que estão em $\bigcap \mathcal{K}_{i}$ e que não estão em $\mathcal{K}_{G}$, a fim de obtermos a igualdade entre $\mathcal{K}_{D_{G}}$ e $\bigcap_{i \in G}^{i \in G} \mathcal{K}_{i}$. Mais precisamente, dado $\mathrm{M}=\left\langle W, \pi_{W}, \mathcal{K}_{1}, \ldots, \mathcal{K}_{n}\right\rangle$, construímos $\mathrm{M}^{\prime}=\left\langle W^{\prime}, \pi^{\prime}{ }_{W^{\prime}}, \mathcal{K}^{\prime}{ }_{1}, \ldots, \mathcal{K}_{n}^{\prime}\right\rangle$ e uma função $f: W^{\prime} \rightarrow W$ tais que os mundos $w^{\prime}$ em $\mathrm{M}^{\prime}$ simulam os mundos $f\left(w^{\prime}\right)$ de $W$ em $M$ (simular significa que o conjunto de fórmulas $\varphi$ tal que $\left(\mathrm{M}^{\prime}, w^{\prime}\right) \models \varphi$ e $\left(\mathrm{M}, f\left(w^{\prime}\right)\right) \models \varphi$ é o mesmo). Para verificarmos as propriedades em $\mathrm{M}^{\prime}$ necessitamos desembaraçar a representação em grafo de $\mathrm{M}$ numa floresta (ou árvore). Desembaraçar $\mathrm{M}$ significa "remover os laços" em $M$ e a estatégia é como segue: dada uma estrutura $\mathrm{M}=\left\langle W, \pi_{W}, \mathcal{K}_{1}, \ldots, \mathcal{K}_{n}\right\rangle$, a construção dessa floresta (árvore) se dá por níveis. $\mathrm{O}$ primeiro nível $\mathrm{T}_{1}$ contém exatamente os mundos de $W$, então supondo definido $\mathrm{T}_{j}$ determinamos os outros níveis de modo indutivo: para cada $w$ de $W$, cada $v$ de $\mathrm{T}_{j}$ e cada $i$ de $\Delta$ definimos o mundo $z_{w, v, i}$. O conjunto $\mathrm{T}_{j+1}$ do nível $j+1$ consiste de todos os mundos $z_{w, v, i}$. Graficamente:

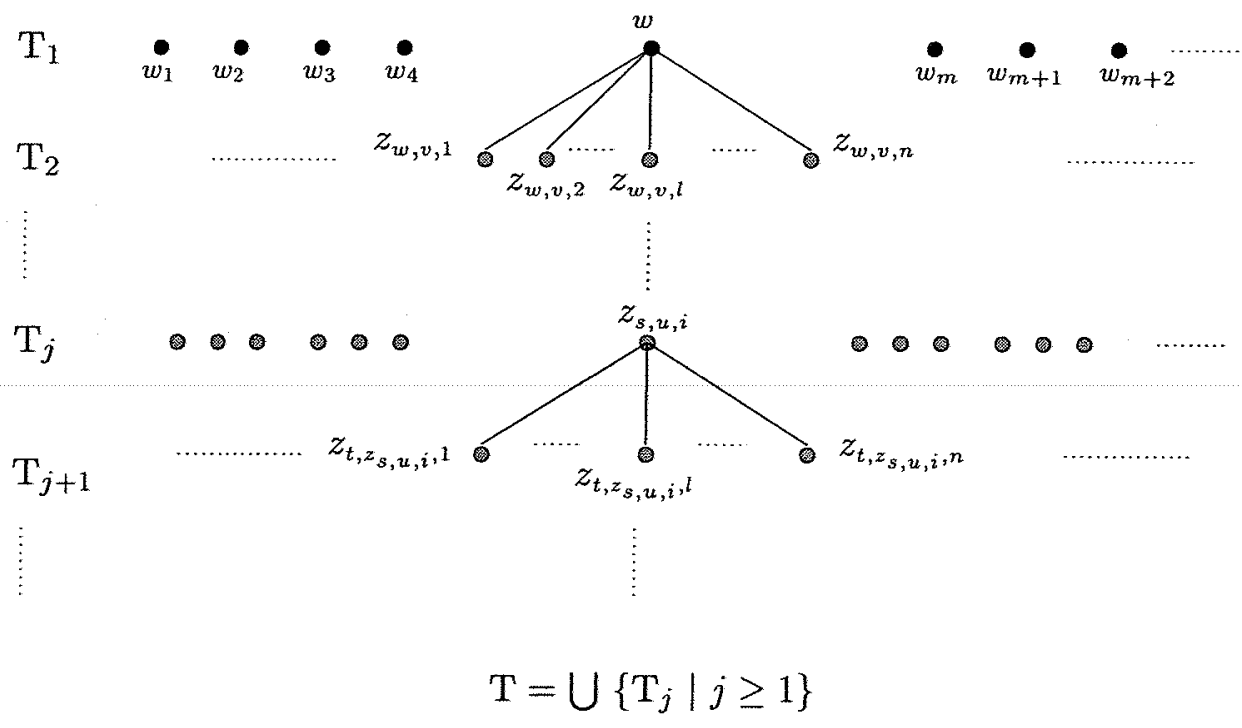

Figura 4.1: A floresta obtida a partir da estrutura M.

Referimo-nos ao mundo $z_{w, v, i}$ como sendo o $i$-filho de $v$ e a $v$ como sendo o pai de $z_{w, v, i}$. 


\section{Definição 4.9 .}

Seja $\mathrm{M}=\left\langle W, \pi_{W}, \mathcal{K}_{1}, \ldots, \mathcal{K}_{n}\right\rangle$ com $w$ e $s$ mundos em $\mathrm{M}$. Dado um caminho $\left\langle v_{1}, i_{1}, v_{2}, i_{2}, \ldots, v_{m-1}, i_{m-1}, v_{m}\right\rangle$ (Def. 4.3) de w a s uma redução desse caminho é obtido, substituindo cada subseqüência consecutiva maximal $\left\langle v_{q}, i_{q}, v_{q+1}, i_{q+1}, \ldots, v_{r-1}, i_{r-1}, v_{r}\right\rangle$, onde $i_{q}=i_{q+1}=\cdots=i_{r-1}$ por $\left\langle v_{q}, i_{q}, v_{r}\right\rangle$. Um caminho é dito ser reduzido se for idêntico à sua redução.

Dada uma estrutura $\mathrm{M}=\left\langle W, \pi_{W}, \mathcal{K}_{1}, \ldots, \mathcal{K}_{n}\right\rangle$, reconstruímos $\mathrm{M}^{\prime}$ utilizando a floresta (árvore) $\mathrm{T}$, vide figura acima, de modo a obtermos certos tipos de caminhos entre mundos.

Definição 4.10.

Dado $\mathrm{M}=\left\langle W, \pi_{W}, \mathcal{K}_{1}, \ldots, \mathcal{K}_{n}\right\rangle$, definimos $\mathrm{M}^{\prime}=\left\langle W^{\prime}, \pi^{\prime}{ }_{W^{\prime}}, \mathcal{K}^{\prime}{ }_{1}, \ldots, \mathcal{K}^{\prime}{ }_{n}\right\rangle$ como segue:

- Seja $\mathrm{T}=\bigcup\left\{\mathrm{T}_{j} \mid j \geq 1\right\}$ com $\mathrm{T}_{1}=W$ e, supondo definido $\mathrm{T}_{j}$, determinamos $\mathrm{T}_{j+1}$ como o conjunto de todos os mundos $z_{w, v, i}$ determinados para cada $w$ de $W$, cada $v$ de $\mathrm{T}_{j}$ e cada $i$ de $\Delta$ (vide figura 4.1).

- $W^{\prime}$ é o conjunto de mundos em $\mathrm{T}$, obtido a partir de $\mathrm{M}$.

- Definimos $f: W^{\prime} \rightarrow W$ tal que:

$$
\begin{cases}f(w)=w & \text { se } w \in \mathrm{T}_{1} \\ f\left(z_{w, v, i}\right)=w & \text { se } z_{w, v, i} \in \mathrm{T}_{j} \text { com } j \geq 2 .\end{cases}
$$

- As funções de valoração em $\pi_{W^{\prime}}{ }^{\prime}$ são tais que, para cada $w^{\prime}$ de $W^{\prime}$, temos $\pi_{w^{\prime}}^{\prime}=\pi_{f\left(w^{\prime}\right)}$

- Para cada i de $\Delta, \mathcal{K}_{i}^{\prime \prime}$ é o conjunto formado pelos pares $(w, s)$ tais que $(w, s) \in \mathcal{K}_{i}^{\prime \prime}$ se, e só se, $s$ é um $i$-filho de $w$ e $(f(w), f(s)) \in \mathcal{K}_{i}$.

- Consideramos as relações $\mathcal{K}_{i}^{\prime}$ conforme cada caso, para cada $i$ de $\Delta$ : - para $K_{n}^{D}$ é o fecho transitivo da relação $\mathcal{K}_{i}^{\prime \prime}$, - para $\mathrm{T}_{n}^{D}$ e $\mathrm{S}_{n}^{D}$, a relação $\mathcal{K}_{i}^{\prime}$ é o fecho reflexivo transitivo de $\mathcal{K}_{i}^{\prime \prime}$, - para $\mathrm{S}_{n}^{D}, \mathcal{K}_{i}^{\prime}$ é o fecho reflexivo, simétrico e transitivo de $\mathcal{K}_{i}^{\prime \prime}$.

Dados $w^{\prime}$ e s mundos em $\mathrm{M}^{\prime}$ a seqüência $\left\langle v_{1}^{\prime}, i_{1}, v_{2}^{\prime}, i_{2}, \ldots, v_{m-1}^{\prime}, i_{m-1}, v_{m}^{\prime}\right\rangle$ é um caminho primitivo de $w^{\prime}$ a $s^{\prime}$ se

- $v_{1}^{\prime}=w^{\prime}$

- $v_{m}^{\prime}=s^{\prime}$ 
- $v_{j}^{\prime} \in W^{\prime}$, para $1 \leq j<m$

- $i_{j} \in \Delta$, para $1 \leq j<m$

- ou $\left(v_{j}^{\prime}, v_{j+1}^{\prime}\right) \in \mathcal{K}_{i_{j}}^{\prime \prime}$ ou $\left(v_{j+1}^{\prime}, v_{j}^{\prime}\right) \in \mathcal{K}^{\prime \prime}{ }_{i_{j}}$, para $1 \leq j<m$.

Nesse caso, podemos chamar o caminho primitivo de $i_{j}$-caminho primitivo. Um caminho é não-redundante se não existir $j$ tal que $v_{j}=v_{j+2}$ e $i_{j}=i_{j+1}$.

Estas definições nos ajudam a tratar os laços devido à transitividade, simetria e reflexividade das relações $\mathcal{K}_{i}$. Note que $\left\langle v_{1}, i_{1}, \ldots, v_{m-1}, i_{m-1}, v_{m}\right\rangle$ é reduzido se $i_{j} \neq i_{j+1}$ e num caminho não-redundante não há subseqüência consecutiva da forma $\left\langle v_{j}, i_{j}, v_{j+1}, i_{j}, v_{j}\right\rangle$.

\section{Proposição 4.14.}

Dada uma estrutura $\mathrm{M}$ em $\mathcal{M}_{n}$, seja $\mathrm{M}^{\prime}$ a estrutura em $\mathcal{M}_{n}$ da definição 4.10. Então:

i) Se $w^{\prime}$ e s' estão em $\mathrm{M}^{\prime}$, então existe no máximo um caminho nãoredundante de $w^{\prime}$ para $s^{\prime}$.

ii) $\left(w^{\prime}, s^{\prime}\right) \in \mathcal{K}^{\prime}{ }_{i}$ se e só se existe um i-caminho primitivo de $w^{\prime}$ para $s^{\prime}$.

iii) se $\left(w^{\prime}, s^{\prime}\right) \in \mathcal{K}_{i}^{\prime}$, então $\left(f\left(w^{\prime}\right), f\left(s^{\prime}\right)\right) \in \mathcal{K}_{i}$.

\section{Demonstração.}

i) De fato, pois cada mundo possui no máximo um pai e os elementos em $\mathcal{K}_{i_{j}}^{\prime \prime}$ estão todos entre níveis adjacentes em $\mathrm{T}$.

ii) Para $K_{n}^{D}$ verifica-se a afirmação pela própria definição de $\mathcal{K}_{i}^{\prime}$. Para os outros casos suponha $\left(w^{\prime}, s^{\prime}\right) \in \mathcal{K}^{\prime}{ }_{i}$. Se $\left(w^{\prime}, s^{\prime}\right) \in \mathcal{K}^{\prime \prime}{ }_{i}$, então existe um $i$-caminho primitivo de $w^{\prime}$ para $s^{\prime}$. Por outro lado, se $\left(w^{\prime}, s^{\prime}\right) \notin \mathcal{K}^{\prime \prime}{ }_{i}$, então $\left(w^{\prime}, s^{\prime}\right)$ é incluso em $\mathcal{K}^{\prime}{ }_{i}$ por alguma das propriedades de fecho. Em particular, a transitividade de $\mathcal{K}^{\prime}{ }_{i}$ implica na existência de $t^{\prime}$ tal que $\left(w^{\prime}, t^{\prime}\right)$ e $\left(t^{\prime}, s^{\prime}\right)$ são elementos de $\mathcal{K}^{\prime \prime}{ }_{i}$, logo existe um $i$-caminho primitivo de $w^{\prime}$ para $s^{\prime}$.

A recíproca decorre de modo imediato, pois em todos os casos $\mathcal{K}_{i}^{\prime}$ é o fecho transitivo de $\mathcal{K}^{\prime \prime}{ }_{i}$, então se existe um $i$-caminho primitivo de $w^{\prime}$ para $s^{\prime}$, tem-se que $\left(w^{\prime}, s^{\prime}\right) \in \mathcal{K}^{\prime}{ }_{i}$.

iii) Para cada caso a propriedade se verifica: em $\mathrm{K}_{n}^{D}$ temos que $\left(f\left(w^{\prime}\right), f\left(s^{\prime}\right)\right) \in \mathcal{K}_{i}$ por construção. Nos outros casos utilizamos o item anterior e o fato de $\mathcal{K}_{i}^{\prime}$ ser o fecho transitivo de $\mathcal{K}_{i}^{\prime \prime}$. 
As relações $\mathcal{K}_{i}^{\prime}$, além das propriedades herdadas de $\mathcal{K}_{i}^{\prime \prime}$, determinam a qual classe de estruturas $\left(\mathcal{M}_{n}, \mathcal{M}_{n}^{r}, \mathcal{M}_{n}^{r t}\right.$ e $\mathcal{M}_{n}^{r s t}$, respectivamente) pertence $\mathrm{M}^{\prime}$.

\section{Definição 4.11.}

Dizemos que uma estrutura $\mathrm{M}$ é de tipo árvore (ou tree-like) se para quaisquer w e $s$ em $\mathrm{M}$ existe no máximo um caminho reduzido de w a s.

Observe que se $\mathrm{M}$ é de tipo árvore com $w$ e $s$ mundos distintos em $\mathrm{M}$ e tais que $(w, s) \in \mathcal{K}_{i}$ e $(w, s) \in \mathcal{K}_{j}$, então $i=j$.

\section{Definição 4.12.}

Dada uma estrutura $\mathrm{M}$ e um mundo $w$ nessa estrutura, denotamos por $\Xi_{(\mathrm{M}, w)}$ o conjunto de todas as fórmulas $\varphi$ tais que $(\mathrm{M}, w) \models \varphi$.

Outras propriedades de $\mathrm{M}^{\prime}$ são dadas pela próxima proposição.

\section{Proposição 4.15.}

Dada uma estrutura $\mathrm{M}$ em $\mathcal{M}_{n}$ temos que:

i) $\mathrm{M}^{\prime}$ é de tipo árvore.

ii) Para qualquer $w$ de $\mathrm{M}$, existe $w^{\prime}$ em $\mathrm{M}^{\prime}$, tal que $\Xi_{(\mathrm{M}, w)}=\Xi_{\left(\mathrm{M}^{\prime}, w^{\prime}\right)}$.

iii) Para qualquer $w^{\prime}$ de $\mathrm{M}^{\prime}$, existe $w$ em $\mathrm{M}$, tal que $\Xi_{(\mathrm{M}, w)}=\Xi_{\left(\mathrm{M}^{\prime}, w^{\prime}\right)}$.

Demonstração. [Fagin, [22]]

Dada $\mathrm{M}=\left\langle W, \pi_{W}, \mathcal{K}_{1}, \ldots, \mathcal{K}_{n}\right\rangle$, seja $\mathrm{M}^{\prime}=\left\langle W^{\prime}, \pi^{\prime}{ }_{W^{\prime}}, \mathcal{K}^{\prime}{ }_{1}, \ldots, \mathcal{K}^{\prime}{ }_{n}\right\rangle$, conforme a definição em 4.10 .

i) $\mathrm{M}^{\prime}$ é de tipo árvore.

De fato, tomemos $P$ e $P^{\prime}$, caminhos reduzidos de $w$ a $s$, vamos mostrar que $P=P^{\prime}$. Considere $P=\left\langle v_{1}, i_{1}, v_{2}, i_{2}, \ldots, v_{m-1}, i_{m-1}, v_{m}\right\rangle$ e $P^{\prime}=$ $\left\langle v_{1}^{\prime}, i_{1}^{\prime}, v_{2}^{\prime}, i_{2}^{\prime}, \ldots, v_{m-1}^{\prime}, i_{m-1}^{\prime}, v_{m}^{\prime}\right\rangle$. Como $\left(v_{j}, v_{j+1}\right) \in \mathcal{K}_{i_{j}}^{\prime}$, para $1 \leq$ $j \leq m$, segue que existe um $i_{j}$-caminho primitivo de $v_{j}$ para $v_{j+1} \mathrm{e}$, portanto, um $i_{j}$-caminho primitivo não-redundante de $v_{j}$ para $v_{j+1}$. Seja $\hat{P}$ o caminho primitivo de $w$ a $s$ obtido a partir de $P$, substituindo cada $i_{j}$-caminho primitivo não-redundante de $v_{j}$ para $v_{j+1}$ por $\left\langle v_{j}, i_{j}, v_{j+1}\right\rangle$, para $1 \leq j \leq m$. De modo análogo, podemos obter $\hat{P}^{\prime}$ a partir de $P^{\prime}$. Repare que $\hat{P}$ e $\hat{P}^{\prime}$ são caminhos primitivos nãoredundantes e, pela unicidade, temos que $\hat{P}=\hat{P}^{\prime}$. Como ambos são reduções de $P$ e $P^{\prime}$, respectivamente, ocorre que $P=P^{\prime}$. Portanto, $\mathrm{M}^{\prime}$ é de tipo árvore. 
ii) Para qualquer $w$ de $M$ existe $w^{\prime}$ em $M^{\prime}$ tal que $\Xi_{(M, w)}=\Xi_{\left(M^{\prime}, w^{\prime}\right)}$.

Para verificar que $\Xi_{\left(\mathrm{M}^{\prime}, w^{\prime}\right)}=\Xi_{\left(\mathrm{M}, f\left(w^{\prime}\right)\right)}$ basta mostrar que $\left(\mathrm{M}^{\prime}, w^{\prime}\right) \models \varphi$ se, e somente se, $\left(\mathrm{M}, f\left(w^{\prime}\right)\right) \models \varphi$. A demonstração é feita por indução na forma de $\varphi$. Se $\varphi$ é letra proposicional, segue da definição de que $\pi_{w^{\prime}}^{\prime}=\pi_{f\left(w^{\prime}\right)}^{\prime}$ e os casos em que $\varphi$ é negação ou implicação também decorrem de modo imediato. Agora, seja $\varphi$ da forma $K_{i} \psi$ : vamos mostrar que se $\left(\mathrm{M}, f\left(w^{\prime}\right)\right) \models \varphi$, então $\left(\mathrm{M}^{\prime}, w^{\prime}\right) \models \varphi$, demonstrando que se $\left(\mathrm{M}^{\prime}, w^{\prime}\right) \not \models \varphi$, então $\left(\mathrm{M}^{\prime}, w^{\prime}\right) \not \models \varphi$. Suponha que $\left(\mathrm{M}^{\prime}, w^{\prime}\right) \not \models K_{i} \psi$, logo existe $s^{\prime}$ em $\mathrm{M}^{\prime}$ tal que $\left(w^{\prime}, s^{\prime}\right) \in \mathcal{K}_{i}^{\prime}$ e $\left(\mathrm{M}^{\prime}, s^{\prime}\right) \not \neq \psi$. Da hipótese de indução e de $\left(f\left(w^{\prime}\right), f\left(s^{\prime}\right)\right) \in \mathcal{K}_{i}$, segue que $\left(\mathrm{M}^{\prime}, f\left(s^{\prime}\right)\right) \not \forall$. Logo, $\left(\mathrm{M}^{\prime}, f\left(w^{\prime}\right)\right) \not \models K_{i} \psi$.

Para a recíproca, suponha $\left(\mathrm{M}^{\prime}, f\left(w^{\prime}\right)\right) \not K_{i} \psi$, isto é, existe $s$ em $\mathrm{M}$ tal que $\left(f\left(w^{\prime}\right), s\right) \in \mathcal{K}_{i}$ e $(\mathrm{M}, s) \not \forall \psi$. Por construção de $\mathrm{T}, z_{s, w^{\prime}, i} \in \mathrm{T}$. Como $f\left(z_{s, w^{\prime}, i}\right)=s$ e $\left(f\left(w^{\prime}\right), s\right) \in \mathcal{K}_{i}$, temos que $\left(s, z_{s, w^{\prime}, i}\right) \in \mathcal{K}^{\prime \prime}{ }_{i}$. Logo, $\left(s, z_{s, w^{\prime}, i}\right) \in \mathcal{K}^{\prime}{ }_{i}$. Da hipótese de indução e $(\mathrm{M}, s) \not \psi \psi$ decorre que $\left(\mathrm{M}^{\prime}, z_{s, w^{\prime}, i}\right) \not=\psi$. Então, $\left(\mathrm{M}^{\prime}, s\right) \not \models K_{i} \psi$.

Portanto, para constatar que dado qualquer $w$ de M existe $w^{\prime}$ em $M^{\prime}$ tal que $\Xi_{(\mathrm{M}, w)}=\Xi_{\left(\mathrm{M}^{\prime}, w^{\prime}\right)}$, é só tomar $w^{\prime}=w$, pois $W=\mathrm{T}_{1} \subseteq \mathrm{T} \mathrm{e}$ $f(w)=w$, para $w \in W$.

iii) A demonstração é idêntica ao item anterior, considere $w=f\left(w^{\prime}\right)$

Com o que temos, podemos converter pseudo-satisfação em satisfação, isto é, vamos construir uma estrutura em $\mathcal{M}_{n}$ tal que $\mathcal{K}_{D_{G}}=\bigcap_{i \in G} \mathcal{K}_{i}$.

\section{Proposição 4.16.}

Se $\varphi$ é $\Gamma_{\mathrm{AX}}$-pseudo-satisfativel, então $\varphi$ é $\Gamma_{\mathrm{AX}}$-satisfativel.

Demonstração. [Fagin, [22]]

Vamos separar em dois casos: $|\Delta|=1$ e $|\Delta| \geq 2$. Para o primeiro caso, se $\varphi$ é $\Gamma_{A x}$ pseudo-satisfatível, então existe $\stackrel{M}{M}^{*}\left\langle W, \pi_{W}, \mathcal{K}_{1}, \mathcal{K}_{D_{G}}\right\rangle$ que satisfaz $\varphi$. Seja $\mathrm{M}=\left\langle W, \pi_{W}, \mathcal{K}_{1}\right\rangle$, estrutura em $\mathcal{M}_{1}$ (resp., $\mathcal{M}_{1}^{r}, \mathcal{M}_{1}^{r t}$ e $\mathcal{M}_{1}^{r s t}$ ). Como ${ }^{*}$ é uma pseudo-estrutura temos, em todos os casos, que $\mathcal{K}_{1}=\mathcal{K}_{D_{G}}$.

Veja que se $\psi$ é uma letra proposicional, ou tem forma de negação, implicação, $K_{i} \chi$ ou $D_{G} \chi$, então $(\stackrel{*}{M}, w) \stackrel{*}{=} D_{G} \psi$ se, e somente se, $(\mathrm{M}, w) \models K_{1} \psi$. Evidentemente, que $(\mathrm{M}, w) \vDash K_{1} \psi$ se e só se $(\mathrm{M}, w) \vDash D_{G} \psi$, para toda fórmula $\psi$, qualquer mundo $w$ e $G \subseteq \Delta$, não vazio.

Como $\varphi$ é pseudo-satisfatível em $\stackrel{*}{M}$, então $\varphi$ é satisfatível em M. De $\stackrel{*}{M}$ ser $\Gamma_{A x}$ pseudo-estrutura, segue que $M$ é $\Gamma_{A X}$ estrutura e $\varphi$ é $\Gamma_{A X}$ satisfatível. 
Vamos considerar o caso $|\Delta| \geq 2$. Pela proposição anterior (4.15) assumimos, sem perda de generalidade, que existem $\stackrel{*}{\mathrm{M}} \Gamma_{\mathrm{Ax}}$-pseudo-estrutura tree-like e um mundo $w$ tais que $(\stackrel{\mathrm{M}}{\mathrm{M}}, w) \stackrel{*}{\models} \varphi$. Observe que para $\mathrm{K}_{n}^{D}$ (resp., $\mathrm{T}_{n}^{D}, \mathrm{~S} 4_{n}^{D}$ e $\mathrm{S}_{n}^{D}$ ) a estrutura $\dot{\mathrm{M}}$ está em $\mathcal{M}_{n+1}$ (resp., $\mathcal{M}_{n+1}^{r}, \mathcal{M}_{n+1}^{r t}$ e $\left.\mathcal{M}_{n+1}^{r s t}\right)$. Então, considerando $\stackrel{*}{M}=\left\langle W, \pi_{W}, \dot{\mathcal{K}}_{1}, \ldots, \dot{\mathcal{K}}_{n}, \dot{\mathcal{K}}_{D_{G}}\right\rangle$ definimos $\mathrm{M}=\left\langle W, \pi_{W}, \mathcal{K}_{1}, \ldots, \mathcal{K}_{n}\right\rangle$. Para $\mathrm{K}_{n}^{D}$ e $\mathrm{T}_{n}^{D}$, a relação $\mathcal{K}_{i}$ como sendo $\mathcal{K}_{i}^{*} \cup \mathcal{K}_{D_{G}} ;$ note que para $T_{n}^{D}$, as relações $\mathcal{K}_{i}$ são reflexivas, pois $\dot{\mathcal{K}}_{i}$ e $\dot{\mathcal{K}}_{D_{G}}$ o são. Nos casos de $S 4_{n}^{D}$ e $S 5_{n}^{D}$, estamos considerando $\mathcal{K}_{i}$ como sendo o fecho transitivo de $\dot{\mathcal{K}}_{i} \cup \stackrel{*}{\mathcal{K}}_{D_{G}}$, logo, para $\mathrm{K}_{n}^{D}$ (resp., $\mathrm{T}_{n}^{D}, \mathrm{~S} 4_{n}^{D}$ e $\mathrm{S} 5_{n}^{D}$ ), temos que $\mathrm{M}$ é elemento de $\mathcal{M}_{n}$ (resp., $\mathcal{M}_{n+1}^{r}, \mathcal{M}_{n+1}^{r t}$ e $\mathcal{M}_{n+1}^{r s t}$ ). Vamos mostrar por indução na estrutura de $\psi$ que

$$
(\mathrm{M}, w) \models \psi \text { se, e somente se, }(\stackrel{*}{\mathrm{M}}, w) \stackrel{*}{\models} \psi
$$

Para o caso em que $\psi$ é uma letra proposicional é trivial, pois $\pi_{W}$ é o mesmo em $M^{*}$ e M. Se $\psi$ tem forma de negação ou implicação a demonstração é imediata. Os casos interessantes são para $\psi$ da forma $K_{i} \chi$ e $D_{G} \chi$.

- $\psi$ é da forma $K_{i} \chi$. Vamos mostrar que se $(\mathrm{M}, w) \models K_{i} \chi$, então $(\stackrel{*}{\mathrm{M}}, w) \stackrel{*}{\models} K_{i} \chi$. Suponha que $(\stackrel{*}{\mathrm{M}}, w) \stackrel{*}{\forall} K_{i} \chi$, então existe $s$ em $\stackrel{*}{\mathrm{M}}$ tal que $(w, s) \in \dot{\mathcal{K}}_{i}$ e $(\stackrel{\mathrm{M}}{,} s) \not{*} \chi$. Da hipótese de indução, $(\mathrm{M}, s) \not \neq \chi$. Logo, $(\mathrm{M}, w) \not K_{i} \chi$.

Agora, assuma que $(\stackrel{*}{\mathrm{M}}, w) \stackrel{*}{\models} K_{i} \chi$. Para mostrar que $(\mathrm{M}, w) \models K_{i} \chi$, basta verificarmos que $(\mathrm{M}, s) \models K_{i} \chi$, para todo $s$ tal que $(w, s) \in \mathcal{K}_{i}$. Para cada caso, pela definição de $\mathcal{K}_{i}$, se $(w, s) \in \mathcal{K}_{i}$, então existem $v_{1}, \ldots v_{m}$ em $W$ tais que:

$$
\begin{aligned}
& -v_{1}=w, \\
& -v_{m}=s \mathrm{e} \\
& \text { - ou }\left(v_{j}, v_{j+1}\right) \in \dot{\mathcal{K}}_{i}^{*} \text { ou }\left(v_{j}, v_{j+1}\right) \in \dot{\mathcal{K}}_{D_{G}}, \text { para } 1 \leq j<m .
\end{aligned}
$$

De fato, para $\mathrm{K}_{n}^{D}$ e $\mathrm{T}_{n}^{D}$, cada $\mathcal{K}_{i}$ foi definido como $\dot{\mathcal{K}}_{i} \cup \mathcal{K}_{D_{G}}$ e portanto existe um $i$-caminho primitivo de $w$ para $s$. Analogamente, para $S 4_{n}^{D}$ e $S 5_{n}^{D}$, pois cada $\mathcal{K}_{i}$ é o fecho transitivo de $\dot{\mathcal{K}}_{i} \cup \ddot{\mathcal{K}}_{D_{G}}$.

Resta mostrar que $\left(\stackrel{*}{M}, v_{j}\right) \stackrel{*}{\models} K_{i} \chi$ para $1 \leq j \leq m$. Fazemos isso por indução em $j$. Para $j=1$, nada a fazer. Suponha $\left(\stackrel{*}{\mathrm{M}}, v_{j}\right) \stackrel{*}{\models} K_{i} \chi$, para algum $j$ tal que $1 \leq j \leq l-1$, vamos mostrar que $\left(\stackrel{*}{\mathrm{M}}, v_{l}\right) \stackrel{F}{\models}^{*} K_{i} \chi$. Para o caso $\mathrm{K}_{n}^{D}$, de $\mathcal{K}_{i}=\stackrel{*}{\mathcal{K}}_{i} \cup \stackrel{*}{\mathcal{K}}_{D_{G}}$, e da hipótese, temos que $\left(\stackrel{*}{\mathrm{M}}, v_{l}\right) \stackrel{*}{\models} K_{i} \chi$. 
No caso $\mathrm{T}_{n}^{D}$ (resp., $\mathrm{S} 4_{n}^{D}$ e $\mathrm{S} 5_{n}^{D}$ ), usamos o esquema de axioma $T$. Do axioma $T$ e de $\left(\stackrel{*}{\mathrm{M}}, v_{j}\right) \stackrel{F}{F}^{*} K_{i} \chi$, segue que $\left(\stackrel{*}{\mathrm{M}}, v_{j}\right) \stackrel{*}{\models} K_{i} K_{i} \chi$. Como temos duas possibilidades: $\left(v_{j}, v_{j+1}\right) \in \dot{\mathcal{K}}_{i}$ ou $\left(v_{j}, v_{j+1}\right) \in \dot{\mathcal{K}}_{D_{G}}$, decorre que:

- se $\left(v_{j}, v_{j+1}\right) \in \dot{\mathcal{K}}_{i}$,

$\operatorname{de}\left(\stackrel{*}{\mathrm{M}}, v_{j}\right) \stackrel{*}{\models} K_{i} K_{i} \chi$, tem-se que $\left(\stackrel{*}{\mathrm{M}}, v_{j+1}\right) \stackrel{*}{\models} K_{i} \chi$.

- se $\left(v_{j}, v_{j}\right) \in{\stackrel{\mathcal{K}}{D_{G}}}_{\text {, }}$

pelo esquema de axioma $\left(1_{K_{n}^{D}}\right)$, temos que $K_{i} K_{i} \chi \rightarrow D_{\{i\}} K_{i} \chi$, $\operatorname{logo}\left(\stackrel{*}{\mathrm{M}}, v_{j}\right) \stackrel{*}{\models} K_{i} K_{i} \chi \rightarrow D_{\{i\}} K_{i} \chi$ e $\left(\stackrel{*}{\mathrm{M}}, v_{j}\right) \stackrel{*}{\models} D_{\{i\}} K_{i} \chi$. Da hipótese, decorre que $\left(\stackrel{*}{\mathrm{M}}, v_{j+1}\right) \stackrel{*}{\models} K_{i} \chi$.

Portanto, $\left(\stackrel{*}{\mathrm{M}}, v_{l}\right) \stackrel{*}{\models} K_{i} \chi$, para $1 \leq l \leq m$. E segue que $(\stackrel{*}{\mathrm{M}}, s) \stackrel{*}{\models} K_{i} \chi$, $\operatorname{logo}(\stackrel{*}{M}, s) \stackrel{*}{\models} \chi$. Pela hipótese de indução, $(\mathrm{M}, s) \models K_{i} \chi$.

- $\psi$ é da forma $D_{G} \chi$. Novamente, assuma que $(\stackrel{*}{\mathrm{M}}, w) \ddot{\forall} D_{G} \chi$, então existe $s$ em $\stackrel{*}{M}$ tal que $(w, s) \in \stackrel{*}{\mathcal{K}}_{D_{G}}$ e $(\stackrel{*}{M}, s) \not ै \not \chi$. Da hipótese de indução, $(M, s) \not \models \chi$. Como $\dot{\mathcal{K}}_{D_{G}} \subseteq \mathcal{K}_{i}$, para cada $i$ de $G$, segue que $(w, s) \in \mathcal{K}_{i}$, para cada $i$ de $G$, e $(\mathrm{M}, w) \not \neq D_{G} \chi$.

Para a recíproca: se $(\stackrel{*}{\mathrm{M}}, w) \stackrel{*}{\models} D_{G} \chi$, então $(\mathrm{M}, w) \vDash D_{G} \chi$, vamos supor $(\mathrm{M}, w) \not \models D_{G} \chi$ e mostrar que $(\stackrel{*}{\mathrm{M}}, w) \not \models * D_{G} \chi$. Pela suposição, existe $s$ em $W$ tal que $(w, s) \in \mathcal{K}_{i}$, para $i$ em $G$, e $(\mathrm{M}, s) \not \neq \chi$. Pela hipótese de indução, tem-se que $(\stackrel{*}{\mathrm{M}}, s) \not{\forall} \chi$. De $(w, s) \in \mathcal{K}_{i}$, há um caminho $P_{1}=\left\langle v_{1}, i_{1}, v_{2}, i_{2}, \ldots, v_{m-1}, i_{m-1}, v_{m}\right\rangle$ de $w$ a $s$ em $\stackrel{*}{M}$ tal que cada $i_{j}$ é 1 ou $D_{G}$. Podemos assumir que $P_{1}$ é um caminho reduzido. Analogamente, há um caminho $P_{2}=\left\langle u_{1}, i_{1}, v_{2}, i_{2}, \ldots, u_{m-1}, i_{m-1}, u_{m}\right\rangle$ de $w$ a $s$ em $\stackrel{*}{\mathrm{M}}$ tal que cada $i_{j}$ é 2 ou $D_{G}$ (por hipótese $n \geq 2$ ). Como M é de tipo árvore, há no máximo um caminho reduzido de $w$ a $s \mathrm{em}$ $\stackrel{*}{\mathrm{M}}, \operatorname{logo} P_{1}=P_{2}$. Ou seja, todo $i_{j}$ em $P_{1}$ é $D_{G}$. Do fato de $P_{1}$ ser um caminho reduzido, o comprimento de $P_{1}$ é 1 , isto é, $(w, s) \in{\stackrel{\mathcal{K}}{D_{G}}}$. Por fim, de $(\stackrel{*}{M}, s) \stackrel{*}{\forall} \chi$ decorre $(\stackrel{*}{M}, s) \stackrel{*}{\forall} D_{G} \chi$.

Portanto vale que se $\varphi$ é $\Gamma_{\mathrm{A} X}$ pseudo-satisfatível, então $\varphi$ é $\Gamma_{\mathrm{A} X}$-satisfatível.

Com os resultados das proposições 4.12 e 4.16 podemos afirmar que:

\section{Proposição 4.17.}

Se $\varphi$ é $\Gamma_{\mathrm{AX}}$-consistente, então $\varphi$ é $\Gamma_{\mathrm{AX}}$-satisfatível. 
Demonstração. [Fagin, [22]]

A demonstração é imediata de 4.12 e 4.16 .

Pela proposição anterior, segue que se $\varphi$ é $\Gamma_{A x}$ satisfatível em particular $\varphi$ é satisfativel e para $\Gamma_{\mathrm{AX}}=\emptyset$, segue a completude dos sistemas de conhecimento distribuído.

Teorema 4.18.

$\mathrm{K}_{n}^{D}$ (resp., $\mathrm{T}_{n}^{D}, \mathrm{~S}_{n}^{D}$ e $\mathrm{S} 5_{n}^{D}$ ) é completo em relação a $\mathcal{M}_{n}$ (resp., $\mathcal{M}_{n}^{r}$, $\mathcal{M}_{n}^{r t}$ e $\left.\mathcal{M}_{n}^{r s t}\right)$.

Demonstração. [Fagin, [22]]

A partir da Proposição 4.17, segue de modo análogo às demonstrações dos teoremas de completude anteriores.

Vimos no capítulo anterior que o conjunto de fórmulas válidas de $\mathcal{M}_{n}$ é caracterizado por $\mathrm{K}_{n}$ e que o conjunto de várias subclasses de $\mathcal{M}_{n}$ $\left(\mathcal{M}_{n}^{r}, \mathcal{M}_{n}^{r t}, \mathcal{M}_{n}^{r s t}\right.$ e $\left.\mathcal{M}_{n}^{\text {elt }}\right)$ são caracterizados por outros sistemas $\left(\mathrm{T}_{n}, \mathrm{~S}_{n}\right.$, $\mathrm{S} 5_{n}$ e KD45 ${ }_{n}$, respectivamente). Neste capítulo, verificamos que esta propriedade se mantém, mesmo com a inclusão dos operadores $C_{G}$ e $D_{G}$ ao sistema $K_{n}$ e suas extensões. Finalmente, ao término dos capítulos 2 e 3 observamos a equivalência entre $\vdash \mathrm{e} \models \mathrm{em}$ todos os sistemas de conhecimento apresentados. $\mathrm{O}$ que permite falar sobre consistência e provabilidade em termos de satisfatibilidade e validade, porém há uma diferença na forma em que os resultados foram apresentados, as estruturas $M_{\text {can }}$ e as baseadas em $\stackrel{*}{M}$ diferem das $M_{\varphi}$ quanto à construtibilidade efetiva, pois as últimas são finitas e possibilita tratar o problema de decidir se uma dada fórmula é provável e portanto válida em alguma classe de estruturas. O assunto do próximo capítulo é resolver a questão de decidibilidade e analisar a complexidade computacional do problema da satisfatibilidade nos diversos sistemas de conhecimento apresentados. 

Capítulo 5

\section{Decidibilidade e Complexidade}

Grosso modo, estamos interessados em classificar certos tipos de conjuntos de fórmulas, medindo a dificuldade em determinar se um objeto pertence ou não a um dado conjunto, se um tal método de decisão existir. $\mathrm{O}$ objetivo é estudar a complexidade do problema da validade para os sistemas de conhecimento, que é determinar se uma dada fórmula pertence ao conjunto de fórmulas válidas em relação a uma classe de estruturas.

Uma situação em que é interessante o reconhecimento de fórmulas válidas é o de um agente cuja base de informações é caracterizada por uma coleção $\Gamma$ de fórmulas. Suponha que $\varphi_{\Gamma}$ é a conjunção de todas as fórmulas em $\Gamma$; caso o agente queira saber se uma determinada informação $\psi$ decorre das informações que possui ele deve verificar a validade de $\varphi_{\Gamma} \rightarrow \psi$. Em situações práticas, podemos nos restringir a análise da validade de fórmulas da forma $K_{i} \varphi_{\mathrm{\Gamma}} \rightarrow K_{i} \psi$; para maiores detalhes consulte Halpern [24, sec., 3.2; $4.4 .1 \mathrm{e}$ 7.3] e Levesque [52].

Para tratar da complexidade computacional das lógicas ${ }^{1}$ de conhecimento, seguimos o seguinte roteiro: mostramos que existe um modo de decidir $^{2}$ a pertinência; a seguir estudamos a dificuldade do processo de decisão em termos de sua complexidade computacional, em função do tamanho da entrada. Para a segunda parte, necessitamos exibir os limitantes infe-

\footnotetext{
${ }^{1}$ Ao falarmos de uma lógica estamos nos referindo a um sistema axiomático e sua respectiva classe de estruturas. A complexidade computacional refere-se ao problema da satisfatibilidade para esses sistemas.

${ }^{2}$ Está fora do escopo deste texto fornecer um estudo aprofundado dos conceitos sobre decidibilidade.
} 
rior e superior dos recursos (tempo e espaço) gastos no processo de decisão. Exibir um limitante superior significa explicitar um algoritmo que resolva o problema. Por outro lado, para avaliar a eficiência de qualquer algoritmo que solucione o problema é necessário ter como parâmetro um limitante inferior, mostrar os recursos gastos por qualquer algoritmo que resolva o problema.

Seguindo o roteiro mencionado, dividimos este capítulo do seguinte modo: começamos estudando a decidibilidade dos sistemas de conhecimento, antems porém analisamos o problema de verificação de modelos (modelchecking). A seção seguinte introduz noções básicas sobre a teoria da complexidade e enuncia alguns resultados obtidos por Stockmeyer e Meyer em [76] e por Ladner em [48]. Na próxima seção examinamos alguns resultados sobre NP-completude para S5 e KD45. Na seção 5.4 exibimos os limitantes inferiores para $\mathrm{K}, \mathrm{T}$ e S4. Em 5.5 determinamos os limitantes superiores utilizando o método de tableaux. Finalizamos estendo o método para obtermos procedimentos de decisão para sistemas de conhecimento comum.

Ressaltamos que este capítulo visa complementar a caracterização dos sistemas de conhecimento dada pelos capítulos anteriores; uma outra forma de caracterizar os sistemas apresentados é de acordo com a classe de complexidade computacional a que pertencem. As demonstrações são todas baseadas em argumentações sobre propriedades das estruturas de Kripke, pois os resultados aqui expostos dependem fortemente da semântica adotada para os sistemas de conhecimento.

\subsection{Decidibilidade}

Somente a partir dos trabalhos de Church, Gödel e Turing é que se obteve uma definição robusta para a idéia de um problema ser computacionalmente decidível (ou solúvel), o que permitiu uma série de refinamentos, além da simples classificação entre decidível ou indecidível. Um exemplo desse refinamento foi dado por Cook (1971) mostrando que o problema da satisfatibilidade de CP é NP-completo. Diversos resultados se seguiram: Stockmeyer e Meyer (1973) mostraram que os problemas de decidibilidade podem ser precisamente caracterizados em termos de sua complexidade computacional, Ladner ([48] - 1977) demonstrou que o problema da satisfatibilidade para S5 é NP-completo e que a satisfatibilidade para K, T e S4 é PSPACEcompleto. Fischer e Ladner ([29] - 1979) obtiveram um limitante inferior para o PDL. Halpern e Moses ([36] - 1992) exibiram resultados para sistemas multi-agentes e mostraram que os sistemas envolvendo conhecimento comum tem complexidade EXPTIME, resultado baseado nos fatos obtidos da análise do PDL. 
Nesta seção resolvemos primeiramente o problema do model-checking, isto é, a questão de decidir se uma fórmula é satisfeita em um certo mundo numa dada estrutura de Kripke. A análise deste problema mostra-se essencial para o problema da validade, que é o tópico principal deste capítulo.

\section{Proposição 5.1.}

Dados $\mathrm{M}, w \in \mathrm{M}$ e $\varphi \in \mathrm{K}_{n}(\Phi)$, existe um algoritmo que verifica se $(\mathrm{M}, w) \vDash \varphi$ em tempo $O(\|\mathrm{M}\| \cdot|\varphi|)$.

Demonstração. [Halpern [36]]

Utilizamos a notação $O$ para denotar a medida do tempo computacional gasto: $O(\|\mathrm{M}\| \cdot|\varphi|)$ significa que existe uma constante $c>0$, independente da estrutura $M$ e de $\varphi$, tal que para quaisquer $M$ e $\varphi$ o tempo para verificar se $\varphi$ é satisfeita em M é feita em, no máximo, $c \cdot\|\mathrm{M}\| \cdot|\varphi|$ passos.

Para verificar a satisfatibilidade de $\varphi$ em M, basta checar a satisfatibilidade de todas as subfórmulas de $\varphi$ em cada mundo de M. A idéia é utilizar as subfórmulas de $\varphi$ para rotular cada mundo em $\mathrm{M}$; este procedimento pode ser feito em tempo $O(\|\mathrm{M}\| \cdot|\varphi|)$. De fato, considere a lista $\varphi_{1}, \ldots, \varphi_{m}$ contendo todos os elementos de $S u b(\varphi)$ em ordem não decrescente de tamanho, $\operatorname{logo} \varphi_{m}=\varphi$ e se $\varphi_{j}$ é subfórmula de $\varphi_{l}$, então $j<l$. Note que $m \leq|\varphi|$, pois $|S u b(\varphi)| \leq|\varphi|$ (Prop. 3.10). Se $\psi \in S u b(\varphi)$, então $\psi$ ocorre no rótulo de $s$ em M se $\pi_{s}(\psi)=\mathrm{T}$, caso contrário $\neg \psi$ ocorre no rótulo de $s$. Mostramos por indução sobre $j$ (índice da lista de subfórmulas) que a atribução de rótulos para cada mundo em $\mathrm{M}$ pode ser feito em tempo $O(\|\mathrm{M}\| \cdot j)$.

Para a base, $j=1, \varphi_{j}$ é letra proposicional e é imediato. Para o passo indutivo suponha que a afirmação se verifica para $j, 1 \leq j \leq l$, vamos mostrar que vale para $l+1$. Os casos em que $\varphi_{l+1}$ é letra proposicional, tem a forma de negação ou implicação são imediatos. Vamos verificar o caso em que $\varphi_{l+1}$ é da forma $K_{i} \varphi_{j} \operatorname{com} j<l+1$. Note que $K_{i} \varphi_{j}$ ocorre no rótulo de $s$ se, e somente se, todo $t$ tal que $(s, t) \in \mathcal{K}_{i}$ for rotulado com $\varphi_{j}$. Como pela hipótese de indução, cada mundo em $\mathrm{M}$ tem em seu rótulo $\varphi_{j}$ ou $\neg \varphi_{j}$, então para decidir se $\varphi_{l+1}$ ou $\neg \varphi_{l+1}$ ocorre num rótulo gastamos $O(\|\mathrm{M}\|)$ passos. Logo, a atribução de rótulos para cada mundo em $\mathrm{M}$ pode ser feita em tempo $O(\|\mathrm{M}\| \cdot j)$. Portanto, se considerarmos todas a subfórmulas de $\varphi$, gastamos $O(\|\mathrm{M}\| \cdot|\varphi|)$ passos. Finalmente, para verificar se $(\mathrm{M}, w) \vDash \varphi$ basta checar se $\varphi_{m}$ ocorre no rótulo de $w$.

Este resultado vale pode ser estendido para a linguagem $K_{n}^{C}(\Phi)$.

Proposição 5.2.

Dados $\mathrm{M}, w \in \mathrm{M}$ e $\varphi \in \mathrm{K}_{n}^{C}(\Phi)$ existe um algoritmo que verifica se $(\mathrm{M}, w) \models \varphi$ em tempo $O(\|\mathrm{M}\| \cdot|\varphi|)$. 


\section{Demonstração.}

Seja $G \subseteq \Delta \operatorname{com}|\Delta|=n$. Como na proposição anterior, procedemos por indução, restando analisar os casos em que $\varphi_{l+1}$ é da forma $E_{G} \varphi_{j}$ ou $C_{G} \varphi_{j}$ com $j<l+1$. Porém, ao invés de utilizarmos os elementos em $S u b(\varphi)$, usamos o conjunto $S u b_{C}(\varphi)$ (Def. 4.5) $\operatorname{com}\left|S u b_{C}(\varphi)\right| \leq(n+3) \cdot|\varphi|$ (Prop. 4.4). Considere $\varphi_{l+1}$ da forma $E_{G} \varphi_{j}$. Pela definição $\left(1_{C}\right)$ em 4.1 $\left((\mathrm{M}, w) \vDash E_{G} \psi\right.$ se, e só se, $(\mathrm{M}, w) \vDash K_{i} \psi$, para todo $\left.i \in G\right)$, de $|G| \leq n \mathrm{e}$ da hipótese de indução, cada mundo em $\mathrm{M}$ tem em seu rótulo $\varphi_{j}$ ou $\neg \varphi_{j}$, então para decidir se $\varphi_{l+1}$ ou $\neg \varphi_{l+1}$ ocorre num rótulo, gastamos $O(n \cdot\|\mathrm{M}\|)$ passos. Para o caso em que $\varphi_{l+1}$ é da forma $C_{G} \varphi_{j}$, o processo é análogo, basta considerar a Proposição 4.1, que diz que: $(\mathrm{M}, w) \models \mathrm{C}_{G} \psi$ se, e somente se, $(\mathrm{M}, s) \vDash \psi$ para todo $s$ G-atingivel a partir de $w$. Logo, a atribução de rótulos para cada mundo em $\mathrm{M}$ pode ser feito em $O((n \cdot j) \cdot\|\mathrm{M}\|)$ passos. Considerando todas as fórmulas em $S u b_{C}(\varphi)$ gastamos $O\left(n^{2} \cdot\|\mathrm{M}\| \cdot|\varphi|\right)$, isto é, $O(\|\mathrm{M}\| \cdot|\varphi|)$ passos. Analogamente, para verificar se $(\mathrm{M}, w) \vDash \varphi$, basta checar se $\varphi_{m}$ ocorre no rótulo de $w$.

Em geral, não há como verificarmos se uma fórmula é verdadeira em um dado mundo numa dada estrutura de Kripke se esta for infinita, porém pelas proposições anteriores a checagem é factível para estruturas finitas. Note que os resultados das proposições apresentadas independem do número de agentes e se verificam para qualquer subclasse de $\mathcal{M}_{n}$. Apesar de não termos apresentado estruturas finitas para os sistemas de conhecimento distribuído, estes são decidíveis ( $c f$. Fagin[24, pág. 64]), porém não fazem parte de nossos estudos.

Pelos teoremas 3.13 e 4.7 sabemos que se uma fórmula $\varphi$ de $K_{n}(\Phi)$ ou de $K_{n}^{C}(\Phi)$ é consistente, então ela é satisfatível em alguma estrutura finita. $\mathrm{O}$ que permite a analisar o problema da validade ${ }^{3}$ : dada uma fórmula $\varphi$ e uma classe de estruturas $\mathcal{M}_{n}$, exibir uma algoritmo que decide se $\varphi$ é válida em $\mathcal{M}_{n}$ (isto vale para qualquer subclasse de $\mathcal{M}_{n}$ ). Como o problema da validade e o da satisfatibilidade são complementares, pois uma fórmula é válida se, e somente se, sua negação não é satisfativel, temos que se existir um algoritmo que decide a satisfatibilidade de $\varphi$, podemos testar a validade de $\varphi$ tomando como entrada $\neg \varphi$ e vice-versa. Assim, pelas proposições anteriores afirmamos o seguinte resultado.

\section{Corolário 5.3.}

O problema da validade para $\mathcal{M}_{n}$ (resp., $\mathcal{M}_{n}^{r}, \mathcal{M}_{n}^{r t}, \mathcal{M}_{n}^{r s t}$ e $\left.\mathcal{M}_{n}^{e l t}\right)$ é decidivel.

\footnotetext{
${ }^{3}$ Lembramos que o problema da validade, para os sistemas aqui apresentados, torna-se interessante nos casos em que a classe das estruturas é a classe de todas as estruturas sobre alguma classe de frames.
} 


\section{Demonstração.}

Existe um algoritmo que decide se uma dada fórmula $\varphi$ é válida $\mathrm{em}$ relação a $\mathcal{M}_{n}$ (resp., $\mathcal{M}_{n}^{r}, \mathcal{M}_{n}^{r t}, \mathcal{M}_{n}^{r s t}$ e $\mathcal{M}_{n}^{\text {elt }}$ ). De fato, basta observar que $\varphi$ é satisfatível em relação a $\mathcal{M}_{n}$ (resp., $\mathcal{M}_{n}^{r}, \mathcal{M}_{n}^{r t}, \mathcal{M}_{n}^{r s t}$ e $\mathcal{M}_{n}^{\text {elt }}$ ) se, e somente se, $\neg \varphi$ não for satisfatível em relação a $\mathcal{M}_{n}$ (resp., $\mathcal{M}_{n}^{r}, \mathcal{M}_{n}^{r t}, \mathcal{M}_{n}^{r s t}$ e $\mathcal{M}_{n}^{\text {elt }}$ ). Logo, pelas proposições 5.1 e 5.2 , basta checar se $\neg \varphi$ é satisfativel em relação à $\mathcal{M}_{n}$ (resp., $\mathcal{M}_{n}^{r}, \mathcal{M}_{n}^{r t}, \mathcal{M}_{n}^{r s t}$ e $\mathcal{M}_{n}^{\text {elt }}$ ).

E como conseqüencia dos teoremas de correção e completude, que mostram a equivalência entre $\vdash \mathrm{e} \models$, temos que:

\section{Corolário 5.4.}

O problema da provabilidade para $\mathrm{K}_{n}\left(\right.$ resp. $, \mathrm{T}_{n}, \mathrm{~S}_{n}, \mathrm{~S}_{n}$ e $\left.\mathrm{KD}_{4} 5_{n}\right)$ e $\mathrm{K}_{n}^{C}$ (resp., $\mathrm{T}_{n}^{C}, \mathrm{~S} 4_{n}^{C}, \mathrm{~S} 5_{n}^{C}$ e $\mathrm{KD} 45_{n}^{C}$ ) é decidivel.

\section{Demonstração.}

Pelos teoremas de correção e completude, de cada um dos sistemas mencionados, decorre que $\varphi$ tem uma dedução em $\mathrm{K}_{n}$ (resp., $\mathrm{T}_{n}, \mathrm{~S} 4_{n}, \mathrm{~S} 5_{n}$ e $\mathrm{KD}_{4} 5_{n}$ ) e $\mathrm{K}_{n}^{C}$ (resp., $\mathrm{T}_{n}^{C}, \mathrm{~S} 4_{n}^{C}, \mathrm{~S} 5_{n}^{C}$ e KD45 $5_{n}^{C}$ ) se, e somente se, $\varphi$ é válida em relação a $\mathcal{M}_{n}$ (resp., $\mathcal{M}_{n}^{r}, \mathcal{M}_{n}^{r t}, \mathcal{M}_{n}^{r s t}$ e $\mathcal{M}_{n}^{\text {elt }}$ ).

Note que este último resultado apenas constata que a fórmula $\varphi$ possui uma dedução, não exibe a dedução no respectivo sistema axiomático. O leitor poderá encontrar em [24, págs. 65, 91 e 92] dicas para a construção efetiva de uma prova (dedução) para $\varphi$.

Uma vez que mostramos que os problemas mencionados são decidíveis, devemos analisar o quão complexo é este processo, ou seja, vamos estudar a complexidade computacional do problema de decisão. Isto pois os problemas decidíveis podem ser caracterizados de acordo com a classe de complexidade computacional a que pertencem ( $c f$. Stockmeyer [75]). A tabela a seguir antecipa os resultados que desejamos:

\begin{tabular}{||l|l|l||}
\hline NP-completo & PSPACE-completo & EXPTIME-completo \\
\hline \hline $\mathrm{S}_{1}, \mathrm{KD}_{4} 5_{1}$ & $\mathrm{~K}_{n}, \mathrm{~T}_{n}, \mathrm{~S} 4_{n} n \geq 1$ & $\mathrm{~K}_{n}^{C}, \mathrm{~T}_{n}^{C} n \geq 1$ \\
\hline & $\mathrm{S}_{n}, \mathrm{KD}_{4} n 5_{n} n \geq 2$ & $\mathrm{~S}_{n}^{C}, \mathrm{~S}_{n}^{C}, \mathrm{KD}_{4} 5_{n}^{C} n \geq 2$ \\
\hline
\end{tabular}

Tabela 5.1: Tabela da complexidade do problema da satisfatibilidade

Como acabamos de comentar, satisfatibilidade e validade estão intimamente relacionados e, portanto, os resultados apresentados na tabela podem ser interpretados em termos da complexidade do problema da validade para os sistemas de conhecimento. Por exemplo, pela tabela vemos que o problema da satisfatibilidade para $\mathrm{S}_{2}$ é $P S P A C E$-completo, mas PSPACE é fechado sob 
o complemento, então o problema da validade para $\mathrm{S}_{2}$ é PSPACE-completo.

Para esclarecer os termos utilizados na tabela e no parágrafo anterior elaboramos uma seção para expor a notação, os conceitos básicos e resultados necessários.

\subsection{Preliminares}

Apresentamos de modo resumido os conceitos necessários para tratarmos da complexidade computacional dos sistemas de conhecimento. Primeiramente, apresentamos a notação, alguns termos específicos e resultados básicos seguindo Hopcroft e Ullman [45, cap. 10]), por comodidade efetuamos uma ou outra alteração seguindo Garey e Johnson [30], Stockmeyer e Meyer [76] e Ladner [48]. A seguir, introduzimos as classes de complexidade que nos interessam. Finalizamos a seção apresentando definições, noções e resultados sobre fórmulas booleanas quantificadas, conforme Ladner [48] e Stockmeyer e Meyer [76].

\subsubsection{Máquinas de Turing e Complexidade Computacional}

Adotamos as máquinas de Turing (MT) como modelo para medir a tempo e espaço gastos em uma computação ${ }^{4}$. Lembrando que, a uma MT, estão associados alfabetos de entrada $\left(\Sigma_{\text {In }}\right)$ e saída $\left(\Sigma_{\text {Out }}\right)$, uma fita de trabalho, um conjunto finito de estados, um estado inicial e uma função de transição. Dada uma entrada $\alpha$ (elemento de uma linguagem ${ }^{5} \Sigma_{\text {In }}^{*}$ ), uma computação em de MT sobre $\varphi$ é uma seqüencia finita de configurações de MT. Uma máquina de Turing é dita ser determinística se em qualquer passo (configuração) numa computação o próximo passo é unicamente determinado. Por outro lado, uma MT é näo-deterministica se houver passos em que o próximo passo não é unicamente determinado, dentro de um conjunto finito de escolhas.

O tempo de uma computação é medido pelo tamanho da seqüência de configurações da computação em função do tamanho da entrada, mais precisamente, dizemos que MT computa em tempo $t: \mathbb{N} \rightarrow \mathbb{N}$, se para cada $m$, qualquer entrada $\alpha$ de tamanho $|\alpha|=m$, a computação de MT sobre $\alpha$ tem comprimento menor igual a $t(m)$. O espaço utilizado por uma computação

\footnotetext{
${ }^{4} \mathrm{Não}$ entramos em detalhes da teoria da computação ou sobre as máquinas de Turing, ao leitor que desejar maiores informaçōes indicamos Hopcroft e Ullman [45, cap. 10]

${ }^{5}$ Dado um alfabeto $\Sigma$ denotamos por $\Sigma^{*}$ o conjunto de todas as sequêencias finitas compostas por elementos de $\Sigma$. Chamamos de linguagem qualquer subconjunto de $\Sigma^{*}$.
} 
é medido pela fita de trabalho, isto é, MT computa em espaço $s: \mathbb{N} \rightarrow \mathbb{N}$ se para cada $m$ qualquer entrada $\alpha$ de tamanho $m$ a computação em MT de $\alpha$ utiliza no máximo $s(m)$ células distintas na fita de trabalho. Dizemos que uma computação é log-espaço se para cada entrada $\alpha$ de tamanho $|\alpha|$, a fita de trabalho da MT utiliza no máximo $\log |\alpha|$ células distintas.

Considere $\Gamma \subseteq \Sigma_{\text {In }}^{*}$, uma linguagem, $\Gamma$ é decidivel (ou computável) se, e somente se, existe uma MT tal que MT gera algum símbolo em $\Sigma_{0 \mathrm{ut}}^{*}$, para qualquer $\alpha \in \Gamma$. Também dizemos que $\Gamma$ é decidivel em tempo (espaço) nãodeterminístico $r$ se $\Gamma$ é computável por uma MT não-determinística e cujo tempo (espaço) de computação é $r$ para qualquer entrada em $\Sigma_{\mathrm{In}_{\mathrm{n}}}^{*}$. Definimos, de modo análogo, a noção de computável em tempo (espaço) determinístico. Por fim, dizemos que uma função $f: \Sigma_{\text {In }}^{*} \rightarrow \Sigma_{\text {Dut }}^{*}$ é computável em tempo (espaço) $r$ se possui uma MT determinística que computa qualquer entrada de $\Sigma_{\text {In }}^{*}$ em tempo (espaço) $r$. Além disso, quando MT pára a computação sobre a entrada $\alpha$, exibe como saída $f(\alpha)$.

Uma função é dita ser computacionalmente redutivel a outras se esta requer menores recursos para sua computação. Mais precisamente, dados $\Gamma_{\text {In }} \subseteq \Sigma_{\text {In }}^{*}$ e $\Gamma_{\text {out }} \subseteq \Sigma_{\text {out }}^{*}$, dizemos que $\Gamma_{\text {In }}$ é redutivel em tempo polinomial a $\Gamma_{\text {out }}\left(\Gamma_{\text {In }} \leq_{\text {pol }} \Gamma_{\text {Out }}\right)$ se, e somente se, há uma função $f: \Sigma_{\text {In }}^{*} \rightarrow \Sigma_{\text {Out }}^{*}$ computável em tempo polinomial e tal que para todo $\alpha \in \Sigma_{\text {In }}^{*}, \alpha \in \Gamma_{\text {In }}$ se, e só se, $f(\alpha) \in \Gamma_{\text {Dut }}$. De modo análogo definimos a noção de log-espaço redutível. E por último, $\Gamma_{\text {In }} \leq_{\log -1 \text { in }} \Gamma_{\text {Dut }}$ se, e somente se, existe $f$ computável em log-espaço e uma constante $c$ positiva e não nula tal que $|f(\alpha)| \leq c \cdot|\alpha|$ para todo $\alpha \in \Gamma_{\text {In }}$. Conforme Stockmeyer e Meyer [76], as relações $\leq_{\log } \mathrm{e} \leq_{\log -\operatorname{lin}}$ são transitivas e $\leq_{l o g}$ é reflexiva. A seguir, utilizamos as máquinas de Turing para determinar classes de complexidades e as reduções para estabelecer relações entre essas classes.

\subsubsection{Classes de Complexidade}

As classes de complexidade que nos interessam são $P, N P, P S P A C E$, EXPTIME e NEXPTIME. Dizemos que uma linguagem $\Gamma \subseteq \Sigma_{\text {In }}^{*}$ pertence a classe $P$ (resp., NP, PSPACE, EXPTIME e NEXPTIME) se, dado um elemento, existe uma MT determinística (resp., não determinística, determinística, determinística e não determinística) que decide se $\alpha \in \Sigma_{\text {In }}^{*}$ pertence a $\Gamma$ em tempo polinomial (resp., tempo polinomial, espaço polinomial, tempo exponencial ( $2^{d \cdot|\alpha|}$ com $d$ constante) e tempo exponencial) em função do tamanho da entrada $(|\alpha|)$. Algumas relações entre as classes apresentadas são conhecidas, por exemplo, sabe-se que: $P \subseteq N P \subseteq$ PSPACE $\subseteq$ EXPTIME $\subseteq$ NEXPTIME, $P \neq$ EXPTIME e que $N P \neq$ NEXPTIME, porém é problema em aberto se 
as outras inclusões são ou não próprias. Outro fato conhecido é que o não-determinísmo não acrescenta nenhum ganho em relação a computação $P S P A C E$, isto é, são equivalentes as computações em PSPACE determinística ou não-determinística (um resultado de Savitch [72] implica que PSPACE = NPSPACE).

Para qualquer classe de complexidade $\mathcal{C}$, podemos definir a classe $c o-\mathcal{C}$ formada pelos conjuntos cujo complemento é elemento de $\mathcal{C}$. Veja que se MT decide a pertinência sobre um conjunto de modo determinístico, então é fácil converter MT numa máquina $\mathrm{MT}^{\prime}$ que decide a pertinência sobre o complemento do mesmo conjunto utilizando os mesmos recursos de tempo e espaço (MT' aceita a entrada se e somente se MT a rejeita). Logo, para qualquer classe determinística $\mathcal{C}$ vale que $\mathcal{C}=c o-\mathcal{C}$, em particular para $P$, PSPACE e EXPTIME. O mesmo não ocorre com algoritmos não-determinísticos, assim não sabemos se $N P=C O-N P^{6}$.

Caracterizamos as classes $\mathcal{C}$-dificil, $\mathcal{C}$-completa e $\mathcal{C}$-log-espaço-completo como segue: uma linguagem é dita estar na classe $\mathcal{C}$-dificil se para qualquer elemento de $\mathcal{C}$ houver uma redução em tempo polinomial à linguagem dada. Um elemento está na classe $\mathcal{C}$-completa se é elemento de $\mathcal{C}$ e $\mathcal{C}$-difícil. Dada uma classe $\mathcal{C}$, uma linguagem está em $\mathcal{C}$-log-espaço-completo se for elemento de $\mathcal{C}$ e se qualquer outro conjunto da classe é log-espaço-redutivel a ela. Vale lembrar que Cook ([14] - 1971) mostrou a existência de conjuntos log-espaço-completos na classe $N P$, enquanto que Stockmeyer e Meyer ([76] 1973) mostraram a existência de conjuntos log-espaço-completos em PSPACE (vide na próxima seção, fato 5.5 ).

Podemos exemplificar a utilização dessas classes através de um fato demonstrado por Cook. Ele mostrou que o problema de determinar se uma fórmula do cálculo proposicional clássico é satisfatível é NP-completo. Isto significa que se for possível encontrar um algoritmo que decida a satisfatibilidade de fórmulas em CP em tempo polinomial, então qualquer outro problema da classe $N P$ também possui um algoritmo que decide o problema em tempo polinomial. Como a satisfatibilidade e a validade são problemas complementares, se o problema da satisfatibilidade para alguma lógica é completa para alguma classe de complexidade $\mathcal{C}$, então o problema da validade é $c o-\mathcal{C}$, em particular o problema da validade de CP é co-NP-completo. Outras situações estão expostas na tabela 5.1. Como comentamos, o problema da satisfatibilidade para $\mathrm{S}_{2}$ é PSPACE-completo, isto é, existe um algoritmo que decide se uma fórmula é satisfativel em relação a $\mathcal{M}_{2}^{r s t}$ e cuja computação ocupa espaço polinomial, além disso qualquer problema PSPACE pode ser eficientemente reduzido ao problema da satisfatibilidade para $\mathrm{S}_{2}$.

\footnotetext{
${ }^{6}$ Veja que se $P=N P$, então $N P=c o-N P$, porém conjectura-se que $N P \neq c o-N P$.
} 
Por outro lado, como PSPACE é uma classe de complexidade determinística, então o problema da validade para $\mathrm{S}_{2}$ é também PSPACE-completo.

Diversas lógicas foram estudadas em termos da complexidade dos problemas de satisfatibilidade e validade, muitas sendo completamente caracterizadas pelas classes a que pertencem esses problemas. Para nós, são relevantes alguns resultados sobre a lógica das fórmulas booleanas quantificadas, pois nossos estudos são calcados nas análises feitas por Ladner [48], por exemplo, para mostrar que a satisfatibilidade para $\mathrm{K}, \mathrm{T}, \mathrm{S} 4, \mathrm{~S}_{2}$ e $\mathrm{KD} 45_{2}$ é PSPACE-difícil ele fez uso da lógica booleana quantificada. Apresentamos a seguir somente as idéias necessárias aos objetivos deste capítulo, não é nosso objetivo nos alongarmos sobre o assunto.

\subsubsection{Fórmulas Booleanas Quantificadas}

Basicamente, definimos a lógica das fórmulas booleanas quantificadas como sendo composta pela linguagem $\mathrm{CP}(\Phi)$. O conjunto $\mathrm{QBF}(\Phi)$ das fórmulas booleanas quantificadas é o menor conjunto contendo todas as fórmulas de $\mathrm{CP}(\Phi)$ e tal que se $\beta \in \mathrm{QBF}(\Phi)$ e $x \in \Phi$, então $\forall x \beta$ e $\exists x \beta$ também são elementos de $\mathrm{QBF}(\Phi)$. Os quantificadores $\forall$ e $\exists$ atuam sobre os valores verdade $T$ (ou 1) e $\perp$ (ou 0 ). Dizemos que uma fórmula booleana quantificada (ou $q b f$ ) é bem-formada se todas as variáveis estão quantificadas. Estamos interessados apenas nas $q b f$ 's bem-formadas e que estejam no formato prenex, isto é, fórmulas na forma: $Q_{1} x_{1} Q_{2} x_{2} \cdots Q_{m} x_{m} \beta\left(x_{1}, x_{2}, \ldots, x_{m}\right)$, com $Q_{j} \in\{\forall, \exists\}, x_{j}$ variável $(1 \leq j \leq m)$ e $\beta\left(x_{1}, x_{2}, \ldots, x_{m}\right)$ fórmula de $\mathrm{CP}(\Phi)$. Chamamos a fórmula $\beta$ de matriz da $q b f$. Dada uma $q b f$, dizemos que ela é válida se, e somente se, o valor-verdade de sua matriz for $1(T)$. Note que na lógica QBF não há distinção entre verdade e validade.

Intuitivamente, a leitura que fazemos da fórmula $\exists x \beta(x)$ é a de que existe uma valoração para $x$ tal que $\beta(x)$ é verdadeira, analogamente $\forall x \beta(x)$ diz que para qualquer valoração para $x \beta(x)$ é verdadeira. De modo mais geral, para: $Q_{1} x_{1} Q_{2} x_{2} \cdots Q_{m} x_{m} \beta\left(x_{1}, x_{2}, \ldots, x_{m}\right)$, se $Q_{j}=\exists$, então existe uma valoração para $x_{j}$ tal que $Q_{j+1} x_{j+1} \cdots Q_{m} x_{m} \beta\left(\cdots, x_{j+1}, \ldots, x_{m}\right)$ é verdadeira. Por outro lado, se $Q_{j}=\forall$, então para qualquer valoração para $x_{j}$ tem-se que $Q_{j+1} x_{j+1} \cdots Q_{m} x_{m} \beta\left(\cdots, x_{j+1}, \ldots, x_{m}\right)$ é verdadeira.

Podemos associar o processo de determinar se uma $q b f$ é verdadeira ou falsa à construção de uma árvore de valoração. Grosso modo, cada fórmula da forma $\forall x \beta(x)$ é substituida por $\beta_{0} \wedge \beta_{1}$ e $\exists x \beta(x)$ por $\beta_{0} \vee \beta_{1}$, tal que $\beta_{0}$ (resp., $\left.\left.\beta_{1}\right)\right)$ é $\beta(x)$ com todas as ocorrências de $x$ substituidas por 0 (resp., 1). Vejamos um exemplo: considere a fórmula $\forall p \exists q((p \rightarrow \neg q) \wedge(\neg q \rightarrow p))$ e a 
respectiva árvore das possíveis valorações das letras que ocorrem na matriz.

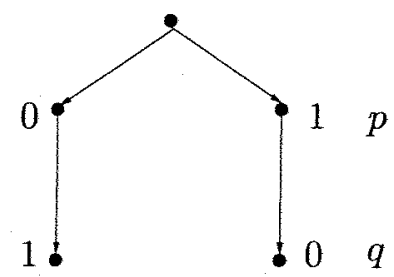

Figura 5.1: Árvore quantificada.

Efetivamente, a construção da árvore (atribuição de valores-verdade) se dá pela leitura da $q b f$, da esquerda para a direita; os nós da árvore são rotulados com as respectivas valorações das letras do escopo do quantificador. A valoração ocorre do seguinte modo: se o quantificador encontrado for o universal, todas as valorações (distintas) possíveis a variável de seu escopo são consideradas, isto é, tomamos os valores 1 e 0 . Por outro lado, se o quanficador for o existencial tomamos ou o valor 1 ou o valor 0 . Os conectivos lógicos funcionam como em CP. Assim, o quantificador universal ramificase em dois, enquanto que o quantificador existencial estende-se num único ramo. Veja que a validade de uma $q b f$ ocorre se, e somente se, existe uma árvore para a $q b f$ tal que as valorações asseguram que a matriz assume valor 1 em algum nó folha.

A correspondência entre as $q b f$ 's e as fórmulas modais dos sistemas de conhecimento é apresentada na seção 5.4 (Teor. 5.10). Por hora definimos certos conjuntos de $q b f$ 's utilizados por Stockmeyer e Meyer [76] e Ladner [48] para estabelecer alguns resultados sobre a complexidade para QBF.

Definimos os conjuntos $B_{j}$, para $j \in \mathbb{N}$ e $j \geq 1$, de modo indutivo (para maiores detalhes consultar [76]): $B_{1}=\{\beta(X) \mid \exists X[\beta(X) \leftrightarrow 1]\}$ $\operatorname{com} X \equiv x_{1} x_{2} \cdots x_{m}$ seqüência de variáveis que ocorrem em $\beta$. Se $j \geq 1$, $B_{j}=\left\{\beta\left(X_{1}, X_{2}, \ldots, X_{j}\right) \mid \exists X_{1} \forall X_{2} \exists X_{3} \cdots Q_{j} X_{j}\left[\beta\left(X_{1}, X_{2}, \ldots, X_{j}\right) \leftrightarrow 1\right]\right\}$ com $Q_{l}=\forall$ se $l$ par e $Q_{l}=\exists$ caso contrário. Por fim, $B_{\omega}=\bigcup_{j \in \mathbb{N}^{*}} B_{j}$. Veja que $B_{1}$ é o conjunto de todas as fórmulas satisfatíveis de CP e $B_{\omega}$ o conjunto de todas as $q b f$ 's válidas. E segue que:

\section{Fato 5.5.}

1) (Stockmeyer e Meyer [76]) B B é log-espaço-completo em PSPACE.

2) (Cook [14]) $B_{1}$ é log-espaço completo em NP.

O fato em (1) decorre da transitividade da relação $\leq_{\log }$ (cf. Stockmeyer 
[76]), diz que todo problema computável em PSPACE é log-espaço-redutível a uma linguagem, por exemplo, $\Gamma$ se pudermos mostrar que $B_{\omega}$ é log-espaçoredutível à $\Gamma$. Esta caracterização de $B_{\omega}$ e $B_{1}$ pela classe de complexidade à qual pertencem é utilizado direta ou indiretamente nas seções seguintes. Ladner, utilizando a transitividade de $\leq_{l o g}$ e o fato em (1), estabeleceu relações de complexidade, via reduções log-espaço, de $B_{\omega}$ com os sistemas modais entre $\mathrm{K}$ e S4 (cf. teor. 3.1 [48]). Essas relações são fundamentais para os resultados apresentados na seção 5.4. Ladner utilizou ${ }^{7}$ o fato em (2) para o estudo da complexidade de 55 , assunto da próxima seção.

\subsection{A classe de complexidade de S5 e KD45}

A partir desta seção iniciamos, propriamente dita, a análise das complexidades dos sistemas de conhecimento. Esta seção trata especificamente dos sistemas S5 e KD45. Desde que S5 e KD45 são extensões de CP e este é $N P$-completo, então supõe-se que S5 e KD45 estão em classes tão difíceis quanto CP. No entanto, Ladner mostrou que a adição de operadores modais, pelo menos para S5, não aumenta a complexidade, resultado análogo vale para KD45. A estratégia para mostrar que S5 e KD45 são NP-completos é verificar que a satisfatibilidade nesses sistemas ocorrem em estruturas finitas com poucos mundos possíveis.

\section{Proposição 5.6 (Ladner).}

Uma fórmula $\varphi$ é satisfativel em $\mathrm{S} 5$ se, e só se, é satisfeita numa estrutura $\mathcal{M}_{1}^{\text {rst }}$ com pelo menos $|\varphi|$ mundos.

\section{Demonstração. [Halpern [36]]}

Vamos mostrar que se $\varphi$ é satisfatível em S5, então ela é satisfeita numa estrutura em $\mathcal{M}_{1}^{r s t}$ com pelo menos $|\varphi|$ mundos; a recíproca é imediata. Suponha que existe $\mathrm{M} \in \mathcal{M}_{1}^{r s t}$ tal que $(\mathrm{M}, w) \models \varphi$, então, pela Proposição 3.7 , podemos afirmar, sem perda de generalidade, que $\mathrm{M}=\left\langle W, \pi_{W}, \mathcal{K}_{1}\right\rangle$ com $\mathcal{K}_{1}$ universal. Vamos construir uma estrutura $\mathrm{M}^{\prime}$ usando como referência um subconjunto de $\operatorname{Sub}(\varphi)$ tal que $\mathrm{M}^{\prime}=\left\langle W^{\prime}, \pi_{W}^{\prime}, \mathcal{K}^{\prime}\right\rangle \operatorname{com} W^{\prime} \subseteq W,\left|W^{\prime}\right| \leq|\varphi|$ e para todo $w^{\prime} \in W^{\prime}$ vale que $\left(\mathrm{M}^{\prime}, w^{\prime}\right) \models \psi$ se, e só se, $\left(\mathrm{M}, w^{\prime}\right) \models \psi$, para $\psi \in \operatorname{Sub}(\varphi)$.

Considere $\Gamma \subseteq S u b(\varphi)$, com $\Gamma$ contendo todas a fórmulas da forma $K_{1} \chi$ que sejam falsas em $w: \Gamma=\left\{\psi \in \operatorname{Sub}(\varphi) \mid \psi=K_{1} \chi e(\mathrm{M}, w) \vDash \neg K_{1} \chi\right\}$. Logo, para cada $K_{1} \chi$ de $\Gamma$, existe $s_{\chi} \in W$ tal que $\left(\mathrm{M}, s_{\chi}\right) \models \neg \chi$. Então,

\footnotetext{
${ }^{7} \mathrm{O}$ problema da satisfatibilidade em S5 é log-espaço-redutivel a $N P$ (cf. Ladner [48, teor. 6.2])
} 
definimos a estrutura $\mathrm{M}^{\prime}=\left\langle W^{\prime}, \pi_{W}^{\prime}, \mathcal{K}^{\prime}\right\rangle$ tal que $W^{\prime}=\{w\} \cup\left\{s_{\chi} \mid K_{1} \chi \in \Gamma\right\}$, $\pi_{W}^{\prime}$ a restrição de $\pi_{W}$ a $W^{\prime}$ e $\mathcal{K}^{\prime}$ universal em $W^{\prime}$. Como $|\Gamma| \leq S u b(\varphi) \leq|\varphi|$, então $\left|W^{\prime}\right| \leq|\varphi|$. Resta mostrar que $\left(\mathrm{M}, s^{\prime}\right) \models \psi$ se, e só se, $\left(\mathrm{M}^{\prime}, s^{\prime}\right) \models \psi$, para quaisquer $\psi$ de $S u b(\varphi)$ e $s^{\prime}$ de $W^{\prime}$. A demonstração segue por indução no tamanho de $\psi$.

Para os casos em que $\psi$ é letra proposicional, tem a forma de negação ou de implicação, a demonstração é trivial, pois $W^{\prime} \subseteq W$ e $\pi_{W}^{\prime}$ é a restrição de $\pi_{W}$ a $W^{\prime}$. Analisemos o caso em que $\psi$ é da forma $K_{1} \chi$ : mostramos primeiro que se $\left(\mathrm{M}, s^{\prime}\right) \models \psi$, então $\left(\mathrm{M}^{\prime}, s^{\prime}\right) \models \psi$. Seja $s^{\prime} \in W^{\prime} \operatorname{com}\left(\mathrm{M}, s^{\prime}\right) \models K_{1} \chi$, então $(\mathrm{M}, t) \vDash \chi$, para todo $t$ de $W$ tal que $\left(s^{\prime}, t\right) \in \mathcal{K}_{1}$; em particular, vale que $\left(\mathrm{M}^{\prime}, t\right) \models \chi$, para todo $t^{\prime}$ de $W^{\prime} \operatorname{com}\left(s^{\prime}, t\right) \in \mathcal{K}_{1}^{\prime}$. Pela hipótese de indução, se $\left(\mathrm{M}^{\prime}, t\right) \models \chi$ para todo $t$ de $W^{\prime}$ tal que $\left(s^{\prime}, t\right) \in \mathcal{K}_{1}^{\prime}$, então $\left(\mathrm{M}^{\prime}, s^{\prime}\right) \vDash K_{1} \chi$.

Para a recíproca, usamos a contra-positiva, suponha $\left(\mathrm{M}, s^{\prime}\right) \not K_{1} \chi$, isto é, existe $t$ em $W$ tal que $\left(s^{\prime}, t\right) \in \mathcal{K}_{1}$ e $(\mathrm{M}, t) \vDash \neg \chi$. Desde que $\mathcal{K}_{1}$ é universal, segue que $(w, t) \in \mathcal{K}_{1}$ e $(\mathrm{M}, w) \vDash \neg K_{1} \chi$, ou seja, $K_{1} \chi \in \Gamma$ e existe $s_{\chi} \in \mathrm{M}$ tal que $\left(\mathrm{M}, s_{\chi}\right) \models \neg \chi$. Por construção, $s_{\chi} \in W^{\prime}$ e pela hipótese de indução $\left(\mathrm{M}^{\prime}, s_{\chi}\right) \models \neg \chi$. Como $\left(s^{\prime}, s_{\chi}\right) \in \mathcal{K}_{1}^{\prime}$ temos que $\left(\mathrm{M}^{\prime}, s^{\prime}\right) \not \models K \chi$.

Portanto, $\left(\mathrm{M}, s^{\prime}\right) \models \psi$ se, e só se, $\left(\mathrm{M}^{\prime}, s^{\prime}\right) \models \psi$, para toda subfórmula $\psi$ em $S u b(\varphi)$ e qualquer $s^{\prime}$ de $W^{\prime}$. Desde que $w \in W^{\prime}$ e por hipótese $(\mathrm{M}, w) \vDash \varphi$ temos que $\left(\mathrm{M}^{\prime}, w\right) \vDash \varphi$.

De modo similar podemos mostrar que:

\section{Proposição 5.7 (Halpern).}

Uma fórmula $\varphi$ é satisfativel em KD45 se, e só se, é satisfeita numa estrutura $\mathcal{M}_{1}^{\text {elt }}$ com pelo menos $|\varphi|$ mundos.

\section{Demonstração.}

A demonstração para KD45 é análoga à da proposição anterior. Novamente, sem perda de generalidade, utilizamos a estrutura $M$ sugerida pela Proposição 3.7 e construimos $\mathrm{M}^{\prime}$ que satisfaça nossas necessidades. Podemos tomar $\mathrm{M}^{\prime}$ da proposição anterior, pois $\mathrm{M}^{\prime} \in \mathcal{M}_{1}^{\text {elt }}$ (Prop. 3.2) e $\mathrm{M}^{\prime}$ satisfaz os critérios exigidos na Proposição 3.7. A satisfatibilidade segue de modo análogo (Prop. 3.1).

Com isso podemos mostrar que é possível construir um algoritmo NP para decidir a satisfatibilidade em S5. O teorema a seguir formaliza esta idéia.

Teorema 5.8 (Halpern).

O problema da satisfatibilidade de S5 (KD45) é NP-completo, e portanto o problema da validade de S5 (KD45) é co-NP-completo. 
Demonstração. [Halpern [36]]

A idéia para o algoritmo consiste basicamente em: tomar uma estrutura $\mathrm{M}$ em $\mathcal{M}_{1}^{\text {rst }}$ (resp., $\mathcal{M}_{1}^{\text {elt }}$ ) com no máximo $|\varphi|$ mundos possíveis e verificar se $\varphi$ é satisfeita em $M$ (pelas duas proposições anteriores existe tal estrutura). Escolher uma estrutura em $\mathcal{M}_{1}^{r s t}$ (resp., $\mathcal{M}_{1}^{\text {elt }}$ ), com as características mencionadas, de modo não determinístico significa fixar uma quantidade, de no máximo $|\varphi|$, mundos e atribuir valores-verdade às letras proposicionais que ocorrem em $\varphi$ em cada mundo da estrutura. Mais precisamente, dada $\varphi$ com $|\varphi|=m$, escolhemos $\mathrm{M}=\left\langle W, \pi_{W}, \mathcal{K}_{1}\right\rangle$ tal que $|W|=j(j \leq m), \mathcal{K}_{1}$ universal, e de modo que para qualquer $w \in W$ e todo $\psi \in \Phi$, se $\psi \notin S u b(\varphi)$, então $\pi_{w}(\psi)=\perp$. Como temos no máximo $m$ letras primitivas ocorrendo em $\varphi$, e no máximo $m$ mundos, então a escolha de $\mathrm{M}$ é feita na pior situação em tempo não-determinístico $O\left(m^{2}\right)$. Resta verificar o custo para checar se $\varphi$ é satisfeita em algum $w$ de M. Pela Proposição 5.1, a checagem pode ser feita deterministicamente em $O(\|\mathrm{M}\| \cdot \mid m)$, isto é, em $O\left(m^{3}\right)$. Pela proposição anterior (5.6), se $\varphi$ é satisfatível, um dos candidatos escolhidos a satisfaz (evidentemente, se $\varphi$ não é satisfatível não existe escolha possível); portanto, existe um algoritmo não determinístico $O\left(\mathrm{~m}^{3}\right)$ que decide a satisfatibilidade de $\varphi$.

Vimos nesta seção que os sistemas S5 e KD45 estão na classe $N P$-completo, no entanto a maioria dos sistemas de conhecimento pertencem à classe PSPACE como mostram os resultados da próxima seção.

\subsection{Limitantes inferiores}

Mostramos nesta seção que o problema da satisfatibilidade para a maioria dos sistemas de conhecimento é mais difícil que em $C P$, fato pelo qual se conjectura que problemas $P S P A C E$-difíceis não são solúveis em $N P$. Um motivo para que os sistemas $\mathrm{K}, \mathrm{T}$ e $\mathrm{S} 4$ não tenham as mesmas características que S5 e KD45 em termos da complexidade computacional é que:

\section{Teorema 5.9.}

Existe uma fórmula $\varphi_{m}^{\mathrm{k}}$ (resp., $\varphi_{m}^{\mathrm{T}}$ e $\varphi_{m}^{\mathrm{s4}}$ ) de tamanho $O\left(m^{2}\right)$ (resp., $O(m)$ e $O(m)$ ) que é K (resp., T e S4) satisfativel, porém qualquer estrutura em $\mathcal{M}_{1}$ (resp., $\mathcal{M}_{1}^{r}$ e $\mathcal{M}_{1}^{r t}$ ) que a satisfaz tem pelo menos $2^{m}$ mundos.

Demonstração. [Halpern [36]]

A idéia é construir fórmulas que forçam a existência de uma árvore binária, cujas folhas contenham os diferentes valores-verdade de $m$ letras proposicionais que ocorrem nas respectivas fórmulas. Assim, se a estru- 
tura tiver profundidade $m$ conterá $2^{m}$ nós no nível $m$. Vamos mostrar que a menor estrutura que satisfaz a fórmula é exponencial no número de variáveis da fórmula. Basicamente, o procedimento para a construção da fórmula $\varphi_{m}^{K}$ (resp., $\varphi_{m}^{\top}$ e $\varphi_{m}^{\mathrm{s} 4}$ ) é como segue: dado um número natural $m$, tomamos variáveis $p_{1} \ldots, p_{m}$ para montar as combinações possíveis de valores-verdade. Utilizamos um conjunto de variáveis $q_{0}, \ldots, q_{m+1}$ para marcar os níveis (profundidade) da estrutura, a partir das variáveis de marcação, o valor-verdade das variáveis $p_{j}$ é determinada pela profundidade $j$ na estrutura. Para formalizar este procedimento, utilizamos duas fórmulas básicas ( $c f$. Halpern [36] e Ladner [48]). Para $0 \leq j \leq m-1$ :

$$
\left(q_{j} \wedge \neg q_{j+1}\right) \rightarrow\left(\neg K \neg\left(q_{j+1} \wedge \neg q_{j+2} \wedge p_{j+1}\right) \wedge \neg K \neg\left(q_{j+1} \wedge \neg q_{j+2} \wedge \neg p_{j+1}\right)\right)
$$

A fórmula acima força, a partir de um mundo $w$ na profundidade $j$, a ocorrência de uma ramificação e indica a possibilidade de encontrar dois mundos sucessores de $w$ em $j+1$ tais que $p_{j+1}$ é verdadeiro em um e falso noutro. A outra fórmula está definida para $1 \leq j \leq m$ :

$$
q_{j} \rightarrow\left(\left(p_{j} \rightarrow K\left(q_{j} \rightarrow p_{j}\right)\right) \wedge\left(\neg p_{j} \rightarrow K\left(q_{j} \rightarrow \neg p_{j}\right)\right)\right)
$$

A fórmula propaga os valores-verdade de $p_{j}$ e de sua negação aos mundos sucessores, isto é, se $p_{j}$ é verdadeira (resp., falsa) em um mundo $w$ localizado em profundidade $l \operatorname{com} l \geq j$, então ela é verdadeira (resp. falsa) em todos os mundos que sucedem $w$.

As propriedades mencionadas sobre as duas fórmulas dependem diretamente das características do operador $K$ (principalmente do axioma $K$ e da Prop. 2.1). Estabelecidas as fórmulas básicas, vamos elaborar as expressões que permitem proceder a marcação dos níveis, aplicar as valorações adequadas e propagá-las até os mundos localizados na profundidade $m$. Primeiramente, o critério de marcação é feito utilizando a fórmula:

$$
\bigwedge_{j=1}^{m+1}\left(q_{j} \rightarrow q_{j-1}\right)
$$

A variável $q_{j}$ denota a profundidade a partir de $j$, note que $q_{j}$ é verdadeira em qualquer mundo de profundidade maior ou igual a $j$. Abreviamos esta fórmula por depth. Para que a ramificação e a propagação de valores-verdade valha para todos os mundos em um nível, tomamos a conjunção das respectivas fórmulas:

$$
\bigwedge_{j=0}^{m-1}\left(\left(q_{j} \wedge \neg q_{j+1}\right) \rightarrow\left(\neg K \neg\left(q_{j+1} \wedge \neg q_{j+2} \wedge p_{j+1}\right) \wedge \neg K \neg\left(q_{j+1} \wedge \neg q_{j+2} \wedge \neg p_{j+1}\right)\right)\right)
$$


e

$$
\bigwedge_{j=1}^{m}\left(q_{j} \rightarrow\left(\left(p_{j} \rightarrow K\left(q_{j} \rightarrow p_{j}\right)\right) \wedge\left(\neg p_{j} \rightarrow K\left(q_{j} \rightarrow \neg p_{j}\right)\right)\right)\right)
$$

Chamamos a primeira de branching e a outra de determined ( $c f$. Halpern [36]). Para cada caso, construimos as fórmulas que satisfazem o enunciado:

i) A fórmula $\varphi_{m}^{\mathrm{K}}$ é dada por

$$
q_{0} \wedge \neg q_{1} \wedge \bigwedge_{j=0}^{m} K^{j}(\text { depth } \wedge \text { determined } \wedge \text { branching })
$$

Notação: $K^{0} \varphi$ abrevia $\varphi$ e $K^{j+1} \varphi$ é abreviação de $K K^{j} \varphi$, para $j \geq 1$. Vemos que o tamanho de $\varphi_{m}^{\mathrm{k}}$ é $O\left(m^{2}\right)$, basta verificar que $\bigwedge_{j=0}^{m} K^{j}$ (depth $\wedge$ determined $\wedge$ branching $)$ é formado por uma conjunção da ordem de $m$ e cada termo é uma conjunção de mesma ordem.

Seja $M$ a estrutura construída de acordo com o processo de construção indicado pelas fórmulas depth, branching e determined. $\mathrm{M} \in \mathcal{M}_{1}$ e a estrutura possui pelo menos $2^{m}$ mundos. Vejamos se $M$ satisfaz $\varphi_{m}^{\mathrm{K}}$, olhando com maior detalhe as componentes de $\varphi_{m}^{k}$. A fórmula $q_{0} \wedge \neg q_{1}$ assegura que o mundo $w$ em $M$ que satisfaz $\varphi_{m}^{\mathrm{k}}$ está marcado como nível 0. A conjunção $\bigwedge_{j=0}^{m} K^{j}($ depth $\wedge$ determined $\wedge$ branching $)$ garante que em todo mundo $s$, atingível em $j$ passos (no máximo $m$ passos) a partir de $w$, vale $(\mathrm{M}, s) \vDash$ depth $\wedge$ determined $\wedge$ branching. Cada $K^{j}$ que antecede branching força $j(0 \leq j \leq m)$ sucessivos níveis de ramificação, sendo que cada ramificação "quebra" o valor-verdade das variáveis $p_{j}$. Analogamente, o operador $K^{j}$ que antecede determined induz os valores-verdades atribuidos às variáveis $p_{j}$ aos novos mundos nos níveis entre $j$ e $m$. Resumindo, branching cria as valorações e determined as preserva. Para finalizar, a partir de $w$, no nível 0 , há um caminho para cada um dos $2^{m}$ mundos no nível $m$, e como cada mundo foi construído contendo uma das $2^{m}$ valorações possíveis para as $p_{1}, \ldots, p_{m}$ variáveis, temos que $\varphi_{m}^{\kappa}$ é satisfeita em $\mathrm{M}$.

ii) Para $\varphi_{m}^{\top}$, utilizamos as mesmas fórmulas do item anterior, isto é, depth, determined e branching. No entanto, como o axioma $T$ permite que $\vdash K^{j} \varphi \rightarrow K^{j-1} \varphi$ para $j \geq 1$, que equivale à reflexividade em $\mathrm{M}$, então podemos escrever $\varphi_{m}^{\top}$ como:

$$
q_{0} \wedge \neg q_{1} \wedge K^{m}(\text { depth } \wedge \text { determined } \wedge \text { branching })
$$


É imediato que o tamanho de $\varphi_{m}^{\kappa}$ é $O(m)$. A propriedade de que $(\mathrm{M}, s) \models$ depth $\wedge$ determined $\wedge$ branching vale para todo mundo $s$, atingivel em $m$ passos a partir de $w$, é satisfeita pela reflexividade e de $K^{m}$ (depth $\wedge$ determined $\wedge$ branching $)$. De modo análogo ao item anterior, concluimos que $M \in \mathcal{M}_{1}^{r}$ e $M$ satisfaz $\varphi_{m}^{\top}$.

iii) Novamente, utilizando as fórmulas já descritas determinamos $\varphi_{m}^{54}$ como sendo:

$$
q_{0} \wedge \neg q_{1} \wedge K(\text { depth } \wedge \text { determined } \wedge \text { branching }) .
$$

É imediato que o tamanho de $\varphi_{m}^{k}$ é $O(m)$. O axioma 4 implica na transitividade em $\mathrm{M}$, que com $K($ depth $\wedge$ determined $\wedge$ branching) garante que $(\mathrm{M}, s) \vDash$ depth $\wedge$ determined $\wedge$ branching, para todo mundo $s$ atingível em $m$ passos a partir de $w$. Logo, como no item $(i), \varphi_{m}^{54}$ é satisfatível em $M \in \mathcal{M}_{1}^{r s t}$.

Sabemos que se $\varphi$ é satisfatível em $\mathrm{K}_{n}$ (resp., $\mathrm{T}_{n}, \mathrm{~S} 4_{n}, \mathrm{~S} 5_{n}$ e KD45n), então é satisfeita numa estrutura de tamanho máximo $2^{|\varphi|}$ (Teor. 3.13) e como a última proposição mostra que para K, T e S4 não podemos obter resultados melhores, temos uma suspeita de que o problema da satisfatibilidade para esses sistemas não está na classe $N P$.

\section{Observação 5.1 .}

i) Ainda, sob as condições da proposição anterior, se $\mathrm{M}=\left\langle W, \pi_{W}, \mathcal{K}\right\rangle$ é uma estrutura em $\mathcal{M}_{1}$ (resp., $\mathcal{M}_{1}^{r}$ e $\mathcal{M}_{1}^{r t}$ ) e $(\mathrm{M}, w) \models \varphi_{m}^{\mathrm{k}}$, (resp., $(\mathrm{M}, w) \vDash \varphi_{m}^{\mathrm{T}}$ e $\left.(\mathrm{M}, w) \vDash \varphi_{m}^{\mathrm{s}}\right)$, então $(\mathrm{M}, w) \models q_{0} \wedge \neg q_{1} \wedge$ branching.

ii) Supondo as condições do item anterior: se $\mathrm{M}=\left\langle W, \pi_{W}, \mathcal{K}\right\rangle$ é uma estrutura em $\mathcal{M}_{1}$ (resp., $\mathcal{M}_{1}^{r}$ e $\mathcal{M}_{1}^{r t}$ ) tal que $(\mathrm{M}, w) \vDash \varphi_{m}^{\mathrm{K}}$, (resp., $\left.(\mathrm{M}, w) \vDash \varphi_{m}^{\top} e(\mathrm{M}, w) \vDash \varphi_{m}^{54}\right)$, então existe um mundo $s$, na profundidade $j$, atíngível a partir de $w$ com $(\mathrm{M}, s) \models q_{j} \wedge \neg q_{j+1} e(\mathrm{M}, s) \models p_{l}$ se, e só $s e, \pi_{t}\left(p_{l}\right)=\mathrm{T}$ para $l=1, \ldots, j$.

Demonstramos esse fato por indução sobre $j$. Base: pelo item (i) $(\mathrm{M}, w) \vDash q_{0} \wedge \neg q_{1} \wedge$ branching, então existem $s_{0}$ e $s_{1}$ com $\left(w, s_{0}\right)$ e $\left(w, s_{1}\right)$ elementos de $\mathcal{K}$, com $s_{0}, s_{1}$ no nível $j+1 e$ $\left(\mathrm{M}, s_{0}\right) \vDash q_{0} \wedge \neg q_{1} \wedge p_{1},\left(\mathrm{M}, s_{1}\right) \models q_{0} \wedge \neg q_{1} \wedge \neg p_{1}$. Passo: seja $j<m$. Pela hipótese de indução, existe $s$ atingível a partir de $w$ tal que $(\mathrm{M}, s) \vDash q_{j} \wedge \neg q_{j+1}$ e $(\mathrm{M}, s) \models p_{l}$ se, e só $s e, \pi_{s}\left(p_{l}\right)=\mathrm{T}$ para $l=1, \ldots, j$. Como s atingivel a partir de $w$ e $\mathrm{M} \in \mathcal{M}_{1}^{r t}$, então $(\mathrm{M}, s) \models$ depth $\wedge$ determined $\wedge$ branching. A fórmula branching garante a existência de $t$ com $(s, t)$ elemento de $\mathcal{K}(t$ no nível $j+1)$ e $(\mathrm{M}, t) \models q_{j+1} \wedge \neg q_{j+2},(\mathrm{M}, t) \models p_{j+1}$ se, e somente se, $\pi_{t}\left(p_{j+1}\right)=\mathrm{T}$. 
Como depth assegura que $(\mathrm{M}, s) \vDash q_{1} \wedge \cdots \wedge q_{j}$, temos determined implicando em $(\mathrm{M}, t) \vDash p_{l}$ se, e só se, $\pi_{t}\left(p_{l}\right)=\mathrm{T}$ para $l=1, \ldots, j$. $E$ segue a tese. Para $j=m$, existem pelo menos $2^{m}$ mundos distintos em $\mathrm{M}$ onde $q_{m}$ é verdadeira.

iii) Observamos que $\varphi_{m}^{\text {s4 }}$ não é satisfativel em qualquer estrutura de $\mathcal{M}_{n}^{r s t}$. Pela Proposição 3.7 podemos considerar, sem perda de generalidade, $\mathrm{M}=\left\langle W, \pi_{W}, \mathcal{K}_{1}\right\rangle$ com $\mathcal{K}_{1}$ universal. Suponha que $\varphi_{m}^{\mathrm{s4}}$ é satisfeita em M. A fórmula branching garante a existência de dois mundos $s$ e $t$ em $\mathrm{M}$ tais que $(\mathrm{M}, s) \models q_{1} \wedge p_{1} e(\mathrm{M}, t) \models q_{1} \wedge \neg p_{1}$. Porém, como temos $(\mathrm{M}, s) \vDash$ determined, em particular $(\mathrm{M}, s) \vDash\left(q_{1} \wedge p_{1}\right) \rightarrow$ $K p_{1}$. Do fato de $\mathcal{K}$ ser universal, temos que $(s, t) \in \mathcal{K} e(\mathrm{M}, t) \vDash p_{1}$. Contradição!

O próximo resultado mostra que o problema da satisfatibilidade para a maioria dos sistemas de conhecimento é PSPACE-difícil. Como já comentamos, o problema de determinar se uma $q b f$ é verdadeira é PSPACE-completo (Observação 5.5), cf. Stockmeyer e Meyer [76]. Ainda, Ladner exibiu limitantes inferiores PSPACE para os sistemas K, T e S4 via reduções log-espaço, isto é, reduzindo o problema de decidir se uma $q b f$ é verdadeira ao problema de decisão da satisfatibilidade de fórmulas modais em $\mathrm{K}, \mathrm{T}$ e S4. A demonstração do teorema a seguir é uma variante daquela utilizada por Ladner [48] e estendida aos sistemas $\mathrm{S}_{2}$ e $\mathrm{KD}_{4} 5_{2}$. Em linhas gerais, a construção de uma fórmula modal a partir de uma dada $q b f$ é feita de modo análogo à construção da árvore quantificada da $q b f$. O processo consiste em "descascar" a $q b f$ a partir de sua leitura, da esquerda para a direita, e substituindo os quantificadores pelas valorações das letras que ocorrem em sua matriz.

\section{Teorema 5.10 (Ladner).}

i) O problema da satisfatibilidade para $\mathrm{K}, \mathrm{T}$, e S4 é PSPACE-difícil.

ii) O problema da satisfatibilidade para e $\mathrm{S}_{2}$ e $\mathrm{KD}_{4} 5_{2}$ é PSPACE-difícil.

Demonstração. [Halpern [36]]

Considere $\beta_{q b f}$ a seguinte $q b f: Q_{1} p_{1} \cdots Q_{m} p_{m} \beta\left(p_{1} \cdots p_{m}\right)$. Vamos construir uma fórmula $\varphi_{\beta_{q b f}}^{\mathrm{K}}$ (resp., $\varphi_{\beta_{q b f}}^{\mathrm{T}}, \varphi_{\beta_{q b f}}^{54}, \varphi_{\beta_{q b f}}^{55}$ e $\varphi_{\beta_{q b f}}^{\mathrm{KD}}$ ) tal que seja satisfatível em uma estrutura de $\mathcal{M}_{1}$ (resp., $\mathcal{M}_{1}^{r}, \mathcal{M}_{1}^{r t}, \mathcal{M}_{2}^{r s t}$ e $\mathcal{M}_{2}^{\text {elt }}$ ) se, e somente se, $\beta_{q b f}$ é verdadeira. Uma vez obtida cada uma das fórmulas, devemos mostrar essa equivalência. Pelo Teorema 3.8, uma fórmula é satisfatível em $\mathrm{T}$ (resp., S4, $\mathrm{S}_{2}$ e KD45 2 ) se, e só se, é satisfatível em uma estrutura de 
$\mathcal{M}_{1}^{r}$ (resp., $\mathcal{M}_{1}^{r t}, \mathcal{M}_{2}^{r s t}$ e $\mathcal{M}_{2}^{\text {elt }}$ ), portanto $\varphi_{\beta_{q b f}}^{\mathrm{T}}$, (resp., $\varphi_{\beta_{q b f}}^{54}, \varphi_{\beta_{q b f}}^{55}$ e $\varphi_{\beta_{q b f}}^{\mathrm{KD}}$ ) é satisfatível se, e somente se, $\beta_{q b f}$ é verdadeira; o mesmo vale para $\varphi_{\beta_{q b f}}^{\mathrm{k}}$. Finalmente, se a ordem do tamanho das fórmulas modais for polinomial em relação ao tamanho da $\beta_{q b f}$, então temos uma redução, e o problema da satisfatibilidade para esses sistemas de conhecimento é PSPACE-difícil.

Para cada caso, a construção é similar à da Proposição 5.9. Utilizamos as fórmulas depth e determined, porém nos restringimos às valorações que tornam $\beta_{q b f}$ verdadeira, ao invés de forçarmos todas as valorações possíveis, para isso "quebramos" a fórmula branching:

$\bigwedge_{j: Q_{j+1}=\forall}\left(\left(q_{j} \wedge \neg q_{j+1}\right) \rightarrow\left(\neg K \neg\left(q_{j+1} \wedge \neg q_{j+2} \wedge p_{j+1}\right) \wedge \neg K \neg\left(q_{j+1} \wedge \neg q_{j+2} \wedge \neg p_{j+1}\right)\right)\right)$

$\mathrm{e}$

$\bigwedge_{Q_{j+1}=3}\left(\left(q_{j} \wedge \neg q_{j+1}\right) \rightarrow\left(\neg K \neg\left(q_{j+1} \wedge \neg q_{j+2} \wedge p_{j+1}\right) \wedge \neg K \neg\left(q_{j+1} \wedge \neg q_{j+2} \wedge \neg p_{j+1}\right)\right)\right)$

A primeira fórmula simula o comportamento do quantificador universal sobre a variável $p_{j+1}$ da $q b f$, ou seja, assegura que cada mundo na profundidade $j$ ramifica em pelo menos dois outros mundos, na profundidade $j+1$, de modo que num deles a variável $p_{j+1}$ é verdadeira e noutro é falsa. Analogamente, a segunda fórmula simula o comportamento do quantificador existencial sobre $p_{j+1}$, garante que cada mundo na profundidade $j$ ramifica em pelo menos um mundo, na profundidade $j+1$. Chamamos a conjunção das fórmulas acima de branching $g_{q f f}$.

i) Iniciamos analisando o problema da satisfatibilidade para o sistema $\mathrm{K}$. A fórmula $\varphi_{\beta_{g b f}}^{\mathrm{e}}$ é dada pela conjunção:

$$
q_{0} \wedge \neg q_{1} \wedge \bigwedge_{j=0}^{m} K^{j}\left(\text { depth } \wedge \text { determined } \wedge \text { branching } g_{q b f} \wedge\left(q_{m} \rightarrow \beta\right)\right)
$$

Basicamente, a ordem do tamanho da fórmula $\varphi_{\beta_{q b f}}^{\mathrm{C}}$ continua polinomial (quadrático) em relação ao tamanho da $\beta_{q b f}$, como na proposição 5.9. Mais precisamente, note que cada uma das fórmulas da conjunção pode ser gerada utilizando-se espaço logarítmico, logo a redução de uma $q b f$ para uma fórmula modal em $\mathrm{K}$ é log-espaço o que é suficiente para afirmar que o problema da satisfatibilidade para $K$ é $P S P A C E-$ completo via log-reduções.

E como já comentamos, a construção de $\varphi_{\beta_{q b f}}^{\mathrm{K}}$ a partir de $\beta_{q b f}$ é análoga à construção da árvore quantificada da $q b f$, nesse sentido 
as fórmulas depth e determined são as que forçam a existência da árvore binária, enquanto branching $g_{q b f}$ diz como a ramificação ocorre. Diferentemente das fórmulas da Proposição 5.9 a ramificação não é sempre binária, caso o quantificador encontrado seja o existencial a extensão para o próximo nível se dá por um único ramo.

Resta verificar que $\beta_{q b f}$ é verdadeiro se, e só se, $\varphi_{\beta_{q b f}}^{K}$ é satisfatível em alguma estrutura de $\mathcal{M}_{1}$. É imediato que se $\beta_{q b f}$ é verdadeiro, então $\varphi_{\beta_{q b f}}^{K}$ é satisfatível em alguma estrutura de $\mathcal{M}_{1}$; vejamos a recíproca. Suponha $M=\left\langle W, \pi_{W}, \mathcal{K}\right\rangle$, uma estrutura em $\mathcal{M}_{1}$, tal que $(\mathrm{M}, w)=\varphi_{\beta_{q b f}}^{\mathrm{K}}$. Então temos que:

$$
\begin{aligned}
& \text { a) } \quad(\mathrm{M}, w) \models q_{0}, \\
& \text { b) } \quad(\mathrm{M}, w) \models K\left(q_{m} \rightarrow \beta\right) .
\end{aligned}
$$

Notação: dado um mundo $s$ em $\mathrm{M}$, seja $\beta_{j}^{s}$ a $q b f Q_{j+1} p_{j+1} \cdots Q_{m} p_{m} \beta$, de modo que todas as ocorrências de $p_{l}$, para $l<j$ são substituidas por 1 , se $\pi_{w}\left(p_{l}\right)=\mathrm{T}$, e 0 caso contrário. Logo, $\beta_{0}^{w}=\beta_{q b f}$ e $\beta_{m}^{w}$ é o resultado obtido a partir de $\beta$ com todas as ocorrências das variáveis $p_{j}$ substituidas por 1 ou 0 , conforme o caso. Logo, se $(w, s) \in \mathcal{K}$ temos que:

c) se $(\mathrm{M}, s) \models q_{m}$, então $\beta_{m}^{s}$ é verdadeira,

d) $\quad$ se $(\mathrm{M}, s) \models q_{m-j} \wedge \neg q_{m-j+1}$, então $\beta_{m-j}^{s}$ é verdadeira.

Os fatos em (a) são imediatos da hipótese. (b) decorre da hipótese e das propriedades de $K$ (Prop. 2.1). $\quad \mathrm{O}$ fato em (c) decorre de $(b)$ e da definição de $\beta_{m}^{s}$. Para $(d)$, basta fazer uma indução sobre $j$ : a base da indução decorre de $(c)$ e o passo é imediato; basta ver que se $\beta_{m-(j+1)}^{s}$ é falsa, então $\beta_{m}^{s}$ é falsa, item (c) e de $\bigwedge_{j=0}^{m} K^{j}\left(\right.$ depth $\wedge$ determined $\wedge$ branching $g_{q b f} \wedge\left(q_{m} \rightarrow \beta\right)$. Logo, se tomarmos $j=m$, então pelos itens $(d)$ e $(a)$ vale que $\beta_{0}^{w}=\beta_{q b f}$ é verdadeira.

A fórmula $\varphi_{\beta_{q b f}}^{\top}$ é dada por

$$
q_{0} \wedge \neg q_{1} \wedge K^{m}\left(\text { depth } \wedge \text { determined } \wedge \text { branching } g_{q b f} \wedge\left(q_{m} \rightarrow \beta\right)\right) .
$$

A análise é idêntica à do item anterior, os fatos em $(a),(b)$ e $(c)$ decorrem de modo imediato e para o fato em $(d)$ basta considerar a reflexividade de $\mathcal{K}$.

Para S4 a fórmula $\varphi_{\beta_{q b f}}^{54}$ é

$q_{0} \wedge \neg q_{1} \wedge K\left(\right.$ depth $\wedge$ determined $\wedge$ branching $\left.g_{q b f} \wedge\left(q_{m} \rightarrow \beta\right)\right)$. 
A análise é idêntica ao item anterior.

ii) A fórmula $\varphi_{\beta_{q b f}}^{\mathrm{S5}}$ é estruturalmente idêntica à $\varphi_{\beta_{q b f}}^{\top}$, porém sustituimos todas as ocorrências de $K$ por $K_{1} K_{2}$ :

$q_{0} \wedge \neg q_{1} \wedge\left(K_{1} K_{2}\right)^{m}\left(\right.$ depth $\wedge$ determined $\wedge$ branching $\left.g_{q b f} \wedge\left(q_{m} \rightarrow \beta\right)\right)$,

Vamos mostrar que se $\beta_{q b f}$ é verdadeiro, então $\varphi_{\beta_{q b f}}^{55}$ é satisfatível em alguma estrutura de $\mathcal{M}_{2}^{r s t}$. Tomamos $\mathrm{M}=\left\langle W, \pi_{W}, \mathcal{K}\right\rangle$ em $\mathcal{M}_{1}^{r t}$ com $w_{0}$ sendo o mundo no nível 0 , então $\left(\mathrm{M}, w_{0}\right) \models \varphi_{\beta_{q b f}}^{54}$. A idéia é construir uma estrutura $\mathrm{M}^{\prime}$ em $\mathcal{M}_{2}^{\text {rst }}$, a partir de $\mathrm{M}$, que satisfaça $\varphi_{\beta_{\text {qbf }}}^{55}$. Basicamente, a relação $\mathcal{K}$ é duplicada gerando $\mathcal{K}_{1}$ e $\mathcal{K}_{2}$. Seja $\mathrm{M}^{\prime}=\left\langle W^{\prime}, \pi_{W^{\prime}}^{\prime}, \mathcal{K}_{1}, \mathcal{K}_{2}\right\rangle$ com $W^{\prime}=W \cup\left\{w_{(w, s)} \mid(w, s) \in \mathcal{K}\right\}$, $\pi_{w^{\prime}}^{\prime}=\pi_{w}$ para todo $w \in W$ e $\pi_{w_{(w, s)}^{\prime}}^{\prime}=\pi_{w}$, para $w \in W, \mathcal{K}_{1}$ é o fecho reflexivo, simétrico e transitivo do conjunto de arestas $\left\{\left(w, w_{(w, s)}\right) \mid(w, s) \in \mathcal{K}\right\}$ e $\mathcal{K}_{2}$ é o fecho reflexivo, simétrico e transitivo do conjunto $\left\{\left(w_{(w, s)}, w\right) \mid(w, s) \in \mathcal{K}\right\}$.

Por construção, $\mathrm{M}^{\prime} \in \mathcal{M}_{2}^{r s t}$, então resta verificar que $\left(\mathrm{M}, w_{0}\right) \models \varphi_{\beta_{q b f}}^{55}$ se, e só se, $\beta_{q b f}$ é verdadeira. Supondo $\left(\mathrm{M}, w_{0}\right) \models \varphi_{\beta_{q b f}}^{55}$, vamos mostrar que $\beta_{q b f}$ é verdadeira. A argumentação é análoga à feita para $\varphi_{\beta_{q b f}}^{54}$, exceto para os itens $(c)$ e $(d)$. Para os fatos em $(c)$ e $(d)$, ao invés de considerarmos as arestas $(w, s) \in \mathcal{K}$, devemos olhar a relação $\mathcal{K}_{1} \circ \mathcal{K}_{2}$, ou seja, vamos considerar todas as arestas $(s, t)$ tais que existe $u$ com $(s, u) \in \mathcal{K}_{1}$ e $(u, t) \in \mathcal{K}_{2}$. Veja que os fatos em $(c)$ e $(d)$ se verificam, pois eles valem para todo $(s, u) \in \mathcal{K}_{1}$ e $(u, t) \in \mathcal{K}_{2}$. Logo, segue que $\beta_{q b f}$ é verdadeira.

Para a recíproca, consideramos a construção de $M^{\prime}\left(M^{\prime} \in \mathcal{M}_{2}^{r t}\right)$, o fato de $\beta_{q b f}$ ser verdadeiro, já que (M, $\left.w_{0}\right) \models \varphi_{\beta_{q b f}}^{\text {s4 }}$ e a Proposição 3.1. Nessas condições podemos afirmar que $\left(\mathrm{M}^{\prime}, w_{0}\right) \models \varphi_{\beta_{q b f}}^{55}$.

Finalmente, para $\mathrm{KD} 45_{2}$ a fórmula $\varphi_{\beta_{q b f}}^{\mathrm{KD} 45}$ é idêntica a $\varphi_{\beta_{q b f}}^{\mathrm{S5}}$. Como a estrutura $\mathrm{M}^{\prime}$ do item anterior é elemento de $\mathcal{M}_{2}^{\text {elt }}$, segue que $\left(\mathrm{M}, w_{0}\right) \models \varphi_{\beta_{q b f}}^{55}$ se, e só se, $\beta_{q b f}$ é verdadeira.

$\mathrm{O}$ resultado exposto sugere que o problema da satisfatibilidade para $\mathrm{S}_{2}$ e KD45 ${ }_{2}$ é significativamente mais difícil que quando consideramos um único agente nesses sistemas. E decorre de modo imediato que:

\section{Corolário 5.11.}

PSPACE é um limitante inferior para os sistemas $\mathrm{K}_{n}, \mathrm{~T}_{n}, \mathrm{~S}_{n}$ com $n \geq 1$ e S5,$K_{\mathrm{n}} 45_{\mathrm{n}}$ com $n \geq 2$. 
Mostramos nesta seção que qualquer algoritmo que solucione o problema de decidir a satisfatibilidade em $\mathrm{K}_{n}, \mathrm{~T}_{n}, \mathrm{~S} 4_{n}$ com $n \geq 1$ e S5 $5_{n}, \mathrm{KD} 45_{\mathrm{n}}$ com $n \geq 2$ é pelo menos PSPACE. Na próxima seção exibimos um limitante superior, isto é, explicitamos um algoritmo que resolva o mesmo problema, mostrando que o limitante inferior coincide com o limitante superior.

\subsection{Procedimentos de decisão usando tableau}

Inicialmente, introduzimos noções básicas relacionadas ao método de tableaux para o cálculo proposicional da lógica clássica para podermos estender seu uso para a lógica modal. Foi com Smullyan ([74] - 1968) que o método de tableaux se popularizou. O método desenvolvido por Beth (1959) possui uma variante criada por Hintikka (1955). O tableau de Beth utiliza duas árvores em sua construção, ao contrário do tableau de Hintikka que se baseia em uma única árvore. Smullyan utilizou a idéia do tableau de Beth, porém usando uma árvore em que cada nó representa uma única fórmula; diferente do tableau de Hintikka, em que um nó constitui-se de um conjunto infinito de fórmulas. $\mathrm{O}$ uso do método de tableau para decidir a satisfatibilidade de fórmulas modais foi primeiramente empregada por Kripke (1963). Em nosso caso, generalizamos as técnicas, baseadas no método de tableaux, que Ladner [48] usou para tratar a satisfatibilidade em K, T e S4.

Um tableau proposicional é um conjunto ${ }^{8} \mathbb{T}$ de fórmulas de $C P$ tal que:

i) se $\neg \neg \varphi \in \mathbb{T}$, então $\varphi \in \mathbb{T}$,

ii) se $\varphi \wedge \psi \in \mathbb{T}$, então $\varphi \in \mathbb{T}$ e $\psi \in \mathbb{T}$,

iii) se $\neg(\varphi \wedge \psi) \in \mathbb{T}$, então ou $\neg \varphi \in \mathbb{T}$ ou $\neg \psi \in \mathbb{T}$,

iv) não é o caso de ambos $\neg \varphi$ e $\varphi$ pertencerem a $\mathbb{T}$.

Dizemos que $\mathbb{T}$ é um tableau proposicional para $\varphi$ se $\mathbb{T}$ é um tableau proposicional e $\varphi \in \mathbb{T}$. E segue a primeira propriedade de $\mathbb{T}$ :

Proposição 5.12.

Uma fórmula $\varphi$ é satisfativel se e só se existir um tableau para $\varphi$.

\section{Demonstração.}

Segue da definição de consistência (Def. 2.4) e da Proposição 2.3.

\footnotetext{
${ }^{8}$ T não necessariamente é finito, porém nossas construçōes forçam sua finitude.
} 
Vamos mostrar que o tableau pode ser estendido aos sistemas de conhecimento, preservando a propriedade da proposição anterior.

Um $\mathrm{K}_{n}$-tableau é uma upla $\mathbb{T}=\left(W, l, \mathcal{K}_{1}, \ldots, \mathcal{K}_{n}\right)$ com:

- $W$ conjunto de mundos possíveis,

- $\mathcal{K}_{1}, \ldots, \mathcal{K}_{n}$ as relações de acessabilidade e

- $l$ uma função rotuladora, que associa a cada mundo $w$ de $W$ um conjunto $l(w)$ de fórmulas tal que:

1. $l(w)$ é um tableau proposicional,

2. se $K_{i} \psi \in l(w)$ e $(w, s) \in \mathcal{K}_{i}$, então $\psi \in l(s)$

3. se $\neg K_{i} \psi \in l(w)$, então existe $s \operatorname{com}(w, s) \in \mathcal{K}_{i}$ e $\neg \psi \in l(s)$.

Um $T_{n}$-tableau é um $\mathrm{K}_{n}$-tableau que satisfaz:

4. se $K_{i} \psi \in l(w)$, então $\psi \in l(w)$.

Um $S 4_{n}$-tableau é um $\mathrm{T}_{n}$-tableau que satisfaz:

5. se $K_{i} \psi \in l(w)$ e $(w, s) \in \mathcal{K}_{i}$, então $K_{i} \psi \in l(s)$.

Um $S 5_{n}$-tableau é um $T_{n}$-tableau que satisfaz:

6. se $(w, s) \in \mathcal{K}_{i}$, então $K_{i} \psi \in l(w)$ se, e só se, $K_{i} \psi \in l(s)$.

Claramente, a cláusula (6) implica a cláusula (5), logo todo $\mathrm{S} 5_{n^{-}}$

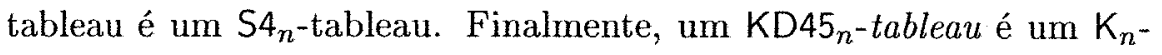
tableau que satisfaz:

7. a) se $(w, s),(w, t) \in \mathcal{K}_{i}$ e $K_{i} \psi \in l(s)$, então $K_{i} \psi \in l(t)$ e $\psi \in l(t)$,

b) se $K_{i} \psi \in l(w)$, então ou $\psi \in l(w)$, ou existe $s \operatorname{com}(w, s) \in \mathcal{K}_{i}$,

c) se $K_{i} \psi \in l(w)$ e $(w, s) \in \mathcal{K}_{i}$, então $K_{i} \psi \in l(s)$.

A cláusula $(a)$ corresponde à propriedade euclideana, $(b)$ à propriedade serial e $(c)$ à transitividade (veja que são coincidentes (7.c) e (5)). 
Dizemos que $\mathbb{T}=\left(W, l, \mathcal{K}_{1}, \ldots, \mathcal{K}_{n}\right)$ é um $\mathrm{K}_{n}$ (resp., $\left.\mathrm{T}_{n}, \mathrm{~S} 4_{n}, \mathrm{~S} 5_{n}, \mathrm{KD}_{4} 5_{n}\right)$ tableau para $\varphi$ se $\mathbb{T}$ é um $\mathrm{K}_{n}$ (resp., $\mathrm{T}_{n}, \mathrm{~S}_{n}, \mathrm{~S} 5_{n}, \mathrm{KD} 45_{n}$ ) tableau e $\varphi \in l(w)$ para algum mundo $w$ de $W$.

Por se tratar de uma árvore $\mathbb{T}$, apresenta as propriedade de uma estrutura de tipo árvore (Def. 4.11). A seguinte afirmação é verdadeira.

\section{Proposição 5.13.}

A fórmula $\varphi$ é $\mathrm{K}_{n}$ (resp., $\mathrm{T}_{n}, \mathrm{~S}_{n}, \mathrm{~S}_{n}$ e $\mathrm{KD}_{4} 5_{n}$ ) satisfativel se, e somente se, existe um tableau $\mathrm{K}_{n}$ (resp., $\mathrm{T}_{n}, \mathrm{~S}_{n}, \mathrm{~S}_{n}$ e $\mathrm{KD}_{4} 5_{n}$ ) para $\varphi$.

Demonstração. [Halpern [36]]

Primeiro, vamos mostrar que se $\varphi$ é $\mathrm{K}_{n}$ (resp., $\mathrm{T}_{n}, \mathrm{~S} 4_{n}, \mathrm{~S} 5_{n}$ e KD45 $5_{n}$ ) satisfatível, então existe um tableau $\mathrm{K}_{n}$ (resp., $\mathrm{T}_{n}, \mathrm{~S}_{n}, \mathrm{~S} 5_{n}$ e $\mathrm{KD} 45_{n}$ ) para $\varphi$. Seja $\varphi$ é satisfatível, logo existe uma estrutura $\mathrm{M}=\left\langle W, \pi_{W}, \mathcal{K}_{1}, \ldots, \mathcal{K}_{n}\right\rangle$ em $\mathcal{M}_{n}$ (resp., $\mathcal{M}_{n}^{r}, \mathcal{M}_{n}^{r t}, \mathcal{M}_{n}^{r s t}$ e $\mathcal{M}_{n}^{\text {elt }}$ ) que a satisfaz. Considere o seguinte tableau: $\mathbb{T}=\left(W, l, \mathcal{K}_{1}, \ldots, \mathcal{K}_{n}\right)$ tal que $l(w)=\{\psi \mid(\mathrm{M}, w) \vDash \psi\}$. É imediato que $\mathbb{T}$ é um tableau $\mathrm{K}_{n}$ (resp., $\mathrm{T}_{n}, \mathrm{~S}_{n}, \mathrm{~S} 5_{n}$ e $\mathrm{KD} 45_{n}$ ) para $\varphi$.

Para a recíproca, façamos primeiramente o caso da satisfatibilidade em $\mathrm{K}_{n}$. Suponha $\mathbb{T}=\left(W, l, \mathcal{K}_{1}, \ldots, \mathcal{K}_{n}\right)$ um $\mathrm{K}_{n}$-tableau para $\varphi$. Tomemos $\mathrm{M}=\left\langle W, \pi_{W}, \mathcal{K}_{1}, \ldots, \mathcal{K}_{n}\right\rangle\left(\mathrm{M} \in \mathcal{M}_{n}\right) \mathrm{com}$

$$
\pi_{w}(\varphi)= \begin{cases}T & \text { se } \varphi \in l(w) \\ \perp & \text { se } \varphi \notin l(w)\end{cases}
$$

Para mostrar que $(\mathrm{M}, w) \vDash \varphi$, basta verificar que se $\psi \in S u b(\varphi)$, então

- se $\psi \in l(w)$, então $(\mathrm{M}, w) \models \psi \quad$ e

- se $\neg \psi \in l(w)$, então $(\mathrm{M}, w) \models \neg \psi$.

A demonstração é por indução na estrutura de $\psi$. Base: se $\psi$ é letra proposicional, como $l(w)$ é um tableau proposicional (não é o caso de $\psi, \neg \psi \in l(w)$ ), então da definição de $\pi_{W}$ segue a afirmação. Passo: se $\psi$ é da forma $\neg \chi$ ou $\chi_{1} \wedge \chi_{2}$, então pela hipótese de indução de $l(w)$ ser um tableau proposicional segue o desejado. Para $\psi$ da forma $K_{i} \chi$.

- Supondo $\neg K_{i} \chi \in l(w)$, devemos mostrar que (M, $\left.w\right) \models \neg K_{i} \chi$. Pela cláusula (3) da definição de $K_{n}$ tableau, se $\neg K_{i} \chi \in l(w)$, então existe $s \operatorname{com}(w, s) \in \mathcal{K}_{i} \mathrm{e} \neg \chi \in l(s)$. Logo, da hipótese de indução, $(\mathrm{M}, s) \vDash \neg \chi$ e $(\mathrm{M}, w) \models \neg K_{i} \chi$.

- Vamos mostrar que se $K_{i} \chi \in l(w)$, então $(\mathrm{M}, w) \vDash K_{i} \chi$, isto é, que $(\mathrm{M}, s) \vdash \chi$, para todo $s$ com $(w, s) \in \mathcal{K}_{i}$. Mas, pela cláusula (2) da definição de $K_{n}$-tableau, se $K_{i} \chi \in l(w)$ e $(w, s) \in \mathcal{K}_{i}$, então $\chi \in l(s)$. Pela hipótese de indução, $(\mathrm{M}, s) \models \chi$, para todo $s$ tal que $(w, s) \in \mathcal{K}_{i}$, então $(\mathrm{M}, w) \models K_{i} \chi$. 
Portanto, $\varphi$ é $K_{n}$ satisfatível se, e só se, existe um tableau $K_{n}$ para $\varphi$.

Para $\mathrm{T}_{n}$ (resp., $\mathrm{S}_{n}, \mathrm{~S} 5_{n}$ e $\mathrm{KD} 45_{n}$ ), considere $\mathrm{M}=\left\langle W, \pi_{W}, \mathcal{K}_{1}^{\prime}, \ldots, \mathcal{K}_{n}^{\prime}\right\rangle$, com $\mathcal{K}_{i}^{\prime}$ o fecho reflexivo (resp., reflexivo e transitivo; reflexivo, simétrico e transitivo; e reflexivo, simétrico e transitivo) e $\pi_{W}$ como definido anteriormente, então $M$ é elemento de $\mathcal{M}_{n}^{r}$ (resp., $\mathcal{M}_{n}^{r t}, \mathcal{M}_{n}^{r s t}$ e $\mathcal{M}_{n}^{\text {elt }}$ ).

Resta mostrarmos que se existe um tableau $\mathrm{T}_{n}$ (resp., $\mathrm{S}_{n}, \mathrm{~S}_{n}$ e $\mathrm{KD} 45_{n}$ ) para $\varphi$, então $\varphi$ é satisfatível em $T_{n}$ (resp., $\mathrm{S} 4_{n}, \mathrm{~S} 5_{n}$ e $\mathrm{KD} 45_{n}$ ). A demonstração é análoga à feita para o caso $\mathrm{K}_{n}$. Para os casos em que $\psi$ é letra proposicional, tem a forma $\neg \chi$ ou $\chi_{1} \wedge \chi_{2}$, segue como feito anteriormente. Para $\psi$ da forma $K_{i} \chi \mathrm{e} \neg K_{i} \chi \in l(w)$, basta considerar que a cláusula (3) ocorre na definição de todos os tipos de tableaux e que $\mathcal{K}_{i} \subseteq \mathcal{K}_{i}^{\prime}$. Finalmente, o caso $K_{i} \chi \in l(w)$. Devemos mostrar que $(\mathrm{M}, w) \vDash K_{i} \chi$, isto é, que $(\mathrm{M}, s) \models \chi$, para todo $s \operatorname{com}(w, s) \in \mathcal{K}_{i}$.

- $\mathrm{T}_{n}$ : Como $\mathcal{K}_{i}^{\prime}$ é o fecho reflexivo e transitivo de $\mathcal{K}_{i}$, logo se $(w, s) \in \mathcal{K}_{i}^{\prime}$, então existe um caminho $s_{0}, s_{1}, \ldots, s_{m}$ com $s_{0}=w$ e $s_{m}=s$ e para todo $j<m$ ou $\left(s_{j}, s_{j+1}\right) \in \mathcal{K}_{i}$ ou $\left(s_{j+1}, s_{j}\right) \in \mathcal{K}_{i}$. De fato, trata-se de um caminho primitivo (Def. 4.10 e Prop. 4.14). Da hipótese $K_{i} \chi \in l(w)$, da cláusula (4) da definição de $T_{n}$-tableau e das propriedades de fecho de $\mathcal{K}_{i}^{\prime}$, decorre que $\chi \in l\left(s_{j}\right)$, para todo $j \leq m$. Da hipótese de indução, $\left(\mathrm{M}, s_{j}\right) \models \chi$, para todo $j \leq m$ tal que $\left(w, s_{j}\right) \in \mathcal{K}_{i}, \operatorname{logo}(\mathrm{M}, s) \vDash K_{i} \chi(\mathrm{em}$ particular, vale para $j=m$ ). Como isso vale para todo $s$ tal que $(w, s) \in \mathcal{K}_{i}^{\prime}$, então $(\mathrm{M}, w) \models K_{i} \chi$.

- S4n A demonstração é idêntica ao caso anterior, basta observar que, da cláusula (5) da definição de $54_{n}$-tableau, tem-se que $K_{i} \chi \in l\left(s_{j}\right)$ para todo $j \leq m$. Por fim, a cláusula (4) implica em $\chi \in l(s)$. Portanto, $(\mathrm{M}, w) \vDash K_{i} \chi$.

- $S 5_{n}$ e $K D 45_{n}$ : A demonstração para $\mathrm{S} 5_{n}$ é análoga ao caso anterior, evidentemente utilizando as respectivas cláusulas. $\mathrm{O}$ caso $\mathrm{KD} 45_{n}$ é idêntico ao $\mathrm{S} 5_{n}$, pois $\mathrm{M}$ é elemento de $\mathcal{M}_{n}^{\text {elt }}$.

Portanto, segue a tese.

Com esse resultado traçamos a seguinte estratégia: dada uma fórmula $\varphi$, elaboramos um algoritmo que força um $\mathrm{K}_{n}$-tableau para $\varphi$. Incluimos as modificações necessárias para obtermos tableau $\mathrm{T}_{n}, \mathrm{~S} 4_{n}, \mathrm{~S} 5_{n}$ e $\mathrm{KD}_{4} 5_{n}$ para $\varphi$. Para cada caso, mostramos que a construção do tableau termina e decide a satisfatibilidade de $\varphi$ se, e só se, $\varphi$ é satisfatível. Por fim, demonstramos que existe um algortimo que verifica se a construção de cada tableau é feita em espaço polinomial em $|\varphi|$.

Seja $\mathbb{T}$ um tableau; dizemos que $\mathbb{T}$ é um tableau completamente expandido 
se, para toda fórmula $\psi \in \mathbb{T}$ e subfórmula $\chi$ de $\psi$, ou $\chi \in \mathbb{T}$ ou $\neg \chi \in \mathbb{T}$. Dividimos o processo de construção de um $\mathrm{K}_{n}$-tableau em:

i) expande um conjunto $\mathbb{T}$ de fórmulas a um tableau proposicional;

ii) constrói um tableau proposicional completamente expandido;

iii) escolhe um nó, cujo rótulo é um tableau completamente expandido, e cria sucessores a partir do nó para satisfazer a cláusula (3) da definição de $\mathrm{K}_{n}$-tableaux;

iv) verifica rótulos satisfatíveis.

O processo de construção dos tableaux para os sistemas $\mathrm{T}_{n}, \mathrm{~S} 4_{n}, \mathrm{~S} 5_{n}$ e $\mathrm{KD} 45_{n}$ segue as mesmas regras; evidentemente os itens em (iii) e (iv) devem ser modificados, de acordo com cada caso. A criação de nós sucessores deve respeitar as definições que distinguem cada um dos tableaux.

Por comodidade, definimos alguns termos: evidentemente, se um conjunto de fórmulas não é um tableau proposicional, existe um elemento do conjunto que não satisfaz nenhum dos itens que define esse conjunto como tableau proposicional. Analogamente, se um conjunto de fórmulas não é completamente expandido, existe $\psi$ subformula de algum elemento do conjunto, tal que ambos $\psi \mathrm{e} \neg \psi$ são elementos do conjunto. Nas duas situações acima, a fórmula que garante que um conjunto não é um tableau proposicional ou que um conjunto não é completamente expandido recebe o nome de testemunha. Estamos supondo que as fórmulas estão ordenadas de algum modo, por exemplo, pelo tamanho. A ordenação permite escolher a menor testemunha (segundo a ordenação adotada), se existir. Dizemos que um conjunto é evidentemente inconsistente se existe $\psi$, tal que ambos $\psi \mathrm{e}$ $\neg \psi$ são elementos do conjunto.

$\mathrm{O}$ método para obter o $\mathrm{K}_{n}$-tableau para $\varphi_{0}$ :

1) Construa uma árvore com um único nó $w_{0}, \operatorname{com} l\left(w_{0}\right)=\left\{\varphi_{0}\right\}$.

2) Repita até que nenhum dos itens $(a)-(d)$ se aplique:

(a) - Montando o tableau proposicional: se $w$ é um folha da árvore, $l(w)$ não é evidentemente inconsistente, $l(w)$ não é um tableau proposicional e $\psi$ é a menor testemunha desse fato, então:

i) se $\psi$ é da forma $\neg \neg \chi$, então criar um sucessor $s$ de $w$ (isto é, adicionar um nó $s$ à árvore e uma aresta de $w$ a $s)$ e $l(s)=l(w) \cup\{\chi\}$, 
ii) se $\psi$ é da forma $\chi_{1} \wedge \chi_{2}$, então criar um sucessor $s$ de $w$ e $l(s)=l(w) \cup\left\{\chi_{1}, \chi_{2}\right\}$,

iii) se $\psi$ é da forma $\neg\left(\chi_{1} \wedge \chi_{2}\right)$, então criar dois sucessores $s_{1}$ e $s_{2}$ de $w$ e $l\left(s_{j}\right)=l(w) \cup\left\{\neg \chi_{j}\right\}$, para $j=1,2$,

(b) - Montando o tableau proposicional completamente expandido: se $w$ é um folha da árvore, $l(w)$ não é evidentemente inconsistente, $l(w)$ não é um tableau proposicional completamente expandido e $\psi$ é a menor testemunha desse fato, então criam-se dois sucessores $s^{\prime}$ e $s^{\prime \prime}$ de $w$ e $l\left(s^{\prime}\right)=l(w) \cup\{\psi\}$, $l\left(s^{\prime \prime}\right)=l(w) \cup\{\neg \psi\}$,

(c) - Criando os nós sucessores:

se $w$ é um folha da árvore, $l(w)$ não é evidentemente inconsistente, $l(w)$ não é um tableau proposicional completamente expandido, então, para cada fórmula da forma $\neg K_{i} \psi \in l(w)$, cria-se um $i$-sucessor $s$ de $w$ (isto é, adicionamos um nó $s$ à árvore e uma aresta de $w$ a $s$ rotulada por $i$ ) e $l(s)=l(w) / K_{i} \cup\{\neg \psi\}$. (Lembrando, que $l(w)$ é um conjunto de fórmulas, então $l(w) / K_{i}$ consiste de todas as fórmulas $\chi$ tal que $\left.K_{i} \chi \in l(w)\right)$.

(d) - Marcando os nós satisfativeis:

se $w$ não está marcada com SAT, então marcar $w$ com SAT se:

i) $l(w)$ não é um tableau proposicional completamente expandido e $s$ está marcado com SAT para algum sucessor $s$ de $w$, ou

ii) $l(w)$ é um tableau proposicional completamente expandido, não existem fórmulas da forma $\neg K_{i} \psi \in$ $l(w)$ e $l(w)$ não é evidentemente inconsistente, ou

iii) $l(w)$ é um tableau proposicional completamente expandido, $w$ possui sucessores e todos eles estão marcados com SAT,

3) Se a raiz está marcada com SAT, então retorna $\varphi_{0}$ é satisfatível; caso contrário retorna $\varphi_{0}$ não é satisfativel. 
Vamos simular uma construção, considere a fórmula $\varphi_{0}$ como sendo:

$$
(p \wedge \neg(p \wedge q)) \wedge\left(K_{1}(\neg p) \wedge \neg K_{1} K_{2} q\right) .
$$

Fazemos um roteiro da simulação que é complementada pela figura 5.3:

- Iniciamos criando um mundo $s_{0} \operatorname{com} l\left(s_{0}\right)=\left\{\varphi_{0}\right\}$.

Formamos um tableau proposicional a partir de $\varphi_{0}$, como temos três conjunções ocorrendo em $\varphi_{0}$, aplicamos o passo (a.ii) três vezes. $\mathrm{O}$ resultado é $s_{3}$ com:

$$
l\left(s_{3}\right)=\left\{\varphi_{0}, p \wedge \neg(p \wedge q), K_{1} \neg p \wedge \neg K_{1} K_{2} q, K_{1} \neg p, \neg K_{1} K_{2} q, p, \neg(p \wedge q)\right\} .
$$

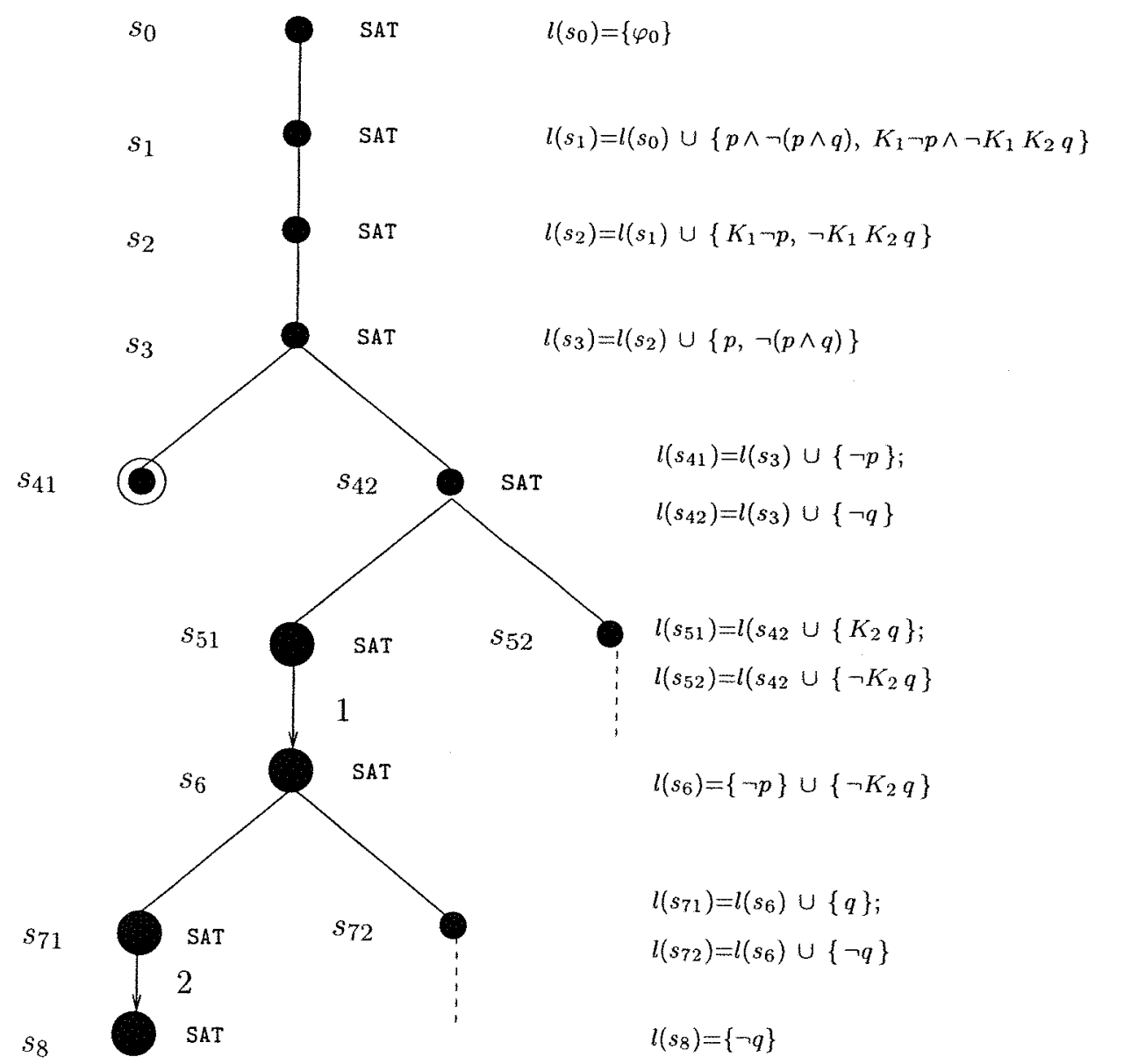

Figura 5.2: Construção do tableau $K_{n}$ para $\varphi_{0}=(p \wedge \neg(p \wedge q)) \wedge\left(K_{1} \neg p \wedge \neg K_{1} K_{2} q\right)$. 
- $l\left(s_{3}\right)$ não é um tableau proposicional e a única testemunha disso é $\neg(p \wedge q)$, pois se $\neg(p \wedge q) \in l\left(s_{3}\right)$, então $\neg p \in l\left(s_{3}\right)$ ou $\neg q \in l\left(s_{3}\right)$. Logo, aplicamos-se o passo (a.iii) e criamos dois nós sucessores $s_{41}$ e $s_{42}$ com

$$
l\left(s_{41}\right)=l\left(s_{3}\right) \cup\{\neg p\} \text { e } l\left(s_{42}\right)=l\left(s_{3}\right) \cup\{\neg q\} .
$$

- $l\left(s_{41}\right)$ é evidentemente inconsistente $\left(p \in l\left(s_{41}\right) \mathrm{e}\right.$ $\left.\neg p \in l\left(s_{41}\right)\right)$. Note que nenhum passo de $(a)-(c)$ se aplica, ou seja, nenhum nó sucessor é criado e não há como $l\left(s_{41}\right)$ ser marcado com SAT.

- $l\left(s_{42}\right)$ é um tableau proposicional e continuamos sua expansão.

- $l\left(s_{42}\right)$ não é um tableau proposicional completamente expandido e a única testemunha é $K_{2} q$. Aplicamos o passo (b), criando dois nós sucessores $s_{51}$ e $s_{52}$ com

$$
l\left(s_{51}\right)=l\left(s_{42}\right) \cup\left\{K_{2} q\right\} \text { e } l\left(s_{52}\right)=l\left(s_{42}\right) \cup\left\{\neg K_{2} q\right\} .
$$

Novamente, temos dois casos:

- $\quad l\left(s_{\overline{5} 1}\right)$, nesse momento, é uma folha que é um tableau proposicional completamente expandido e que não é evidentemente inconsistente. Nessas condições, o passo $(c)$ permite criar nós sucessores, como a única fórmula da forma $\neg K_{i} \psi$ em $l\left(s_{51}\right)$ é $\neg K_{1} K_{2} q$, criamos um 1 -sucessor $l\left(s_{6}\right)$ de $l\left(s_{51}\right)$ com

$$
l\left(s_{6}\right)=\{\neg p\} \cup\left\{\neg K_{2} q\right\} .
$$

Obs.: $\{\neg p\}=l\left(s_{51}\right) / K_{1}$.

- A situação do conjunto $l\left(s_{52}\right)$ é análoga ao $l\left(s_{51}\right)$, porém contendo duas fórmulas que permitem criar dois sucessores: $l\left(s_{61}\right)$ e $l\left(s_{62}\right) \mathrm{com}$

$$
l\left(s_{61}\right)=\{\neg p\} \cup\left\{\neg K_{2} q\right\} \text { e } l\left(s_{62}\right)=\{q\} \cup\{\neg q\} .
$$

O nó $l\left(s_{61}\right)$ é 1-sucessor e $l\left(s_{62}\right) 2$-sucessor. A análise de $l\left(s_{61}\right)$ é idêntica à de $l\left(s_{6}\right)$ e $l\left(s_{62}\right)$ é evidentemente inconsistente como $l\left(s_{41}\right)$. 
- $l\left(s_{6}\right)$ não é um tableau proposicional completamente expandido e uma testemunha desse fato é $q$. Aplicando o passo (b), criamos dois nós sucessores $l\left(s_{71}\right)$ e $l\left(s_{72}\right)$ com

$$
l\left(s_{71}\right)=\left\{\neg p, \neg K_{2} q, q\right\} \text { e } l\left(s_{72}\right)=\left\{\neg p, \neg K_{2} q, \neg q\right\} .
$$

Para cada um dos sucessores, temos:

- $l\left(s_{71}\right)$, nesse momento, é uma folha que é um tableau proposicional completamente expandido que, pelo passo $(c)$, permite criar um 2-sucessor $l\left(s_{8}\right)$, pois $\neg K_{2} q$ é a única fórmula da forma $\neg K_{i} \psi$ em $l\left(s_{71}\right)$. Temos

$$
l\left(s_{8}\right)=\{\neg q\} .
$$

Nenhum passo de $(a)-(c)$ se aplica e, como $l\left(s_{8}\right)$ é um tableau proposicional completamente expandido, que não contém fórmulas da forma $K_{i} \psi$, é marcado com SAT, pelo passo $(d)$.

- Para $l\left(s_{72}\right)$, a situação é análoga à de $l\left(s_{71}\right)$. seja $l\left(s_{81}\right)$ o 2 -sucessor de $l\left(s_{72}\right)$.

- Como já dissemos, a análise de $l\left(s_{61}\right)$ é idêntica a de $l\left(s_{6}\right)$.

- Como resultado: o passo (d.i) marca sat a todos os nós, com excessão de $l\left(s_{41}\right)$ e $l\left(s_{62}\right)$.

Ressaltamos que o método não constrói efetivamente um tableau, mas gera uma árvore que chamamos de pré-tableaux. Os nós do pré-tableau são rotulados por conjuntos de fórmulas (como num tableau) e algumas arestas são rotuladas por agentes. Apesar da figura 5.2 apresentar somente parte do pré-tableau, esta é suficiente para exibir o $\mathrm{K}_{n}$-tableau para $\varphi_{0}$, que consiste de um subconjunto dos nós do pré-tableau, aqueles cujo rótulo é um tableau proposicional completamente expandido (todos os outros nós são ignorados).

Note que o caminho de $s_{0}$ a $s_{51}$ é para a criação de um tableau completamente expandido. O mesmo é feito de $s_{6}$ a $s_{71}$. Os rótulos aplicados às arestas indicam os elementos que irão compor as relações de acessibilidade (mundos e arestas). Assim, o $\mathrm{K}_{n}$-tableau para $\varphi_{0}$ pode ser extraído do pré-tableau como sendo: $\mathbb{T}=\left(W^{\prime}, l^{\prime}, \mathcal{K}_{1}^{\prime}, \mathcal{K}_{2}^{\prime}\right) \operatorname{com} W^{\prime}=\left\{s_{51}, s_{71}, s_{8}\right\}, l^{\prime}$ é a restrição de $l$ ao conjunto $W^{\prime}, \mathcal{K}_{1}^{\prime}=\left\{\left(s_{51}, s_{71}\right)\right\}$ e $\mathcal{K}_{2}^{\prime}=\left\{\left(s_{71}, s_{8}\right)\right\}$. 
Para os resultados que se seguem, necessitamos definir alguns termos. Como fizemos antes, dado um conjunto de fórmulas $l(w)$, a fórmula $\psi_{l(w)}$ indica a conjunção de todas as fórmulas em $l(w)$. Dizemos que um nó em um pré-tableau é interno se $l(w)$ não é um tableau proposicional completamente expandido, caso contrário, chamamos o nó de estado. Dizemos que um estado $s$ é um $\mathcal{K}_{i}$-sucessor do estado $w$ se $s$ e $w$ são consecutivos ao longo de um ramo e a primeira aresta sobre o caminho entre eles é rotulado com $i$. Observe que para $s$ ser um $\mathcal{K}_{i}$-sucessor de $w$, ambos devem ser estados e deve haver um caminho $s_{0}, \ldots, s_{m}$ e tal que $s_{0}=w, s_{m}=s$ e $s_{1}$ é um $i$ sucessor de $s_{0}$, para todo $j(0<j<m), s_{j}$ é um nó interno. O aninhamento $d e p_{K}$ de operadores $K_{i}$ é definido de modo indutivo: $\operatorname{dep}_{K}(\varphi)=0$ se $\varphi$ é letra proposicional, $\operatorname{dep}_{K}(\neg \varphi)=\operatorname{dep}_{K}(\varphi), \operatorname{dep}_{K}(\varphi \wedge \psi)=\max \left\{\operatorname{dep}_{K}(\varphi), \operatorname{dep}_{K}(\psi)\right\}$ $\operatorname{e} \operatorname{dep}_{K}\left(K_{i} \varphi\right)=1+\operatorname{dep}_{K}(\varphi)$. Assim, $\operatorname{dep}_{K}(l(w))=\max \left\{\operatorname{dep}_{K}(\psi) \mid \psi \in l(w)\right\}$.

Antes de apresentarmos os próximos resultados, seguem alguns comentários sobre o método de construção:

\section{Observação 5.2 .}

a) Analisemos mais de perto o passo (2.d), isto é, a relação entre a marca SaT de um nó $w$ e a consistência ou não de $l(w)$. O que ocorre se um nó do pré-tableau que não está marcado com $\mathrm{SAT}$ ?

- Veja que a cláusula (2.d.i) trata dos nós internos, um nó interno não tem a marca SAT se, e só se, não possui sucessores marcados com SAT, veja a árvore da simulação.

- As cláusulas (2.d.ii) e (2.d.iii) tratam dos nós que não são internos. Podem ocorrer dois casos: (1) - se um nó estado w não está marcado com SAT, então w possui um sucessor que não está marcado com SAT $e$ $l(w)$ contém uma fórmula da forma $\neg K_{i} \psi$. (2) - se um nó estado $w$ não está marcado com sar, então w possui um sucessor que não está marcado com SAT el $l(w)$ é evidentemente inconsistente.

- Ainda, de (2.d.ii) se w é uma folha, então w não está marcado com sat se, e só se, $l(w)$ é evidentemente inconsistente, isto é, $\psi_{l(w)}$ é inconsistente (Def. 2.4).

- Se w não é uma folha, então w não está marcado com SAT se, e só se, nenhum sucessor de $w$ está marcado com sat. Pelo item anterior, $\psi_{l(s)}$ é inconsistente para todo s sucessor de $w$. Logo, $\psi_{l(s)}$ é inconsistente. Vejamos um exemplo: nas condiçôes determinadas, suponha que $s_{1}$ $e s_{2}$ são os sucessores de $w$, originados por $\neg\left(\chi_{1} \wedge \chi_{2}\right)$; testemunha de que $l(w)$ näo é um tableau proposicional (como ocorrido em $l\left(s_{3}\right)$ $d a$ simulação). Logo, como $l\left(s_{j}\right)=l(w) \cup\left\{\neg \chi_{j}\right\}$ para $j=1,2$ temos 
que $\vdash\left(\neg \psi_{l\left(s_{1}\right)} \wedge \neg \psi_{l\left(s_{2}\right)}\right) \rightarrow \neg \psi_{l\left(s_{1}\right)}$ (como feito no Teorema 2.6). Do item anterior, $\psi_{l\left(s_{1}\right)}$ e $\psi_{l\left(s_{1}\right)}$ são inconsistentes, isto é, $\vdash \neg \psi_{l\left(s_{j}\right)}$ para $j=1,2$. Portanto, $\vdash \neg \psi_{l(w)}$ e $\psi_{l(w)}$ é inconsistente.

b) A linha (2) do algoritmo envolve passos não-deterministicos, evidentemente a construção independe das escolhas feitas durante o processo (mostramos que ela é correta). E como já dissemos, o não-determinístico não altera a pertinência à classe PSPACE, pois PSPACE $=$ NPSPACE (cf. Savitch [72]).

c) Pela Proposição 5.9, sabemos que toda estrutura que satisfizer um fórmula $\varphi_{0}$ em $\mathrm{K}_{n}, \mathrm{~T}_{n}, \mathrm{~S}_{n}$ para $n \geq 1$, e $\mathrm{S}_{n}$ e $\mathrm{KD}_{4} 5_{n}$ para $n \geq 2$, deve possuir pelo menos um número exponencial de mundos no tamanho de $\varphi_{0}$. Este fato parece contradizer a possibilidade de existir um algoritmo que decide a satisfatibilidade de $\varphi_{0}$ usando espaço polinomial. De fato, o pré-tableau contém um número, exponencial de nós, porém:

- a baixa profundidade (no máximo polinomial em $\left|\varphi_{0}\right|$ ) permite precorrer a árvore utilizando espaço polinomial (usando, por exemplo, depthfirst-search). Veja que a profundidade de cada ramo é limitada pelo aninhamentos de operadores $K_{i}$ em $\varphi_{0}$.

- o fato do algoritmo considerar somente uma quantidade polinomial de fórmulas em cada mundo,

garantem que a representação de um ramo completo da árvore utiliza espaço polinomial. Portanto, a árvore toda usa espaço polinomial.

Para os sistemas $\mathrm{T}_{n}, \mathrm{~S} 4_{n}, \mathrm{~S} 5_{n}$ e $\mathrm{KD} 45_{n}$, é necessário alterar os itens (2.d) e (2.c), conforme o caso. A maioria das modificaçôes segue a idéia da Proposição 3.12 e são tais que preservam a característica da limitação dos ramos. Vamos às modificações para obtermos tableaux para $\mathrm{T}_{n}, \mathrm{~S} 4_{n}, \mathrm{~S} 5_{n} \mathrm{e}$ $\mathrm{KD} 45_{n}$ :

- Para $T_{n}$, consideramos o método para $\mathrm{K}_{n}$. A única modificação ocorre no passo $(2 . d)$ :

$w$ não é marcado com sat se $K_{i} \psi \in l(w)$ e $\neg \psi \in l(w)$, para algum $\psi$ e $i$.

- Para $S 4_{n}$, consideramos o método para $\mathrm{T}_{n}$. Substituímos (2.c) por $\left(2 . c^{\prime}\right)$ :

se $w$ é um folha da árvore e $l(w)$ é um tableau completamente expandido, então, para cada fórmula da forma $\neg K_{i} \psi \in l(w)$, 
tomamos $l^{\prime}(w, \psi)=\left\{K_{i} \chi \mid K_{i} \chi \in l(w)\right\} \cup\{\neg \psi\}$. Se não existe nenhum ancestral $s$ de $w$ tal que $l(s)=l^{\prime}(w, \psi)$, então cria-se um $i$-sucessor $t$ de $w \operatorname{com} l(t)=l^{\prime}(w, \psi)$.

- Para $S 5_{n}$, consideramos o método para $\mathrm{T}_{n}$ e trocamos (2.c) por $\left(2 . c^{\prime \prime}\right)$ :

se $w$ é um folha da árvore e $l(w)$ é um tableau completamente expandido, então para cada fórmula da forma $\neg K_{i} \psi \in l(w)$ : $l^{\prime \prime}(w, \psi)=\left\{K_{i} \chi \mid K_{i} \chi \in l(w)\right\} \cup\left\{\neg K_{i} \chi \mid \neg K_{i} \chi \in l(w)\right\} \cup\{\neg \psi\}$. Se não existe nenhum ancestral $s$ de $w$ tal que $l(s)=l^{\prime \prime}(w, \psi)$, então cria-se um $i$-sucessor $t$ de $w \operatorname{com} l(t)=l^{\prime \prime}(w, \psi)$.

- Para $\mathrm{KD} 45_{n}$, usamos o método para $T_{n}$ e trocamos (2.c) por $\left(2 . c^{\prime \prime \prime}\right)$ :

se $w$ é um folha da árvore e $l(w)$ é um tableau completamente expandido, então para cada fórmula da forma $\neg K_{i} \psi \in l(w)$ : $l^{\prime \prime \prime}(w, \psi)=\left\{K_{i} \chi \mid K_{i} \chi \in l(w)\right\} \cup\left\{\neg K_{i} \chi \mid \neg K_{i} \chi \in l(w)\right\} \cup$ $l(w) / K_{i} \cup\{\neg \psi\}$. Se não existe nenhum ancestral $s$ de $w$ tal que $l(s)=l^{\prime \prime \prime}(w, \psi)$, então cria-se um $i$-sucessor $t$ de $w$ com $l(t)=l^{\prime \prime \prime}(w, \psi)$.

\section{Observação 5.3 .}

d) A cláusula (2.c) juntamente com o axioma $T$ garantem a reflexividade (Prop. 3.12). A cláusula (2.d) na forma original contraria o axioma $T$, pois permite que um mundo $w$ seja marcado com SAT se $K_{i} \psi \in l(w)$ e $\neg \psi \in l(w)$ para algum $\psi$ e i. A inclusão do novo item corrige este incômodo e preserva todas as propriedades anteriores (item (a) da Observação 5.2).

e) Analogamente, a substituição de (2.c) por $\left(2 . c^{\prime}\right)$ e (2.c.") visa, na criação de nós sucessores, contemplar as propriedades da transitividade e simetria, respectivamente.

f) Veja que a criação de nós sucessores de s pelo passo (2.c') ocorre pelas condições dos antecessores de s. O passo $\left(2 . c^{\prime}\right)$ preserva a transitividade $e$ não permite que $\operatorname{dep}_{K}\left(l\left(s^{\prime}\right)\right) \geq \operatorname{dep}_{K}(l(s))$, para nenhum sucessor $s^{\prime}$ de $s$.

O passo (2.c') trata de nós estados de modo que se $l(s)$ é um tableau proposicional completamente expandido, $s^{\prime}$ um $i$-sucessor e $s^{\prime \prime}$ um descendente qualquer de $s^{\prime}$, então $\operatorname{dep}_{K}(\psi)<\operatorname{dep}_{K}(l(s))$, para qualquer $\psi \in l\left(s^{\prime \prime}\right)$, tal que $\psi$ não é da forma $K_{i} \chi$. Ou seja, as únicas fórmulas cuja profundidade (aninhamentos de $K_{i}$ ) não são limitadas por $\operatorname{dep}_{K}(l(s)$ ) säo as da forma $K_{i} \chi$. Logo, se $s, s^{\prime}$ e $s^{\prime \prime}$ são estados, tais que $s^{\prime}$ sendo um $\mathcal{K}_{i}$-sucessor de $s$ e $s^{\prime \prime}$ sendo um $\mathcal{K}_{j}$-sucessor de $s^{\prime}$ com $i \neq j$, entäo $\operatorname{dep}_{K}\left(l\left(s^{\prime \prime}\right)\right)<\operatorname{dep}_{K}(l(s))$. Uma observação final sobre $\left(2 . c^{\prime}\right)$ : considere um caminho em que todas 
as arestas pertençam a algum $\mathcal{K}_{i}$, se $s^{\prime}$ é descendente de $s$ nesse caminho, então $l(s) / K_{i} \subseteq l\left(s^{\prime}\right) / K_{i}$.

g) O passo (2.c') modifica $\left(2 . c^{\prime}\right)$ para que todo i-sucessor de um estado seja rotulado com todas as fórmulas da forma $K_{i} \psi$ e $\neg K_{i} \psi$ que ocorrem no rótulo de seu predecessor. Ainda, dado um caminho em que todas as arestas pertençam a algum $\mathcal{K}_{i}$, se $s^{\prime}$ é descendente de $s$ nesse caminho, então $l(s) / K_{i}=l\left(s^{\prime}\right) / K_{i}$.

h) O passo (2.c' $\left.c^{\prime \prime \prime}\right)$ estabelece as propriedades transitiva, euclideana e serial, para KD45 $n$ (Prop. 3.12).

Finalmente, vamos mostrar que todos os processos de construção terminam.

Lema 5.14. Para qualquer fórmula $\varphi$, a construção do tableau $\mathrm{K}_{n}$ (resp., $\mathrm{T}_{n}, \mathrm{~S} 4_{n}, \mathrm{~S}_{n}$ e $\mathrm{KD}_{4}$ ) para $\varphi$ termina.

Demonstração. [Halpern [36]]

i) Iniciamos estudando o processo da construção do tableau $K_{n}$ para $\varphi$. Seja $|\varphi|=m$. A construção do $K_{n}$-tableau garante que, para qualquer nó $s$ da árvore, o rótulo $l(s)$ consiste somente das fórmulas em $S u b\urcorner(\varphi)$, $\operatorname{logo}|l(s)| \leq 2 \cdot m$ (Def. 3.8). Ainda, o número máximo que podemos aplicar os passos (2.a) e (2.b) é $m$ vezes, antes de atingirmos um nó $s^{\prime}$, tal que $s^{\prime}$ seja um estado, ou $l\left(s^{\prime}\right)$ seja evidentemente inconsistente. Pelo passo (2.c) temos que se $l(s)$ é um estado, então $d e p_{K}\left(l\left(s^{\prime}\right)\right)<$ $\operatorname{dep}_{K}(l(s))$, para qualquer sucessor $s^{\prime}$ de $s$. Desde que $\operatorname{dep}_{K}(\varphi) \leq m$, então a árvore final tem no máximo profundidade $m^{2}$. Portanto, é finito.

ii) A demonstração é idêntica à do caso anterior, pois a modificação feita para o $\mathrm{T}_{n}$-tableau não altera nenhum dos fatos afirmados no item anterior.

iii) A análise do processo de construção do $S 4_{n}$-tableau é análoga à do $\mathrm{K}_{n}$-tableau até os passos $(2 . a)$ e $(2 . b)$, isto é, se $|\varphi|=m$, então a quantidade de fórmulas $\psi$ manipuladas é da ordem de $m$. O restante da análise concentra-se no passo $\left(2 . c^{\prime}\right)$. A criação de nós sucessores pelo passo $\left(2 . c^{\prime}\right)$ ocorre sobre estados e cada sucessor $s^{\prime}$ de $s$ é determinado por $l^{\prime}(s, \psi)$, já que a existência ou não de $s^{\prime}$ depende se $s$ não possui um ancestral $s^{\prime \prime} \operatorname{com} l\left(s^{\prime \prime}=l^{\prime}(s, \psi)\right.$. Logo, devemos determinar a quantidade máxima de estados consecutivos num ramo e o número de conjuntos do tipo $l^{\prime}(s, \psi)$, para $s$ estado. Se $s^{\prime}$ é descendente de $s$ num caminho em que todas as arestas pertencem a algum $\mathcal{K}_{i}$, então 
$l(s) / K_{i} \subseteq l\left(s^{\prime}\right) / K_{i}$. Portanto, no máximo temos $m$ conjuntos distintos da forma $l(s) / K_{i}$, para $s$ nesse caminho. Como temos até $m$ fórmulas $\psi$, então podemos ter no máximo $m^{2}$ conjuntos distintos do tipo $l^{\prime}(s, \psi)$, para $s$ nesse caminho. Das observações em 5.3, temos que, para todo $i$, um ramo pode ter, na pior situação, $m^{2}$ estados consecutivos, cada qual um $\mathcal{K}_{2}$-sucessor de seu predecessor. Portanto, a profundidade máxima da árvore é da ordem de $m^{4}$.

iv) A análise da construção do $S 5_{n}$-tableau é análoga à do $S 4_{n}$-tableau. Porém, como, pelo passo $\left(2 . c^{\prime \prime}\right)$, todo $i$-sucessor de um estado é rotulado com todas as fórmulas da forma $K_{i} \psi$ e $\neg K_{i} \psi$ que ocorrem no rótulo de seu predecessor, então, se $s^{\prime}$ é descendente de $s$ num caminho em que todas as arestas pertençam a algum $\mathcal{K}_{i}$, temos que $l(s) / K_{i}=l\left(s^{\prime}\right) / K_{i}$. A simetria diminui o número de arestas para $m$, ao invés de $m^{2}$, como no caso anterior. Portanto, a profundidade máxima da árvore é da ordem de $m^{3}$.

v) Finalmente, a construção do $\mathrm{KD} 45_{n}$-tableau é análoga à do $\mathrm{S} 5_{n^{-}}$ tableau. Novamente, temos que todo $i$-sucessor de um estado é rotulado com todas as fórmulas da forma $K_{i} \psi$ e $\neg K_{i} \psi$ que ocorrem no rótulo de seu predecessor, então, se $s^{\prime}$ é descendente de $s$ num caminho em que todas as arestas pertençam a algum $\mathcal{K}_{i}$, temos que $l(s) / K_{i}=l\left(s^{\prime}\right) / K_{i}$ e $l(s) / K_{i} \subseteq l\left(s^{\prime}\right)$. Portanto, a profundidade máxima da árvore é da ordem de $m^{3}$.

O próximo teorema fornece uma forma alternativa de mostrar a completude dos sistemas de conhecimento. Suponha que $\varphi$ seja válida. Para mostrar que $\varphi$ possui uma prova, aplicamos a construção do tableau para $\neg \varphi$. Devemos ter que a raiz da árvore do pré-tableau não marca SAт (caso contrário, como mostra o teorema a seguir, $\neg \varphi$ seria satisfatível e contradiz a validade de $\varphi$ ). Portanto, do teorema a seguir, temos que $\neg \neg \varphi$ possui uma prova, logo $\varphi$ também.

\section{Teorema 5.15.}

Uma fórmula $\varphi$ é $\mathrm{K}_{n}$ (resp., $\mathrm{T}_{n}, \mathrm{~S}_{n}, \mathrm{~S}_{n}$ e $\mathrm{KD}_{4} 5_{n}$ ) satisfatível se, e somente se, a construção do tableau $\mathrm{K}_{n}\left(\right.$ resp., $\mathrm{T}_{n}, \mathrm{~S}_{n}, \mathrm{~S}_{n}$ e $\mathrm{KD}_{4} 5_{n}$ ) para $\varphi$ retorna que $\varphi$ é satisfatível.

Demonstração. [Halpern [36]]

Vamos tratar primeiramente, o caso $\mathrm{K}_{n}$.

i) Se a construção do $K_{n}$-tableau para $\varphi$ retorna que $\varphi$ é satisfativel, então $\varphi$ é $K_{n}$-satisfatível. $O K_{n}$-tableau para $\varphi$ é obtido do pré-tableau 
construído conforme anteriormente exposto. Os nós que formam o $\mathrm{K}_{n}$ tableau consistem de estados do pré-tableau marcados com sat. Consideramos $(w, s) \in \mathcal{K}_{i}$ no tableau, se $s$ é um $\mathcal{K}_{i}$-sucessor de $w$. Para constatar que a construção garante que este é um $\kappa_{n}$-tableau para $\varphi$, basta ver que:

- $l(s)$ é um tableau proposicional, pois são estados;

- se $K_{i} \psi \in l(s)$ e $(s, t) \in \mathcal{K}_{i}$, então $\psi \in l(t)$. De fato, $t$ é um $\mathcal{K}_{i}$-sucessor de $s$ e $l(t)=l(s) / K_{i} \cup\{\neg \psi\}$ (passo (2.c));

- se $\neg K_{i} \psi \in l(s)$, então existe $t$, tal que $(s, t) \in \mathcal{K}_{i}$ e $\neg \psi \in l(t)$. Imediato do passo (2.c): $l(t)=l(s) / K_{i} \cup\{\neg \psi\}$.

Ainda, existe $w$, tal que $l(w)$ é um tableau proposicional, $\operatorname{com} \varphi \in l(w)$, caso contrário, a raiz do pré-tableau não poderia estar marcada com sat. Pela Proposição 5.13, $\varphi$ é satisfatível se, e só se, existe um tableau para $\varphi$, logo $\varphi$ é $K_{n}$ satisfatível.

A recíproca: se $\varphi$ é $\mathrm{K}_{n}$ satisfatível, então a construção do $K_{n}$-tableau para $\varphi$ retorna que $\varphi$ é satisfatível. Vamos mostrar que se um nó $w$ do pré-tableau não está marcado com SAT, então $\vdash \neg \psi_{l(w)}$ em $\mathrm{K}_{n}$, isto é, $\psi_{l(w)}$ é inconsistente. Como estamos tomando $w$, um nó qualquer do pré-tableau, em particular, se $w$ é a raiz, então temos que $\vdash \neg \varphi$ em $\mathrm{K}_{n}$. Porém, do teorema da correção, segue que $\varphi$ não é satisfatível e vale a tese.

Usamos indução no comprimento do maior caminho de $w$ até uma folha do pré-tableau para mostrar que se $w$ não está marcado com sAT, então $\vdash \neg \psi_{l(w)}$ em $K_{n}$. Base: suponha que $w$ é folha, então pelo passo $(2 . d), w$ não está marcado com sat se, e só se, $l(w)$ é evidentemente inconsistente. Nesse caso, é imediato que $\psi_{l(w)}$ é inconsistente. Passo: suponha que $w$ é um nó interno, então pelo passo $(2 . d), w$ não está marcado com SAT se, e só se, não há sucessores de $w$ marcados com SAT. Da hipótese de indução, $\psi_{l(s)}$ é inconsistente, para todo $s$ sucessor de $w$ no pré-tableau. Evidentemente, $\psi_{l(w)}$ é inconsistente (Observação 5.2). Para o caso em que não é um nó interno: $w$ não está marcado com SAT e $l(w)$ é um estado, então, pelo passo (2.d), segue que algum sucessor $s$ de $w$ no pré-tableau não está marcado com SAT. Por construção, existe $\neg K_{i} \psi \in l(w)$, tal que $l(s)=l(w) / K_{i} \cup\{\neg \psi\}$. Da hipótese de indução, $\psi_{l(s)}$ é inconsistente. Seja $l(w) / K_{i}=\left\{\chi_{1}, \ldots, \chi_{m}\right\}$, então $l(s)=\left\{\chi_{1}, \ldots, \chi_{m}, \neg \psi\right\}$. Mas $\psi_{l(s)}$ é inconsistente, então

$$
\vdash_{\kappa_{n}} K_{i} \varphi_{1} \rightarrow\left(K_{i} \varphi_{2} \rightarrow\left(K_{i} \varphi_{3} \rightarrow \cdots \rightarrow\left(K_{i} \varphi_{j} \rightarrow K_{i} \psi\right) \cdots\right)\right)
$$

(decorre de modo idêntico como feito no Teorema 2.6). Como $\left\{K_{i} \chi_{\mathrm{I}}, \ldots, K_{i} \chi_{m}, \neg K_{i} \psi\right\} \subseteq l(w)$, então $\psi_{l(w)}$ é inconsistente. 
ii) A construção do $T_{n}$-tableau é idêntica à do $K_{n}$-tableau. É imediato, da alteração feita no passo (2.d), que o tableau obtido é um $T_{n}$-tableau. A demonstração de que a construção do $T_{n}$-tableau para $\varphi$ retorna que $\varphi$ é satisfatível, então $\varphi$ é $T_{n}$-satisfatível, é idêntica ao item anterior. Novamente, para a recíproca, desejamos mostrar que se um nó $w$ do pré-tableau não está marcado com SAT, então $\psi_{l(w)}$ é $T_{n}$-inconsistente. Segue o mesmo esquema da demonstração anterior, pois a criação de nós sucessores segue método idêntico e a marcação dos nós em nada se altera (item $(d)$ Observação 5.3).

iii) A construção do $54_{n}$-tableau é análoga à do $\mathrm{K}_{n}$-tableau. $\mathrm{O} S 4_{n}$-tableau para $\varphi$ é obtido do pré-tableau construído conforme as regras anteriormente estabelecidas. Os nós que formam o $54_{n}$-tableau consistem de estados do pré-tableau marcados com sat. Porém, consideramos $(w, s) \in \mathcal{K}_{i}$ no tableau se $s$ é um $\mathcal{K}_{i}$-sucessor de $w$, ou se $s$ é o primeiro estado de um caminho que tem início com um ancestral $s^{\prime}$ de $w$, tal que $l\left(s^{\prime}\right)=l^{\prime}(w, \psi)$, para alguma fórmula $\neg K_{i} \psi \in l(w)$. O tableau obtido é um $S 4_{n}$-tableau, temos dois casos: para o caso em que se $(s, t) \in \mathcal{K}_{i}$ e $t$ é um $\mathcal{K}_{i}$-sucessor de $s$, os fatos decorrem do passo (2.c $\left.c^{\prime}\right)$. Por outro lado, se $(s, t) \in \mathcal{K}_{i}$ e $t$ é o primeiro estado de um caminho que tem início com um ancestral $s^{\prime}$ de $s$, tal que $l\left(s^{\prime}\right)=l^{\prime}(s, \psi)$, para alguma fórmula $\neg K_{i} \psi \in l(s)$, então:

- se $K_{i} \psi \in l(s)$ e $(s, t) \in \mathcal{K}_{i}$, então $\psi \in l(t)$. De fato, decorre de $\left(2 . c^{\prime}\right)$ e do antecessor $s^{\prime}$ de $s$ ser tal que $l\left(s^{\prime}\right)=l^{\prime}(s, \psi)$, para alguma fórmula $\neg K_{i} \psi \in l(s)$.

- se $\neg K_{i} \psi \in l(s)$, então existe $t$, tal que $(s, t) \in \mathcal{K}_{i}$ e $\neg \psi \in l(t)$. Análogo ao item anterior.

- se $K_{i} \psi \in l(s)$ e $(s, t) \in \mathcal{K}_{i}$, então $K_{i} \psi \in l(t)$. De $t$ ser um $\mathcal{K}_{i}$ sucessor de $s$ segue que $l(s) / K_{i} \subseteq l(t) / K_{i}$ (item $(f)$ Observação $5.3)$.

Portanto, trata-se de um $\$ 4_{n}$-tableau para $\varphi$ que, se retorna $\varphi$ é satisfativel, então $\varphi$ é $54_{n}$-satisfativel (a demonstração é idêntica à feita para o caso $K_{n}$ ). Resta mostrar que se um nó $w$ do pré-tableau não está marcado com SAT, então $\psi_{l(w)}$ é $54_{n}$-inconsistente.

Mostramos que se $w$ não está marcado com SaT, então $\vdash \neg \psi_{l(w)}$ em $\mathrm{K}_{n}$. Fazemos por indução no comprimento do maior caminho de $w$ até uma folha do pré-tableau. Para o caso em $w$ é folha, é imediato. $O$ passo indutivo segue de modo idêntico ao do item $(i)$, pois o passo (2.d) possui as mesmas características. 
iv) A construção do $55_{n}$-tableau é idêntica à montagem do $\mathrm{K}_{n}$-tableau, logo tudo segue conforme a demonstração já dada, desde que mostremos que o tableau obtido é, de fato, um $55_{n}$-tableau. Para isso, basta verificarmos que: se $(s, t) \in \mathcal{K}_{i}$, então $\psi \in l(s)$ se, e só se, $\psi \in l(t)$.

Por construção, como os nós do tableau são estados, basta mostrar que se $t$ é um $i$-sucessor de $s$, então $s$ e $t$ coincidem em todas as fórmulas da forma $K_{i} \chi$ e $\neg K_{i} \chi$. Suponha que $s^{\prime}$ é um $i$-sucessor de $s$ sobre o caminho de $s$ a $t$. O passo $\left(2 . c^{\prime \prime}\right)$ da construção garante que $l(s) \mathrm{e}$ $l\left(s^{\prime}\right)$ coincidem em todas as fórmulas da forma $K_{i} \chi$ e $\neg K_{i} \chi$. Vamos mostrar que $l(s)$ e $l(t)$ também coincidem em tais fórmulas. De fato, se $K_{i} \chi \in l(s)$, então, por construção, $K_{i} \chi \in l\left(s^{\prime}\right)$. Como $l\left(s^{\prime}\right) \subseteq l(t)$, então $K_{i} \chi \in l(t)$. De modo análogo, decorre que se $\neg K_{i} \chi \in l(s)$, então $\neg K_{i} \chi \in l(t)$. Por outro lado, suponha $K_{i} \chi \in l(t)$, logo, por construção, $K_{i} \chi$ é subfórmula de alguma fórmula $\psi \in l(s)$. De $l(s)$ ser um tableau proposicional completamente expandido, temos que $K_{i} \chi \in l(s)$ ou $\neg K_{i} \chi \in l(s)$. Porém, como vimos, se $\neg K_{i} \chi \in l(s)$, então $\neg K_{i} \chi \in l(t), \operatorname{logo} l(t)$ é evidentemente inconsistente e $l(t)$ não é um estado. Portanto $K_{i} \chi \in l(s)$. Analogamente, segue que se $\neg K_{i} \chi \in l(t)$, então $\neg K_{i} \chi \in l(s)$.

v) Finalmente, a construção do $K D 45_{n}$-tableau é idêntica à do $S 4_{n}$-tableau. Tudo segue conforme a demonstração já dada, desde que mostremos que o tableau obtido é, de fato, um $\mathrm{KD}^{4} 5_{n}$-tableau. Para isso, basta verificarmos os itens da cláusula (7): o item (a) segue de modo idêntico ao que foi feito na análise do $55_{n}$-tableau. A subcláusula (7.b) é imediata do passo (2.c' $\left.c^{\prime \prime \prime}\right)$, e a cláusula em (7.c) é a mesma em (5).

Com esse resultado, podemos mostrar que:

\section{Teorema 5.16.}

Existe um algoritmo PSPACE que decide a satisfatibilidade de $\varphi$ em $\mathrm{K}_{n}$ (resp., $\mathrm{T}_{n}, \mathrm{~S}_{n}, \mathrm{~S}_{n}$ e $\mathrm{KD}_{4} 5_{n}$ ).

Demonstração. [Halpern [36]]

De acordo com os resultados anteriores, basta exibir um algoritmo PSPACE que verifica se a raiz da árvore de construção do tableau para $\varphi$ está marcada com sat. Como a marca de um nó $w$ depende exclusivamente da marca de seus sucessores e do rótulo $l(w)$, podemos pensar em fazer uma busca por profundidade no pré-tableau. Para a proceder a busca, necessitamos de uma cadeia (ou string) de $2 \cdots m$ bits para representar o rótulo de 
qualquer nó $s$ : enumeramos todas as fórmulas em $S u b\urcorner(\varphi)(S u b\urcorner(\varphi) \leq 2 \cdot m)$ em alguma ordem (p.ex,. pelo tamanho) e a $j$-ésima fórmula na enumeração ocorre no rótulo $l(s)$ se, e só se, o $j$-ésimo bit na cadeia é 1 . Ainda, precisamos de mais $m$ bits $(S u b(\varphi) \leq m)$ para marcar os nós que necessitamos percorrer. Logo, são necessários pelo menos $3 \cdot m$ bits para a busca.

i) Para o caso $K_{n}$, é suficiente mostrar, que dado um nó $w$ de rótulo $l(w)$, se a construção do tableau começa com o nó rotulado por $l(w)$ e termina com uma árvore de altura $h$, então podemos determinar a marca de $w$ usando no máximo $(3 \cdot h+1) \cdot m$ bits. Fazemos a demonstração por indução sobre $h$. Base: $h=0$, então, ou $l(w)$ é evidentemente inconsistente, e nesse caso $w$ não está marcado com sat, ou $w$ é um estado e $l(w)$ não contém nenhuma fórmula da forma $\neg K_{i} \psi$, e nesse caso $w$ está marcado com sat. Portanto, para $h=0$ é decidível. Passo: $h>0$. Se $w$ não é um nó interno, então a construção cria um ou dois sucessores; ainda $w$ está marcado com SAT se, e só se, seus sucessores também estão (item (a), Observação 5.2). Da hipótese de indução, computamos como cada sucessor está marcado reutilizando o espaço após cada computação. Por reutilizar o espaço entendemos apagar todas as informações sobre a sub-árvore cuja raiz é um sucessor. Se $w$ não é um nó estado, a análise é similar (passo (2.d.i)).

Logo, esse o processo gasta $O(m)$ e como a profundidade máxima da árvore é quadrática em $m$ (Lema 5.14), então computamos a satisfatibilidade de $\varphi$ usando $O\left(\mathrm{~m}^{3}\right)$

ii) $\mathrm{O}$ caso $T_{n}$ é idêntico ao item anterior.

iii) Como a criação de nós sucessores (passo $\left(2 . c^{\prime}\right)$ ) de $w$ no tableau $S 4_{n}$ depende dos rótulos dos predecessores de $w$, então necessitamos verificar os rótulos dos ancestrais de $w$. Ou seja, precisamos mostrar que se $w$ é um nó de altura $h$ e $X$ é uma lista dos rótulos que aparecem nos ancestrais de $w$, então, se a construção do tableau começa com o nó rotulado por $l(w)$ e pelos rótulos em $X$, então podemos determinar a marca de $w$ usando no máximo $(2 \cdot h+3) \cdot m+O(1)+|X|$ bits.

A indução sobre $h$ é exatamente como feita anteriormente, exceto pelo espaço da reutilização para prosseguir a busca. Para finalizar, como um nó tem no máximo $m^{4}$ ancestrais (lema 5.14) e cada rótulo requer $2 \cdot m$ bits, então $|X| \leq 2 \cdot m^{5}$. Do lema 5.14 , a altura máxima da árvore é $m^{4}$, portanto podemos decidir a satisfatibilidade de $\varphi$ usando $O\left(m^{5}\right)$ bits.

iv) Os casos $\mathrm{S}_{n}$ e $\mathrm{KD} 45_{n}$ são exatamente como o item anterior, pois 
o passo que cria os nós sucessores, apesar de não serem idênticos, funcionam da mesma forma.

Todos os resultados (proposições e teoremas) apresentados podem ser resumidos na seguinte afirmação:

\section{Teorema 5.17.}

O problema da satisfatibilidade para $\mathrm{K}_{n}, \mathrm{~T}_{n}, \mathrm{~S}_{n}$ com $n \geq 1$, e $\mathrm{S}_{n}$, $\mathrm{KD}_{4}$ p para $n \geq 2$ é PSPACE-completo.

Vimos que a maioria dos sistemas de conhecimento é PSPACE-completo e que um dos fatores cruciais para a obtenção de PSPACE como limitante superior é a profundidade de cada ramo ser limitada pela profundidade $\left(d e p_{K}\right)$ da fórmula considerada. Essa característica é perdida ao acrecentarmos à linguagem maior poder de expressividade, como, por exemplo, a inclusão do operador conhecimento comum.

\subsection{Decisão envolvendo conhecimento comum}

O operador de conhecimento comum acrescenta aos sistemas de conhecimento um ganho de expressividade, sua semântica (Prop. 4.1) permite considerar afirmações genéricas, ela é verdadeira para todos os mundos atingíveis a partir do mundo referencial. A conseqüência disso é que o problema da validade para linguagens com conhecimento comum é EXPTIME-completo.

Lembrando que, dada uma fórmula $\varphi$ num sistema cuja linguagem não possui o operador de conhecimento comum, o passo chave para obtermos procedimentos de decisão PSPACE é a construção de um pré-tableau $\mathbb{T}$ de profundidade polinomial e cujo rótulo da raiz é $\varphi$. Então, a partir do tableau para $\varphi$ imerso em $\mathbb{T}$, construímos uma estrutura $M$ que satisfaça $\varphi$. Assim, temos que se $\varphi$ é satisfatível, existe uma estrutura $M$, com caminhos de comprimento máximo polinomial em $|\varphi|$, satisfazendo $\varphi$. O teorema a seguir mostra que tableaux limitados a espaço polinomial não funcionam para tratar conhecimento comum.

\section{Teorema 5.18.}

Para qualquer $m$, existe uma fórmula $\sigma_{m}^{\mathrm{K}}$ (resp., $\left.\sigma_{m}^{\top}, \sigma_{m}^{54}, \sigma_{m}^{55}, \sigma_{m}^{\mathrm{KD} 45}\right) d e$ tamanho $O\left(m^{2}\right)$ que é $\mathrm{K}^{C}$ (resp., $\mathrm{T}^{C}, \mathrm{~S}_{2}^{C}, \mathrm{~S}_{2}^{C}, \mathrm{KD}_{4}{ }_{2}^{C}$ ) satisfativel, mas toda estrutura em $\mathcal{M}_{1}$ (resp., $\mathcal{M}_{1}^{r}, \mathcal{M}_{2}^{r t}, \mathcal{M}_{2}^{r s t}, \mathcal{M}_{2}^{\text {elt }}$ ) que satisfaz $\sigma_{m}^{K}$ (resp., $\left.\sigma_{m}^{\top}, \sigma_{m}^{54}, \sigma_{m}^{55}, \sigma_{m}^{\mathrm{KD} 45}\right)$ tem caminho de comprimento $2^{m}-1$. 
Demonstração. [Halpern [36]]

Como nas demonstrações da seção 5.4 , utilizamos $m$ variáveis proposicionais para construirmos fórmulas que se satisfaçam em estruturas com pelo menos $2^{m}$ mundos. Porém, a idéia é aproveitar a característica do operador $C_{G}$, que permite pensar em fórmulas de tamanho $O\left(\mathrm{~m}^{2}\right)$ que forçam qualquer estrutura que as satisfaçam, a ter caminhos de comprimento $2^{m}-1$.

Novamente, utilizamos $p_{0}, p_{1}, \ldots, p_{m-1}, m$ variáveis proposicionais, porém o truque agora é codificar o fato de $p_{j}$ ser verdadeiro em um mundo $w$ através de um contador binário de $m$ posições. Cada uma dessas proposições codifica um bit de um contador, $p_{0}$ codifica o bit de mais baixa ordem e $p_{m}-1$ o bit da mais alta ordem. A interpretação para $p_{j}=1$ ou $p_{j}=0$ é óbvia: $p_{j}$ é verdadeiro em $w$ se, e só se, o $j$-ésimo do contador marcar 1 .

Vejamos um exemplo de dois mundos consecutivos: considere $c=c_{m-1} \cdots c_{0}$ e $d=d_{m-1} \cdots d_{0}$, dois contadores de $m$-bits, então vejamos um exemplo de $d=c+1(d$ sucede $c)$ :

\begin{tabular}{lllllllllll}
1 & 1 & 0 & 1 & 0 & 0 & 1 & 1 & 1 & 1 & $c$ \\
0 & 0 & 0 & 0 & 0 & 0 & 0 & 0 & 0 & 1 & 1 \\
\hline 11 & 0 & 0 & 0 & 0 & 0 & 0 & 0 & 0 & 0 & $d=c+1$ \\
$l$ & & & & & & &
\end{tabular}

Figura 5.3: Descrição do funcionamento do contador binário

Mais precisamente, $d=c+1$ quando:

- ou $d_{j}=0$ e $c_{j}=1$, para $0 \leq j \leq m-1$ (início da contagem em 0 )

- ou, para algum $l \leq m-1$, temos:

- $c_{j}=1$, para todo $j<l$,

- $c_{l}=0$ e $d_{j}=0$, para todo $j<l$

- $d_{l}=1$ e $c_{k}=d_{k}$, para $l+1 \leq k \leq m-1$.

A estratégia é escrever uma fórmula que força o contador a varrer os valores de 0 a $2^{m}-1$ consecutivamente na seqüência dos mundos ( $c f$. Halpern e Vardi [34, lema 4.1]). Para cada caso de $\mathrm{K}^{C}$ (resp., $\mathrm{T}^{C}, \mathrm{~S} 4_{2}^{C}, \mathrm{~S} 5_{2}^{C}, \mathrm{KD} 45_{2}^{C}$ ) nomeamos essa fórmula por: $\sigma_{m}^{\mathrm{K}}$ (resp., $\sigma_{m}^{\top}, \sigma_{m}^{54}, \sigma_{m}^{55}, \sigma_{m}^{\mathrm{KO45}}$ ).

Analisemos primeiramente o caso de $\mathrm{K}^{C}$, lembrando que, como estamos tratando de um único agente, $C_{\{1\}}=C$. Grosso modo, desejamos uma fórmula $\sigma_{m}^{\mathrm{k}}$ que é satisfeita numa estrutura $\mathrm{M}=\left\langle W, \pi_{W}, \mathcal{K}\right\rangle$ tal que $W=$ $\left\{w_{0}, w_{1}, \ldots, w_{2^{m}-1}\right\}$ e $\pi_{w_{j}}$ é definido de acordo com a codificação do valor $j$. Vejamos como determinar $\sigma_{m}^{\mathrm{k}}$, que é satisfeita de modo que garanta a existência da seqüência $w_{0}, w_{1}, \ldots, w_{2^{m}-1}$, isto é, $\left(w_{j}, w_{j+1}\right) \in \mathcal{K}$ e tal que 
os valores de $p_{0}, p_{1}, \ldots, p_{m-1}$ codificam o valor de $j$ no mundo $w_{j}$.

A fórmula $\sigma_{m}^{K}$ é obtida pela conjunção das fórmulas $\sigma_{m 1}^{K}, \sigma_{m 2}^{K}, \sigma_{m 3}^{K}$ e $\sigma_{m 4}^{K}$. A fórmula

$$
\sigma_{m 1}^{K}: C(\neg K \neg \top)
$$

garante a existência da seqüência $w_{0}, w_{1}, \ldots, w_{2^{m}-1}$, de $2^{m}-1$ mundos, não necessariamente distintos, tal que $\left(w_{j}, w_{j+1}\right) \in \mathcal{K}$.

Para assegurar que os $2^{m}$ mundos são todos distintos e que os valores de $p_{0}, p_{1}, \ldots, p_{m-1}$ codificam o valor de $j$ no mundo $w_{j}$, definimos as seguintes fórmulas:

$$
\begin{gathered}
\sigma_{m 2}^{K}: \neg p_{0} \wedge \cdots \wedge \neg p_{m-1}, \\
\sigma_{m 3}^{K}: \bigwedge_{j=0}^{m \sim 1} C\left(\left(\bigwedge_{l=0}^{j-1} p_{l}\right) \rightarrow\left(\left(p_{j} \rightarrow K \neg p_{j}\right) \wedge\left(\neg p_{j} \rightarrow K p_{j}\right)\right)\right),
\end{gathered}
$$

$\mathrm{e}$

$$
\sigma_{m 4}^{K}: \bigwedge_{j=0}^{m-1} C\left(\left(\bigvee_{l=0}^{j-1} \neg p_{l}\right) \rightarrow\left(\left(p_{j} \rightarrow K p_{j}\right) \wedge\left(p_{j} \rightarrow K \neg p_{j}\right)\right)\right)
$$

$\sigma_{m 2}^{K}$ garante que $w_{0}$ codifica o valor 0 . As fórmulas $\sigma_{m 3}^{K}$ e $\sigma_{m 4}^{K}$ certificam que se $s$ é atingível a partir de $w_{0}$ e $(s, t) \in \mathcal{K}$, então $p_{j}$ tem o mesmo valor verdade em $s$ e $t$ se, e só se, existe $p_{l}$ falso para $l<j$. Ou seja, se $s$ é atingível a partir de $w_{0}$ e $(s, t) \in \mathcal{K}$, então $s$ e $t$ codificam valores consecutivos do contador.

Por indução em $j$, é fácil ver que $w_{j}$ codifica o valor $j$. Portanto, $w_{0}, w_{1}, \ldots, w_{2^{m}-1}$ são todos distintos. Para finalizar a construção de $\mathrm{M}$, definimos a relação de acessibilidade: $\mathcal{K}=\left\{\left(w_{j}, w_{j+1}\right) \mid j<2^{m}-1\right\} \cup$ $\left\{\left(w_{2^{m}-1}, w_{0}\right)\right\}$.

Observamos que $\sigma_{m}^{\mathrm{K}}$ não é satisfeita em estruturas cuja relação de acessibilidade é reflexiva. De fato, se $\sigma_{m}^{k}$ é verdadeira em $w_{0}$, então qualquer seqüência de mundos $w_{0}, w_{1}, \ldots, w_{2^{m}-1}, \operatorname{com}\left(w_{j}, w_{j+1}\right) \in \mathcal{K}$, temos que $w_{j}$ codifica o valor $j$, e as seqüências devem ter todos os $2^{m}-1$ mundos distintos. No entanto, em estruturas reflexivas, podemos ter a seqüência $w_{0}, w_{0}, \ldots, w_{0}$ cujos elementos não são distintos.

Logo, para $\sigma_{m}^{\top}$, devemos providenciar algumas modificações, contornamos o problema introduzindo uma nova variável proposicional $p_{\Lambda}$ para marcar o fato de que uma alteração ocorreu, por exemplo, podemos considerar $p_{\Lambda}$ verdadeiro em $w_{j}$ se $j$ é par e falso em $w_{j}$ se $j$ ímpar. Assim, a idéia é construir $\sigma_{m}^{\top}$ de modo que seja verdadeira em $w_{0}$ se, em qualquer seqüência $w_{0}, w_{1}, \ldots, w_{2^{m}-1}$, tal que $\left(w_{j}, w_{j+1}\right) \in \mathcal{K}$ e $p_{\Lambda}$ alterna os valores-verdade entre mundos consecutivos nesta seqüência, então $w_{j}$ codifica o valor de $j$. 
Formalmente, $\sigma_{m}^{T}$ é a conjunção das fórmulas $\sigma_{m 1}^{T}, \sigma_{m 2}^{T}, \sigma_{m 3}^{T}$ e $\sigma_{m 4}^{T}$ descritas como segue:

$$
\begin{aligned}
\sigma_{m 1}^{T}: & C\left(\left(p_{\Lambda} \rightarrow \neg K p_{\Lambda}\right) \wedge\left(\neg p_{\Lambda} \rightarrow \neg K \neg p_{\Lambda}\right)\right), \\
\sigma_{m 2}^{T}: & \left(p_{0} \wedge \cdots \wedge p_{m-1}\right), \\
\sigma_{m 3}^{T}: \bigwedge_{j=0}^{m-1} C\left(\left(\bigwedge_{l=0}^{j-1} p_{l}\right) \rightarrow\right. & \left(\left(\left(p_{\Lambda} \wedge p_{j}\right) \rightarrow K\left(\neg p_{\Lambda} \rightarrow \neg p_{j}\right)\right) \wedge\right. \\
& \left(\left(p_{\Lambda} \wedge \neg p_{j}\right) \rightarrow K\left(\neg p_{\Lambda} \rightarrow p_{j}\right)\right) \wedge \\
& \left(\left(\neg p_{\Lambda} \wedge p_{j}\right) \rightarrow K\left(p_{\Lambda} \rightarrow \neg p_{j}\right)\right) \wedge \\
& \left.\left.\left(\left(\neg p_{\Lambda} \wedge \neg p_{j}\right) \rightarrow K\left(p_{\Lambda} \rightarrow p_{j}\right)\right)\right)\right)
\end{aligned}
$$

$\mathrm{e}$

$$
\begin{aligned}
\sigma_{m 4}^{T}: \bigvee_{j=0}^{m-1} C\left(\left(\bigvee_{l=0}^{j-1} \neg p_{l}\right) \rightarrow\right. & \left(\left(\left(p_{\Lambda} \wedge p_{j}\right) \rightarrow K\left(\neg p_{\Lambda} \rightarrow p_{j}\right)\right) \wedge\right. \\
& \left(\left(p_{\Lambda} \wedge \neg p_{j}\right) \rightarrow K\left(\neg p_{\Lambda} \rightarrow \neg p_{j}\right)\right) \wedge \\
& \left(\left(\neg p_{\Lambda} \wedge p_{j}\right) \rightarrow K\left(p_{\Lambda} \rightarrow p_{j}\right)\right) \wedge \\
& \left.\left.\left(\left(\neg p_{\Lambda} \wedge \neg p_{j}\right) \rightarrow K\left(p_{\Lambda} \rightarrow \neg p_{j}\right)\right)\right)\right) .
\end{aligned}
$$

A fórmula $\sigma_{m}^{\top}$ não é satisfeita em estruturas transitivas. Seja uma seqüência $w_{0}, w_{1}, \ldots, w_{2^{m}-1}$, tal que $\left(w_{j}, w_{j+1}\right) \in \mathcal{K}$, como caracterizada anteriormente. Logo, $w_{0}$ e $w_{2}$ codificam 0 e 2 , respectivamente. Pela transitividade, $\left(w_{0}, w_{2}\right) \in \mathcal{K}$. Porém, por $\sigma_{m 3}^{T}$ e $\sigma_{m 4}^{T}$, temos que $w_{2}$ codifica 1, contradizendo o fato de $w_{2}$ codificar 2 .

Para contornar esse problema nos casos S4, S5 e KD45, devemos considerar dois agentes. As fórmulas $\sigma_{m}^{54}, \sigma_{m}^{55} \mathrm{e} \sigma_{m}^{\mathrm{KD} 45}$ são obtidas substituindo todas as ocorrências de $K$ em $\sigma_{m}^{\mathrm{T}}$ por $K_{1} K_{2}$.

Esse resultado ratifica o que comentamos anteriormente, as estratégias utilizadas para obtermos limitantes superiores PSPACE não são suficientes para tratar sistemas envolvendo conhecimento comum.

O próximo teorema é uma variante do resultado obtido por Fischer e Ladner [29] sobre o PDL. Eles mostraram que EXPTIME é um limitante inferior para problema da satisfatibilidade do PDL. Não fazemos a demonstração propriamente dita, porém estabelecemos a relação entre os sistemas de conhecimento e o PDL. 


\section{Teorema 5.19.}

$O$ problema da satisfatibilidade para $\mathrm{K}_{n}^{C}, \mathrm{~T}_{n}^{C}$ com $n \geq 1$ e $\mathrm{S}_{n}^{C}, \mathrm{~S}_{n}^{C}$, $\mathrm{KD}_{4}{ }_{n}^{C}$ com $n \geq 2$ é EXPTIME-difícil.

\section{Observação 5.4 (Halpern).}

Apresentamos o PDL e mostramos as semelhanças com os sistemas de conhecimento considerados. Grosso modo, o PDL é uma lógica modal sobre programas. $O$ alfabeto do $\mathrm{PDL}$ consiste de uma classe de programas primitivos. Programas complexos são construídas a partir dos operadores "; $"$ " $"$ *". Por exemplo, se $\alpha$ e $\beta$ são programas, então $\alpha ; \beta$ e $\alpha^{*}$ também são. Intuitivamente, $\alpha ; \beta$ corresponde a: executar $\alpha$ e então executar $\beta$; $e$ a leitura de $\alpha^{*}$ corresponde a: executar $\alpha$ um número finito (arbitrário) de vezes. Associado a cada programa, temos o operador modal []: se $\varphi$ é uma fórmula e $\alpha$ um programa, então $[\alpha] \varphi$ é uma fórmula. Utiliza-se o operador $\langle\alpha\rangle$ como abreviação de $\neg[\neg \alpha]$.

Adota-se a semântica de Kripke para o PDL. Dado um conjunto de estados, a relação binária $\rho$ indica a transição de um estado a outro por meio de um programa, isto é, para cada programa, está associada uma relação sobre o conjunto de estados. Se $\alpha$ é um programa, então $(w, s) \in \rho(\alpha)$ significa que: se executar o programa $\alpha$ começando no estado $w$, então é possível finalizar no estado $s$. A fórmula $[\alpha] \varphi$ é verdadeira em um estado $w$ se $\varphi$ é verdadeira em todos os estados s tal que $(w, s) \in \rho(\alpha)$. A analogia com a fórmula $K \varphi$ é imediata. Definimos $\rho\left(\alpha^{*}\right)$ como o fecho transitivo de $\rho(\alpha)$, logo $\alpha^{*}$ desempenha a mesma relação com $\alpha$ que o operador $C$ com $E$.

Fischer e Ladner ([48, sec. 4]) exibiram o limitante inferior para o PDL, mostrando que para cada MT exponencial e uma entrada $x$, existe uma fórmula $\varphi_{\mathrm{MT}, x}$ do $\mathrm{PDL}$ tal que o tamanho da fórmula é $O(|x|)$ e $\varphi_{\mathrm{MT}, x}$ é satisfativel se, e somente se, MT aceita $x$. Originalmente, Fischer e Ladner utilizaram máquinas 9 "alternating" PSPACE, porém as linguagens aceitas por tais máquinas sâo exatamente as mesmas de uma MT exponencial (cf. Stockmeyer [9]). Os únicos operadores modais na fórmula $\varphi_{\mathrm{Mr}, x}$ são $[\mapsto] e$ $\left[\vdash^{*}\right]$, com $\vdash$ representando um programa primitivo.

Seja $\varphi_{\mathrm{MT}, x}^{\mathrm{K}}$ a fórmula obtida substituindo todas as ocorrências de $[H]$ em $\varphi_{\mathrm{MT}, x}$ por $K$ e todas as ocorrências de $\left[\mathbb{1}^{*}\right]$ por $C$, então $\varphi_{\mathrm{MT}, x}^{\mathrm{K}}$ é satisfativel em $\mathrm{K}^{C}$ se, e somente se, MT aceita $x$. Isso mostra que EXPTIME é um limitante inferior para $\mathrm{K}^{C}$. Para que EXPTIME seja o limitante inferior de todos os outros sistemas de conhecimento comum, basta modificar $\varphi_{\mu \mathrm{r}, x}^{\mathrm{K}}$ conforme as alterações feitas em $\sigma_{m}^{\mathrm{k}}$. Para $\mathrm{T}^{C}$, é necessário uma nova proposição primitiva $p_{\Lambda}$ para marcar a ocorrência de mudança. Assim, $\varphi_{M \mathrm{~T}, x}^{\mathrm{T}}$ é satis-

\footnotetext{
${ }^{9}$ Os detalhes da MT utilizada por Fischer e Ladner encontram-se em [48, sec, 4] e o funcionamento da máquina está no lema 4.2 .
} 
fativel em $\mathrm{K}^{C}$ se, e somente se, $\mathrm{MT}$ aceita $x$. Para $\mathrm{S}^{C}, \mathrm{~S}^{C}$ e $\mathrm{KD}^{\circ} 5^{C}$ basta substituir as ocorrências de $K$ em $\varphi_{\mathrm{MT}, x}^{\mathrm{T}}$ por $K_{1} K_{2}$.

Para finalizar a caracterização dos sistemas de conhecimento envolvendo conhecimento comum de acordo com a classe de complexidade a que pertencem, devemos exibir limitantes superiores para compará-los aos limitantes inferiores obtidos. Formalizamos isso através do próximo teorema.

\section{Teorema 5.20.}

$O$ problema da satisfatibilidade para $\mathrm{K}_{n}^{C}, \mathrm{~T}_{n}^{C} \operatorname{com} n \geq 1$ e $\mathrm{S}_{n}^{C}, \mathrm{~S}_{n}^{C}$, $\mathrm{KD} 45_{n}^{C}$ com $n \geq 2$ é EXPTIME-completo.

Demonstração. [Halpern [36]]

A demonstração segue as técnicas devidas a Pratt [68] e Fischer e Immerman [28]. Dada uma fórmula $\varphi$ num sistema envolvendo conhecimento comum, desejamos construir uma estrutura que satisfaça $\varphi$, ou mostrar que ela não existe. Boa parte da estratégia adotada para a construção segue aquela da Proposição 4.6, utilizada para mostrar a completude dos sistemas de conhecimento comum (Teor. 4.8); a modificação está no uso do conjunto $\operatorname{Con}_{C}(\varphi)$. Como não sabemos utilizar $\operatorname{Con}_{C}(\varphi)$ de modo eficiente, computacionalmente falando, definimos o conjunto $\Gamma_{\varphi}^{1}$. Os elementos de $\Gamma_{\varphi}^{1}$ são subconjuntos maximais de $S u b_{C}^{\neg}(\varphi)$ que são tableaux proposicionais. $\Sigma \in \Gamma_{\varphi}^{1}$ se, e só se, $\Sigma$ é um tableau proposicional e para qualquer $\psi \in S u b_{C}(\varphi)$ ou $\psi \in \Sigma$ ou $\neg \psi \in \Sigma$. O conjunto $\Gamma_{\varphi}^{1}$ faz papel similar da $\operatorname{Con}{ }_{C}(\varphi)$.

Note que a quantidade de elementos em $\Sigma \in \Gamma_{\varphi}^{1}$ não excede $2^{3 \cdot|\varphi|}$ (Prop. 4.4). Vamos construir uma seqüência $M^{j}=\left\langle W^{j}, \pi_{W}^{j}, \mathcal{K}_{1}^{j}, \ldots, \mathcal{K}_{n}^{j}\right\rangle$, para $j=1,2,3, \ldots$, com $\Gamma_{\varphi}^{1}=W^{1} \supseteq W^{2} \supseteq \cdots$. Suponha definidos os mundos possíveis, então definimos as relações de acessibilidade e as valorações do seguinte modo: $\mathcal{K}_{i}^{j}=\left\{(w, s) \mid w, s \in W^{j}\right.$ e $\left.w / K_{i} \cup w / E_{G} s\right\}$, para $i \in G$ e $\pi_{w}^{j}(\psi)=T$ se, e somente se, $\psi \in w$.

Determinando os conjuntos $W^{j}$. Dizemos que $w$ em $W^{j}$ é consistente se:

- para toda fórmula $\neg K_{i} \psi \in w$, existe $s$ em $W^{j}$ tal que $(w, s) \in \bigcup_{i \in G} \mathcal{K}_{i}$ com $\neg \psi \in s$,

- para toda fórmula $\neg E_{G} \psi \in w$, existe $s$ em $W^{j}$ tal que $(w, s) \in \bigcup_{i \in G} \mathcal{K}_{i}$ com $\rightarrow \psi \in s$,

- para toda fórmula $\neg C_{G} \psi \in w$, existe $s$ em $W^{j}$ atingível a partir de $w$ tal que $\neg \psi \in s$.

Se cada mundo de $W^{j}$ é consistente e $\varphi \in w$ para algum $w$ em $W^{j}$, então $\varphi$ é satisfativel. Se não existe $w$ em $W^{j}$ que seja consistente e com $\varphi \in w$, 
então $\varphi$ não é satisfatível. Caso contrário, seja $W^{j+1}$ o conjunto de todos os conjuntos consistentes em $W^{j}$ e continuamos a construção das $\mathrm{M}^{j}$.

Como $\Gamma_{\varphi}^{1}$ contém no máximo $2^{3 \cdot|\varphi|}$ elementos e $W^{j} \supseteq W^{j+1}$, então a construção termina no máximo em um número exponencial de passos. A verificação da consistência de cada mundo de $W^{j}$ pode ser feita em tempo polinomial no tamanho de $W^{j}$, que, no máximo, é exponencial no tamanho de $\varphi$. Portanto, a construção completa consome tempo exponencial.

Veja que a satisfatibilidade de $\varphi$ decorre do fato de que: se todos os mundos em $W^{j}$ são consistentes, então para quaisquer $w \in W^{j}$ e $\psi \in S u b_{C}(\varphi)$, $(\mathrm{M}, w) \models \psi$ se, e só se, $\psi \in w$. A demonstração é por indução na estrutura de $\psi$, idêntica àquela feita no Teorema 4.7. Por outro lado, se $w$ é inconsistente, então $\varphi_{w}$, a conjunção de todas as fórmulas em $w$, é inconsistente. Logo, se não existir $w \in W^{j}$ tal que $\varphi \in w$, então $\varphi$ é inconsistente e portanto não é satisfatível. Novamente, a demonstração desse fato é análoga à do teorema 4.7. Portanto, o algoritmo é correto.

Pelo teorema acima, o limitante superior e o inferior são os mesmos, logo a caracterização dos sistemas de conhecimento comum está completa.

Nosso algoritmo constrói de modo determinístico uma estrutura que satisfaz a fórmula dada, porém podemos elaborar de modo relativamente simples um algoritmo EXPTIME não-determinístico: dada $\varphi$ uma fórmula num sistema envolvendo conhecimento comum, se $\varphi$ é satisfatível, então pelo teorema em 4.7 ela o é numa estrutura de $n$ agentes e cujo tamanho não excede $2^{(3+n) \cdot|\varphi|}$ (Prop. 4.4). Como $n$ é uma constante, esse valor nos fornece um limitante superior não-determinístico EXPTIME. Para verificar a satisfatibilidade de $\varphi$ basta tomarmos uma estrutura de modo não-determinístico, dentre as $2^{(3+n) \cdot|\varphi|}$ estruturas, e fazer a checagem (Prop. 5.1); nesse caso o tempo da checagem é ótimo.

Resumidamente, tratamos dois problemas computacionais associados à lógica modal: o problema do model-checking e o da validade. Vimos que ambos são decidíveis e, particularmente, que o problema da validade para a maioria dos sistemas é PSPACE-completo. O que é um tanto quanto surpreendente, tendo em conta que os sistemas considerados, a despeito de sua sintaxe proposicional, é essencialmente uma linguagem da lógica de primeira ordem (LPO) (Observação 3.2). Leve-se em conta que os problemas mencionados para a LPO são problemas computacionalmente difíceis (a indecidibilidade da LPO é robusta). Isso reforça nosso interesse no estudo da complexidade computacional dos sistemas modais, em suas várias formas. 



\section{Referências Bibliográficas}

[1] A. Avron. Simple consequence relations. Information and Computation, 92(1):105-139, 1991.

[2] P. Blackburn, M. de Rijke, and Y. Venema. Modal Logic, volume 53 of Cambridge Tracts in Theoretical Computer Science. Cambridge University Press, 1999. Draft Version.

[3] G. Boolos. The Logic of Provability. Cambridge University Press, 1993.

[4] D. Bostok. Intermediate Logic. Clarendon Press., 1997. Cap.: 5.

[5] R. Bull and K. Segerberg. Basic modal logic. In D. Gabbay and F. Guenthner, editors, Handbook of Philosophical Logic. Vol. II: Extensions of Classic Logic., pages 167-247. D. Reidel Publishing Co., Dordrecht, 1984.

[6] W. Carnielli and C. Pizzi. Modalitá e Multimodalitá. Franco Angeli, 2001.

[7] B. Carpenter. Type-Logical Semantics. MIT Press, 1997.

[8] L. F. del. Cerro and M. Penttonen. A note on the complexity of the satisfability of modal horn clauses. J. of Logic Programming, 4:1-10, 1987.

[9] Kozen D. Chandra, A. K. and L. J. Stockmeyer. Alternation. Journal of the ACM, 28:114-133, 1981.

[10] C. C. Chang and H. J. Keisler. Model Theory. North-Holland, 3 edition, 1990. 
[11] B. F. Chellas. Modal Logic: An Introduction. Cambridge University Press, 1980.

[12] M. L. D. Chiara. Quantum logic. In D. Gabbay and F. Guenthner, editors, Handbook of Philosophical Logic. Vol. III: Alternatives to Classical Logic, pages 427-469. D. Reidel Publishing Co., Dordrecht, 1986. Cap.: III.7.

[13] A. Church. A formulation of the simple theory of types. Journal of Symbolic Logic, 5:56-68, 1940.

[14] S. A. Cook. The complexity of theorem proving procedures. In Proceedings of 3rd ACM Symposium on Theory of Computing, pages 151-158. ACM, 1971.

[15] N. C. A. da Costa. O Conhecimento Científico. Discurso Editorial, 1997.

[16] V. Costa. A two-dimensional modal logic for knowledge representation in asynchronous multi-agent systems.

[17] M. Cresswell and Hugues G. An Introduction to Modal Logic. Muthuen, 1968.

[18] R. Diestel. Graph Theory, volume 173 of Graduate Texts in Mathematics. Springer-Verlag, second edition, 2001.

[19] H. N. Duc. On the epistemic foundations of agent theories: A position paper. Technical report, Australian National University, Camberra, ACT 0200, Australia, 1995.

[20] J. M. Dunn. A truth value semantics for modal logic. In Truth, Syntax and Modality. Proceedings of the Temple University Conference on Alternative Semantics. Edited by Hugues Leblanc, volume 68 of Studies in Logic and the Foundations of Mathematics, pages 87-100. NorthHolland Publishing Co., 1973.

[21] E. A. Emerson. Temporal and modal logics. In J. van Leewen, editor, Handbook of Theoretical Computer Science. Vol. B: Formal Models and Semantics, pages 995-1072. Elsevier, 1990.

[22] R. Fagin, J. Y. Halpern, and M. Y. Vardi. What can machine know? on properties of knowledge in distributed systems. Journal of the ACM, $39: 328-376,1992$. 
[23] R. Fagin, J. Y. Halpern, and M. Y. Vardi. What is a inference rule? Journal of Symbolic Logic, 57(3):1018-1045, 1992.

[24] R. Fagin, J. Y. Halpern, Moses Y., and M. Y. Vardi. Reasoning About Knowledge. The MIT Press, 1995.

[25] R. Fagin and M. Y. Vardi. An internal semantics for modal logic: Preliminary report. Technical report, University of Cambridge, 1985.

[26] R. Fagin and M. Y. Vardi. Knowledge and implicit knowledge in a distributed environment: Preliminary report. In Theoretical Aspects of Reasoning About Knowledge. Proceedings of the 1986 Conference Edited by Joseph Y. Halpern, 1986.

[27] K. Fine. An incomplete logic containing s4. Theoria, 40:23-28, 1974.

[28] M. J. Fischer and N. Immerman. Foundations of knowledge for distributed systems. In Theoretical Aspects of Reasoning About Knowledge. Proceedings of the 1986 Conference - Edited by Joseph Y. Halpern, 1986.

[29] M. J. Fischer and R. E. Ladner. Propositional dynamic logic of regular programs. Journal of Computer Science, 18(2):194-211, 1979.

[30] M. R. Garey and D. S. Johnson. Computers and Intractability. A Guide to Theory of NP-Completeness. W.H.Freeman and Company., 1979.

[31] E. Gettier. Is justified true belief knowledge? Analysis, 23:121-123, 1963.

[32] R. Girle. Modal Logics and Philosophy. Acumen Publishing., 2000.

[33] A. Haddadi. Communication and cooperation in agent systems: A pragmatic theory. Lecture Notes in Artificial Intelligence, 1056:1-50, 1996.

[34] J. Y. Halpern and M. Y. Vardi. The complexity of reasoning about knowledge and time i: Lower bounds. J. of Computer and System Sciences, 38(1):195-237, 1989.

[35] J. Y. Halpern and Moses Y. Knowledge and commom knowledge in a distributed environment. Journal of ACM, 37(3):549-587, 1990.

[36] J. Y. Halpern and Moses Y. A guide to completeness and complexity for modal logics of knowledge and belief. Artificial Intelligence, 54:319-379, 1992. 
[37] A. G. Hamilton. Logic for Mathematicians. Cambridge, 1997.

[38] L. Hegenberg. Significado and Conhecimento. E.P.U. - Edusp, 1975.

[39] M. Henessy. Axiomatizing finite delay operators. Acta Informatica., $21: 61-168,1984$.

[40] M. Henessy and R. Milner. Algebraic laws for non-determinism and concurrency. Journal of Association for Computing Machinery., 32:137$161,1985$.

[41] J. Hintikka. Model for Modalities. D. Reidel Publishing Company, 1969.

[42] J. Hintikka. Is alethic modal logic possible? Acta Philosophica Fennica, $35,1982$.

[43] J. Hintikka. Reasoning about knowledge in philosophy: The paradigm of epistemic logic. In Theoretical Aspects of Reasoning About Knowledge. Proceedings of the 1986 Conference - Edited by Joseph $Y$. Halpern, 1986.

[44] J. Hintikka. The Principles of Mathematics Revisited. Cambridge University Press, 1996.

[45] J. E. Hopcroft and J. D. Ullman. Introduction to Automata Theory Languages and Computation, pages 146-176, 285-319. Addison-Wesley Publishing Co., 1979. Cap.: 7 e 12.

[46] G. Hunter. Metalogic: An Introduction to Metatheory of Standard First Order Logic. University of California Press, 1996.

[47] R. L. Kirkham. Theories of Truth. MIT Press, 1977.

[48] R. E. Ladner. The computational complexity of provability in systems of modal propositional logic. SIAM J. Comput., 6(3):467-480, 1977.

[49] L. Lamport. Time, clocks and the ordering of events in a distributed system. Communications of the ACM., 21(7):558-565, 1978.

[50] K. Lehrer. Knowledge, truht and evidence. Analysis, 25:168-175, 1965.

[51] W. Lenzen. Recent work in epistemic logic. Acta Philosophica Fennica, 30:1-98, 1978.

[52] H. Levesque. All i know: A study in autoepistemic logic. Artificial Intelligence, 42(3):263-309, 1990. 
[53] H. Levesque and J. des Rivières. The consistency of syntatical treatments of knowledge. In Theoretical Aspects of Reasoning About Knowledge. Proceedings of the 1986 Conference - Edited by Joseph $Y$. Halpern, 1986.

[54] B. Marchal. Modal logic - a brief tutorial. In Non-Standard Logics for Automated Reasoning, pages 15-22. Academic Press, 1988.

[55] F. Massacci. Tableaux methods for formal verification of multi-agent distributed systems. Journal of Logic Computat., 8(3):373-400, 1998.

[56] E. Mendelson. Introduction to Mathematical Logic. Van Nostrand Reinhold Company, 1964.

[57] S. Micali. Knowledge and efficient computation. In Theoretical Aspects of Reasoning About Knowledge. Proceedings of the 1986 Conference Edited by Joseph Y. Halpern, 1986.

[58] P. Milgrom. An axiomatic characterization of commom knowledge. Econometrica, 49(1), 1981.

[59] R. Milner. A modal characteriztion of observable machine-behaviour. Lecture Notes in Computer Science., 112:25-34, 1981.

[60] G. Mints. Short Introduction to Modal Logic. CSLI Publications, 1992.

[61] R. Montague. Syntactical treatments of modality with corollaries on reflection principle and finite axiomatizability. Acta Fennica., 16(2):153$167,1963$.

[62] G. Morril. Grammar and logic. Theoria, 62(Parte-3):260--293, 1996.

[63] J. Nolt and D. Rohatyn. Ló gica. McGraw-Hill, 1991.

[64] P. Panangaden and K. Taylor. Concurrent commom knowledge: defining agreement for asynchronous systems. Distributed Computing, (6):73-93, 1992.

[65] D. Park. Concurrency and automata on infinite sequences. Lecture Notes in Computer Science, 104, 1981.

[66] S. Popkorn. First Steps in Modal Logic. Cambridge University Press, 1994.

[67] V. R. Pratt. Semantical considerations on floyd-hoare logic. In Proc. 17th IEEE Symp. Foundations of Computer Science,, pages 109-121, 1976. 
[68] V. R. Pratt. Model of program logics. In Proc. 20th IEEE Symp. Foundations of Computer Science, pages 115-122, 1979.

[69] V. R. Pratt. Application of modal logic to programming. Studia Logica., 34:357-374, 1980.

[70] A. Ranta. Constructing possibles worlds. Theoria, 57(77-100):260-293, 1991.

[71] M. de Rijke. A system of dynamic modal logic. Technical report, University of Cambridge, 1992.

[72] W. J. Savitch. Relations between nondeterministic and deterministic tape complexities. J. Comput. and System Science, 4(2):177-192, 1970.

[73] K. Segerberg. Two-dimensional modal logics. Journal of Philosophical Logic, 2:77-96, 1973.

[74] R. M. Smullyan. First-Order Logic. Dover Publications, Inc., 1968.

[75] L. J. Stockmeyer. Classifying the computational complexity of problems. The Journal of Symbolic Logic., 52(1):1-43, 1987.

[76] L. J. Stockmeyer and A. R. Meyer. Word problems requiring exponential time: Preliminary report. In Proc. 5th Ann. ACM Symp. on Theory of Computing, pages 1-9, 1973.

[77] G. Sundholm. System of deduction. In D. Gabbay and F. Guenthner, editors, Handbook of Philosophical Logic. Vol. I: Elements of Classical Logic, pages 133-188. D. Reidel Publishing Co., Dordrecht, 1983. Cap.: I.2.

[78] A. Tarski. The semantic conception of truth and the foundations of semantics. Philosophy and Phenomenological Research, 4, 1944.

[79] S. K. Thomason. Noncompactness in propositional modal logic. Journal of Symbolic Logic, 37:714-720, 1972.

[80] S. K. Thomason. An incompleteness theorem in modal logic. Theoria, 40:30-34, 1974.

[81] A. S. Troelstra and H. Schwichtenberg. Basic Proof Theory, volume 43 of Cambridge Tracts in Theoretical Computer Science, pages 226-233. Cambridge University Press, 1996. Cap.: 9.1 e 9.2. 
[82] V. V. Tselishchev. Some philosophical problems of Hintikka's possible worlds semantics. In Logic, Foundations of Mathematics, and Computability Theory - Edited by R. E. Butts and J. Hintikka, volume 9, pages 391-403, 1977.

[83] J. van Benthem. Modal Logic and Classical Logic. Monographs in Philosofical Logic and Formal Linguistics. Bibliopolis, 1983.

[84] J. van Benthem. Correspondence theory. In D. Gabbay and Guenthner, F., editors, Handbook of Philosophical Logic. Vol. II: Extensions of Classic Logic, pages 167-247. D. Reidel Publishing Co., Dordrecht, 1984.

[85] J. van Benthem. A Manual of Intensional Logic. Lecture Notes. Center of the Study of Language and Information, 1988.

[86] A. Visser. Semantics and liar paradox. In D. Gabbay and Guenthner, F, editors, Handbook of Philosophical Logic. Vol. IV: Topics in the Philosophy of Language., pages 617-706. D. Reidel Publishing Co., Dordrecht, 1989.

[87] G. H. von Wright. An Essay in Modal Logic. Studies in Logic and the Foundations of Mathematics. North-Holland, 1951.

[88] M. Wooldridge and N. R. Jennings. Intelligent agents: Theory and pratice. Knowledge Engineering Review., 10(2):115-152, 1995.

[89] E. N. Zalta. A philosophical conception of propositional modal logic. Philosophical Topics, 21(2):263-281, 1993.

[90] E. N. Zalta. Basic Concepts in Modal Logic. CSLI - Stanford University, 1995. 


\section{Índice Remissivo}

\begin{tabular}{|c|c|}
\hline$B, 30$ & $\mathrm{KD} 45_{n}^{C}, 52$ \\
\hline 5,30 & $\mathrm{~K}_{n}, 13$ \\
\hline $\mathrm{CP}, 7$ & $\mathrm{~K}_{n}(\Phi), 14$ \\
\hline $\mathrm{CP}(\Phi), 7$ & $\mathrm{~K}_{n}^{C}, 52$ \\
\hline$C_{G}, 48$ & $K_{n}^{D}, 58$ \\
\hline$E_{G}, 48$ & $\stackrel{*}{M}, 61$ \\
\hline$\square$, ii & $\mathcal{M}_{n}, 21$ \\
\hline$\Delta, 14$ & $\mathcal{M}_{n}^{\text {elt }}, 35$ \\
\hline$\diamond$, ii & $\mathcal{M}_{n}^{r s t}, 35$ \\
\hline $\begin{array}{l}\mathcal{F}_{n}, 41 \\
\text { Telt }\end{array}$ & $\mathcal{M}_{n}^{r t}, 35$ \\
\hline $\mathcal{F}_{n}^{\text {elt }}, 41$ & $\mathcal{M}_{n}^{r}, 35$ \\
\hline $\begin{array}{l}\mathcal{F}_{n}^{r s t}, 41 \\
\mathcal{F}^{r t}\end{array}$ & $\Phi, 7$ \\
\hline $\begin{array}{l}\mathcal{F}_{n}^{r t}, 41 \\
\mathcal{F}^{r}, 41\end{array}$ & PDL, ii \\
\hline $\begin{array}{l}\mathcal{F}_{n}^{r}, 41 \\
G \text {-atingivel, } 49\end{array}$ & 4,29 \\
\hline$G$-atingivel, 49 & $\Sigma_{\text {con }}, 16$ \\
\hline $\begin{array}{l}\Gamma_{\mathrm{AX}} \\
\quad \text { consistente, } 60\end{array}$ & $\Sigma_{\operatorname{maxc}}, 16$ \\
\hline estrutura de Kripke, 61 & $\mathrm{~S}, 8$ \\
\hline pseudo-estrutura, 61 & $S H, 9$ \\
\hline pseudo-satisfativel, 61 & S4, 29 \\
\hline satisfatível, 61 & $S 4_{n}, 29$ \\
\hline $\mathrm{K}, 13$ & $S 4_{n}, 29$ \\
\hline$K, 14$ & $\mathrm{~S} 4_{n}^{C}, 52$ \\
\hline $\mathcal{K}_{D_{G}}, 61$ & $S 4_{n}^{D}, 58$ \\
\hline$K_{i}, 13,20$ & S5, 29 \\
\hline $\mathcal{K}_{i}$-Sucessor, 102 & $\mathrm{~S} 5_{n}, 29$ \\
\hline $\mathrm{K}_{n}$-dedutível, 16 & $\mathrm{~S} 5_{n}, 30$ \\
\hline $\mathrm{KD} 45,29$ & $S 5_{n}^{c}, 52$ \\
\hline $\mathrm{KD} 45_{n}, 29$ & $\mathrm{~S} 5_{n}^{D}, 58$ \\
\hline
\end{tabular}




$$
\begin{aligned}
& \mathbb{T}, 93 \\
& \mathrm{~T}, 64 \\
& T, 29 \\
& \mathrm{~T}, 29 \\
& \mathrm{~T}_{n}, 29 \\
& \mathrm{~T}_{n}, 29 \\
& \mathrm{~T}_{n}^{C}, 52 \\
& \mathrm{~T}_{n}^{D}, 58 \\
& \models^{D}, 10,20 \\
& \models, 61 \\
& \neg, 7 \\
& \rightarrow, 7 \\
& \tau, 8 \\
& \vdash, 8,10 \\
& \vdash_{\mathrm{CP}}, 8 \\
& \vdash_{\kappa_{n}} \varphi, 16 \\
& \vee, 8 \\
& \wedge, 8
\end{aligned}
$$

Agente, 1

Agentes epistêmicos, 1

Alternating, 115

Aresta, 49

Aristóteles, 2

Árvore, 64 de prova, 9

Assíncrono, 48

Aumann, R., 48

Avron, A., 10

Axiomática método, 1

Axioma, 8

Conhecimento Mútuo, 52

Ponto-Fixo, 52

Axiomas

de $\mathrm{K}_{n}, 14$

esquema de, 7

Bacharach, M., 48

Bicchieri, C., 48
Bit, 109

Bivaloração, 18

Blackburn, P., iii

Bom senso, 47

Boolos, G., 2

Bostok, D., 7

Brower, L. E. J, 29

Bull, R., iii

Cadeia

vide string, 109

Caminho, 49 não-redundante, 66

primitivo, 65,96

redução, 65

reduzido, 65

Carnap, R., 3

Carpenter, B., i

Chellas, B., iii

Chiara, M. L. D., i

Church, A., 3, 74

Classe

$\mathcal{C}$-completa, 80

$\mathcal{C}$-difícil, 80

$\mathcal{C}$-log espaço completo, 80

de estruturas, 21

Composicionalidade, 10

Computável log-espaço, 79

ver Decidível, 79

Computação, 78

determinística, 78

espaço de, 79

não-determinística, 78

tempo de, 78

Conhece, 1

Conhecimento

comum, 47

comum concorrente, 48

distribuído, 58

mútuo, 47 
Conjunto

completamente expandido, 97

evidentemente inconsistente, 97

Conseqüência

lógica, 10

semântica, 10

sintática, 10

Consistente

conjunto, 6,16

fórmula, 16

Contexto

referencialmente transparente, 3

referencialmente opaco, 3

Cresswel, M., 13

da Costa, N. C., iii

Decidível

em espaço não-determinístico, 79

em tempo não-determinístico, 79

ver Computável, 79

Decisāo, 39

Dedução, 15

meta-teorema, 9

del Cerro, L. F., iii

Depth-First-Search, 103

Desembaraçar, 64

Dunn, J. M., iii

Emerson, E. A., i

Estóicos, 2

Estado, 102

de programa, ii

Estrutura

baseada num frame, 20

de Kripke, 20

equivalente, 39

pseudo, 61 tipo árvore, 67

Fórmula, 7

atômica, 7

bem-formada, 81

booleana quantificada, 81

contraditória, 30

tamanho de uma, 7

testemunha, 97

Fórmulas

modais irredutíveis, 18

Fagin, R., i

Fecho, 39

reflexivo, 39

Fine, K., 4

Fischer, M. J., i

Floresta, 64

Frame, 20

Frege, G., 3

Função

de valoração, ii

rotuladora, 94

Gödel, K., 30, 74

Garey, M. R., iii

Gettier, E., iii, 7

Grafos, 49

Gramática

de Montague, 2

Halpern, J. Y., i

Hamilton, A. G., 7

Harsany, J., 48

Hegenberg, L, 1

Henessy, M., i

Hintikka, J., iii, 3

Hugues, G., 13

Hume, D., 48

Ideais

elementos, 14

Inconsistente 


\begin{tabular}{|c|c|}
\hline conjunto, 16 & Mendelson, E., 7 \\
\hline Interpretação & Meyer, A. R., iii \\
\hline Modal, 18 & Micali, S., iii \\
\hline modal, 18 & Milgrom, P., iii \\
\hline Introspecção & Milner, R., i \\
\hline negativa, 30 & Mints, G., ii \\
\hline Intuicionismo, 29 & Modalidade \\
\hline $\begin{array}{l}\text { Kanger, S., } 3 \\
\text { Kirkham, R. L., iii } \\
\text { Kripke, S., } 3\end{array}$ & $\begin{array}{l}\text { gramatical, ii } \\
\text { Modelo, } 36 \\
\text { frame como, } 41 \\
\text { Molde, } 20\end{array}$ \\
\hline $\begin{array}{l}\text { Lógica } \\
\text { da Prova, } 2 \\
\text { das fórmulas booleanas quan- } \\
\quad \text { tificadas, } 81 \\
\text { quântica, i, } 30 \\
\text { temporal, i }\end{array}$ & $\begin{array}{l}\text { Montague, R., } 2 \\
\text { Morril, G., i } \\
\text { Moses, Y., i } \\
\text { Mundo } \\
\quad \text { atingível, } 49 \\
\quad \text { possível, } 18\end{array}$ \\
\hline Ladner, R. E., iii & Nó \\
\hline Lehrer, K., iii & i-filho, 65 \\
\hline Leibniz, G. W., 3 & filho, 64 \\
\hline Lenzen, W., iii & interno, 102 \\
\hline Levesque, H., iii & pai, 64 \\
\hline Lewis, C. I., 2 & Noçoes \\
\hline Lewis, D., 48 & extensionais, 3 \\
\hline $\begin{array}{l}\text { Lindenbaun } \\
\qquad \text { lema de, } 16\end{array}$ & intensionais, 3 \\
\hline Littlewood, J. E., 48 & $\mathbf{u}$ \\
\hline Lukasevicz, J., 3 & Organon, 2 \\
\hline MacColl, H., 2 & park, D., i \\
\hline Mackinsey, J. C. C., 3 & Período \\
\hline Marcus, R. B., 3 & algébrico, 3 \\
\hline Massaci, F., i & da semântica via modelos, 4 \\
\hline Matriz & sintático, 3 \\
\hline $\mathrm{da} q b f, 81$ & Ponto Fixo, 51 \\
\hline Maximal & ponto no tempo, ii \\
\hline consistente, 16 & Pratt, V. R., i \\
\hline Maxinal & Princípio de Indução Finita, 52 \\
\hline conjunto, 6 & Prior, A., 3 \\
\hline McCarthy, J., 47 & Programas \\
\hline Megáricos, 2 & primitivos, 115 \\
\hline
\end{tabular}


Proposição, 1

Propriedades epistêmicas, 29

Prova

a partir de premissas, 15

Provabilidade, 28

Quantificador universal restrito, 14

Quine, W. von O., 3

Ranta, A, 6

Redutível

log-espaço, 79

em tempo polinomial, 79

Referencial

opaco, 3

Referencialmente

opaco

Vide Contexto, 3

transparente

Vide Contexto, 3

Regra

de generalização modal, 11,14

de generalização universal, 11

de Indução, 52

de inferência, 7,10

Regras

substituição, 3

Relação

de acessibilidade, 20

de Satisfatibilidade, 20

euclideana, 32

serial, 32

Rijke, M. de, ii

Síncrono, 48

Sabe, 1

Satisfatível, 21

pseudo, 61

Savitch, 80

Scheling, T., 48

Schiffer, S., 48
Segerberg, K., iii

Semântica

dos mundos possíveis, 3

Silogismo hipotético, 9

Simular

uma estrutura, 64

Stockmeyer, L. J., iii

String, 109

Substituição, 8 em $\mathrm{K}_{n}, 14$

Tableau proposicional, 93

Tableaux completamente expandido, 96 pré-, 101

Tamanho de uma estrutura, 42

Tarski, A, 3

Teorema

de $\mathrm{CP}, 8$

em $\mathrm{K}_{n}, 14$

Teoria

da correspondência, 4, 34

Thomason, S. K., 4

Tree-like, 67

Tselischev, V. V., iii

Turing, A., 74

Válida, 21

Vértice, 49

Validade

de uma $q b f, 81$

van Benthem, J., i

Vardi, M. Y., i

Verdade, 4

teoria da correspondência, 4

valor, 3

Visser, A., 5

von Wright, G.H., iii

Zalta, E. N., iii 